\title{
Physical Verification of the Melt Pool in Laser-Bed Fusion
}

\author{
Giordimaina, Andre
}

How to cite:

Giordimaina, Andre (2017) Physical Verification of the Melt Pool in Laser-Bed Fusion. thesis, Swansea University. http://cronfa.swan.ac.uk/Record/cronfa49707

Use policy:

This item is brought to you by Swansea University. Any person downloading material is agreeing to abide by the terms of the repository licence: copies of full text items may be used or reproduced in any format or medium, without prior permission for personal research or study, educational or non-commercial purposes only. The copyright for any work remains with the original author unless otherwise specified. The full-text must not be sold in any format or medium without the formal permission of the copyright holder. Permission for multiple reproductions should be obtained from the original author.

Authors are personally responsible for adhering to copyright and publisher restrictions when uploading content to the repository.

Please link to the metadata record in the Swansea University repository, Cronfa (link given in the citation reference above.)

http://www.swansea.ac.uk/library/researchsupport/ris-support/ 


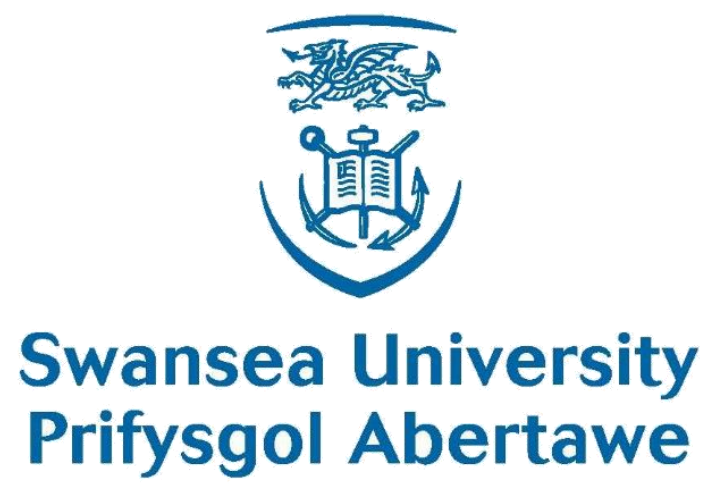

\title{
Physical Verification of the Melt Pool in Laser Powder-Bed Fusion
}

\author{
Andre Giordimaina
}

Submitted to Swansea University in fulfilment of the requirements for the Degree of Engineering Doctorate

Swansea University

Materials Research Centre 


\section{Acknowledgements}

The research presented here was funded by my industrial sponsor, Renishaw plc, and MATTER, funded by the EPSRC through Swansea University.

l'd like to thank both my parents for showing me steadfast dedication, as well as my academic supervisors, Dr Nick Lavery and Professor Steve Brown, for showing infinite patience and astute guidance. l'd like to give them all special thanks for helping me get this far. I want to say thanks as well to all my friends and colleagues who've helped me out and been there for me. 


\section{Declaration}

This work has not previously been accepted in substance for any degree and is not being concurrently submitted in candidature for any degree.

Signed (candidate)

Date

\section{STATEMENT 1}

This thesis is the result of my own investigations, except where otherwise stated. Where correction services have been used, the extent and nature of the correction is clearly marked in a footnote(s).

Other sources are acknowledged by footnotes giving explicit references. A bibliography is appended.

Signed (candidate)

Date

\section{STATEMENT 2}

I hereby give consent for my thesis, if accepted, to be available for photocopying and for inter-library loan, and for the title and summary to be made available to outside organisations.

Signed (candidate)

Date 


\section{Table of Contents}

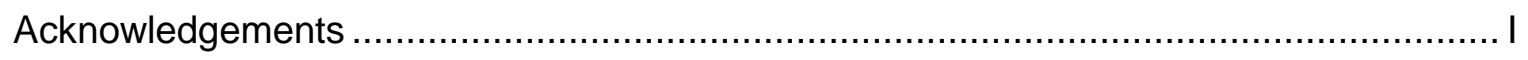

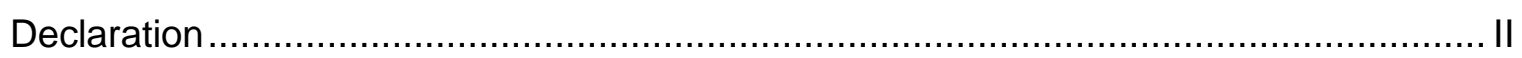

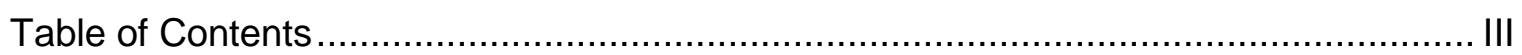

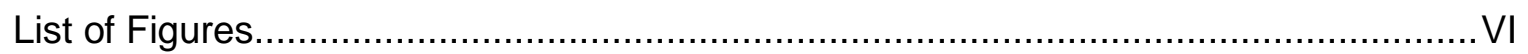

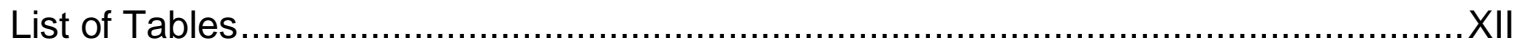

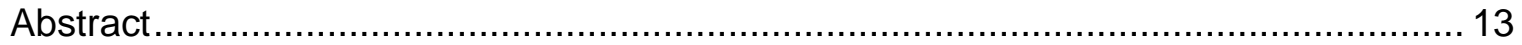

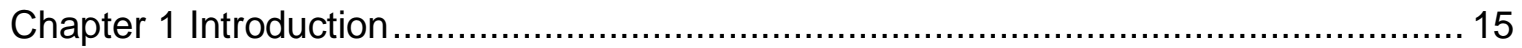

1.1 An Introduction to Additive Manufacturing ................................................ 15

1.2 Limitations and Issues concerning Powder-Bed Fusion ...............................17

1.3 State-of-the-Art in Generating Process Maps ........................................... 18

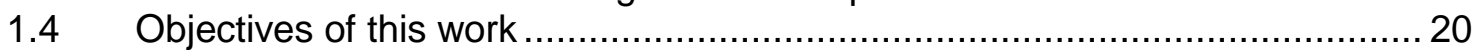

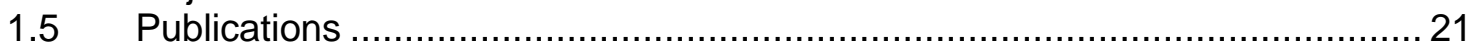

Chapter 2 Overview of Additive Manufacturing Systems ………................................. 22

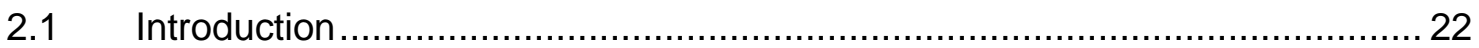

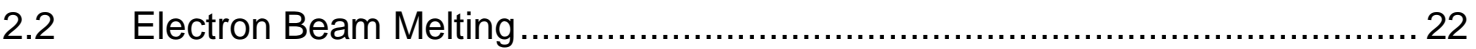

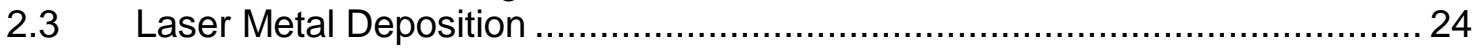

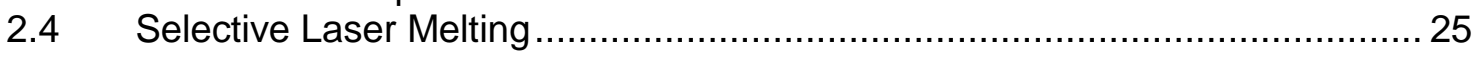

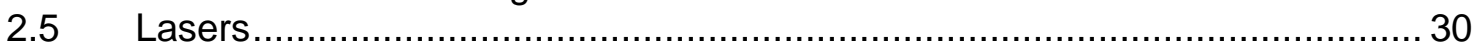

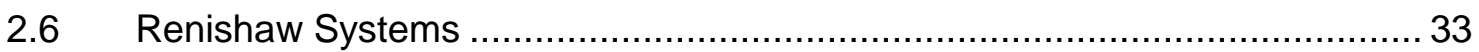

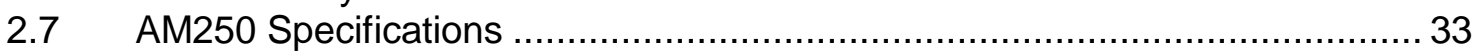

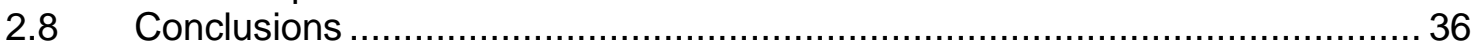

Chapter 3 Thermo-Mechanics of melt pool formation ................................................ 37

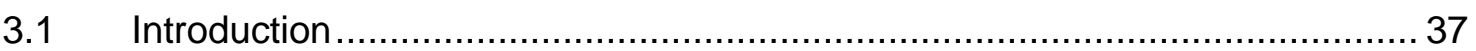

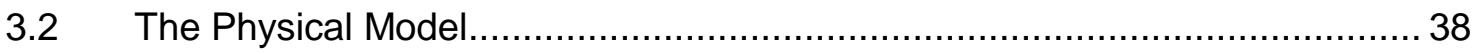

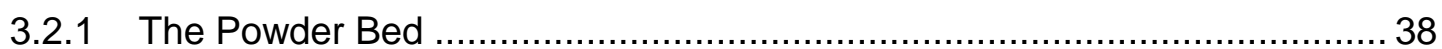

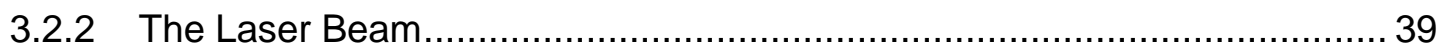

3.2.3 Melting of the Powder Bed ……….................................................... 40

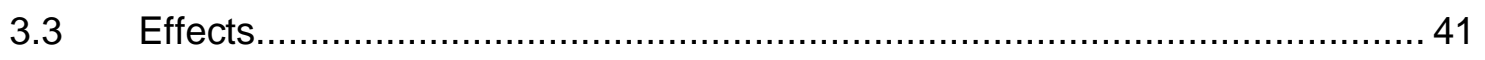

3.3.1 Wetting and Balling ............................................................................ 41

3.3.2 Plateau-Rayleigh Instability ........................................................... 43

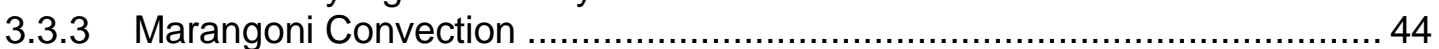

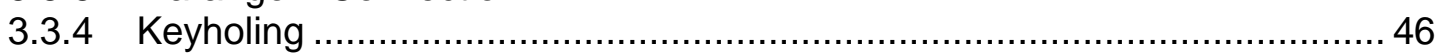

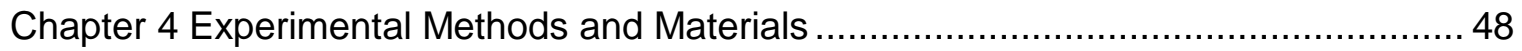

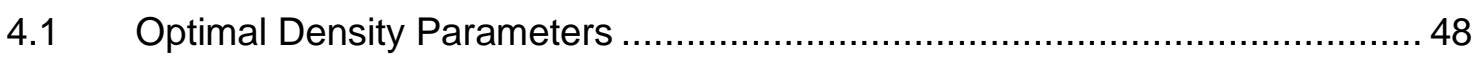

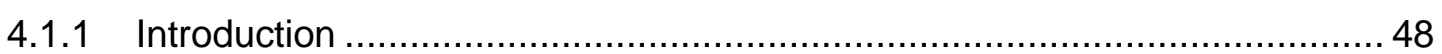

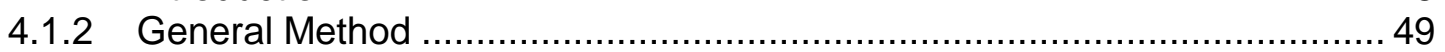


4.1.3 Stainless Steel 316L Powder (Experiments A and B) ............................50 50

4.1.4 Stainless Steel 316L Powder (Experiments $C$ and D) .............................51

4.1.5 Titanium Ti6Al4V Powder (Experiment E) ............................................. 53

4.2 Experiment A - Direct Base Plate Method ................................................. 54

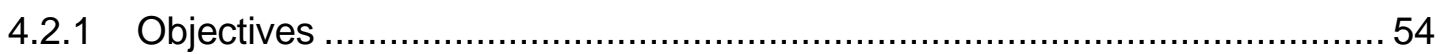

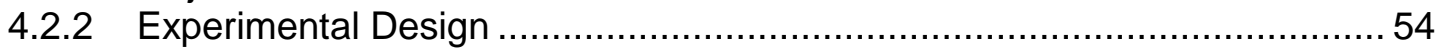

4.3 Experiment B - Single-Lines on Recessed Plates Method........................... 56

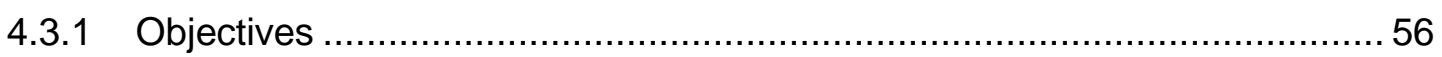

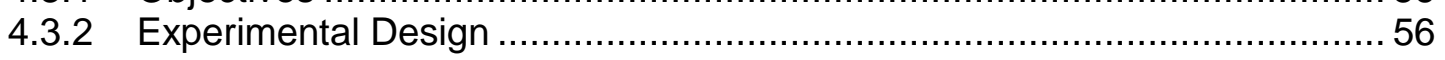

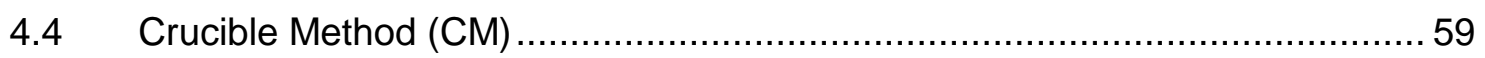

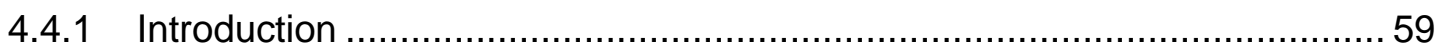

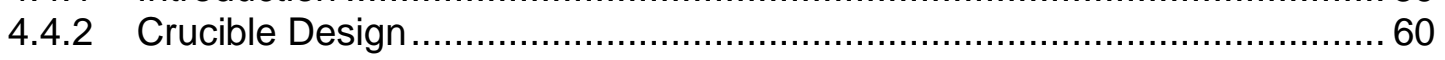

4.4.3 Experiment C - Verification of Single-Track Crucible Methodology ...............62 62

4.4.4 Experiment D - Single-Tracks on Crucible Substrates ................................6 64

4.4.5 Experiment E - Crucible Single-Track experiments using Ti-6Al-4V.............. 65

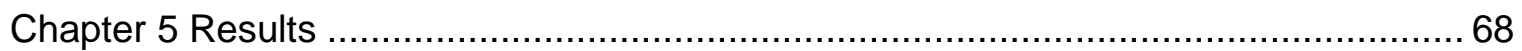

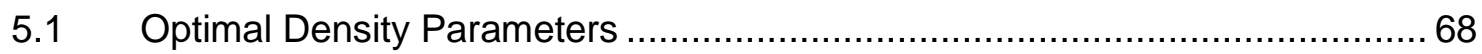

5.1.1 Stainless Steel 316L Powder (Experiments A and B) ..............................6 68

5.1.2 Stainless Steel 316L Powder (Experiments $C$ and D) ............................69 69

5.1.3 Titanium Ti6Al4V Powder (Experiment E) ................................................ 70

5.2 Experiment A- Direct Base Plate Method ................................................... 71

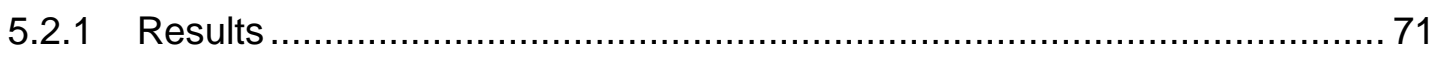

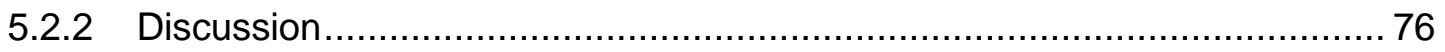

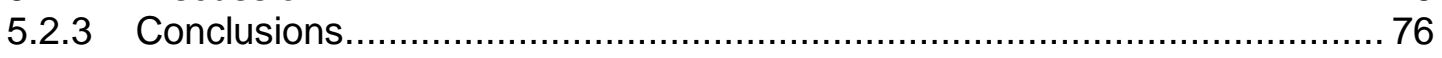

5.3 Experiment B - Single-Lines on Recessed Plates Method........................... 77

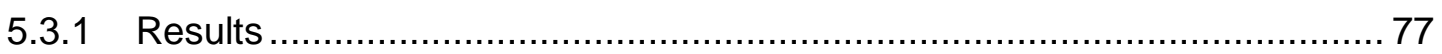

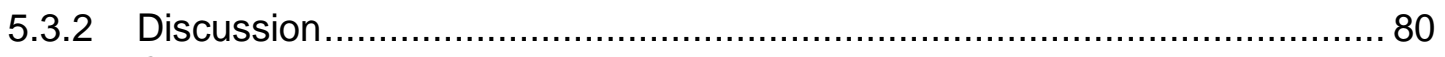

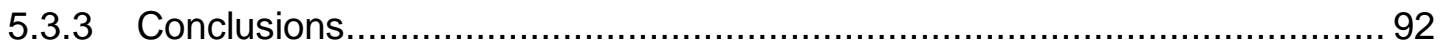

5.4 Experiment C - Verification of Single-Track Crucible Methodology ................94

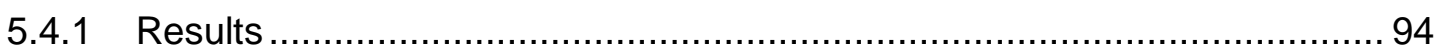

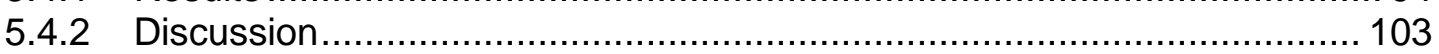

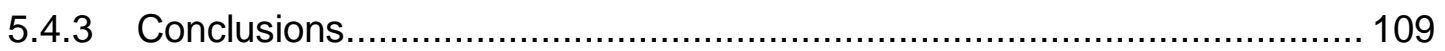

5.5 Experiment D - Single-Tracks on Crucible Substrates............................ 111

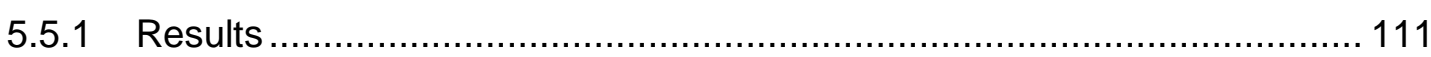

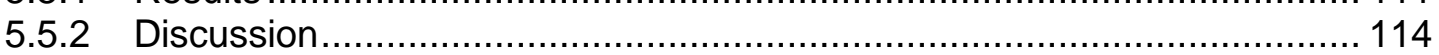

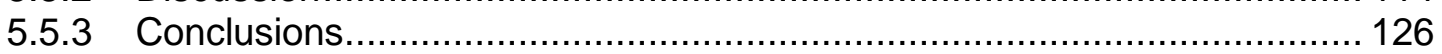

5.6 Experiment E - Crucible Single-Track experiments using Ti-6Al-4V ........... 128

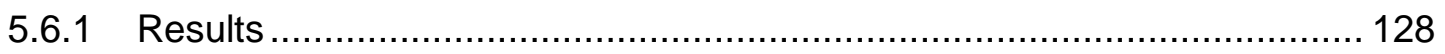

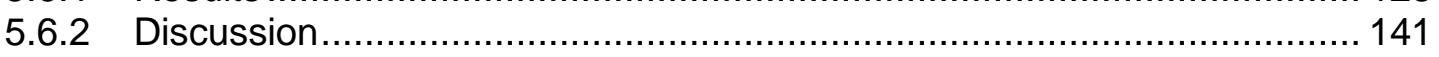

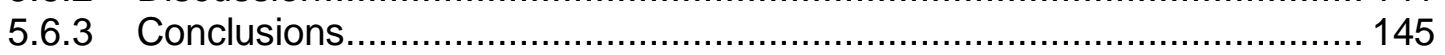

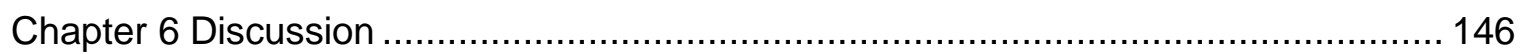

Chapter 7 Conclusions and Further Work..................................................... 152

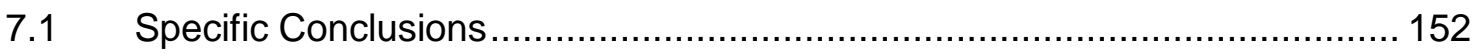


7.2 General Conclusions.

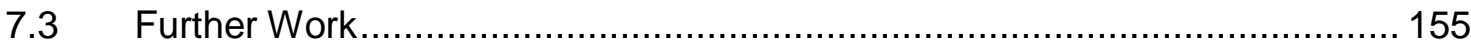

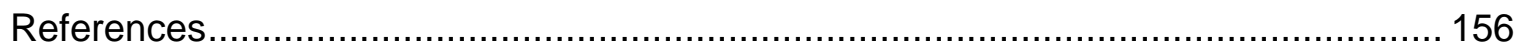

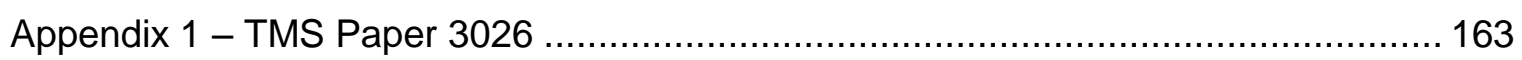

Appendix 2 - Metallographic Preparation for Experiment A .................................... 170

Appendix 3 - Metallographic Preparation for Experiment C .................................... 171

Appendix 4 - Beraha II Etchant Preperation............................................................... 172

Appendix 5 - Metallographic Preparation for Experiment E ................................... 174 


\section{List of Figures}

Figure 1. Laser Metal Deposition Apparatus. .................................................................... 25

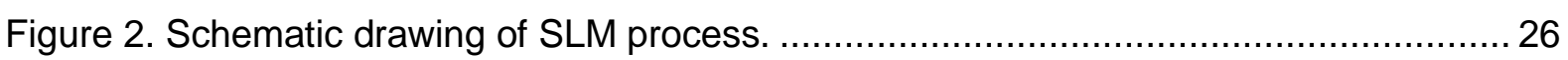

Figure 3. Absorption of laser output at different wavelengths .................................... 27

Figure 4. Laser focus positions with respect to the powder bed. ....................................... 29

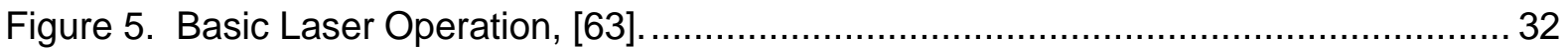

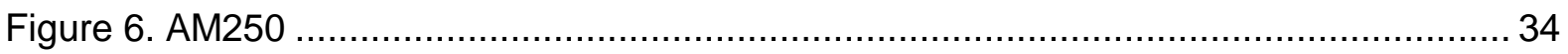

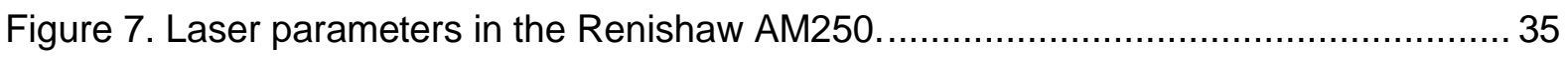

Figure 8. Physical phenomena at play in the powder-bed fusion system.......................... 38

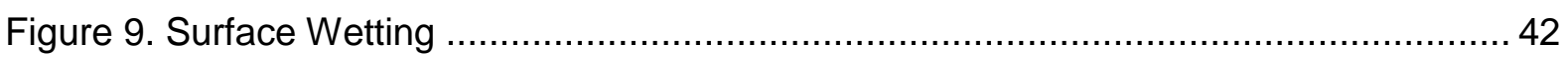

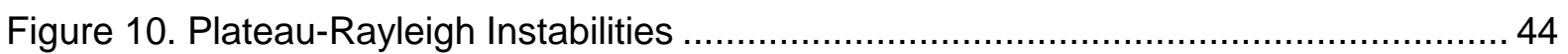

Figure 11. Right) Layout of the machine parameter array on the build plate, Left) as-built sample labels 51

Figure 12. Left) Density cubes, as they appeared in the assembly diagram, Right) the as-

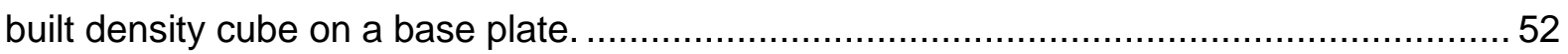

Figure 13. Experiment $A(S S 316 L)$ - Experimental Design .......................................... 55

Figure 14. Experiment B (SS316L) - Experimental design. ....................................... 58

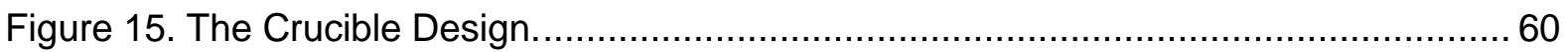

Figure 16. Left) CAD drawing of crucibles used during an experiment, Right) Three single

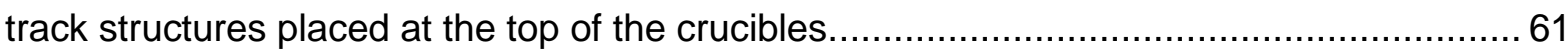

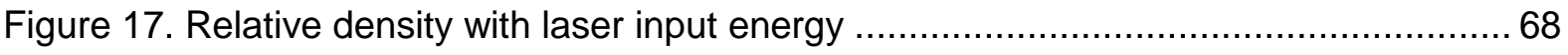

Figure 18. Relative density with input energy measured in DOE experiment for Chapter 5, (SS316L)

Figure 19. Relative density with input energy measured in DOE experiment for Chapter 6, (Ti-6Al-4V)

Figure 20. Experiment A (SS316L) - Cross-sectional and topographical results, Sample 1. 72 
Figure 21. Experiment A (SS316L) - Cross-sectional and topographical results, Sample 2. 72 Figure 22. Experiment A (SS316L) - Cross-sectional and topographical results, Sample 3. 73 Figure 23. Experiment A (SS316L) - Cross-sectional and topographical results, Sample 4. 73 Figure 24. Experiment A (SS316L) - Cross-sectional and topographical results, Sample 5. 74 Figure 25. Experiment A (SS316L) - Cross-sectional and topographical results, Sample 6. 74 Figure 26. Experiment $A$ (SS316L) - Changes in the track dimensions and gap sizes.........75 Figure 27. Experiment B (SS316L) - Topographical process map at 50 $\mu$ m layer depth. The red dot shows the shows the parameters used at the Renishaw recommended operating conditions 78

Figure 28. Experiment B (SS316L) - Cross-sectional process map at 50 4 m layer depth.... 79 Figure 29. Experiment B (SS316L) - The five types of topographical tracks which formed during experiment.

Figure 30. Experiment B (SS316L) - The three types of cross-sectional tracks formed during experiment.

Figure 31. Experiment B (SS316L) - Variation of the melt pool stability with scan speed. ... 82 Figure 32. Experiment B (SS316L) - Track width at the $75-125 \mathrm{~W}$ range compared to results from Bertoli et al 85

Figure 33. Experiment B (SS316L) - Track width at the 150-200W range compared to results from Bertoli et al 85

Figure 34. Experiment B (SS316L) - Track depth at the 75-125W range compared to results from Bertoli et al 86

Figure 35. Experiment B (SS316L) - Track depth at the 150-200W range compared to results from Bertoli et al 86 Figure 36. Experiment B (SS316L) - Comparison of measured and predicted melt pool depths at the $75-125 \mathrm{~W}$ range according to equation from Gladush and Smurov 89 Figure 37. Experiment B (SS316L) - Comparison of measured and predicted track depths at the 150-200W range according to equation from Gladush and Smurov 89 
Figure 38. Experiment B (SS316L) - Topographical images of tracks built using parameters

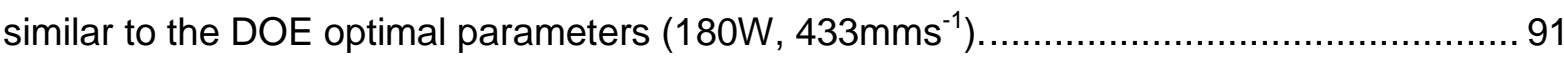

Figure 39. Experiment B (SS316L) - Contours of build ratios of single tracks.................... 91

Figure 40. Experiment B (SS316L) - Contours of depth to width ratio of single tracks........ 92

Figure 41. Experiment C (SS316L) - Topographical process map at 50 um layer depth...... 95

Figure 42. Experiment C (SS316L) - Cross-sectional process map at $50 \mu \mathrm{m}$ layer depth.... 96

Figure 43. Experiment C (SS316L) - Topographical process map at $100 \mu \mathrm{m}$ layer depth.... 97

Figure 44. Experiment C (SS316L) - Cross-sectional process map at $100 \mu \mathrm{m}$ layer depth.. 98

Figure 45. Experiment C (SS316L) - Topographical process map at $150 \mu \mathrm{m}$ layer depth.... 99

Figure 46. Experiment C (SS316L) - Cross-sectional process map at 150 $\mathrm{mm}$ layer depth.100 Figure 47. Experiment C (SS316L) - Topographical process map at $200 \mu$ m layer depth.. 101 Figure 48. Experiment C (SS316L) - Cross-sectional process map at $200 \mu \mathrm{m}$ layer depth 102 Figure 49. Experiment C (SS316L) - The three types of tracks formed. 104

Figure 50. Experiment C (SS316L) - Left) Tracks build on the crucible substrate, with distinctive, flattened tracks appearing on the left-hand side. Right) Cross-sectional image taken using the same parameters. 104

Figure 51. Keyhole formation and fluid flow in the melt pool, taken from Stanciu et al. [113]

Figure 52. Experiment C (SS316L) - Contours of depth-to-width ratios at the different layer depths. 109

Figure 53. Experiment D (SS316L) - Topographical process map................................ 112

Figure 54. Experiment D (SS316L) - Cross-sectional process map. 113 Figure 55. Experiment D (SS316L) - Track widths compared with Experiment B (SS316L) at the $75-125 \mathrm{~W}$ range 115 Figure 56. Experiment D (SS316L) - Track widths compared with Experiment B (SS316L) at the $150-200 \mathrm{~W}$ range. 115

Figure 57. Experiment D (SS316L) - The three types of tracks formed. 118

Figure 58. Experiment D (SS316L) - Keyhole porosity observed during experiment. 119 
Figure 59. Experiment D (SS316L) - Track depths compared with Experiment B (SS316L) at the $75-125 \mathrm{~W}$ range. 120

Figure 60. Experiment D (SS316L) - Track depths compared with Experiment B (SS316L) at the 150-200W range.

Figure 61. Experiment D (SS316L) - Comparison of measured and predicted penetration depths according to equation from Gladush and Smurov

Figure 62. Experiment D (SS316L) - Comparison of measured and predicted penetration depths according to equation from Gladush and Smurov 122

Figure 63. Experiment D (SS316L) - Left) Topographical image of track built using parameters similar to DOE optimal parameters, Right) cross-section taken at the same track.

Figure 64. Experiment D (SS316L) - Contours of line build percentage of single tracks. ... 124

Figure 65. Experiment D (SS316L) - Contours of depth-to-width ratio. 125

Figure 66. Experiment D (SS316L) - Cross section of a track, highlighting the type of grain formation. 126

Figure 67. Experiment E (Ti-6Al-4V) - Topographical process map at 50 $\mu$ m layer depth.. 128 Figure 68. Experiment E (Ti-6Al-4V) - Cross-sectional process map at 50 4 m layer depth.129 Figure 69. Left) Experiment E (Ti-6Al-4V) -Single Tracks, produced at 200W, 500mms ${ }^{-1} .130$ Figure 70. Experiment E (Ti-6Al-4V) - Left) Continuous tracks built at $200 \mathrm{~W}, 500 \mathrm{mms}^{-1}$, Right) continuous tracks and droplet formation, built at 200W, $750 \mathrm{mms}^{-1}$, crucible depth of $50 \mu \mathrm{m}$ 131

Figure 71.Experiment $\mathrm{E}(\mathrm{Ti}-6 \mathrm{Al}-4 \mathrm{~V})$ - Track cross-section, taken at $150 \mathrm{~W}, 1000 \mathrm{mms}^{-1}$.

Necking occurs between the melt bead and substrate. 132

Figure 72. Experiment E (Ti-6Al-4V) - Topographical process map at $100 \mu \mathrm{m}$ layer depth.133 Figure 73. Experiment E (Ti-6Al-4V) - Cross-sectional process map at $100 \mu \mathrm{m}$ layer depth. 
Figure 74. Experiment $\mathrm{E}(\mathrm{Ti}-6 \mathrm{Al}-4 \mathrm{~V})$ - Left) Continuous tracks built at $200 \mathrm{~W}, 500 \mathrm{mms}^{-1}$, Right) continuous tracks and droplet formation, built at 200W, $750 \mathrm{mms}^{-1}$, crucible depth of $100 \mu \mathrm{m}$ 134

Figure 75. Experiment $\mathrm{E}(\mathrm{Ti}-6 \mathrm{Al}-4 \mathrm{~V})$ - Left) Continuous tracks built at $100 \mathrm{~W}, 500 \mathrm{mms}^{-1}$, Right) continuous tracks and droplet formation, built at $100 \mathrm{~W}, 750 \mathrm{mms}^{-1}$, crucible depth of $100 \mu \mathrm{m}$ 135

Figure 76. Experiment E (Ti-6Al-4V) - Topographical process map at $150 \mu \mathrm{m}$ layer depth.136 Figure 77. Experiment E (Ti-6Al-4V) - Cross-sectional process map at $150 \mu \mathrm{m}$ layer depth.

Figure 78. Experiment E (Ti-6Al-4V) - Left) Continuous tracks built at 200W, $500 \mathrm{mms}^{-1}$, Right) continuous tracks and droplet formation, built at 200W, $750 \mathrm{mms}^{-1}$, crucible depth of $150 \mu \mathrm{m}$

Figure 79. Experiment E (Ti-6Al-4V) - Left) Continuous tracks built at $150 \mathrm{~W}, 500 \mathrm{mms}^{-1}$, Right) continuous tracks and droplet formation, built at $150 \mathrm{~W}, 750 \mathrm{mms}^{-1}$, crucible depth of $150 \mu \mathrm{m}$ 138

Figure 80. Experiment E (Ti-6Al-4V) - Topographical process map at $200 \mu \mathrm{m}$ layer depth.139 Figure 81. Experiment E (Ti-6Al-4V) - Cross-sectional process map at $200 \mu \mathrm{m}$ layer depth.

Figure 82. Experiment E (Ti-6Al-4V) - Left) Continuous tracks built at $200 \mathrm{~W}, 500 \mathrm{mms}^{-1}$, Right) continuous tracks and droplet formation, built at $200 \mathrm{~W}, 750 \mathrm{mms}^{-1}$, crucible depth of $200 \mu \mathrm{m}$ 140

Figure 83. Experiment E (Ti-6Al-4V) - Tracks formed at $200 \mathrm{~W}, 500 \mathrm{mms}^{-1}$, at increasing layer thicknesses. 142

Figure 84. Experiment E (Ti-6Al-4V) - Average Track Width at 200W. 143

Figure 85. Experiment E (Ti-6Al-4V) - Average Track Width at 150W. 144

Figure 86. Experiment E (Ti-6Al-4V) - Average Track Width at 100W. 144

Figure 87. Transition of the track geometry in Experiment B (SS316L). 147

Figure 88. The five types of tracks that formed during Experiment B (SS316L)..... 148 
Figure 89. Transition of the track geometry in Experiment D (SS316L)......................... 148

Figure 90. Transition of the track geometry in Experiment C (SS316L)......................... 149 


\section{List of Tables}

Table 1. Spot size calibration of the Renishaw AM250 used in this work............................ 34

Table 2. Typical laser parameters for the Renishaw AM250........................................ 36

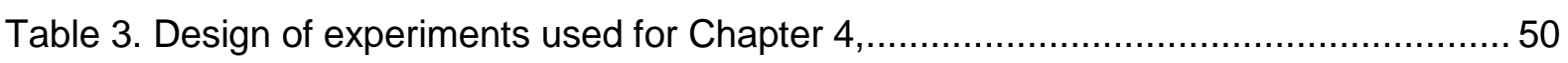

Table 4. Composition, in weight percentage, of the 316L powder used in the study ............50

Table 5. Laser parameters used for DOE for Chapter 5 ............................................. 52

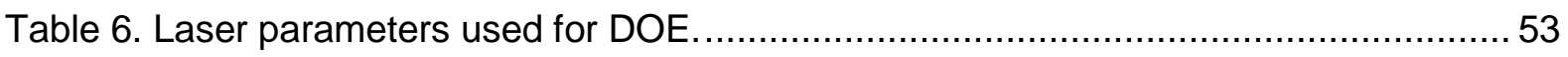

Table 7. Experiment A (SS316L) - Laser Parameters used.......................................... 54

Table 8. Experiment B (SS316L) - Processing parameters used.....................................57

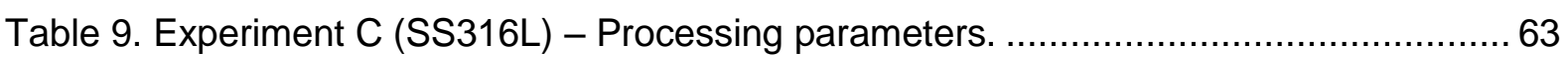

Table 10. Experiment D (SS316L) - Processing parameters used................................... 65

Table 11. Experiment E (Ti-6Al-4V) - Processing parameters used.....................................66

Table 12. Experiment A (SS316L) - Average Length, Width, Height and Gap Size taken from

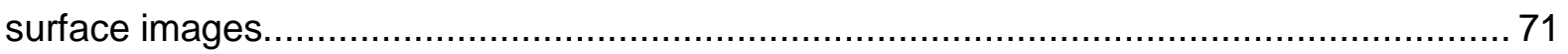

Table 13. Experiment B (SS316L) - Process Map with line build percentages, divided into

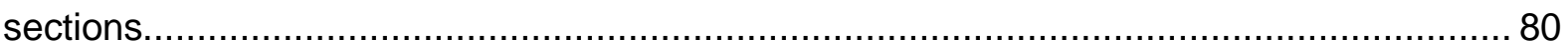

Table 14. Values used to calculate values for penetration depth equation from Gladush and

Smurov

Table 15. Experiment C (SS316L) - Process maps with line build percentages for each layer

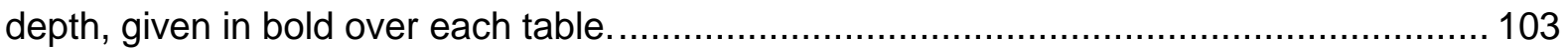

Table 16. Experiment D (SS316L) - Process map with line build percentages.................. 111 Table 17. Experiment E (Ti-6Al-4V) - Process map with line build percentages for each layer depth, given in bold over each table. 


\section{Abstract}

Laser Powder-Bed Fusion (LPBF) is an additive manufacturing process which fuses metal powder on a layer by layer basis to form complex three-dimensional components. As with other additive processes, LPBF is seeing a rapid evolution of machine design, scanning techniques, and materials development which has moved the process well beyond its origins in rapid prototyping to a process which can manufacture fit-for-purpose components. At the heart of the LPBF process lies the melt pool, and the way in which the laser properties, such as speed, power and beam diameter interact to form tracks fused to the substrate is integral to the way in which multiple tracks will fill the contours across each layer in the build sequence.

Controlling the as-solidified bead shape is important to ensure optimal mechanical properties. A widespread technique for measuring the effect of laser properties on the mechanical properties and track formation is process mapping. Single-layer or single-track process maps, which measure the behaviour of the melt according to laser properties on a single layer of powder, have been limited to a base plate of same composition, but with a different microstructure, typically resulting from a rolling process.

The work in this thesis describes the efforts to standardise a high-throughput method of creating process maps which measure the effects of these process parameters on, in a way which compliments and improves upon the usual technique of deposition of single line tracks directly onto a base plate. One result of this work is a new method where substrates are built using the LPBF process, on which single tracks are deposited with a controlled powder depth. This is done in such a way that the as-built tracks are representative of the process at regions away from the base plate, by building the substrate in-situ, before the forming of the tracks.

It was found that the crucible single track method could be used quite effectively to control the powder layer depth at which tracks were deposited on. The additional benefit granted by 
the crucible substrate was the ease at which high quality topographical and cross-sectional metallography could take place in order to quantify and investigate the effects of changing the parameters. For example, by using the crucible method, it was found that titanium alloy Ti-6Al-4V, at a maximum laser power of $200 \mathrm{~W}$, could form relatively stable track formations at $100 \mu \mathrm{m}$ layer thickness at a scan speed of $500 \mathrm{mms}^{-1}$. At lower power values, faster scan speeds or larger layer depths, tracks would not form successfully.

Another important outcome was that the crucible method predicted a much less severe transition between conductive and keyhole modes of melting than direct deposition of single tracks onto a baseplate, with shallower re-melting of lower layers. The crucible method also predicted a more forgiving transition between continuous lines and lines which had broken down due to poor wetting or insufficient temperatures. 


\section{Chapter 1 Introduction}

\subsection{An Introduction to Additive Manufacturing}

Additive manufacturing (AM) is an umbrella term to describe a wide number of manufacturing techniques used to build three-dimensional (3D) parts by progressively laying down and binding layers of material to the specification of a digital model, such as a computer-aided design (CAD) model. AM methods have several advantages over traditional, subtractive methods like CNC machining. Instead of having a part made from a mould and have it go through several machining processes, a part can instead be made in a single step. This can reduce the production time and associated costs. Material costs are also limited to the exact material used to create the part, save for if or when support structures are used. AM offers a greater degree of design freedom, as it eliminates many limitations imposed through traditional methods. Parts can be produced on demand and customised easily, allowing features to be modified late in the design cycle if necessary. The origins of additive manufacturing can be traced back to the development of rapid prototyping methods back in the 1980s and 1990s. Initially, these methods were limited to low-strength materials such as polymers and waxes[1], [2]. Eventually, these methods were improved to be able to produce higher quality parts, viable for commercial and industrial use. Other technologies would be developed through the 1990s and early 2000 s, such as electron beam melting (EBM) and selective laser melting (SLM), which would introduce metal processing capability to AM. The history, development and capabilities of rapid prototyping and AM technologies are discussed further in Chapter 2.

The AM market has seen substantial growth, with its worth being estimated to be over $\$ 4$ billion in 2014, [3]. It is expected to grow to over $\$ 21$ billion by 2020 [4]. Whilst a large portion of the market is devoted to polymer-based AM, there has been substantial interest in 
metal-based AM methods, and in particular, powder-bed based systems such as EBM and SLM, also called powder-bed fusion additive manufacturing processes.

Powder-bed fusion AM systems use a high energy delivery system, such as a high-power laser or electron beam, to heat and melt sections of a thin bed of evenly spread metal powder, around $20 \mu \mathrm{m}$ to $200 \mu \mathrm{m}$ in thickness. The powder reaches a high enough temperature to melt, and the resulting melt pool extends to the solid material beneath the powder and binds to it. The melt pool quickly re-solidifies, and the laser moves to other parts of the powder bed to repeat the process. After a layer is completed, the build platform is lowered, and another layer of powder is evenly spread over the previous one, and the process is repeated until the part is completed.

Powder-bed based AM methods have seen widespread use throughout the automotive, aerospace and medical industries, due to their ability to create useable rapid prototyped parts reducing the overall design and production lead costs.

In both automotive and aerospace industries, any small reduction in time and development cost can result in significant overall savings in the development of a vehicle or aircraft. Automotive manufactures have utilised powder-bed fusion AM methods such as selective laser sintering (SLS), laser beam melting (LBM) and SLM for prototyping, as well as the rapid fabrication or repairing of tooling components, [5]-[7]. Additionally, the need for tool manufacture for a production of a part can be cut out entirely by fabricating the part in a single procedure, shortening the design and production cycle [8], [9].

Aerospace industries utilise powder-bed fusion AM methods to create highly complex products with high performance properties. The designer freedom offered by AM eliminates the need for assembly features and allows for the addition of features with internal functionality, such as internal honeycomb structures to reduce weight whilst maintaining mechanical strength, [10]. The manufacturing company Siemens claimed to have successfully created and tested gas turbine blades, made with a revised blade design and improved internal cooling channels [11]. 
Medical industries utilise AM techniques extensively due to the ease in which 3D medical imaging data can be converted into solid objects. Orthopaedic and dental implants can be customised to fit individual patients quite easily using AM techniques [12], [13]. Certain alloys, such as Tantalum, are useful in implants due to their biocompatibility and chemical resistance. However, they are also found to be difficult to process using conventional metal processing techniques due to their high cost and melting temperatures, Powder-bed fusion methods, such as EBM and SLM, are able to process these types of alloys quite readily, and are thus popular and ideal methods to use within the field, [14], [15].

\subsection{Limitations and Issues concerning Powder-Bed Fusion}

Most powder-bed fusion methods typically have low build rates and small build volumes, although there have been efforts by manufacturers to increase both the build volume and build rate. A typical laser powder-bed fusion system uses one fibre laser, ranging between $200 \mathrm{~W}$ to $1 \mathrm{KW}$ capacity, and can achieve build rates of around $5-20 \mathrm{~cm}^{3}$ per hour, in a build volume limited to $250 \mathrm{~mm} \times 250 \mathrm{~mm} \times 325 \mathrm{~mm}$, [16]. Manufacturers of SLM and EBM systems have attempted to address these limitations through implementing multiple laser beams in the process to increase build rate (e.g. the Renishaw RenAM $500 Q^{1}$ ), or continually increasing the build volume offered by their machines (e.g. SLM $500^{2}$ or EOS M $400^{3}$ ). However, the defining limitation, which is often referred to as the Achilles heel of AM, [17], is ensuring part quality and reproducibility, especially for large scale production as desired by the automotive and aviation industries. Laser powder-bed fusion is subject to highly complex and dynamic manufacturing constraints, and it has been estimated that there are nearly 130 influential parameters that can affect the process, [18].

Defects such as porosity within the part, [19],surfaces roughness, [20], and residual stresses [21], [22], cause a reduction in the physical properties of powder-bed fusion based parts. These defects can be attributed to the formation and subsequent solidification of the melt

\footnotetext{
${ }^{1}$ http://www.metal-am.com/renishaw-introduce-four-laser-system-formnext-2017/

$2 \mathrm{https}: / / \mathrm{slm}$-solutions.com/products/machines/selective-laser-melting-machine-slm-500

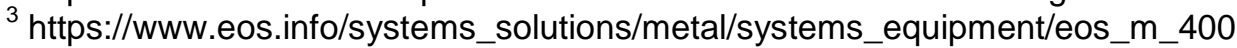


pool. The factors affecting the formation include the laser processing parameters, such as the laser power, beam diameter, scanning pattern and scanning speed, which have a direct impact on how energy is delivered to the metal powder particles. For example, if there is insufficient melting of the powder due to low energy input from the laser, a phenomenon known as balling may occur, where the scanned laser track breaks into a series of droplets. This can have negative affects the part, such as pore formation and surface roughness, [23]. Detrimental issues could arise from the powder bed itself, from differences in the powder alloy composition, size, morphology, particle size distribution and presence of oxidation. Powder re-use has been found to be a potential liability in powder-bed fusion processes, where repeated cycles of powder reuse can cause a reduction the certain elements within the chemical composition of the part [24]. Powder particle size and distribution can affect the flowability and packing density of the powder bed, [25].

\subsection{State-of-the-Art in Generating Process Maps}

As new machines are developed, and new powder materials are introduced, it is important that high throughput methods are developed to identify optimal processing parameters. Such methods should investigate the relationship between the process parameters and formation of the melt pool. Single melt track process maps are a highly utilised method of performing such an investigation, wherein the laser only scans powder in a single, narrow width, consisting of the diameter of the beam.

The process map provides an in-depth study of the interaction between the laser, the powder and the solid substrate. Two commonly used parameters are the laser power (W) and the laser speed $(\mathrm{mm} / \mathrm{s})$. The process map should ideally assist in the selection of optimal laser settings with energy densities which avoid melt-track defects, such as balling. A criticism which has been made of process maps is that they are not general enough, and this is exasperated by a number of factors. One factor is the rapidly developing technology of the AM machines themselves, with changing laser types, beam sizes and powers, powder deposition strategies and gas flow handling. Another factor is the lack of user control of 
machine parameters at a fundamental level to control build sequences, particularly the movement and firing of the laser and stage movements. Often the ported software is developed by other companies, not necessarily the machine manufacturer and is more geared towards simplification of the build preparation for full components rather than providing full access to all machine parameters for scientific study.

In addition to process maps, bulk properties such as density and tensile strength are used to select optimal machine parameters over multiple layers, using Design of Experiments (DOE) and Analysis of Variances (ANOVA). The time taken to determine optimal machine parameters for a new AM powder has come down from 3 to 4 months to a few weeks, and this is typically done looking at bulk density, using optically measured porosity, and mechanical properties. However, this is still too long when multiple iterations are required, for example in the case where multiple new compositions of powders need to be tested and assessed with relatively small amounts of powder.

Recent research has made it possible to envisage in real time the way in which powder particles melt and solidify at the level of the melt-pool, or along tracks [26], [27].

Computational models are being developed and validated, but the complexity of the physics, and the difficulty of observing the melt-pools and limited thermal measurements, makes the modelling of limited use at the moment.

This means that the traditional way of understanding the interaction between the laser, powder and substrate using physical experimentation persists as the preferred means for new powder alloy development and optimisation of machine parameter. However, the combination of factors described above has led to published process maps often being outof-date by the time of publication, tending to make it difficult to establish empirical relationships between beam sizes and powder depth, and linking results to existing knowledge of larger beam sizes, [28] and outcomes from computational models. However, single track experimental work which results in process maps leads to a better understanding of not just the porosity formation mechanisms, but also the inter-layer penetration and the resulting microstructures which are formed and have a direct effect on 
the as-built mechanical properties of the material. Yadroitsev and Smurov, [29], [30], provide examples of the early attempts at generalising rules for melt-pool instabilities, but these were typically done with low laser powers and energy densities. These have been based upon an often-used method of direct melting powder onto base-plates or substrates made through machining traditionally fabricated metal plates. Although these base-plates or substrates may be compositionally similar to the material powder used, they may have a very different microstructure. This puts into question whether the penetration of the melt pool into the plate or substrate is equivalent to what occurs at the powder-bed level of the process.

It has only been relatively recently that the formation of keyhole melt formations has been demonstrated, [26], [31], [32]. These defects are usually avoided by using a suitable selection of machine parameters within pre-selected bands. With a two- to three-fold increase in the available laser powers, it is becoming apparent that there is no simple linear relationship between power and speed which would allow an equivalent increase in build rates, and it may be that at higher laser powers the transition to keyholing may be more difficult to control.

\subsection{Objectives of this work}

The aim of this work is to extend knowledge of the LPBF melt pool development by the creation of a new standard for measuring single-track process maps and structures. Key aspects which need to be met by these experimental techniques should include:

- $\quad$ Capture instabilities and melt-pool profiles as a function of laser power and speed, validated to empirical expectations and previous experimental work.

- $\quad$ Be representative of tracks as they would be laid at multiple layers in the process.

- $\quad$ Not be constrained to any single alloy powder.

- Be 'high throughput' in that they allow not only a complete exploration of machine parameters within a single build, but also, they must be easy to remove from the base plate and ready for rapid metallographic preparation and microscopy. 
- $\quad$ Allow for the exploration of additional parameters such as powder depth in a controlled manner.

\subsection{Publications}

The following two publications feature some the research performed during this study. The first has been published and can be read in Appendix 1. The second publication will be published later this year.

- [1] "Verification of Numerically Calculated Cooling Rates of Powder Bed Additive Manufacturing", HW Mindt, M Megahed, NP Lavery, A Giordimaina, SGR Brown, TMS 2016 145th Annual Meeting \& Exhibition, 205-212

- [2] "Validation and optimisation of a new high-throughput Crucible method for single-line melt-pool characterisation", A. Philo, S. Sillars, A.Giordimaina, S. Mehraban, S.G.R. Brown, N.P. Lavery, To be published in Journal of Materials Processing Technology, 2018 


\section{Chapter 2 Overview of Additive Manufacturing Systems}

\subsection{Introduction}

The standards organisation ASTM International (American Society of Testing Materials) has defined Additive Manufacturing, [33], as follows:

“... A process of joining materials to make objects from 3D model data, usually layer upon layer, as opposed to subtractive manufacturing methodologies. Synonyms: additive fabrication, additive processes, additive techniques, additive layer manufacturing, layer manufacturing, and freeform fabrication"

The variety of manufacturing methods that fall under this definition are numerous as is the range of useable materials; including polymers, ceramics, composites and metals These materials can be delivered in multiple forms, such as liquid, microscopic powder, granular powder or wire form.

In this chapter, a detailed explanation and analysis for three different types of metal-based additive manufacturing methods are discussed, followed by a more in-depth discussion of the equipment used during this research.

\subsection{Electron Beam Melting}

Electron beam melting (EBM) is a powder bed-based AM process, similar to SLM, where an electron beam is used as a heat source to melt or sinter material to create 3D objects. Unlike the previously discussed AM methods (stereolithography and fused deposition modelling), EBM can be used to process a wide range of metal materials, such as titanium alloys, aluminium alloys, cobalt-base alloys, steel and copper, [34]-[39]. However, EBM is not used with ceramic and plastic materials, as EBM requires electrically conductive material to function. The EBM process takes place under high vacuum conditions, typically between $10^{-}$ ${ }^{4}$ and $10^{-2}$ Torr, [37], and as such the EBM process has the added benefit of reducing oxidation. 
EBM, like other AM processes, allows much more freedom for design than other conventional fabrication processes. This allows for the creation of parts with highly complex structures and geometries and reduced build weight. EBM-made parts can achieve near $100 \%$ density and have mechanical properties comparable to or sometimes better than ascast or wrought parts without post-processing, [38], [40], [41]. The EBM process has received growing interest and has seen increasing applications in the aerospace and automotive industries, [42]-[44]. EBM has the ability to process biocompatible alloys, and has thus generated applications in the medical and dental industries, [45], [15], [46], [47]. EBM utilises one or more electron beams to melt the metal powder bed. This constant stream of electrons is created using an electron emitter, such as a heated tungsten filament, kept under high vacuum conditions $\left(10^{-5}\right.$ Torr). The emitter and the beam require vacuum conditions as electrons would interact with gas molecules otherwise, decreasing the efficiency of the process. Working under vacuum reduces the risk of contamination of the melt pool and oxidation, as previously stated.

A high voltage is passed through the filament, causing thermal electron emission to occur. A stream of electrons is ejected from the filament, and it is directed as a beam towards the powder bed using inertia-free electromagnetic lenses. The lack of moving mechanical parts allows the beam to move almost instantaneously from point to point, reaching speeds of up to $10^{5} \mathrm{~ms}^{-1}$, [48]. The kinetic energy from the electrons in the beam is transferred and converted into thermal energy as it interacts with the metal powder bed, causing the powder particles to melt and coalesce or sinter together.

The powder bed must be initially heated by scanning the electron beam several times over the entirety of the bed. This causes the powder to sinter slightly, improving the electric conductivity and helping prevent the repulsion of charged powder particles, [49]. The powder particles are separated from each other after the build is complete by abrasive blasting, using the same powder material as the blasting agent. Pronounced necking produced by sintering is not present, and the powder can usually be almost completely recycled and reused. 
A variant of the EBM process is electron beam freeform fabrication, where the material can be introduced in the form of a continuous wire, which is fed into the melt pool under an electron beam. Multiple wires of different materials or composition can be used to produce functionally graded parts or parts with custom alloy compositions. This method is found to be nearly $100 \%$ efficient in feedstock consumption as well as achieving $95 \%$ power usage efficiency, [50].

\subsection{Laser Metal Deposition}

Laser metal deposition (LMD) is a type of AM method in which the powder material is fed into a high-power laser beam using a nozzle system. A number of different technologies utilise this method, such as laser engineering net shaping (LENS), [51], direct metal deposition (DMD), [52], laser net shape manufacturing (LNSM), [53], and others. The nozzle is coaxially or laterally oriented to the incoming beam, as depicted in Figure 1. This special apparatus can be fitted unto a CNC system or robotic arm, allowing the material to be delivered freely in any orientation [54]. The nozzle delivers the powder in an inert gas, such as nitrogen or argon, to minimize oxidation. The materials used during LMD include various metal alloy powders such as Ti-6Al-4V or Inconel 718, [55], [56], A small molten pool is generated from this process and deposited unto a substrate which it becomes fused to. More powder may be drawn into the melt pool to increase the size of the deposited metal. Tracks are closely placed to one another in an overlap configuration and on top of another until the object geometry is completed. 


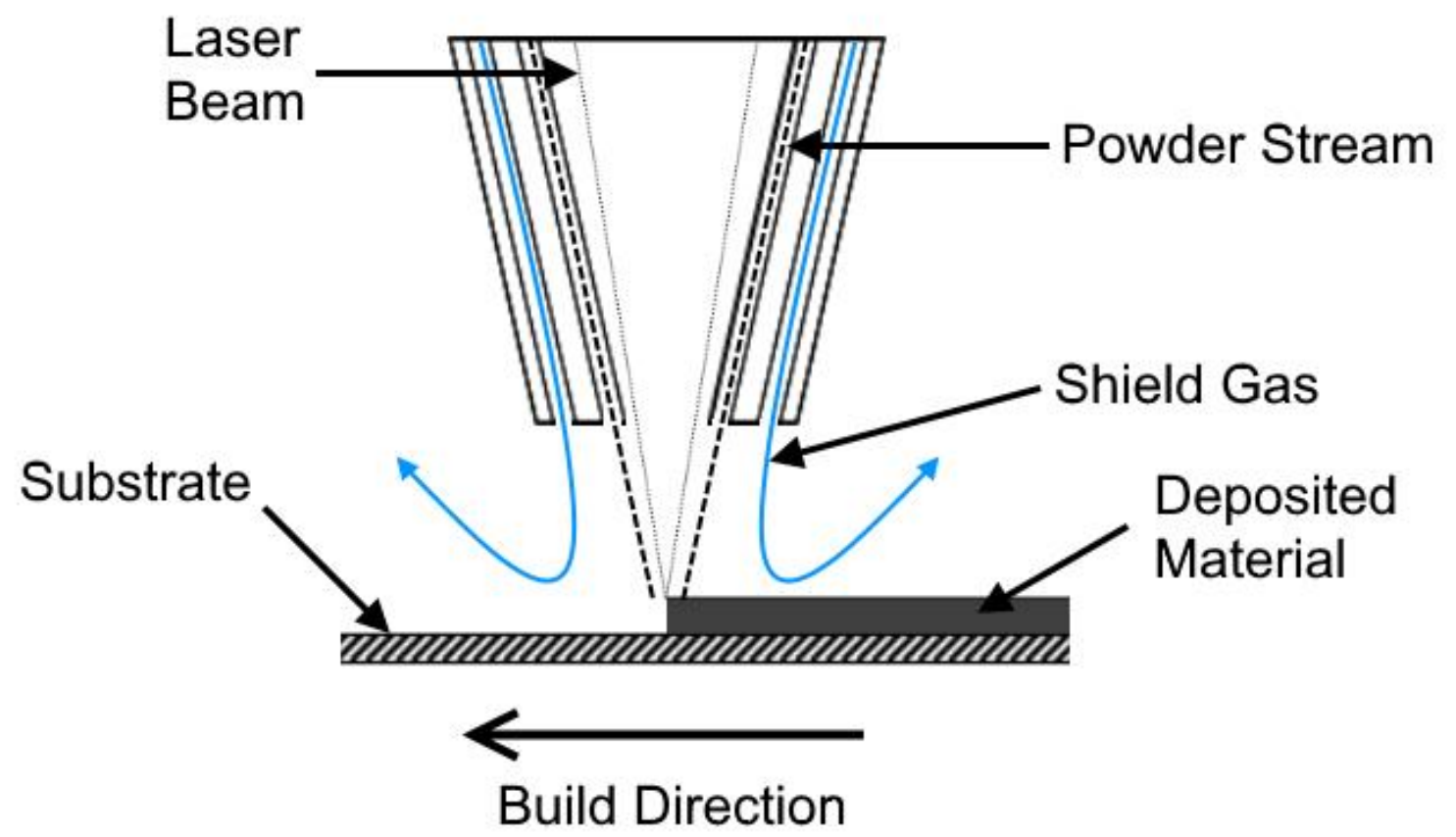

Figure 1. Laser Metal Deposition Apparatus.

LMD is a highly versatile technology. Apart from being able to manufacture new objects, it can also be used to repair or rebuild worn or damaged components and add a corrosion or wear resistant coating to existing objects, [57].

\subsection{Selective Laser Melting}

Selective laser melting (SLM) is a manufacturing technique most similar to laser sintering or EBM, utilising a laser source to fully melt metallic powders to create functional, complex parts with $99.9 \%$ density without the need of post-processing. Using this method, parts can be made with mechanical and material properties matching or of better quality than parts made using traditional manufacturing.

Hardness values and elastic modulus of magnesium parts produced by SLM have been found to be comparable to those of cast ingots, [58]. Commercially pure titanium parts manufactured by SLM, often used to make biomechanical implants, were found to possess better mechanical properties compared to parts produced by traditional processing technologies such as casting and machining, [19]. Apart from the improvement in 
mechanical properties such as microhardness, compressive and tensile strengths, SLM offers a higher degree of freedom in designing such parts with almost no geometric constraints. Similarly, Al-12Si parts manufactured by SLM were found to possess yield and tensile strengths respectively four and two times higher than corresponding values for cast material, [59].

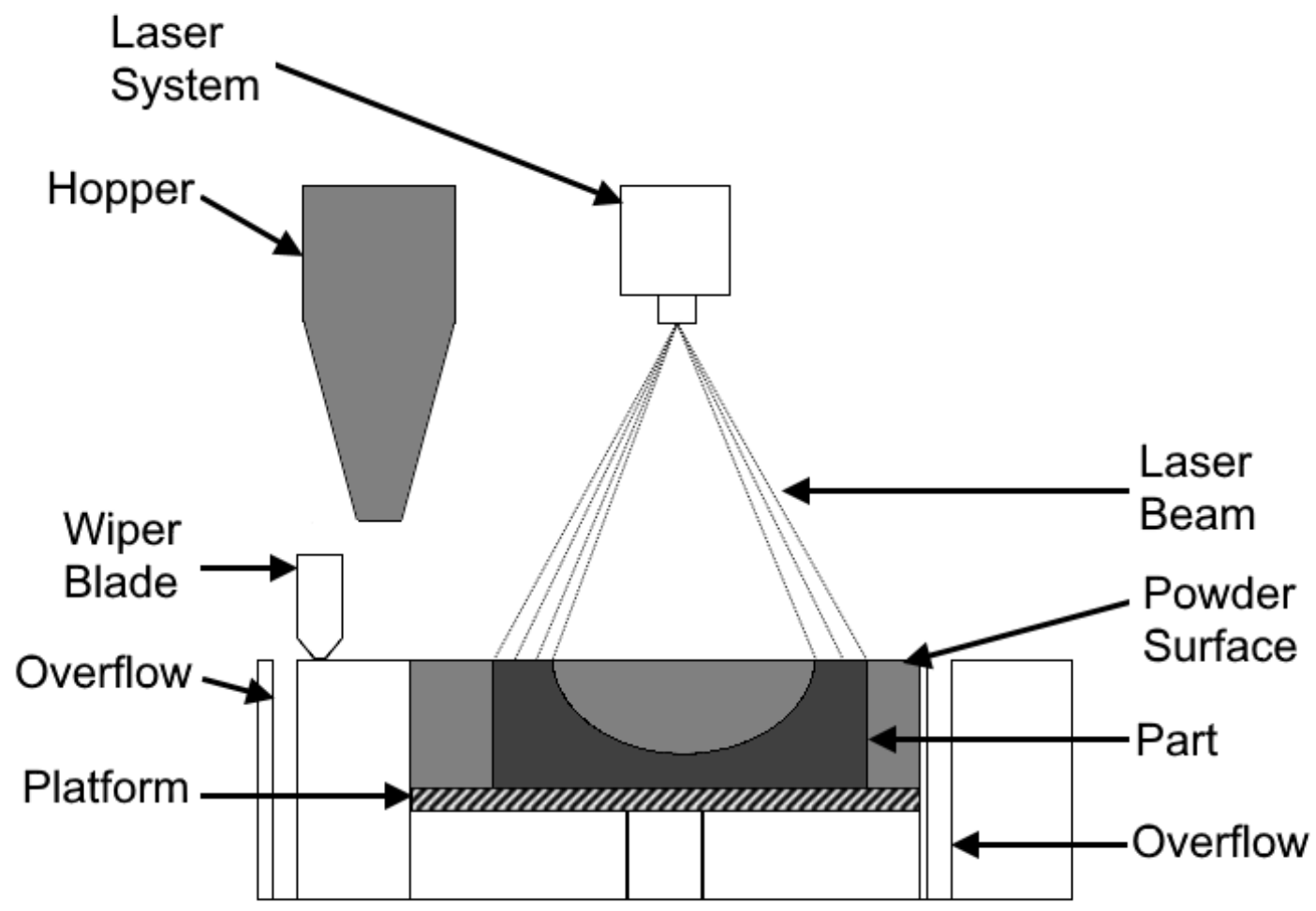

Figure 2. Schematic drawing of SLM process.

It is important for the atmosphere within the chamber to be inert. The presence of reactive gases, such as oxygen, have detrimental effects on the build and powder quality. Certain alloying elements, such as manganese, chromium, titanium, aluminium and silicon have a high affinity for oxygen and are commonly used in many powder materials. Oxidation, decarburisation and other problems that can impact the mechanical properties of the part are reduced by using a vacuum pump to remove air from the build chamber and replace it with a non-reactive gas such as nitrogen, helium or argon. The possibility of ignition and 
combustion of more volatile material powders such as pure titanium is also prevented in the presence of an inert atmosphere.

Typically, in most SLM systems, a single high-power laser beam is used. However, there are systems that utilise two or more lasers to increase the build rate of the process, such as SLM solutions SLM500, which uses four fibre lasers. Nd:YAG, Yd:YAG and $\mathrm{CO}^{2}$ are commonly used lasing mediums. The lasing medium used is important as they produce beams with differing wavelengths, which are more readily absorped by different materials as shown in Figure 3.

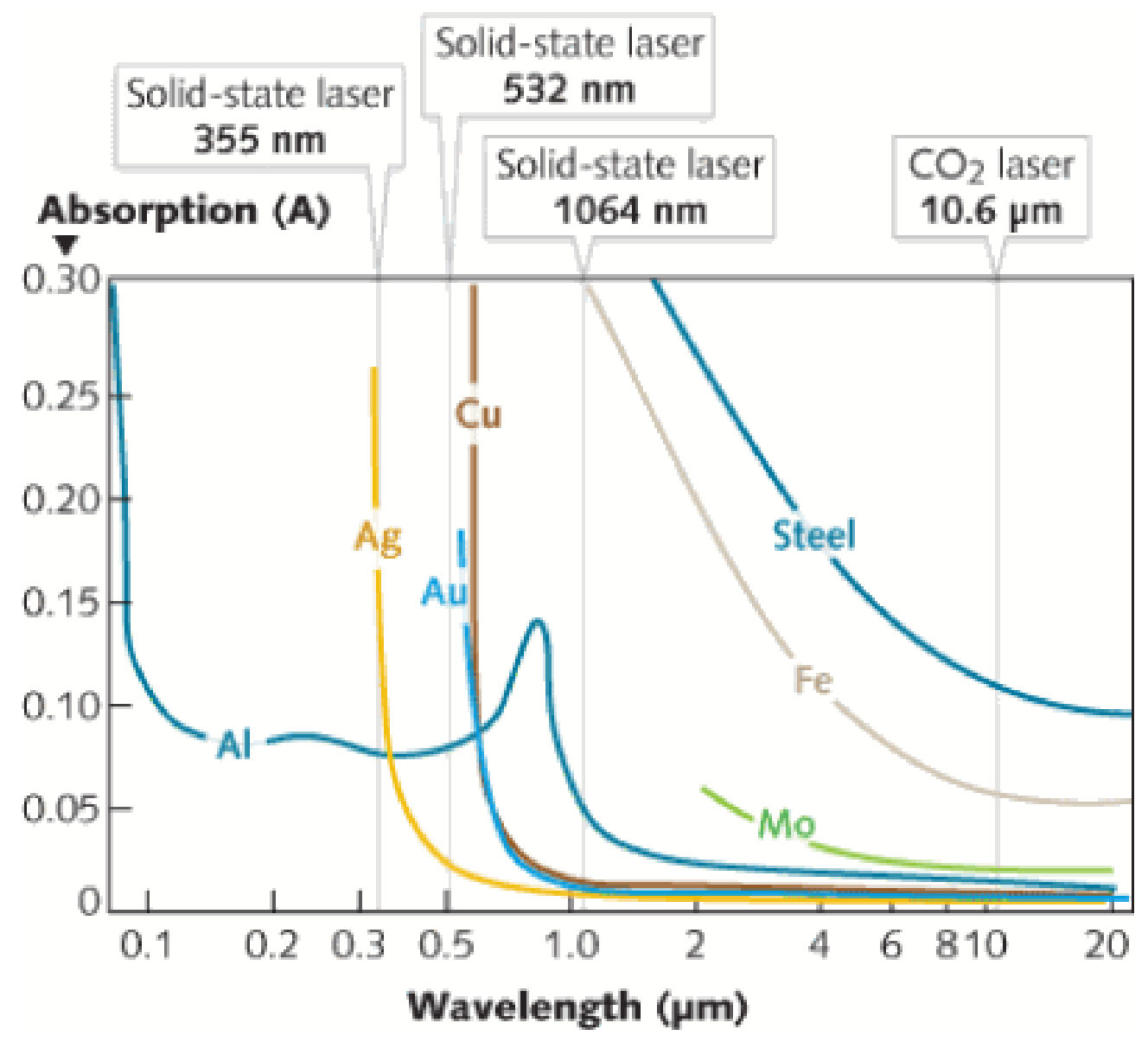

Figure 3. Absorption of laser output at different wavelengths, [60].

A high-speed mirror galvanometer is used to direct the laser onto the powder bed. The galvanometer controls the speed, position and size of the laser beam, which is lastly directed into an $\mathrm{f}-\theta$ lens. An $\mathrm{f}-\theta$ lens is designed with built-in barrel distortion that allows the position of the laser spot to be altered using the product of the focal length $(F)$ and the tangent of the 
deflection angle $(\theta)$. This greatly simplifies the positioning algorithms required to direct the laser.

Powder can be fed from a hopper mounted above the build area or a powder feed container that deliver powder from below the build area. Either delivery method deposits enough powder for a thin layer, which is spread and levelled over the build area by a roller or wiper blade. After laser treatment of the layer of powder, the build platform is lowered by a single layer thickness and the depostion and spreading process is repeated. Any excess powder is pushed into a pair of crevices, one located at the end of the build and the other behind the hopper, which lead to an overflow container. This container can fill up during long build times, and must be regularly emptied to prevent overflow powder backing up into the build area.

The main processing parameters are laser power $(P)$, point distance $(\mu \mathrm{m})$, hatch spacing $(\mu \mathrm{m})$, focal spot diameter $(\mu \mathrm{m})$, exposure time $(\mu \mathrm{s})$, scan speed $\left(\mathrm{mms}^{-1}\right)$, layer thickness $(\mu \mathrm{m})$ and scan strategy. Laser power is set and limited by the laser hardware. In certain systems the laser is not applied continously but is moved in a discrete manner from one point to the next. The point distance is the distance between two successive laser points, whilst the hatch spacing is the distance between two consecutive lines in a hatch pattern. The diameter of the laser spot can be altered by changing the distance from the laser focus plane and the powder bed, as shown in Figure 4. The exposure time is the amount of time the laser spends on each point. The scan speed can be determined by considering the ratio between point distance and exposure time. The laser off-time between successive points is not accounted for using this ratio. The layer thickness is the distance the build platform descends after finishing a single layer. The scan strategy is the pattern followed by the laser for every layer in the horizontal plane. The pattern used in the scan strategy is usually offset by increments in this angle between successive layers. 


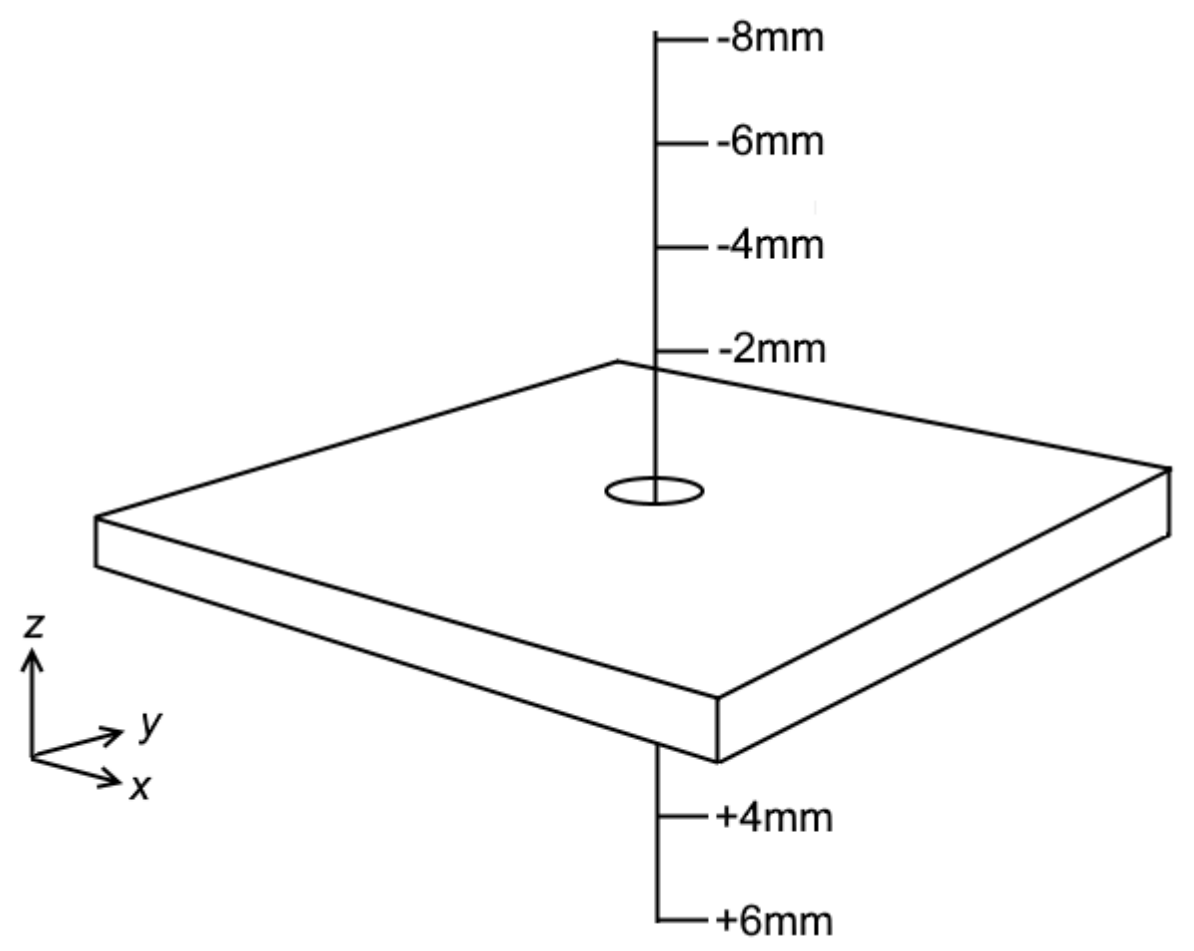

Figure 4. Laser focus positions with respect to the powder bed.

Features on the same layer such as the core, border and skin are given different parameters to achieve appropriate properties to the in-layer section. For example, the skin region, which is the region that makes up the surface of the part, will require different build properties from the core region which makes the bulk of the part. These properties improve the surface quality of the part. This scan strategy works well for regions of the surface facing along the zaxis, known as upskin and downskin, resulting in a reduction in overall roughness and giving a smooth surface finish. Other properties include an offset region, border regions and regions dedicated to overhangs.

Four commonly used scanning patterns are the stripe, checkerboard, islands and meander. The stripe pattern divides the layer into bands of single tracks divided by a hatching distance. Each band can overlap with the next. The scan direction remains the same from layer to layer, but the band changes position slightly. The checkerboard and island patterns divide the layer into a patchwork of squares of a fixed side length, resembling a checkerboard. The line direction in each square is perpendicular to its neighbouring square. 
When the squares are built in a random order, this method is referred to as the islands pattern. When the equivalent of the white squares, corresponding to a checkerboard layout, is built first, followed by the black squares, this method is referred to as the checkerboard pattern. In a meander pattern, the scan direction for every line is the same. The pattern is rotated by a fixed angle for every layer.

\subsection{Lasers}

Light, radiant heat and other forms of radiation can be described as electromagnetic disturbances in the form of waves that propagate through the electromagnetic field. Light describes the way in which radiant energy is carried through space and time, [61]. It has a dual nature, wherein light can act both as a wave and as a particle, referred to as a photon, [62].

$$
E=h f=\frac{h c}{\lambda}
$$

$\mathrm{E}=$ speed of light $\left(\mathrm{m} \mathrm{s}^{-1}\right)$

$h=$ Planck's constant $\left(6.63 \times 10^{34} \mathrm{Js}\right)$

$\mathrm{f}=$ frequency of the radiation $(\mathrm{Hz})$

$\lambda=$ wavelength of the photon $(\mu \mathrm{m})$

LASER is an acronym of "light amplification by stimulated emission of radiation". Lasers are used to emit light that is amplified and with the same wavelength, phase and direction. There are three central components that make up a laser: the lasing medium, the energy pump and an optical cavity. The basic operating mechanisms of a laser are shown in Figure 5.

When the lasing medium gets excited by energy, light is emitted in all directions. The lasing medium can be in the form of a gas, liquid or semi-conducting material. The energy pump provides excited electrons to the lasing material. Mechanisms include electricity from a power supply, flash tubes, lamps or energy from another laser. The optical cavity reflects light from the lasing medium back into itself. It usually consists of two mirrors, one at each end of the lasing material. The light generated from the lasing material is reflected between the two mirrors, increasing the strength of the beam via amplification of the energy from the 
excitation mechanism in the form of light. A partially transparent mirror on one end of the lasing material allows some light to leave the optical cavity to be used for the production of the laser beam.

Energy is delivered to the lasing medium from the pump, and is stored in the form of electrons within the atoms or molecules of the medium. These electrons are elevated to different quantum levels, or energy states that are usually unstable. Electrons in unstable energy states release the energy back as photons almost immediately, returning back to a ground state. The wavelength of the photons emitted is determined by the energy levels of the electrons. In some materials, such as those used as a lasing medium, the electrons achieve a metastable state, wherein the atom or molecule remains excited for a longer time. Before laser action can occur, energy must be pumped to the lasing medium until most of the atoms or molecules are in the metastable state rather than the grounded state. This is called population inversion. 


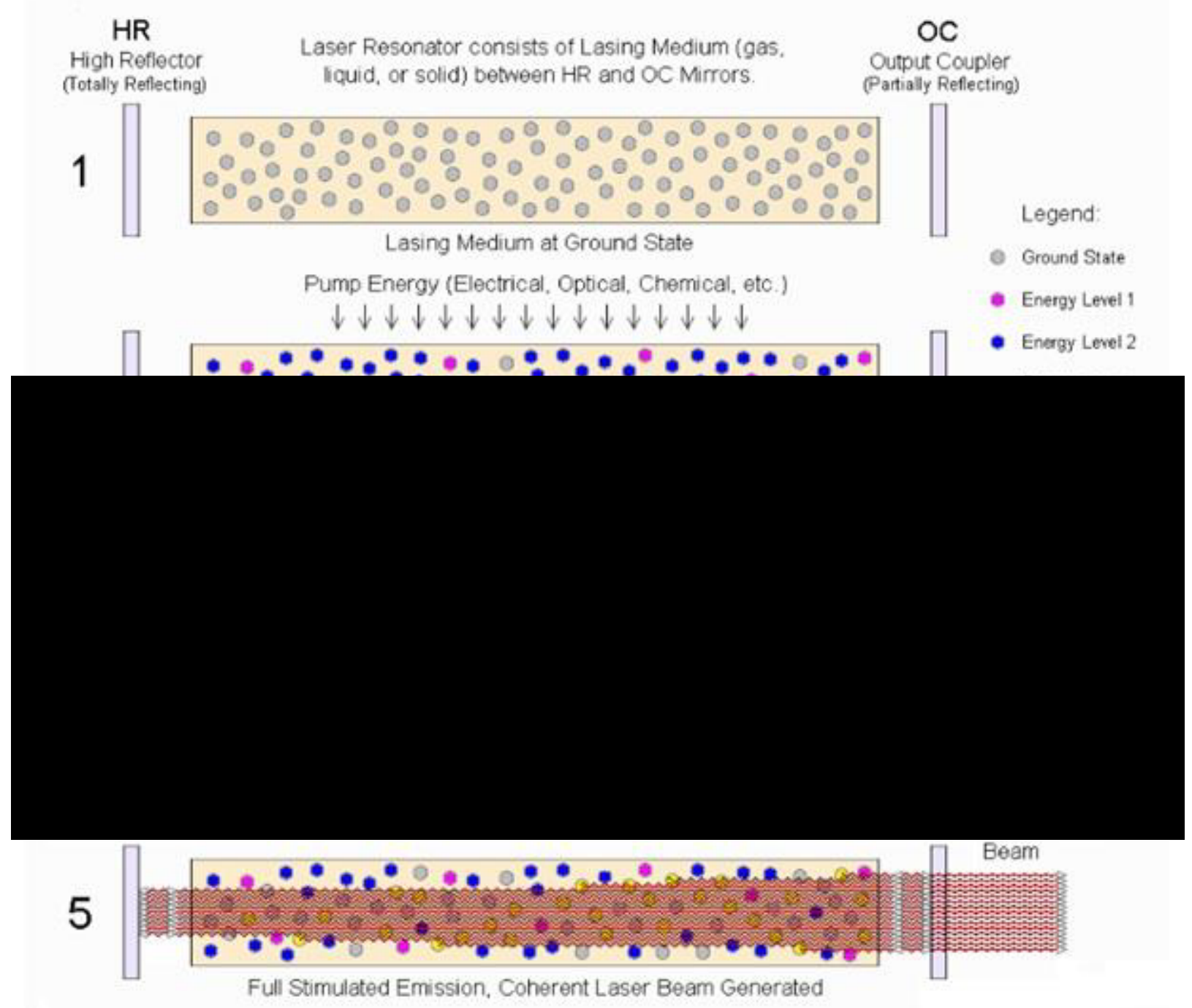

Figure 5. Basic Laser Operation, [63].

The spontaneous decay of a few metastable electrons to lower energy levels occurs. The photons emitted cause a chain reaction, reacting with the remaining metastable electrons. The photons released from this chain reaction have precisely the same wavelength, phase and direction as the previous photons. This action occurs in the optical cavity.

Most of the photons are lost, but those that decay in the direction of the mirrors reach the end of the lasing medium and are reflected back into the material. This continues the chain reaction and more photons are released. A portion of the photons that arrive at the partially reflecting mirror emerge as the laser beam, whilst the rest are reflected back into the cavity. $\mathrm{Nd}$ : YAG (linear formula $\mathrm{Nd}: \mathrm{Y}_{3} \mathrm{Al}_{5} \mathrm{O}_{12}$ ) is used as a crystalline, semi conductive solid state lasing material. Triple ionised neodymium replaces yttrium ions within the structure of an 
yttrium aluminium garnet to alter the conductivity of the crystal, and provides the lasing capability to the material. Nd:YAG produces light at a wavelength of $1064 \mathrm{~nm}$, which is one order of magnitude smaller than $\mathrm{CO}_{2}$ lasers. This short wavelength is more readily absorbed by metallic components and is thus widely used in manufacturing industry for uses such as cutting and welding of steels and drilling of super alloys used for gas turbines.

\subsection{Renishaw Systems}

The Renishaw AM line of systems use metal powder bed fusion technology, as classified by ASTM international. They produce metal parts from a bed of fine metal powders, the diameter of which should range between 15 microns and 45 microns. The systems can handle a wide range of metal powders, including titanium alloy Ti-6Al-4V, cobalt chromium CoCr, aluminium alloy AISi10Mg, stainless steel 316L and nickel alloy Inconel 625. Such machines allow additional material to be loaded into the machine whilst it is still running.

\subsection{AM250 Specifications}

All the work undertaken for this thesis was done on a Renishaw AM250, pictured on the lefthand image in Figure 6, installed at Swansea University in 2012. The AM250 has a 250mm by $250 \mathrm{~mm}$ by $300 \mathrm{~mm}$ build envelope, with a $250 \mathrm{~mm}$ by $250 \mathrm{~mm}$ build plate which can be heated to $140^{\circ} \mathrm{C}$, which is shown on the right-hand image in Figure 6 .

The laser used in the AM250 is a 200W Ytterbium fibre laser operating at a wavelength of $1070 \mathrm{~nm}$ and modulated with a frequency of $100 \mathrm{kHz}$. The laser spot size is typically $70 \mu \mathrm{m}$, and the layer thickness controlled by the z-stage which can move with an accuracy of $\pm 2 \mu \mathrm{m}$ gives layers between $20 \mu \mathrm{m}$ and $70 \mu \mathrm{m}$, but typically set at $50 \mu \mathrm{m}$. Quoted build speeds ranged from $5 \mathrm{~cm}^{3}$ to $20 \mathrm{~cm}^{3}$, depending on material, with maximum $X$ - and $Y$ - scanning speeds of up to $2000 \mathrm{~mm} / \mathrm{s}$.

The original machine was initially supplied with the MTT AUTOFAB software. As of recent, in 2017, Renishaw also supplied a number of working licences for QuantAM build preparation 
software, a state-of-the-art software package designed specifically for use with Renishaw AM machines.

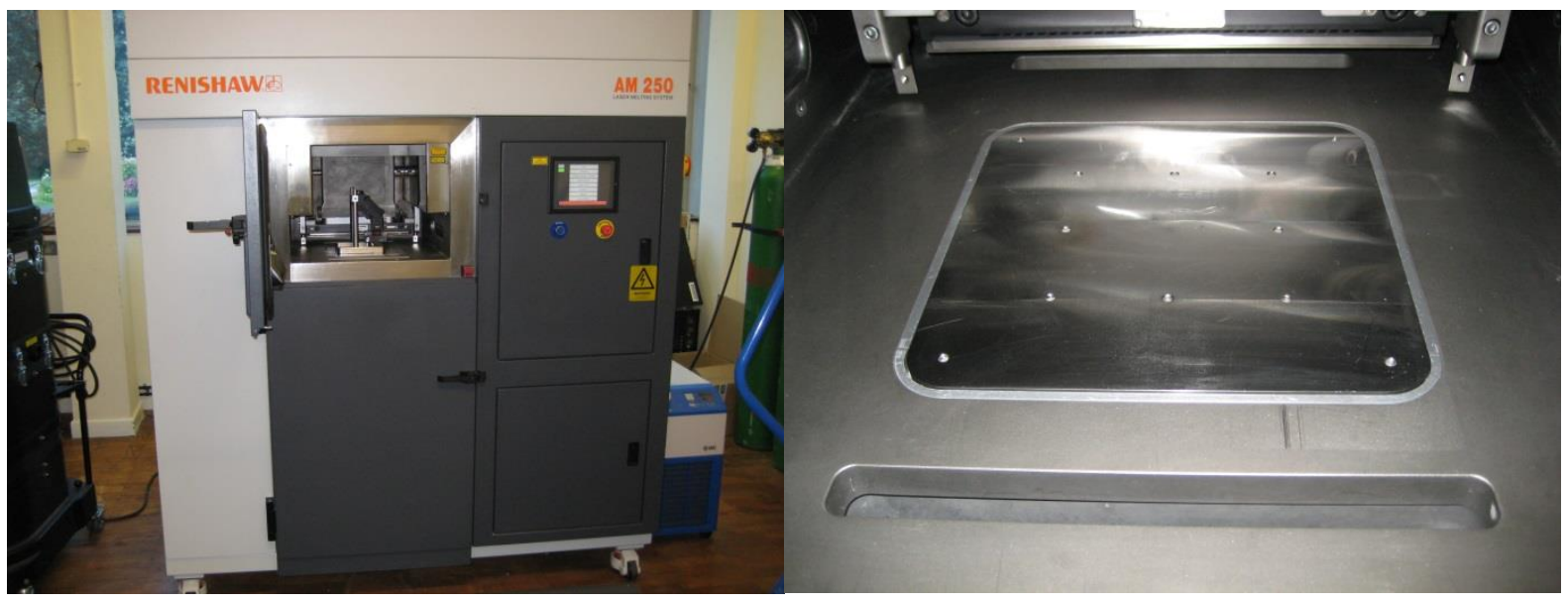

Figure 6. AM250

Left) The Renishaw AM250 Right) Z-drive to which baseplate is attached.

The results of a calibration test using a beam profiler for a variable focus selection of $70 \mu \mathrm{m}$ are shown in Table 1 below. From this it can be seen that the beam spot size at the $0 \mathrm{~mm}$ focal point is slightly elliptical with a minor axis ( $x$-direction) of $66.46 \mu \mathrm{m}$, and a major axis ( $y$ direction) of $72.02 \mu \mathrm{m}$.

\begin{tabular}{|c|c|c|c|c|}
\hline $\begin{array}{c}\text { Focus, } \\
(\mathbf{m m})\end{array}$ & $\begin{array}{c}\text { Average X, } \\
(\boldsymbol{\mu m})\end{array}$ & $\begin{array}{c}\text { Spot Size } \\
\text { Increase }\end{array}$ & $\begin{array}{c}\text { Average Y, } \\
(\boldsymbol{\mu m})\end{array}$ & $\begin{array}{c}\text { Spot Size } \\
\text { Increase }\end{array}$ \\
\hline $\mathbf{5}$ & 126.23 & $14.76 \%$ & 148.3862069 & $-30.4 \%$ \\
\hline $\mathbf{4}$ & 110.00 & $18.46 \%$ & 133.06 & $33.7 \%$ \\
\hline $\mathbf{3}$ & 92.86 & $27.23 \%$ & 99.53 & $18.2 \%$ \\
\hline $\mathbf{2}$ & 72.98 & $5.48 \%$ & 84.21 & $1.1 \%$ \\
\hline $\mathbf{1}$ & 69.19 & $4.10 \%$ & 83.31 & $15.7 \%$ \\
\hline $\mathbf{0}$ & 66.46 & & 72.02 & \\
\hline $\mathbf{- 1}$ & 67.37 & $1.36 \%$ & 71.16 & $-1.2 \%$ \\
\hline $\mathbf{- 2}$ & 69.64 & $3.38 \%$ & 71.31 & $0.2 \%$ \\
\hline $\mathbf{- 3}$ & 74.80 & $7.41 \%$ & 76.32 & $7.0 \%$ \\
\hline $\mathbf{- 4}$ & 84.66 & $13.18 \%$ & 83.14 & $8.9 \%$ \\
\hline $\mathbf{- 5}$ & 102.57 & $21.15 \%$ & 92.55 & $11.3 \%$ \\
\hline $\mathbf{- 6}$ & 118.4965517 & $15.53 \%$ & 99.53103448 & $7.5 \%$ \\
\hline
\end{tabular}

Table 1. Spot size calibration of the Renishaw AM250 used in this work.

The laser is controlled by $\mathrm{X}$ - and $\mathrm{Y}$ - galvanometers which re-direct the laser beam down through an F-theta lens which focusing the beam onto the base plate. The main parameters which are controlled in the AM250 are the point distance (d1 in Figure 7), the hatch spacing 
(d3 in Figure 7), the laser power (W) and the exposure time ( $\mu \mathrm{s})$. Additional parameters related to the scan pattern (or hatch pattern) are shown in Figure 7. The contour of the area being printed at a given layer is sometimes surrounded by a border (or skin) in which slightly different parameters are used to provide a smoother surface finish. This border is called the volume border (labelled d4 in Figure 7). Between the border and the main internal area (called the volume area) is a small volume offset hatch (labelled d2 in Figure 7). The laser will typically traverse the volume border as a single line around the entire contour, after completing the internal volume area.

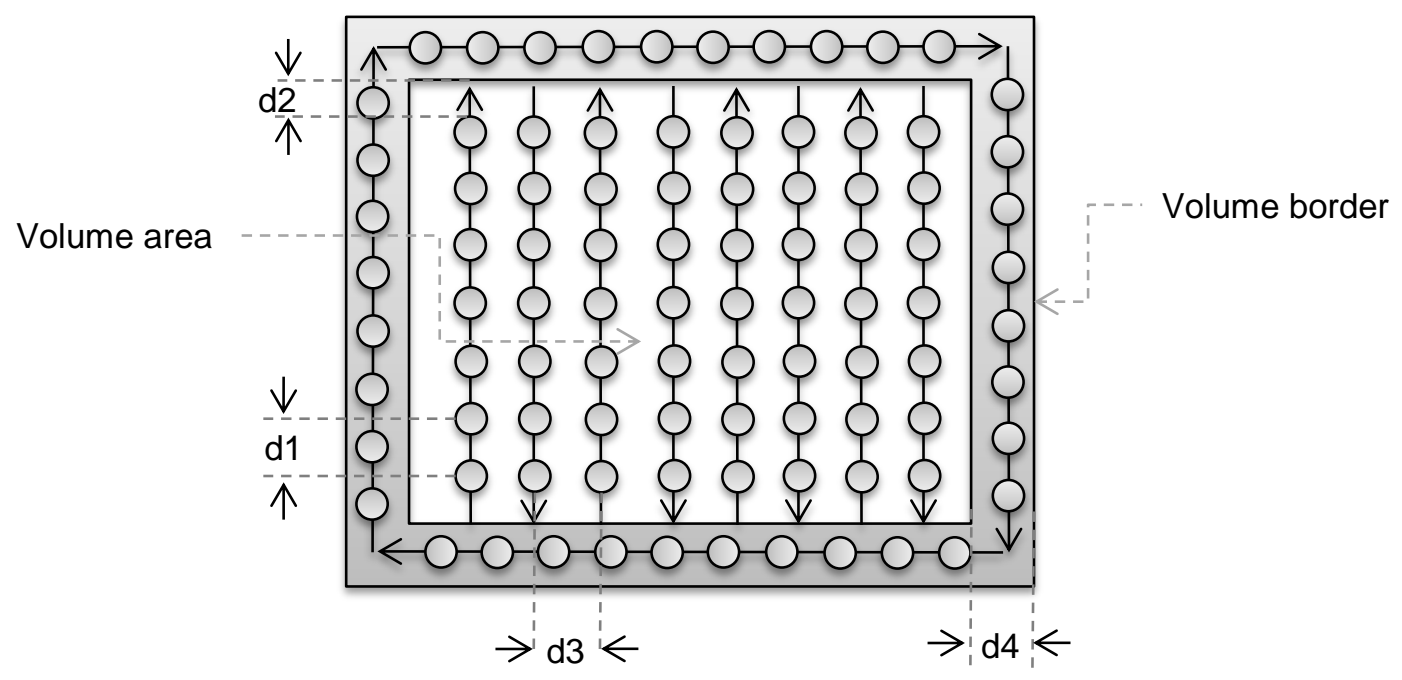

Figure 7. Laser parameters in the Renishaw AM250.

There are various ways in which the laser scan pattern (or hatch pattern) can be selected to fill the volume area. The most commonly used hatch pattern shown in Figure 7 is the meander hatch pattern where the laser leaves one side of the contour travels to the other side and then reverses direction. An alternative scan pattern of the laser can be achieved using stripes in the same direction, and the entire area can be divided into patches The typical ranges of these parameters are given in Table 2. 


\begin{tabular}{|c|c|c|}
\hline & Volume area & Volume border \\
\hline Point distance $(\mu \mathrm{m})$ & $50-150$ & $50-80$ \\
\hline Exposure time $(\mu \mathrm{s})$ & $50-150$ & $60-150$ \\
\hline Power $(\mathrm{W})$ & $100-200$ & $90-200$ \\
\hline Hatch spacing $(\mu \mathrm{m})$ & $50-150$ & N/A \\
\hline
\end{tabular}

Table 2. Typical laser parameters for the Renishaw AM250.

Together, the point distance and the exposure time determine the scan speed of the laser (if one ignores small transitional speeds due to galvanometer movement) by

$$
\text { Scan speed }=1000 \times \frac{\text { point distance }}{\text { exposure time }}
$$

Where, point distance is measure in $\mu \mathrm{m}$ and exposure time is measured in $\mu \mathrm{s}$. Using the parameters in Table 2 gives scan speeds ranging from $100 \mathrm{mms}^{-1}$ to $3000 \mathrm{~mm}^{-1}$.

\subsection{Conclusions}

This chapter has given a brief introduction and background to Additive Manufacturing, particularly in the context of powder bed fusion systems capable of process metal powder material. In particular, the Renishaw AM250 laser powder-bed fusion system was discussed, which was used throughout the work in the coming chapters. 


\section{Chapter 3 Thermo-Mechanics of melt pool formation}

\subsection{Introduction}

Ideally, parts produced by laser-powder bed fusion should be fully dense and have comparable or improved mechanical and microstructural properties to those produced using traditional methods. Additionally, this should be accomplished without the need of lengthy or expensive post-processing. The powder material that is subjected to the laser must undergo complete melting in order to reduce the possibility of pore or defect formation, [64]. Careful control over the process parameters is required in order to obtain the best possible physical properties from parts part made using powder material.

In order to melt the metal powder and maintain a suitable build speed, the energy input provided by the laser must be high enough to melt the powder in a short amount of time, in the order of microseconds. The energy from the laser quickly dissipates from the spot of laser-powder interaction, and the powder undergoes a solid-liquid-solid transition in a very short amount of time, resulting in a very steep thermal gradient. This can lead to residual stress formation, crack formation and distortion in the part, [65]-[68], [30], [22].

If the energy input is too high, it can cause vaporisation of the material, causing a distinct type of pore formation known as keyholing, [26], [32], [69], [70], [31]. Conversely, if the energy input is too low, this can cause insufficient melting and wetting of the powder, which can lead to instability of the melt and so called "balling" of the melt bead, [22], [31], [71]-[76]. These phenomena are linked to pore formation, surface roughness and can even cause the laser-powder bed fusion process to malfunction.

In this chapter, the laser-powder bed fusion process was reviewed in detail, investigating the varying heat transfer mechanisms that take place, the solid-liquid-solid transition cycle of the powder material, and the possible defect and instability formation that may take place during this cycle. 


\subsection{The Physical Model}

In this section, the formation of the melt pool using a high-power laser beam will be discussed in detail and chronologically. A simplified diagram of the physical phenomena taking place has been given in Figure 8. Generally, all the heat transfer mechanisms involved fall under three types; convection, conduction and radiation.

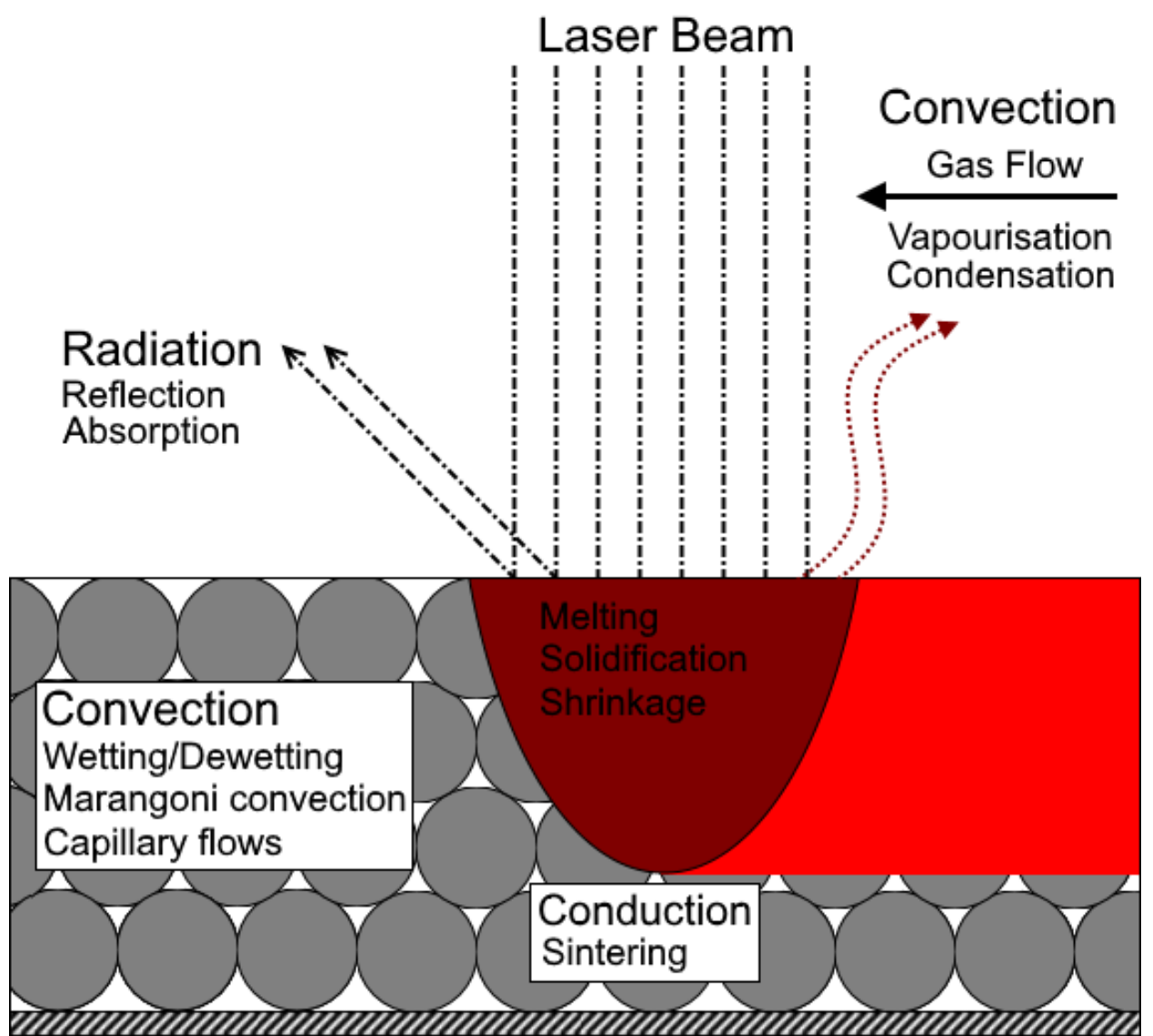

Figure 8. Physical phenomena at play in the powder-bed fusion system.

\subsubsection{The Powder Bed}

Before the melt pool can be formed, a powder bed and substrate (known as the base plate) must be present. The physical attributes and composition of powder used for laser powderbed fusion can be just as influential to the build and completed parts as the processing parameters used by the machine. Physical characteristics of the powder material, such as particle size, shape and size distribution, influence the formation and topology of the powder 
bed, which subsequently influences the production of the part. Other factors, such as the storage and recycling of the powder material, can also influence the properties of the built parts due to oxidation.

Spherically shaped powder particles are preferred in powder-bed based AM methods, as they have improved flowability and help form a uniform powder bed, [77], [78]. Conversely, non-spherical particles decrease compaction in the powder bed, leading to increased porosity in parts, [52]. The gas atomization method is a commonly used process for creating spherically shaped metal powder material. This method is particularly preferred as it uses an inert gas to create the powder particles, which significantly prevents oxidation of metals, such as stainless steel. During this process, molten metal is hit by a high-speed jet of inert gas, such as argon, making the metal form into spherical droplets, [79]. The droplets cool to below their melting temperature as they pass through a cooling tower, they solidify and are screened and sorted by their size.

The powder is packaged to have a certain range of powder sizes and a set particle size distribution. Very fine particles, with sizes between $0.1 \mu \mathrm{m}$ and $20 \mu \mathrm{m}$, are avoided as they can form clusters which are detrimental to powder flowability. They are also more likely to combust or explode upon making contact with a reactive gas due to their large surface area to volume ratio, [25].

The particle size distribution defines the relative frequency of particles, by mass, of the given range of sizes. This determines the flowability of the powder and the packing density of the powder bed. A balanced particle powder size distribution improves the packing density of the powder bed, allowing smaller particles to fill the gaps in the as-deposited layer, [80]. The maximum particle size in the distribution would determine the minimum layer thickness that can be used, [25].

\subsubsection{The Laser Beam}

The wavelength and power of the laser is determined by the type of laser beam used, and should be adapted to the powder as discussed in 2.5 The radiation emitted is typically either 
absorbed by the material or reflected off the surface. The degree of absorption is governed by the emissivity of the surface which the radiation interacts with. Emissivity is defined as the ratio of energy radiated from a materials surface to that radiated by a perfectly emitting material, known as a blackbody. Emissivity is a dimensionless number, ranging between 0 to 1 , where 0 is a perfectly reflecting body whilst 1 is a perfectly emitting body. The emissivity of a metal surface increases with the surface roughness of material, the level of oxidation present, and its temperature, [81]-[83]. The emissivity of the materials involved will change as laser interaction continues. A molten pool has higher levels of reflectivity than loose powder, [84], [85].

Due to the porous nature of the powder bed, radiation absorption is higher than that of bulk, solid metal. Laser radiation undergoes multiple reflections on the spherical surface of the powder material inside the pores of the bed, [86]. This phenomenon is known as multiple scattering, and the degree of scattering depends on the powder bed formation. A study by Boley et al., [87], demonstrated through a ray-tracing model that absorption can be increased by a factor of 2 by using an optimised powder structure.

\subsubsection{Melting of the Powder Bed}

The energy from the beam not reflected from the surface is absorbed by the powder bed, causing its temperature to rise. Thermal energy is transferred between particles by heat diffusion. Once the temperature exceeds the solidus temperature of the metal, the solid-fluid phase transformation begins. Further energy input is required, known as latent heat of fusion, to fully complete the transformation. The specific latent heat of fusion of a material is the heat energy required to change $1 \mathrm{~kg}$ of a solid material at its melting point to $1 \mathrm{~kg}$ of liquid, without changing its temperature.

$$
E_{m}=\boldsymbol{m} H
$$

Where $E_{m}$ is the energy required to melt to material $(\mathrm{J}), m$ is the mass $(\mathrm{kg})$ of the material and $H$ is the specific heat capacity of the material $\left(\mathrm{Jkg}^{-1}\right)$. 
The law of heat conduction, or Fourier's law, states that "the heat flux resulting from thermal conduction is proportional to the magnitude of the temperature gradient and opposite to it in sign", [88].

\subsection{Effects}

As previously mentioned in Chapter 2, there are several processing parameters controlling the laser melting process. Each parameter has a direct influence on the formation of the molten pool, and the degree of influence the parameters have on one another is not always apparent. Apart from the parameters of the machine itself, the material properties of the powder metal itself also imposes an influence on the formation of the melt. Control over the flow and consistency of the melt tracks tends to be more difficult for standard alloy powders with inherent narrow melting temperature ranges, [89], than for specially developed alloys or powder mixtures with wider temperature ranges, [90].

\subsubsection{Wetting and Balling}

Wetting is defined as the ability of a liquid to form an interface with a solid surface. The degree of wetting of a liquid can be measured by its wetting angle $(\theta)$ with the surface it is in contact with. The smaller the wetting angle, the greater the degree of wetting, as shown in Figure 9 below. For maximum adhesion and perfect wetting, the liquid must completely cover the surface $\left(\theta=0^{\circ}\right)$. As the wetting decreases, the contact angle would increase. At large contact angles $\left(\theta \geq 90^{\circ}\right)$, conditions for wetting on the surface are considered highly unfavourable. 


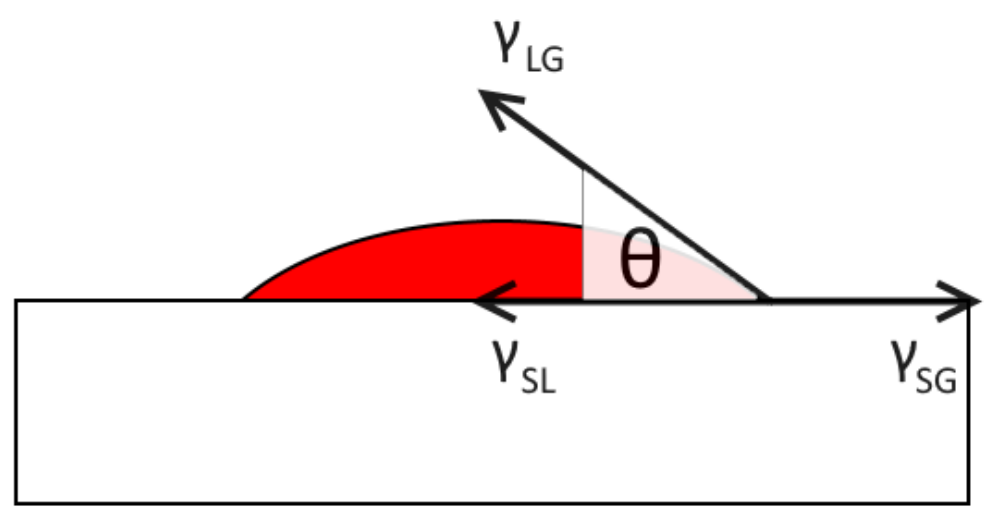

Figure 9. Surface Wetting

Young's equation, show in below, defines the mechanical equilibrium of a liquid drop in contact with a completely solid surface, under the action of three interfacial tensions [91].

$$
\gamma_{S G}=\gamma_{S G}+\left(\gamma_{S G} \times \cos \theta\right)
$$

YLG, YSL and YsG represent the liquid-gas, the solid-liquid and the solid-liquid interfacial tensions, respectively. The units for these parameters are $\mathrm{Nm}^{-1}$.

One detrimental phenomenon that can occur during laser melting is the break-up of the track during the molten phase of the powder bed-laser interaction. This is referred to as balling, as the molten material can break up into a series of isolated spherical droplets, although it should be noted that the molten track can break up into larger shapes as well. The degree of balling is influenced the wettability of the melt, which is influenced by several parameters and conditions, such as the oxygen content in the build atmosphere, [73], [92], the size and shape of powder , [93], and the influence of laser power and scan speed , [29], [73], [94], [95].

Balling occurs when the forces of surface tension are more influential than the wetting and spreading of the melt. For a volume of a liquid, each molecule in the entirety of the liquid body is pulled equally in every direction by the neighbouring liquid molecules, resulting in a zero-net force. At the surfaces of the liquid, however, the molecules at such surfaces do not have neighbouring molecules in every direction to provide the balanced net force. Instead, they are pulled into the liquid body by their neighbouring molecules, creating an internal 
pressure, called surface tension. The liquid therefore contracts its surface area to maintain the lowest surface energy value, and as a result the liquid takes a sphere or spherical shape. In practice, external forces such as gravity further deform the droplet, and consequently the wetting angle is affected by a combination of surface tension, gravity, surface roughness and fluid flow, such as those caused by capillary forces.

\subsubsection{Plateau-Rayleigh Instability}

This phenomenon is named after Joseph Plateau and Lord Rayleigh, a Belgian physicist and an English physicist, respectively. Joseph Plateau first observed instability of a liquid during an experiment in 1873. When a column of water is suspended vertically, it falls under the influence of gravity as a jet. When the length of the column exceeds the diameter by a factor of around 3.13, the water no longer assumes the shape of a column and instead breaks down into a stream of droplets, [96]. The water breaks up in this way as to reduce the total surface energy of the stream.

Intramolecular forces within the liquid pull equally in all directions except at the surface, where they can only pull along the surface. There is a net inward cohesive force, which acts as a driving factor in minimising the total area of the liquid, making it take a spherical shape. The force present within the surface layer of a liquid is called surface tension. It is defined as the work required per unit area $\left(\mathrm{Jm}^{-2}\right)$ to create and maintain the new surface. A column of water has a much higher surface area compared to a stream of spherical droplets of the same total volume, so a lower energy state is achieved with the formation of droplets. This value is dependent on the material properties of the liquid as well as the temperature of the surface. In general, surface tension decreases with an increase in temperature. The molecules within the liquid vibrate at a higher frequency with an increase in thermal energy, reducing the cohesive forces between liquid molecules. The net inward cohesive force thereby decreases as well, decreasing the overall surface tension.

The break-up by surface tension forces is gradual, rather than being instantaneous. In 1878, Lord Rayleigh showed theoretically that a cylinder of water would deform into varicose 
perturbations, [97]. These would take the form of sinusoidal periodic displacements, as shown in Figure 10.

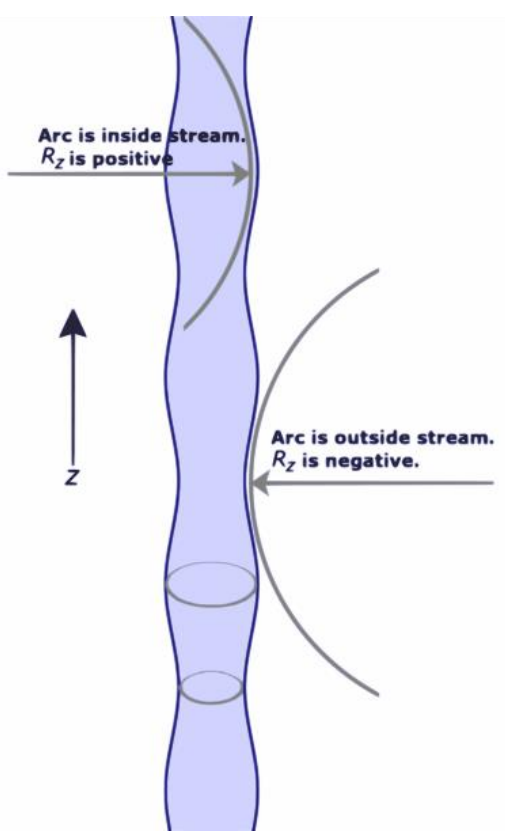

Figure 10. Plateau-Rayleigh Instabilities, [98].

The radius of the column is no longer constant, and the column breaks up into a series of narrow and wide sections. The wide sections experience low pressure whilst the narrows sections experience high pressure, causing a pressure gradient to form that in turn causes fluid flow. The flow causes the displacement amplitude to increase. Once the wavelength of these displacements exceeds the circumference of the column, the narrow sections rupture. The wider sections assume the shape of spherical droplet, achieving the lowest energy state.

\subsubsection{Marangoni Convection}

Marangoni convection, also known as the Gibbs-Marangoni effect, is a form of fluid flow that takes place where there is a gradient of surface tension at an interface between two phases. A liquid-gas interface is a very common instance where it would take place. Flow is driven from regions with a low surface tension to regions of high surface tension, [99]. The gradient in surface tension can be caused by changes in the chemical composition (solutocapillary 
effect), electric potential (electrocapillary effect) or temperature (thermocapillary effect). Any mixture of the effects can also occur simultaneously.

Since selective laser melting is a very high energy process, thermocapillary flow is the most influential phenomena affecting Marangoni flow within the melt. During the process, the centre of the melt pool tends to be at a higher temperature than at the edge. Thus, an additional force is exerted from the hot centre to the cooler edges, causing balling to occur (i.e. a break up of an elongated liquid region into individual balls of material).

In selective laser melting, when metal powder is melted due to laser processing, the stream of liquid formed from laser interaction is subject to the same phenomena. A steep thermal gradient is achieved between the centre and edge at the surface of the melt pool. Some key considerations are:

- Compressibility and viscous forces are negligible.

- Specific system geometry depends on energy minimisation.

- Liquid desires to be in minimal energy state.

For high energy processes like laser-powder bed fusion, a steep thermal gradient is developed between centre and edge of metal pool at surface. Surface tension is a function of temperature, and the presence of a large temperature gradient induces a Marangoni flow from regions of low surface tension to regions of high surface tension, [99], i.e. from the edge of the melt pool to its centre. This flow of fluid produces an additional force in the melt pool, which is exerted onto the molten bead and positively influences the balling phenomenon.

Fluid flow will produce an extra force which exerts itself on the molten track of laser-powder bed fusion samples and positively influences the balling phenomenon.

The stability of a liquid during laser-powder bed fusion depends on the laser power, scanning speed, powder layer thickness, substrate material, physical properties and granulomorphometry of the powder used. Stability zones are characterised by formation of 
stab pools and continuous tracks. Instability zones are characterised by non-continuous tracks and individual droplet formation.

Plateau-Rayleigh instability causes peaks and troughs to form along a track. At troughs, the melt pool height is low and it takes less time for the substrate to cool this region as there is less material present. At peaks the melt pool height is high. More material and therefore heat is present, and the liquid phase persists for longer. Connections between peaks and front part of flow breaks at troughs and they start acting like bottlenecks. Temperature profiles on the surface melt and substrate are influenced by melt topology. Temperature in the substrate decreases more quickly under troughs and increases under peaks. With such a nonmonotonous behaviour, the surface cools unevenly. Temperature field evolution is perhaps the most important parameter in laser-powder bed fusion.

Capillarity and wetting are strongly correlated and are both governed by surface and melt interface energies. This depends on experimental conditions and whether the liquid wets solid powder or re-solidified material from the melt pool.

\subsubsection{Keyholing}

The conditions for keyhole mode melting have been studied in laser welding applications, which are very similar to the powder-bed fusion process, [100]. Keyhole mode melting is known to be detrimental in the laser-powder bed fusion process due to pore formation [101]. Due to the Gaussian profile of the laser beam, the highest energy density is concentrated towards the centre of the beam, whilst the edges of the beam are lower. As a result, the temperature of the resulting metal pool follows this profile, and the resulting temperature gradient drives thermocapillary flow towards the centre of the melt pool and deeper into the substrate. Additionally, the high energy density at the centre of the pool can cause temperatures to rise above boiling point, causing vaporisation of the metal.

The recoil pressure from the vapour exerts a force onto the molten pool, causing a cavity to form within the melt. The cavity can additionally cause the laser to be reflected multiple times below the melt pool surface, effectively increasing its efficiency and allowing deeper 
penetration into the substrate by the laser and melt pool. This is a similar phenomenon to multiple scattering within the powder bed, as described in Chapter 3.2.2. Once the laser moves away from the melt pool, the molten material in the upper part of the melt pool fills the void under the force of gravity. The vapour in the void becomes trapped in the lower part as the surface of the melt pool cools quickly when exposed to the inert gas atmosphere. The voids caused through this phenomenon would be spread through the track, causing severe pore formation in the part. 


\section{Chapter 4 Experimental Methods and Materials}

\subsection{Optimal Density Parameters}

\subsubsection{Introduction}

For each different batch of material powder used during the single-layer experiments, a Design of Experiments (DOE) method was performed to find their individual optimal build parameters. The purpose of a DOE is to apply a statistics-based experiment to determine the relationship between the input parameters and the resulting outputs. In this research, the output that determined optimal input parameters was density.

The input parameters chosen for laser-powder bed fusion were laser power $(\mathrm{W})$, point distance $(\mu \mathrm{m})$, hatch spacing $(\mu \mathrm{m})$ and exposure time $(\mu \mathrm{s})$. The nominal laser spot diameter of $70 \mu \mathrm{m}$ was kept constant in each DOE method. The parameters were varied according to an orthogonal array, which would be used to create different combinations of the input parameters. In this research, one of two orthogonal arrays were used, either the L9 array or the L25 array.

Energy density was calculated for each parameter combination. Energy density is a parameter that measures the energy input from the laser to the powder bed, and it is calculated using the following equation[102], [103]:

$$
E_{d}=\frac{P}{v \times h \times t}
$$

where $E_{d}$ is the energy density $\left(\mathrm{Jmm}^{-3}\right), v$ is scan speed $\left(\mathrm{mms}^{-1}\right), h$ is hatch spacing $(\mu \mathrm{m})$ and $t$ is layer thickness $(\mu \mathrm{m})$. This equation is suited for continuous lasers, and since the laser used by the Renishaw AM250 is pulsed, the equation was modified as follows:

$$
E_{d}=\frac{P \times\left(\frac{T}{h \times p}\right)}{\text { layer thickness }}
$$

where $T$ is exposure time $(\mu \mathrm{s})$ and $p$ is point distance $(\mu \mathrm{m})$. 


\subsubsection{General Method}

Two DOE methods were used for the stainless steel $316 \mathrm{~L}$ experiments, one for experiments $A$ and $B$, and one for experiments $C$ and $D$. This was due to a change to new material after the previous material had run out on the AM250 additive manufacturing machine between the two pairs of experiments. The differences in processability between batches of the same material is an important practical consideration when using additive powder-based fusion, as there can be a change in optimal parameters between the two batches due to... For the Ti6Al4V Titanium Alloy experiment, only a single batch of powder was used, hence only a single DOE method was performed prior to the experiment.

For each parameter combination, three repetitions of density measurement cubes, all of which made with 12mm sides, were built using a Renishaw AM250 machine. These cubes were removed from the plate after the build was completed and the bulk density of each cube was measured using three gravimetric methods:

1. The sides of the cubes were measured using callipers to determine the approximate dimensions on each side. The volume was calculated from these dimensions, and divided by the cube's weight.

2. The Archimedes principle was utilised by weighing the cube in and out of distilled water with a modified weighing scale.

3. The Archimedes principle was utilised using a Sigma 700/701 tensiometer.

The average bulk density was recorded for each combination of parameters by performing a minimum of three repetitions using each of these three gravimetric methods. The values were then used to plot the average relative density of the cubes against the laser energy density used to fabricate them.

The following sections specify the methods used for each batch of powder material. Each one varied slightly from the other. With every subsequent DOE method performed, new variable input parameters or larger orthogonal arrays were introduced as a means of capturing a more accurate set of input values for achieving maximum bulk density. 


\subsubsection{Stainless Steel 316L Powder (Experiments $A$ and B)}

In the following section, the results from a study carried out by Lavery et al., [104], were used to determine the optimal settings for the batch a the batch of $316 \mathrm{~L}$ stainless steel powder used to during experiments $A$ and $B$. Two parameters, the point distance $(\mu \mathrm{m})$ and exposure time ( $\mu \mathrm{s})$, were varied using an L9 orthogonal array as seen in Table 3.

The nominal settings for $316 \mathrm{~L}$ stainless steel powder, as recommended by the machine manufacturers, were to use a point distance of $65 \mu \mathrm{m}$, a laser exposure time of $110 \mu \mathrm{s}$, a laser power setting of $180 \mathrm{~W}$, a hatch spacing of $124 \mu \mathrm{m}$ and a layer thickness of $50 \mu \mathrm{m}$. These settings would give a scan speed of $590 \mathrm{mms}^{-1}$ during the build. The nominal parameters were used in the experiment, listed as B2 in Table 3.

\begin{tabular}{|c|c|c|c|c|c|c|c|c|c|}
\hline \multirow[b]{3}{*}{$\begin{array}{l}\text { Exposure } \\
\text { time }(\mu \mathrm{s})\end{array}$} & \multicolumn{9}{|c|}{ Point Distance } \\
\hline & \multicolumn{3}{|c|}{ A $-25 \mu \mathrm{m}$} & \multicolumn{3}{|c|}{ B $-65 \mu \mathrm{m}$} & \multicolumn{3}{|c|}{$C-105 \mu \mathrm{m}$} \\
\hline & Sample & $\begin{array}{l}\text { Scan } \\
\text { Speed } \\
\left(\mathrm{mms}^{-1}\right)\end{array}$ & $\begin{array}{l}\text { Energy } \\
\text { Density } \\
\left(\mathrm{Jmm}^{-3}\right)\end{array}$ & Sample & $\begin{array}{l}\text { Scan } \\
\text { Speed } \\
\left(\mathrm{mms}^{-1}\right)\end{array}$ & $\begin{array}{l}\text { Energy } \\
\text { Density } \\
\left(\mathrm{Jmm}^{-3}\right)\end{array}$ & Sample & $\begin{array}{l}\text { Scan } \\
\text { Speed } \\
\left(\mathrm{mms}^{-1}\right)\end{array}$ & $\begin{array}{l}\text { Energy } \\
\text { Density } \\
\left(\mathrm{Jmm}^{-3}\right)\end{array}$ \\
\hline 70 & A1 & 357 & 81.29 & B1 & 928 & 32.27 & C1 & 1500 & 19.35 \\
\hline 110 & A2 & 227 & 127.74 & B2 & 590 & 49.13 & $\mathrm{C} 2$ & 954 & 30.41 \\
\hline 150 & A3 & 166 & 174.19 & B3 & 433 & 65.41 & C3 & 700 & 40.49 \\
\hline
\end{tabular}

Table 3. Design of experiments used for Chapter 4, [104].

The material powder used for Experiments $A$ and $B$ was made via gas atomisation. The nominal particle size ranged from $15 \mu \mathrm{m}$ to $45 \mu \mathrm{m}$, and the specification and the actual chemical composition of the powder is shown in Table 4.

\begin{tabular}{|c|c|c|c|c|c|c|c|c|c|c|c|c|}
\hline Element & $\mathrm{Fe}$ & C & $\mathrm{Si}$ & $\mathrm{Mn}$ & $P$ & $S$ & $\mathrm{Cr}$ & $\mathrm{Ni}$ & Mo & $\mathrm{N}$ & $\mathrm{Cu}$ & $\mathrm{O}$ \\
\hline Minimum & Bal & - & - & - & - & - & 17.5 & 12.5 & 2.25 & - & - & - \\
\hline Maximum & & 0.03 & 0.75 & 2 & 0.025 & 0.01 & 18 & 13 & 2.5 & 0.1 & 0.5 & 0.1 \\
\hline Actual & & 0.019 & 0.67 & 1.45 & 0.019 & 0.006 & 17.9 & 12.7 & 2.36 & 0.06 & 0.2 & 0.022 \\
\hline
\end{tabular}

Table 4. Composition, in weight percentage, of the 316L powder used in the study, [104].

The actual powder size distributions were as follows, D10 $=18.86 \mu \mathrm{m}, \mathrm{D} 50=29.21 \mu \mathrm{m}$, $\mathrm{D} 90=45.10 \mu \mathrm{m}$. These terms are a commonly used metric for describing particle size distribution, known as D-values. They specify the diameter of spherical particles that exist 
within a percentage of the mass from the powder sample taken. For example, the D50 value indicates that $50 \%$ of the sample's mass is comprised of particles with a diameter less than $29.21 \mu \mathrm{m}$. The density measurement cubes were arranged on the base plate as seen in Figure 11. The results from this experiment are presented and discussed in detail in Chapter 5.1 .1

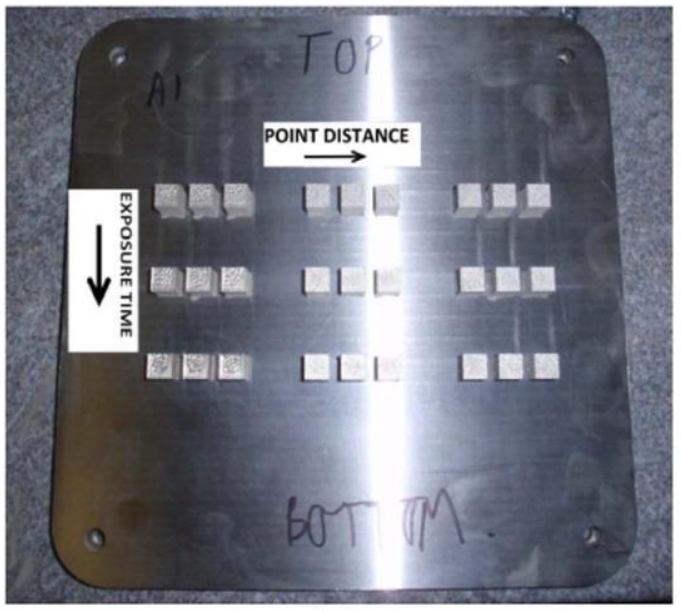

(a)

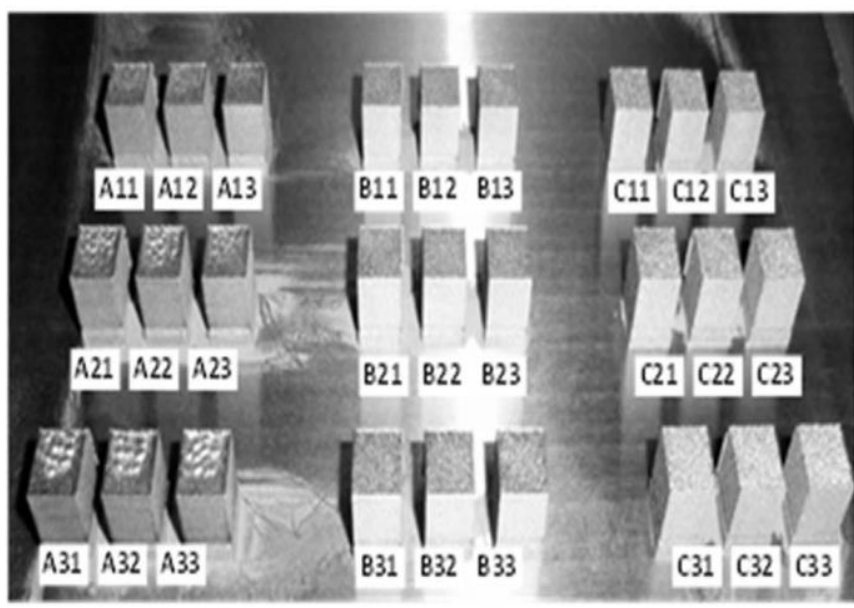

(b)

Figure 11. Right) Layout of the machine parameter array on the build plate, Left) as-built sample labels, [104].

\subsubsection{Stainless Steel 316L Powder (Experiments $C$ and D)}

In the following section, a slightly different DOE method was used on the batch of stainless steel 316L powder used during experiments C and D. An L9 orthogonal array was used again, however two additional variable input parameters was introduced, laser power(W) and hatch spacing $(\mu \mathrm{m})$, in order to investigate their effect on output bulk density. The other two variable parameters were point distance $(\mu \mathrm{m})$ and exposure time $(\mu \mathrm{s})$. The optimal settings recommended by the manufacturers were labelled 'Opt'. The settings used are shown in Table 5. The density cube samples were assembled as seed in Figure 12. Other experimental components can be seen in this image, but they are not relevant to this particular study. The results from this experiment are presented and discussed in detail in Chapter 5.1.2 


\begin{tabular}{|c|c|c|c|c|c|c|}
\hline $\begin{array}{c}\text { Sample } \\
\text { Label }\end{array}$ & $\begin{array}{c}\text { Laser } \\
\text { Power } \\
(\mathbf{W})\end{array}$ & $\begin{array}{c}\text { Point } \\
\text { distance } \\
(\boldsymbol{\mu \mathrm { m } )}\end{array}$ & $\begin{array}{c}\text { Hatch } \\
\text { Spacing } \\
(\boldsymbol{\mu m})\end{array}$ & $\begin{array}{c}\text { Exposure } \\
\text { Time }(\boldsymbol{\mu s})\end{array}$ & $\begin{array}{c}\text { Energy } \\
\text { Density } \\
\left(\mathbf{J m m}^{-3}\right)\end{array}$ & $\begin{array}{c}\text { Scan } \\
\text { Speed } \\
\left(\mathbf{m m s}^{-1}\right)\end{array}$ \\
\hline A1 & 170 & 60 & 100 & 100 & 57 & 600 \\
\hline A2 & 180 & 60 & 124 & 110 & 53 & 545 \\
\hline A3 & 190 & 60 & 149 & 120 & 51 & 500 \\
\hline A4 & 190 & 65 & 100 & 110 & 64 & 591 \\
\hline A5 & 170 & 65 & 124 & 120 & 51 & 542 \\
\hline A6 & 180 & 65 & 149 & 100 & 37 & 650 \\
\hline A7 & 180 & 70 & 100 & 120 & 62 & 583 \\
\hline A8 & 190 & 70 & 124 & 100 & 44 & 700 \\
\hline A9 & 170 & 70 & 149 & 110 & 36 & 636 \\
\hline Opt & 180 & 65 & 124 & 110 & 49 & 591 \\
\hline
\end{tabular}

Table 5. Laser parameters used for DOE for Chapter 5.
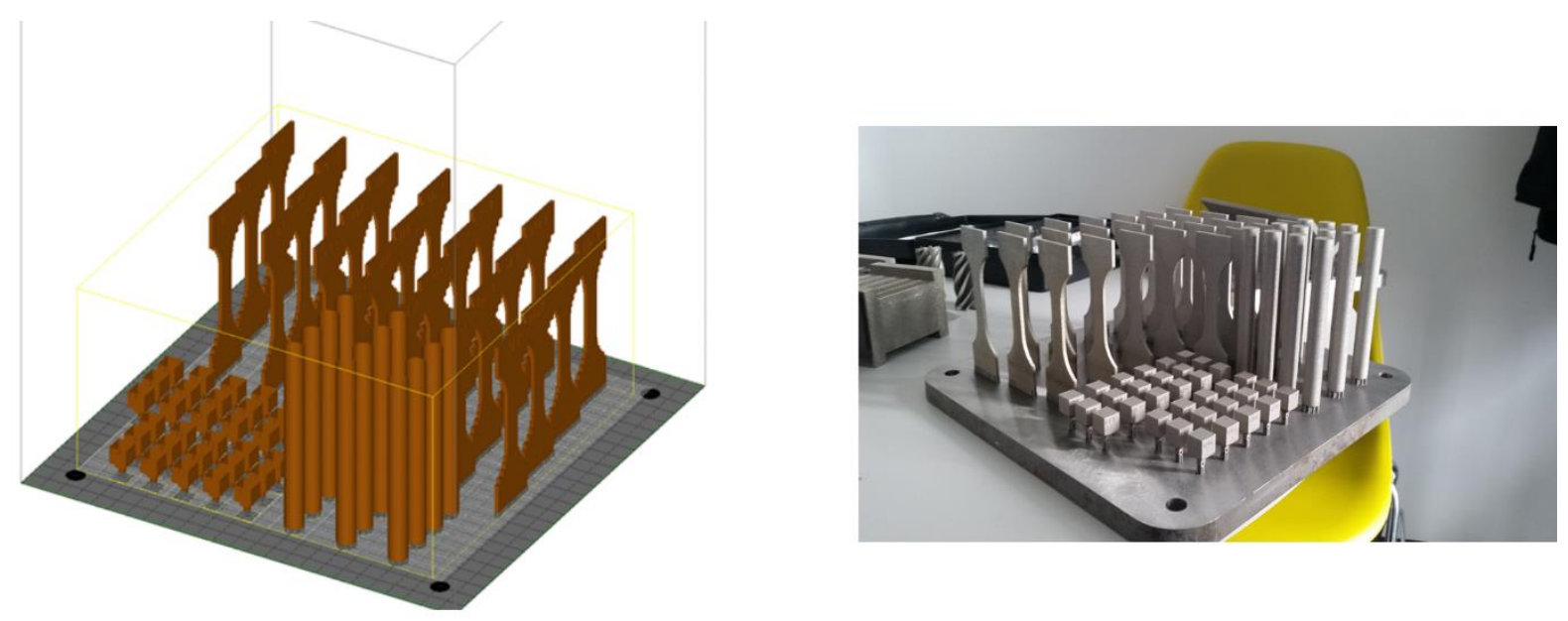

Figure 12. Left) Density cubes, as they appeared in the assembly diagram, Right) the as-built density cube on a base plate. 


\subsubsection{Titanium Ti6Al4V Powder (Experiment E)}

A larger L25 orthogonal array was used in this section to obtain greater accuracy for the optimal input parameters for achieving maximum bulk density. Four parameters, the laser power $(\mathrm{W})$, point distance $(\mu \mathrm{m})$, hatch spacing $(\mu \mathrm{m})$ and exposure time $(\mu \mathrm{s})$, were used to create 25 different combinations using the L25 orthogonal array, as shown in Table 6 . The optimal parameters, as suggested by the manufacturer, were represented in sample A1.

The results from this experiment are presented and discussed in detail in Chapter 5.1.3

\begin{tabular}{|c|c|c|c|c|c|c|}
\hline $\begin{array}{c}\text { Sample } \\
\text { Label }\end{array}$ & $\begin{array}{c}\text { Laser } \\
\text { Power } \\
(\mathbf{W})\end{array}$ & $\begin{array}{c}\text { Point } \\
\mathbf{d i s t a n c e} \\
(\boldsymbol{\mu m})\end{array}$ & $\begin{array}{c}\text { Hatch } \\
\text { Spacing } \\
(\boldsymbol{\mu m})\end{array}$ & $\begin{array}{c}\text { Exposure } \\
\text { Time }(\boldsymbol{\mu s})\end{array}$ & $\begin{array}{c}\text { Energy } \\
\text { Density } \\
\left(\mathbf{J m m}^{-3}\right)\end{array}$ & $\begin{array}{c}\text { Scan } \\
\text { Speed } \\
\left(\mathbf{m m s}^{-1}\right)\end{array}$ \\
\hline A1 & 185 & 50 & 100 & 100 & 74 & 500 \\
\hline A2 & 189 & 50 & 119 & 112 & 71 & 446 \\
\hline A3 & 193 & 50 & 138 & 125 & 70 & 400 \\
\hline A4 & 197 & 50 & 157 & 138 & 69 & 362 \\
\hline A5 & 200 & 50 & 175 & 150 & 69 & 333 \\
\hline A6 & 193 & 62 & 100 & 112 & 70 & 554 \\
\hline A7 & 197 & 62 & 119 & 125 & 67 & 496 \\
\hline A8 & 200 & 62 & 138 & 138 & 65 & 449 \\
\hline A9 & 185 & 62 & 157 & 150 & 57 & 413 \\
\hline A10 & 189 & 62 & 175 & 100 & 35 & 620 \\
\hline A11 & 200 & 75 & 100 & 125 & 67 & 600 \\
\hline A12 & 185 & 75 & 119 & 138 & 57 & 543 \\
\hline A13 & 189 & 75 & 138 & 150 & 55 & 500 \\
\hline A14 & 193 & 75 & 157 & 100 & 33 & 750 \\
\hline A15 & 197 & 75 & 175 & 112 & 34 & 670 \\
\hline A16 & 189 & 88 & 100 & 138 & 59 & 638 \\
\hline A17 & 193 & 88 & 119 & 150 & 55 & 587 \\
\hline A18 & 197 & 88 & 138 & 100 & 32 & 880 \\
\hline A19 & 200 & 88 & 157 & 112 & 32 & 786 \\
\hline A20 & 185 & 88 & 175 & 125 & 30 & 704 \\
\hline A21 & 197 & 100 & 100 & 150 & 59 & 667 \\
\hline A22 & 200 & 100 & 119 & 100 & 34 & 1000 \\
\hline A23 & 185 & 100 & 138 & 112 & 30 & 893 \\
\hline A24 & 189 & 100 & 157 & 125 & 30 & 800 \\
\hline A25 & 193 & 100 & 175 & 138 & 30 & 725 \\
\hline
\end{tabular}

Table 6. Laser parameters used for DOE. 


\subsection{Experiment A - Direct Base Plate Method}

\subsubsection{Objectives}

This section outlines the method used for an experiment carried out in the beginning of the project, which served as a precursor to the research performed later with single track structures. The material used in this experiment was stainless steel 316L. The purpose of the first experiment, known as Experiment $A$, was to investigate the influence of laser power (W) and exposure time ( $\mu \mathrm{s})$ on the formation of square-shaped structures built on top of a separate plain carbon steel plate substrate. The track formation, size and appearance were the features of interest.

\subsubsection{Experimental Design}

The structures were initially designed to include multiple layers, ranging from 2 to 48 layers. The original aim of the experiment was to investigate the change in the physical structure from layer to layer. However, the build failed after only building the first layer, and thus only single layer structures were investigated.

Six sets of processing parameter settings were used, as seen in Table 7. The point distance and hatch spacing were kept constant at $65 \mu \mathrm{m}$ and $124 \mu \mathrm{m}$, respectively. The laser spot diameter was kept constant at $70 \mu \mathrm{m}$. The layer thickness used for this build was $50 \mu \mathrm{m}$ and a stripes-type hatch pattern was used. Since the build failed after only one layer, all structures were built in the horizontal direction, perpendicular to the wiper blade direction.

\begin{tabular}{|c|c|c|}
\hline $\begin{array}{c}\text { Sample } \\
\text { ID }\end{array}$ & $\begin{array}{c}\text { Laser } \\
\text { Power } \\
(\mathrm{W})\end{array}$ & $\begin{array}{c}\text { Exposure } \\
\text { Time } \\
(\mu \mathrm{s})\end{array}$ \\
\hline 1 & 100 & 75 \\
\hline 2 & 100 & 150 \\
\hline 3 & 150 & 75 \\
\hline 4 & 150 & 150 \\
\hline 5 & 200 & 75 \\
\hline 6 & 200 & 150 \\
\hline
\end{tabular}

Table 7. Experiment A (SS316L) - Laser Parameters used. 
A series of 8 square-base shaped 3D objects were to be built for each setting, each with an area of $20 \mathrm{~mm}$ by $20 \mathrm{~mm}$. These squares were assembled into rectangular regions, each measuring $80 \mathrm{~mm}$ by $20 \mathrm{~mm}$. The square structures were designed to have an ascending number of layers to be built, ranging from 2 to 48 . The number of layers to be built for each square-base structure and their position is noted in Figure 13.
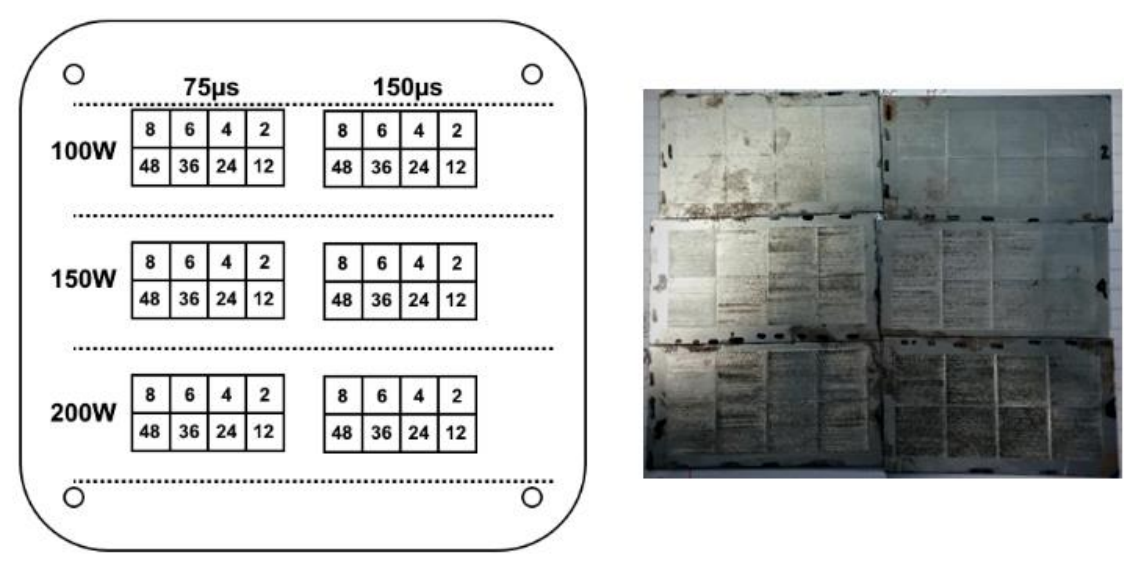

Figure 13. Experiment A (SS316L) - Experimental Design Left) Experimental Design, Right) Photographs of sectioned samples.

Rather than being built directly unto the base plate, a mild steel plate was used, which was $3.8 \mathrm{~mm}$ thick. This plate was machined to fit over the base plate of the Renishaw 250 machine, as well as to bolt onto it. The purpose of this sub-plate was to act in a sacrificial capacity. Once the build was complete, the sub-plate could be removed and divided quite easily, facilitating subsequent microscopy.

After the build was completed, the sub-plate was removed from the base plate and each rectangle was cut into separate pieces. The rectangular pieces were observed under a Keyence VHX 6000 series light microscope, taking photographs of the formed melt pools from an overhead view.

After taking these images, each square structure in the rectangular pieces were cut out. From each square structure, cuts would be taken, one cut in the horizontal direction, that is, perpendicular to the build direction, and one in the vertical direction, parallel to the build direction. The horizontal cuts were used to observe an effective cross-section of a single row 
of melt pools along the laser direction, whilst the longitudinal cuts were used to observe a cross-section across multiple rows perpendicular to the laser path. These samples were mounted, etched and observed under a Reichert-Jung MEF3 light microscope fitted with a digital camera. Greater detail on the metallographic methods used to prepare these samples can be found in Appendix 2. The etchant used was Kalling's Reagent No. 2, which is used to show the general structure of austenitic stainless alloys. The combined cross-sectional and overhead photographs are shown in Figure 20 through Figure 25, with the location of the cut-direction shown below the cross-sectional image using a red arrow.

The resulting photographs taken from the single layer experiment from both the surface and cross-section samples were analysed using ImageJ image processing software to measure the sizes of certain features such as melt pool dimensions, layer thickness and gap sizes between visible structures in the cross-sectional samples. The results from this experiment are presented and discussed in detail in Chapter 5.2

\subsection{Experiment B - Single-Lines on Recessed Plates Method}

\subsubsection{Objectives}

The purpose of this experiment was to investigate the effect of two processing parameters, laser power $(\mathrm{W})$ and scan speed $\left(\mathrm{mms}^{-1}\right)$, on the formation of single line structures. Instead of creating several overlapping tracks like in Experiment $A$, this experiment was to focus on the formation of individual tracks, separated from neighbouring tracks by using a large hatch spacing. The same material powder, stainless steel 316L, would be used for this experiment.

\subsubsection{Experimental Design}

The parameters were divided into six groups, each group with a specific power setting ( $75 \mathrm{~W}$, 100W, 125W, 150W, 175W and 200W). For each group, 10 different laser speeds were used, ranging from $100 \mathrm{mms}^{-1}$ to $1000 \mathrm{mms}^{-1}$, with a $100 \mathrm{mms}^{-1}$ step size. The exposure time was altered whilst the point distance used for every sample was kept constant at $60 \mu \mathrm{m}$. The parameters used can be seen in Table 8. 


\begin{tabular}{|c|c|c|c|c|c|c|c|c|c|c|c|}
\hline & \multicolumn{10}{|c|}{ Scan Speed $\left(\mathrm{mms}^{-1}\right)$} \\
\hline & & 100 & 200 & 300 & 400 & 500 & 600 & 700 & 800 & 900 & 1000 \\
\hline & & \multicolumn{10}{|c|}{ Exposure Time ( $\mu s)$} \\
\hline \multirow{6}{*}{ 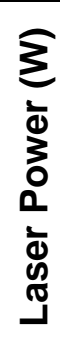 } & 200 & 600 & 300 & 200 & 150 & 120 & 100 & 86 & 75 & 67 & 60 \\
\hline & 175 & 600 & 300 & 200 & 150 & 120 & 100 & 86 & 75 & 67 & 60 \\
\hline & 150 & 600 & 300 & 200 & 150 & 120 & 100 & 86 & 75 & 67 & 60 \\
\hline & 125 & 600 & 300 & 200 & 150 & 120 & 100 & 86 & 75 & 67 & 60 \\
\hline & 100 & 600 & 300 & 200 & 150 & 120 & 100 & 86 & 75 & 67 & 60 \\
\hline & 75 & 600 & 300 & 200 & 150 & 120 & 100 & 86 & 75 & 67 & 60 \\
\hline
\end{tabular}

Table 8. Experiment B (SS316L) - Processing parameters used.

For this experiment, the base plate was modified to be able to fit rectangular shaped mild steel inserts. The base-plate had five rectangular sections milled out from the surface, cutting a rectangular volume measuring $200 \mathrm{~mm}$ by $25 \mathrm{~mm}$. The insert design would have the exact dimensions of the milled sections and would be cut from stainless steel plate of the same thickness as the depth, allowing inserts to fit in easily. Additionally, both surfaces were plain, allowing the insert to be retained by gravity. The design of the milled base plate and inserts can be seen in Figure 14 below. 


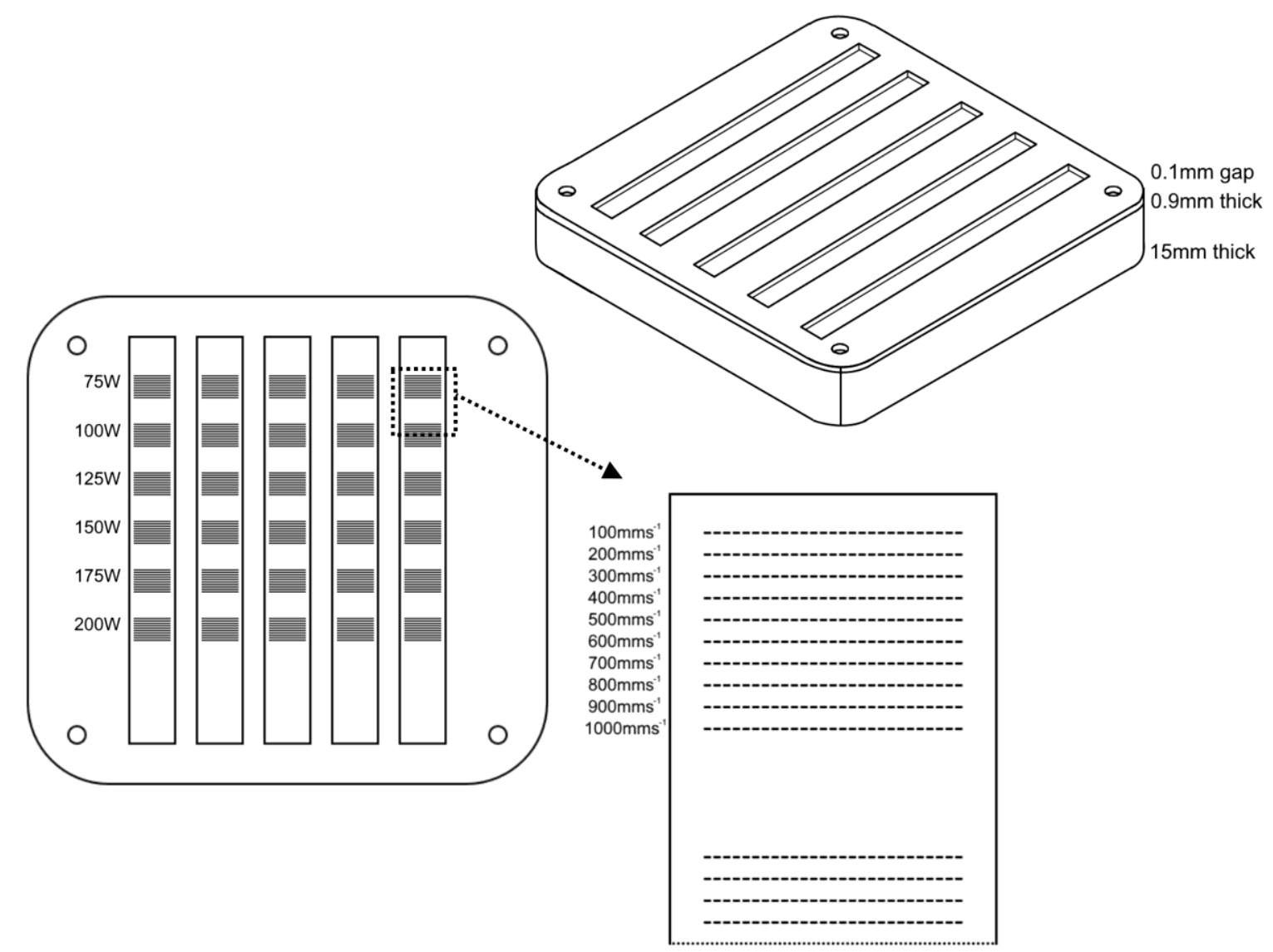

Figure 14. Experiment B (SS316L) - Experimental design.

This design was chosen for several reasons. Firstly, it would be easier and quicker to investigate the final single tracks, unlike the design used for experiment $A$. The design used for experiment $A$ required an entire plate to be bolted onto the base plate, and subsequently that plate had to be machined several times before the structures produced could be isolated and undergo metallographic preparation. Furthermore, an entire steel plate had to be used with only a very limited area being used for the experiment. Using the insert method, the structure would be almost immediately available for metallographic preparation and only a single cut would be needed to obtain the cross-section. Several inserts could be prepared in advance, allowing repetition of the experiment to be performed very easily.

The modified base plate was bolted into a Renishaw AM250 machine. A mild steel insert was placed in each of the five recesses. The machine was placed under vacuum conditions and an argon atmosphere was introduced into the build chamber, just as if a regular build was being prepared. The build plate was lowered by $50 \mu \mathrm{m}$, and powder was deposited from 
the hopper. This amount of powder was spread over the inserts to achieve an estimated $50 \mu \mathrm{m}$ powder layer thickness.

On each insert, six sets of single lines were produced, each with ten lines, as seen in the bottom-right image in Figure 14. Each set had an assigned laser power setting, ranging from $200 \mathrm{~W}$ to $75 \mathrm{~W}$. Each line produced had a different exposure time, changing the scan speed used for each line, ranging from $1000 \mathrm{mms}^{-1}$ to $100 \mathrm{mms}^{-1}$. Effectively the experiment was repeated five times, once for each insert, at different positions on the base plate. After the lines were built, the machine was evacuated, the chamber door could be opened and the inserts were removed from the build plate. The lines were investigated under a ZEISS Smartzoom 5 Automated Digital Microscope to obtain the topographical images, whilst cross-sections were taken using similar methods used in Experiment $A$ and Appendix 2. These cross-sections were observed and recorded under a Zeiss Primotech Light Microscope, and the width and depth of each track was measured from these images. The results from this experiment are presented and discussed in detail in Chapter 5.3

\subsection{Crucible Method (CM)}

\subsubsection{Introduction}

In both experiments $A$ and $B$, the substrates used in each experiment were made from mild steel. Apart from being made from a completely different material than the material powder used, these substrates were made via machining rather than additive manufacturing. The resulting structures from these experiments was indicative of track formation for the very first layers of a build, where the powder is melted unto a base plate. However, these tracks would not be representative of those formed at subsequent layers much further away from the base plate. Therefore, a novel substrate design, called the "crucible", was developed to tackle these issues. The crucible substrate would not only be able to emulate the in-situ surface conditions of additive manufacturing, but could also allow for adjustments in the layer depth to be used as an additional process parameter during the investigation. 


\subsubsection{Crucible Design}

The crucible is a rectangularly shaped substrate, roughly measuring $10 \mathrm{~mm} \times 15 \mathrm{~mm} x$ $5 \mathrm{~mm}$, ass seen in Figure 15. At the top-most, vertical side of the crucible, that is, opposite the side of the base plate it is built on, is a rectangular recess that is only a few layers deep, or even a single layer. For the experiments performed in this research, the layer thickness for an individual layer in each build was $50 \mu \mathrm{m}$, whilst the recess depth ranged from 50 $200 \mu \mathrm{m}$. The purpose of this recess is to hold an isolated layer of powder of a depth controlled by the user, and thus can be introduced as an input parameter. The crucible allows for the investigation of single tracks to be built on the same type of material as the tracks themselves, avoiding the use of possibly dissimilar materials, or materials made using different processes.
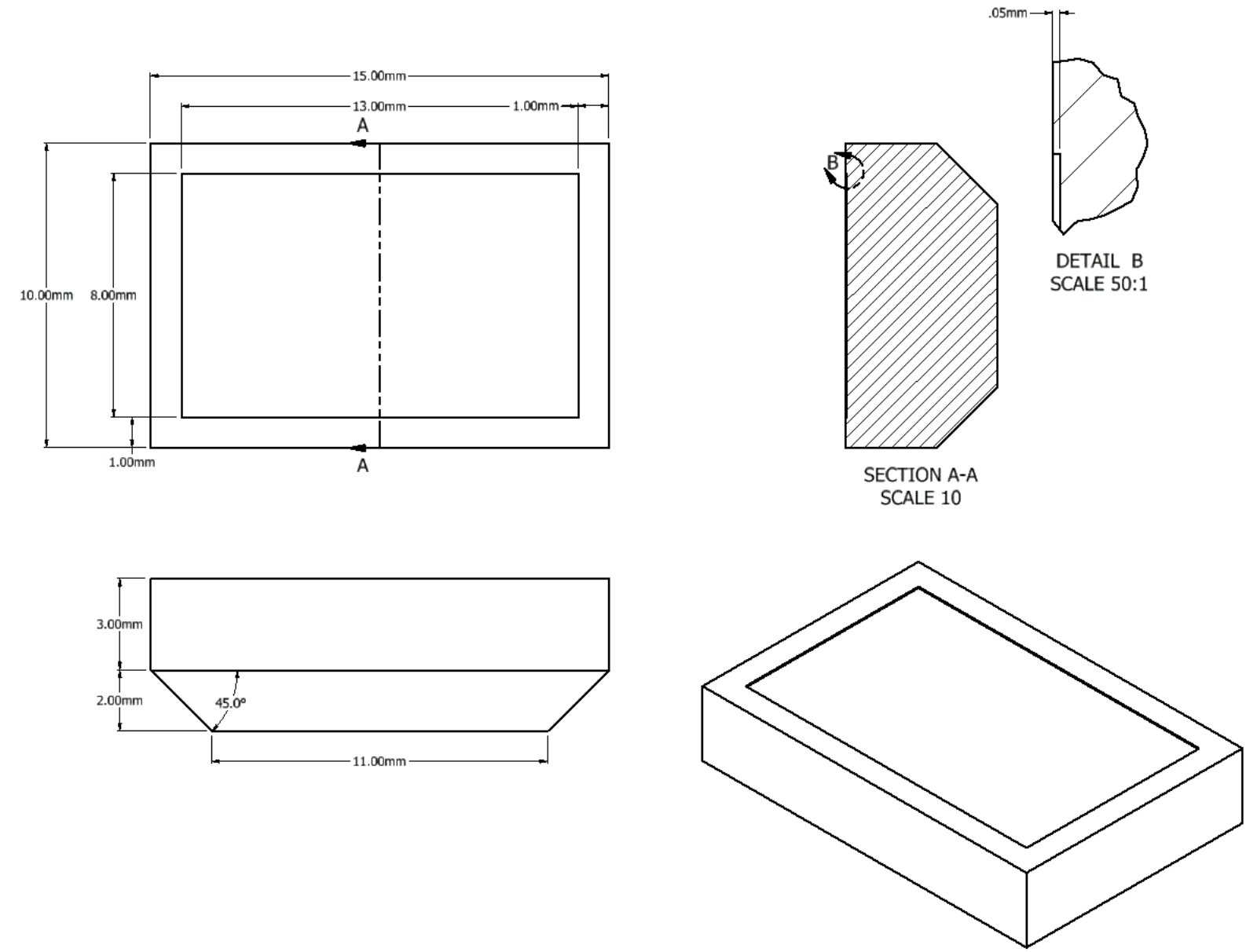

Figure 15. The Crucible Design.

Upper Left: Vertical view, Lower Left: Horizontal view, Upper Right: Cross Section showing recess, Lower Right: Diagonal view. 
The general crucible structure would be built using standard operating parameters for the material being used, whilst the single tracks being investigated are built using different combinations of testing parameters. Between the construction of the crucible and the construction of the tracks, which is the final layer of the build, the AM250 was paused for 15 minutes. This was done in order to allow the previous layers to undergo sufficient cooling, thus the heat generated from the construction of the crucible was minimised in order for it to not be a factor in the experiment.

Each crucible holds a set of tracks, which ranged from 3 to 12, which are each separated from one another by a minimum of $500 \mu \mathrm{m}$. An example of a 3-track crucible can be seen on the right-hand side of Figure 16. The experiment is constructed in one build, with no intermediate steps to follow during, before or after a build like in previous experimental methods proposed. The crucibles are removed from the base plate by the use of a set of pliers, or even by hand. Several crucibles can be placed on a base plate, allowing for repetitions or a very wide parameter selection range. On the AM250, around 90-120 crucibles could easily be built on the $250 \mathrm{~mm} \times 250 \mathrm{~mm}$ build envelope, as seen on the lefthand side of Figure 16.
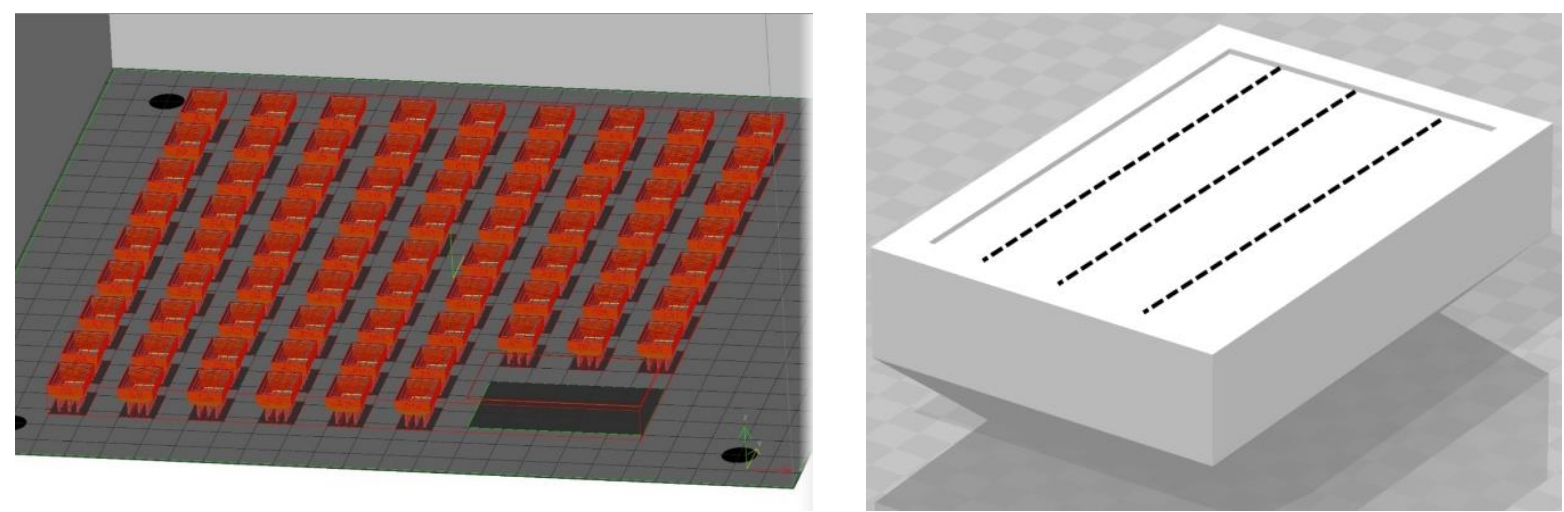

Figure 16. Left) CAD drawing of crucibles used during an experiment, Right) Three single track structures placed at the top of the crucibles.

The experimental design for each crucible experiment was similar, though there were crucial differences between each one. For example, whilst Experiments $C$ and $E$ introduced the 
layer thickness as a parameter, tracks made for Experiment D used only a single layer. These details are discussed in greater detail below.

\subsubsection{Experiment C - Verification of Single-Track Crucible Methodology}

\section{Objectives}

This experiment sought to utilise the standardised crucible design mentioned in the previous section to its fullest ability on a new experiment. It was the first experiment to use the methodology described in the previous section. Stainless steel 316L was used as the material powder.

The main objective of this experiments was to investigate the influence of laser power, scan speed, and surface energy density on the formation of single-track structures. Surface energy density is a value calculated from the laser power and the scan speed used to indicate the amount of energy being delivered from the laser to the area under its influence. It is calculated using the following equation.

$$
E \rho=\frac{P}{v d}
$$

where $E \rho$ is surface energy density $\left(\mathrm{Jmm}^{-2}\right), P$ is laser power $(\mathrm{W}), v$ is scan speed $(\mathrm{mm} / \mathrm{s})$ and $d$ is laser spot diameter $(\mathrm{mm})$. The variance of laser power and scan speed for fixed surface energy density values was investigated. Additionally, due to the new crucible methodology, these different parameter combinations were investigated at varying layer powder depths by introducing crucibles with varying recess depths.

\section{Experimental Design}

7 surface energy density values were established, ranging from $80 \mathrm{~J} \mathrm{~mm}^{-2}$ to $4 \mathrm{~J} \mathrm{~mm}^{-2}$. For each energy density value, 4 laser power settings would be used, ranging for $200 \mathrm{~W}$ to $100 \mathrm{~W}$. The scan speed for each corresponding laser power setting was calculated according to the following equation, which is a slight modification of equation above.

$$
v=\frac{P}{E \rho \cdot d}
$$


Where $d$ was kept constant at $70 \mu \mathrm{m}$. A table of the different parameter combinations can be seen in Table 9 below.

\begin{tabular}{|c|c|c|c|c|c|c|c|c|}
\hline & \multicolumn{7}{|c|}{ Surface Energy Density $\left(\mathrm{Jmm}^{-2}\right)$} \\
\hline & & 80 & 40 & 30 & 20 & 12 & 8 & 4 \\
\hline & & \multicolumn{7}{|c|}{ Scan Speed $\left(\mathrm{mms}^{-1}\right)$} \\
\hline \multirow{4}{*}{ 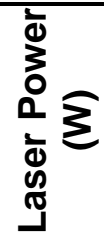 } & 200 & 65 & 130 & 173 & 260 & 433 & 650 & 1299 \\
\hline & 160 & 52 & 104 & 139 & 208 & 346 & 520 & 1039 \\
\hline & 130 & 42 & 84 & 113 & 169 & 281 & 422 & 844 \\
\hline & 100 & 32 & 65 & 87 & 130 & 217 & 325 & 650 \\
\hline
\end{tabular}

Table 9. Experiment C (SS316L) - Processing parameters.

For each parameter, each parameter combination would have 10 single tracks, each $15 \mathrm{~mm}$ in length, built on 4 crucible substrates, each one with a different recess depth. The recess depth ranged from $50 \mu \mathrm{m}$ to $200 \mu \mathrm{m}$ with a step size of $50 \mu \mathrm{m}$. The crucibles were built at standard parameters for stainless steel 316L. In total, 112 crucibles with single track structures were built during this experiment. After the build was completed using the Renishaw AM250, the samples were removed and collected off the base plate and labelled accordingly. Each crucible had the topmost surface photographed using a ZEISS Smartzoom 5 Automated Digital Microscope. These images would be used to examine the overall stability of the lines formed.

Crucible samples with successfully built lines were prepared for metallographic examination. The techniques and specific methods used are listed in Appendices 3 and 4. Beraha II was used as a new etchant for the metallographic preparation of stainless steel $316 \mathrm{~L}$ samples. At a colour etchant, it was found to clearly distinguish grains and grain boundaries much move vividly than the previous used Kalling's Reagent.

The samples were examined under a Zeiss Primotech Light Microscope. Cross-sectional images of the single melt tracks and any notable features around the melt were photographed. The image processing package Fiji, [105], was used to measure the dimensions of each track photograph. 
This included:

a. Maximum width, the measurement of the widest section of the melt.

b. Base width, the width of the track at the surface level of the substrate.

c. Height, the height of the track directly above the surface level of the substrate.

d. Remelting depth, the depth of the track directly below the surface level of the substrate.

The size and presence of pores and other notable features was also recorded. The results from this experiment are presented and discussed in detail in Chapter 0

\subsubsection{Experiment D-Single-Tracks on Crucible Substrates}

\section{Objectives}

The purpose of this experiment was to repeat the experimental procedures used in

Experiment B, where single track structures were constructed using stainless steel $316 \mathrm{~L}$

powder onto mild steel inserts. Instead of inserts made of mild steel, the crucible design from

Experiment $\mathrm{C}$ was used as a substrate. Using the crucible design was considered to be

more representative of the laser-powder bed fusion process for layers being built away from the base plate, that is, the bulk of the build.

Building at the level or very near to the baseplate poses major differences. The temperature gradient is much larger, due to the cooling effect of the base plate being so immediate to the track formation. The surface of the base plate is much smoother, as it is a machine polished surface. In contrast, during the bulk of the build, tracks form at greater distances to the base plate, up to around $300 \mathrm{~mm}$ in the Renishaw AM250, relieving an amount of heat lost through conduction to the baseplate. The type of surface that most of the tracks would form on are previously built layers, made of several tracks assembled in some form of stripe formation. This gives a wavy, uneven and much rougher surface when compared to the plane build plate surface. The relative sensitivity of the two approaches deserves attention, and the investigation performed in this section serves to investigate the differences and possible similarities between the two procedures. 


\section{Experimental Design}

The parameters used in this experiment were the exact ones used in Experiment $B$, in which a series of single line tracks were built using stainless steel $316 \mathrm{~L}$ powder atop mild carbon steel inserts. The parameters used can be seen in Table 16 below.

\begin{tabular}{|c|c|c|c|c|c|c|c|c|c|c|c|}
\hline & \multicolumn{10}{|c|}{ Scan Speed $\left(\mathrm{mms}^{-1}\right)$} \\
\hline & & $\overline{0}$ & 200 & 300 & 400 & 500 & 600 & 700 & 800 & 900 & 1000 \\
\hline & & \multicolumn{10}{|c|}{ Exposure Time $(\mu \mathrm{s})$} \\
\hline \multirow{6}{*}{ 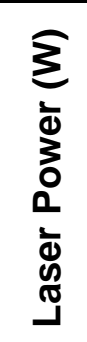 } & 10 & 600 & 300 & 200 & 150 & 120 & 100 & 86 & 75 & 67 & 60 \\
\hline & 175 & 600 & 300 & 200 & 150 & 120 & 100 & 86 & 75 & 67 & 60 \\
\hline & 150 & 600 & 300 & 200 & 150 & 120 & 100 & 86 & 75 & 67 & 60 \\
\hline & 125 & 600 & 300 & 200 & 150 & 120 & 100 & 86 & 75 & 67 & 60 \\
\hline & 100 & 600 & 300 & 200 & 150 & 120 & 100 & 86 & 75 & 67 & 60 \\
\hline & $\overline{75}$ & 600 & 300 & 200 & 150 & 120 & 100 & 86 & 75 & 67 & 60 \\
\hline
\end{tabular}

Table 10. Experiment D (SS316L) - Processing parameters used.

For each parameter listed, a set of three single track melts were produced within the recess depth of a crucible. Since the thickness of the powder layer used for Experiment B was estimated at $50 \mu \mathrm{m}$, the crucible pit depth was set at $50 \mu \mathrm{m}$.

After being built on the Renishaw AM250, the exact metallographic preparation procedures used for Experiment $\mathrm{C}$ were performed on the samples collected in this experiment. The results from this experiment are presented and discussed in detail in Chapter 0

\subsubsection{Experiment E-Crucible Single-Track experiments using Ti-6Al-4V}

\section{Objectives}

In this section, the crucible substrate design was used to create single-line tracks using the titanium alloy Ti-6Al-4V metal powder. Apart from investigating the effect of laser power and scan speed on the formation of Ti-6AL-4V single-tracks, the effect of the powder depth was also investigated by varying the recess depth of the crucible substrate, similar to experiment 
C. Additionally, this experiment was performed to test the applicability of the crucible methodology using a different powder material to stainless steel 316L.

\section{Experimental Design}

To accomplish the experimental objective, three groups of parameters were formed, each with a specific laser power used (100W, 150W and 200W). For each group, six different scan speeds were to be used, which would be varied by altering the point distance and exposure times. The array of parameter combinations used for this experiment are shown in Table 11 below.

\begin{tabular}{|c|c|c|c|c|}
\hline $\begin{array}{c}\text { Sample } \\
\text { ID }\end{array}$ & $\begin{array}{c}\text { Power } \\
(\mathrm{W})\end{array}$ & $\begin{array}{c}\text { Point } \\
\text { Distance } \\
(\mu \mathrm{m})\end{array}$ & $\begin{array}{c}\text { Exposure } \\
\text { Time } \\
(\mu \mathrm{s})\end{array}$ & $\begin{array}{c}\text { Scan } \\
\text { Speed } \\
\left(\mathrm{mms}^{-1}\right)\end{array}$ \\
\hline A1 & 100 & 50 & 100 & 500 \\
\hline A2 & 100 & 60 & 80 & 750 \\
\hline A3 & 100 & 75 & 75 & 1000 \\
\hline A4 & 100 & 120 & 80 & 1500 \\
\hline A5 & 100 & 150 & 75 & 2000 \\
\hline A6 & 100 & 180 & 60 & 3000 \\
\hline B1 & 150 & 50 & 100 & 500 \\
\hline B2 & 150 & 60 & 80 & 750 \\
\hline B3 & 150 & 75 & 75 & 1000 \\
\hline B4 & 150 & 120 & 80 & 1500 \\
\hline B5 & 150 & 150 & 75 & 2000 \\
\hline B6 & 150 & 180 & 60 & 3000 \\
\hline C1 & 200 & 50 & 100 & 500 \\
\hline C2 & 200 & 60 & 80 & 750 \\
\hline C3 & 200 & 75 & 75 & 1000 \\
\hline C4 & 200 & 120 & 80 & 1500 \\
\hline C5 & 200 & 150 & 75 & 2000 \\
\hline C6 & 200 & 180 & 60 & 3000 \\
\hline
\end{tabular}

Table 11. Experiment E (Ti-6Al-4V) - Processing parameters used.

For each parameter combination, single tracks would be built at increasing layer depths as performed in experiment $\mathrm{C}$. The recess depth of the crucible would increase from $50 \mu \mathrm{m}$ to $200 \mu \mathrm{m}$ with a step size of $50 \mu \mathrm{m}$. For each parameter, a series of 15 single tracks of lengths of $13 \mathrm{~mm}$ would be built atop each of the four crucibles. The crucibles were built using the 
processing parameters recommended by the manufacturers, which were a laser power of $185 \mathrm{~W}$, a point distance of $62 \mu \mathrm{m}$, a hatch spacing of $157 \mu \mathrm{m}$ and an exposure time of $150 \mu \mathrm{s}$. After the build was completed, the samples were removed and collected off the base plate. The tracks were investigated using an Alicona Infinite Focus microscope, which could take both a 2D high resolution image and a 3D surface map of the single track structures From the 3D image, the average height of the tracks, the roughness of the tracks and the roughness of the surfaces surrounding the tracks was measured. These measurements were obtained by taking a profile measurement of the track through its centre, in a direction parallel to the build direction. The average height and line build percentage of each track was calculated from the numerical data from obtained from the profile measurement.

Crucible samples with successfully built lines were prepared for metallographic examination. The techniques and specific methods used are listed in Appendix 5. Kroll's reagent was used as an etchant. The etched samples were examined under a Keyence VHX 6000 series light microscope and cross-sectional images were obtained. The results from this experiment are presented and discussed in detail in Chapter 5.6 


\section{Chapter 5 Results}

\subsection{Optimal Density Parameters}

The following section shows the results from Chapter 4.1 The optimal parameters, which give the highest bulk density values, were identified and used as a comparative value to the results found in experiments $A$ to $E$.

\subsubsection{Stainless Steel 316L Powder (Experiments $A$ and B)}

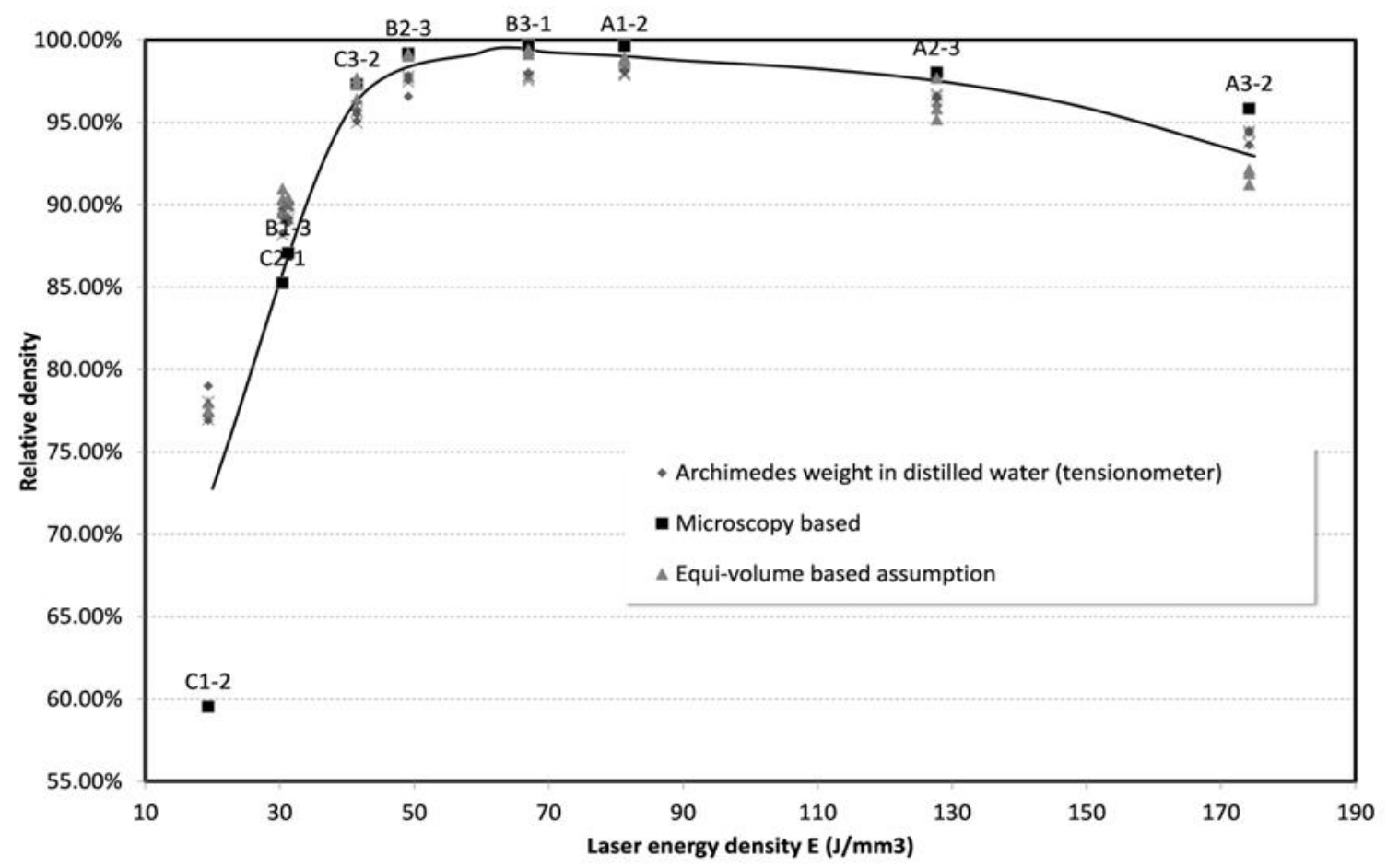

Figure 17. Relative density with laser input energy as measured using gravimetric based methods in Lavery et al. , [104].

The bulk density values for each density measurement value were plot in a graph against the laser energy density values, as seen in Figure 17. The peak density from this experiment was achieved at the sample labelled B3-1. The combination of parameters used at B3-1 was taken as a reference point for which builds using this batch of stainless steel 316 powder could be optimised, in this case in terms of relative density. These parameters combinations 
used to create these samples were a laser power of $180 \mathrm{~W}$, a scan speed of $433 \mathrm{mms}^{-1}$ and an energy density value of $65 \mathrm{Jmm}^{-2}$. These parameter settings were compared to the results obtained at similar parameter settings used in experiments $A$ and $B$.

Samples A1-2, A2-3 and A3-2 were found to have increased porosity as the laser energy density values increased. Decreasing the point distance caused scan speeds to become slower, thereby allowing the laser to deliver more energy into the powder bed per unit of time. This additional energy input caused defects and pores to form, thereby lowering the relative density of the cubes.

\subsubsection{Stainless Steel 316L Powder (Experiments $C$ and D)}

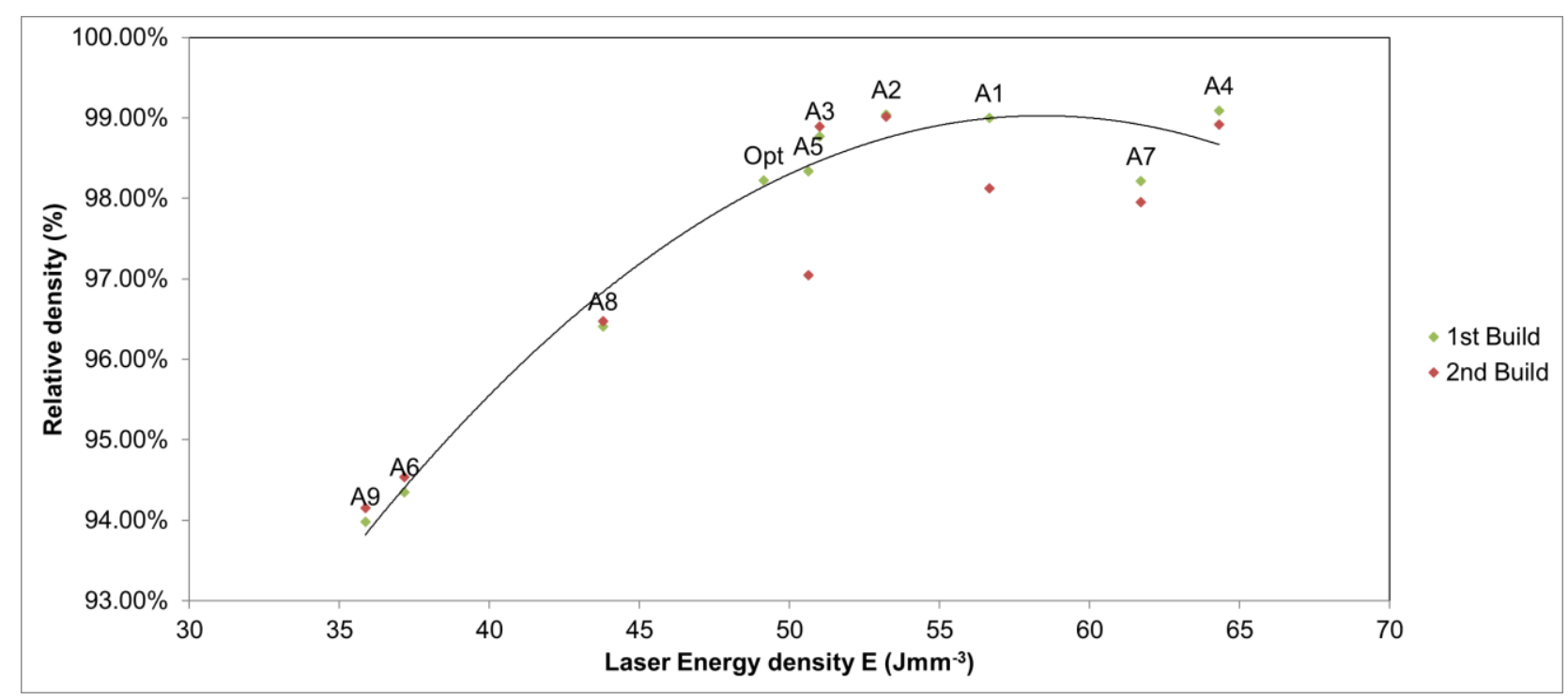

Figure 18. Relative density with input energy measured in DOE experiment for Chapter 5, (SS316L).

The measured density values were used to plot the average relative density of the cubes against the laser energy density used to fabricate them, as seen in Figure 18. It can be seen that the nominal settings, labelled "Opt", did not give the highest relative density for lowest energy density. The optimal parameters for relative density were found at $A 3$, where the laser power was $190 \mathrm{~W}$ and scan speed of $500 \mathrm{mms}^{-1}$, with an energy density value of $64 \mathrm{Jmm}^{-3}$. These values were found to be not at all dissimilar to those obtained for the previous batch, where the optimal energy density value was $65 \mathrm{Jmm}^{-3}$. This indicated that 
there was very little to no changes in the processability of the two batches of material powder.

The combination of parameters used at A3 were taken as a reference point for which builds could be optimised, at least in the case of relative density. These parameters would be compared to the results obtained at similar parameter settings used in experiments $C$ and $D$.

\subsubsection{Titanium Ti6Al4V Powder (Experiment E)}

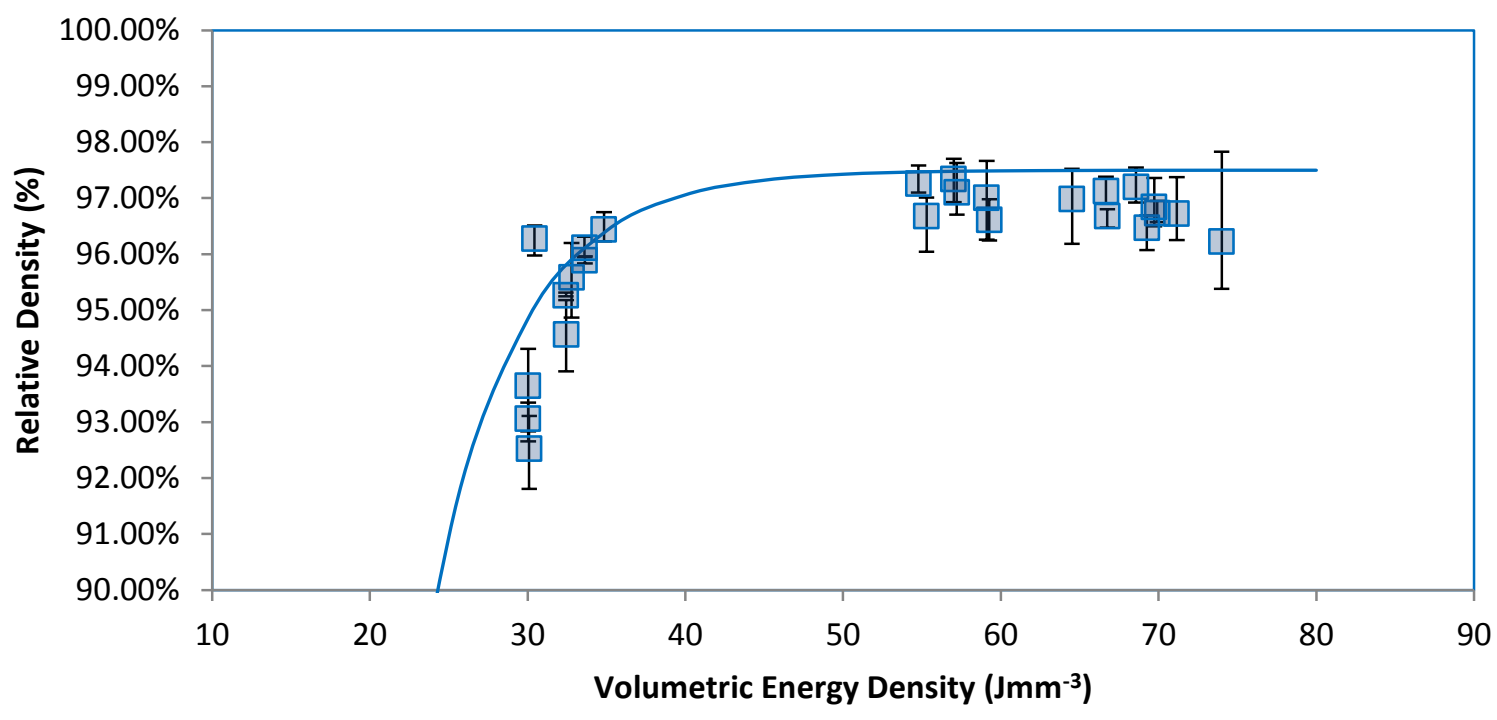

Figure 19. Relative density with input energy measured in DOE experiment for Chapter 6, (Ti-6Al-4V).

The average densities of each sample were plot as a function of laser energy density, as seen in Figure 19. The optimal combination of parameters which gave the highest relative density $(97.3 \%)$ for the lowest possible energy density $\left(57 \mathrm{Jmm}^{-3}\right)$ was found at sample A9. The sample used a laser power of $185 \mathrm{~W}$, a scan speed of $413 \mathrm{mms}^{-1}$, and an energy density value of $57 \mathrm{Jmm}^{-3}$ The nominal parameters, as used for sample A1, was found with an inferior average relative density of $96.2 \%$ at a higher energy density of $74 \mathrm{Jmm}^{-3}$. The scan speed for the nominal parameters was slightly higher, at $500 \mathrm{mms}^{-1}$.

The combination of parameters used at $A 9$ were taken as a reference point for which builds using the current batch of Ti-6Al-4V powder could be optimised, at least in the case of 
relative density. These parameters would be compared to the results obtained at similar parameter settings used in experiment $\mathrm{E}$.

\subsection{Experiment A- Direct Base Plate Method}

\subsubsection{Results}

Table 12 shows the average length, measured in the horizontal direction, and width, measured in the vertical direction, and the average height, measured in both directions, of each stainless steel $316 \mathrm{~L}$ structure. These results were gathered from the overhead images taken using the Keyence VHX 6000 series light microscope, displayed in Figure 20 to Figure 25. The red arrows in the bottom two topographical images of each figure show the direction in which the above cross-sectional image was taken. If applicable, the gap size between successive melt tracks was recorded. In samples 4, 5 and 6, the degree of melt pool overlap was high enough to eliminate any gap formation.

\begin{tabular}{|c|c|c|c|c|c|c|c|}
\hline $\begin{array}{c}\text { Sample } \\
\text { ID }\end{array}$ & $\begin{array}{l}\text { Laser } \\
\text { Exposure } \\
\text { Time } \\
(\mu s)\end{array}$ & $\begin{array}{c}\text { Laser } \\
\text { Power } \\
\text { Output } \\
\text { (W) }\end{array}$ & $\begin{array}{c}\text { Average } \\
\text { Height }(\mu \mathrm{m})\end{array}$ & $\begin{array}{l}\text { Average } \\
\text { Length } \\
(\mu \mathrm{m})\end{array}$ & $\begin{array}{c}\text { Average } \\
\text { Width } \\
(\mu \mathrm{m})\end{array}$ & $\begin{array}{l}\text { Average } \\
\text { Maximum } \\
\text { Gap size } \\
(\mu \mathrm{m})\end{array}$ & $\begin{array}{c}\text { Average } \\
\text { Minimum } \\
\text { Gap size } \\
\quad(\mu \mathrm{m})\end{array}$ \\
\hline 1 & \multirow{3}{*}{$75 \mu \mathrm{s}$} & $100 \mathrm{~W}$ & 16.9 & 70.1 & 83.9 & 65.1 & 28.7 \\
\hline 3 & & $150 \mathrm{~W}$ & 24 & 94.2 & 105.5 & 38.4 & 20.8 \\
\hline 5 & & $200 W$ & 78.15 & 122.6 & 132.5 & $n / a$ & $n / a$ \\
\hline 2 & \multirow{3}{*}{$150 \mu \mathrm{s}$} & $100 W$ & 18.95 & 91.7 & 101.7 & 39.4 & 23 \\
\hline 4 & & $150 \mathrm{~W}$ & 34.3 & 108.2 & 123.6 & $n / a$ & $n / a$ \\
\hline 6 & & $200 W$ & 50.65 & $\mathrm{n} / \mathrm{a}$ & 133.6 & $\mathrm{n} / \mathrm{a}$ & $n / a$ \\
\hline
\end{tabular}

Table 12. Experiment A (SS316L) - Average Length, Width, Height and Gap Size taken from surface images. 


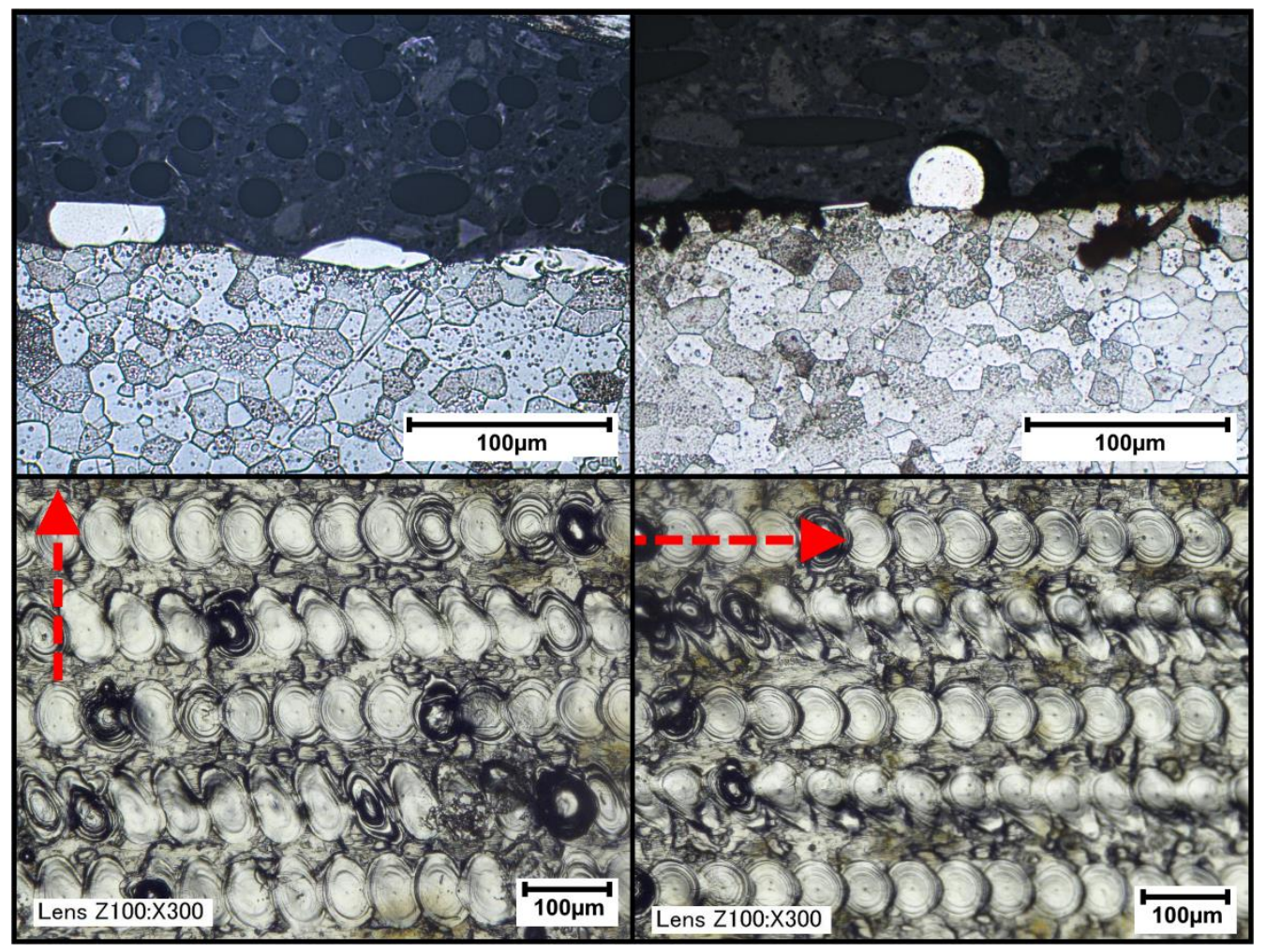

Figure 20. Experiment A (SS316L) - Cross-sectional and topographical results, Sample 1.

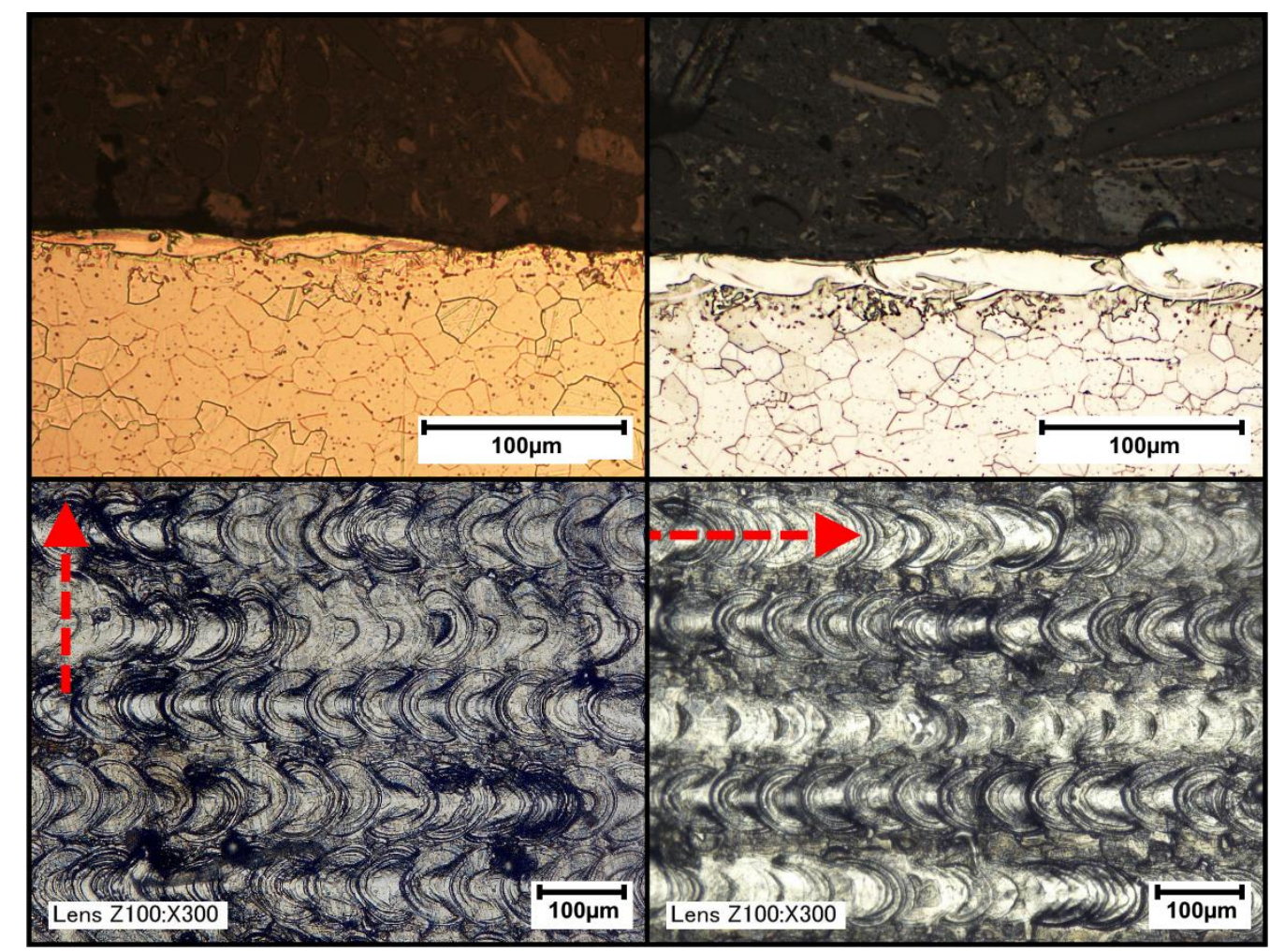

Figure 21. Experiment A (SS316L) - Cross-sectional and topographical results, Sample 2. 


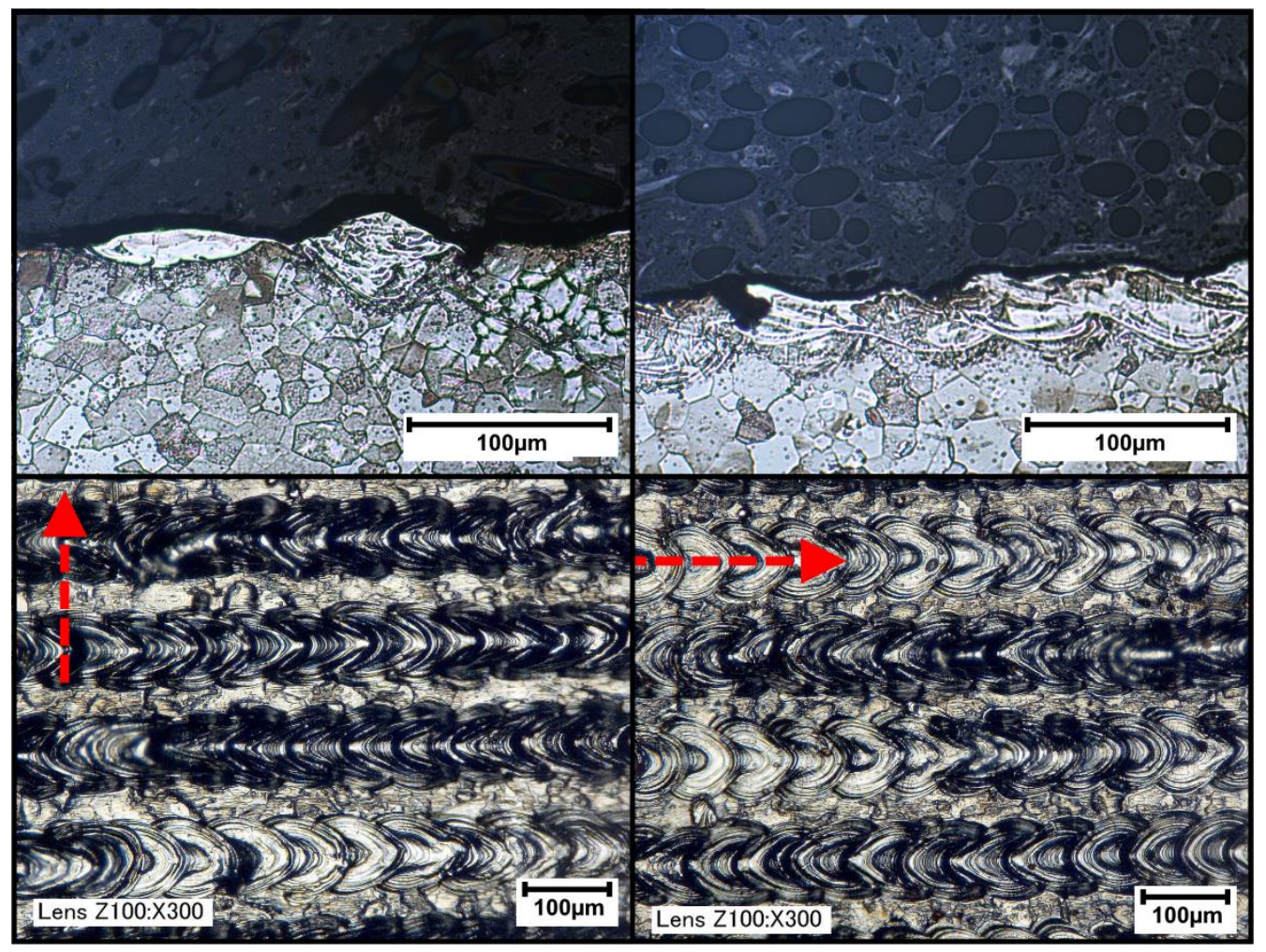

Figure 22. Experiment A (SS316L) - Cross-sectional and topographical results, Sample 3.

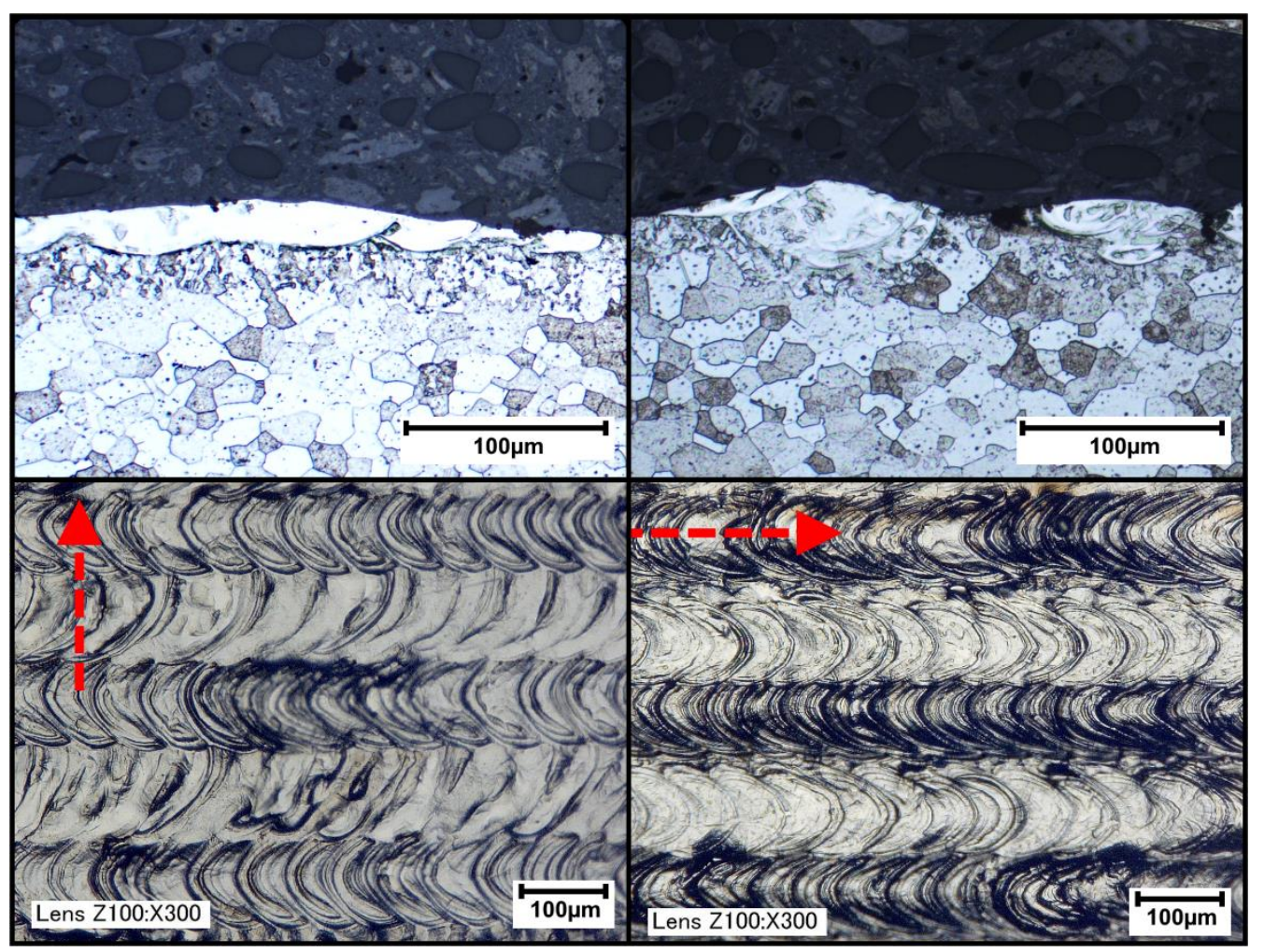

Figure 23. Experiment A (SS316L) - Cross-sectional and topographical results, Sample 4. 


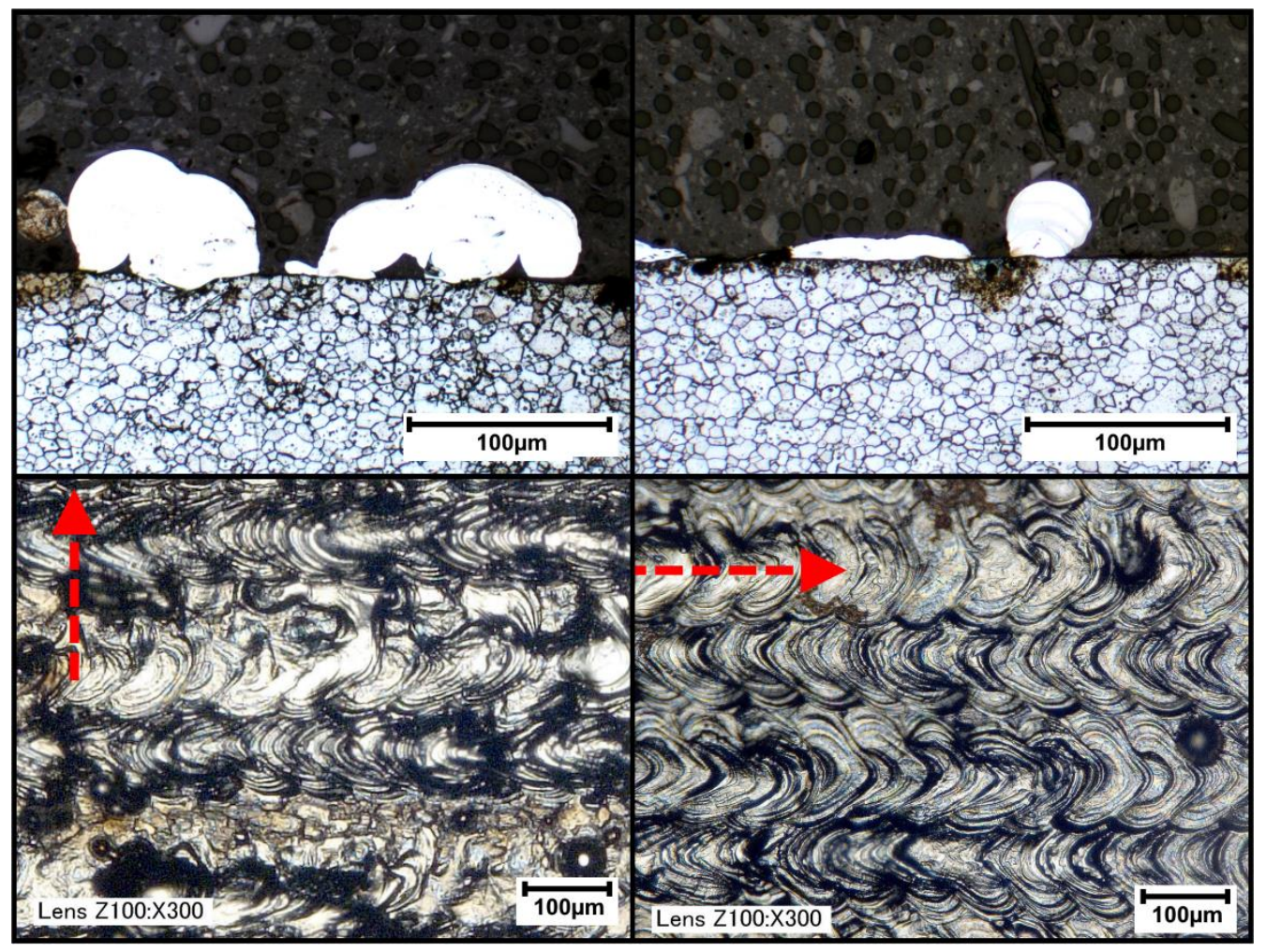

Figure 24. Experiment A (SS316L) - Cross-sectional and topographical results, Sample 5.

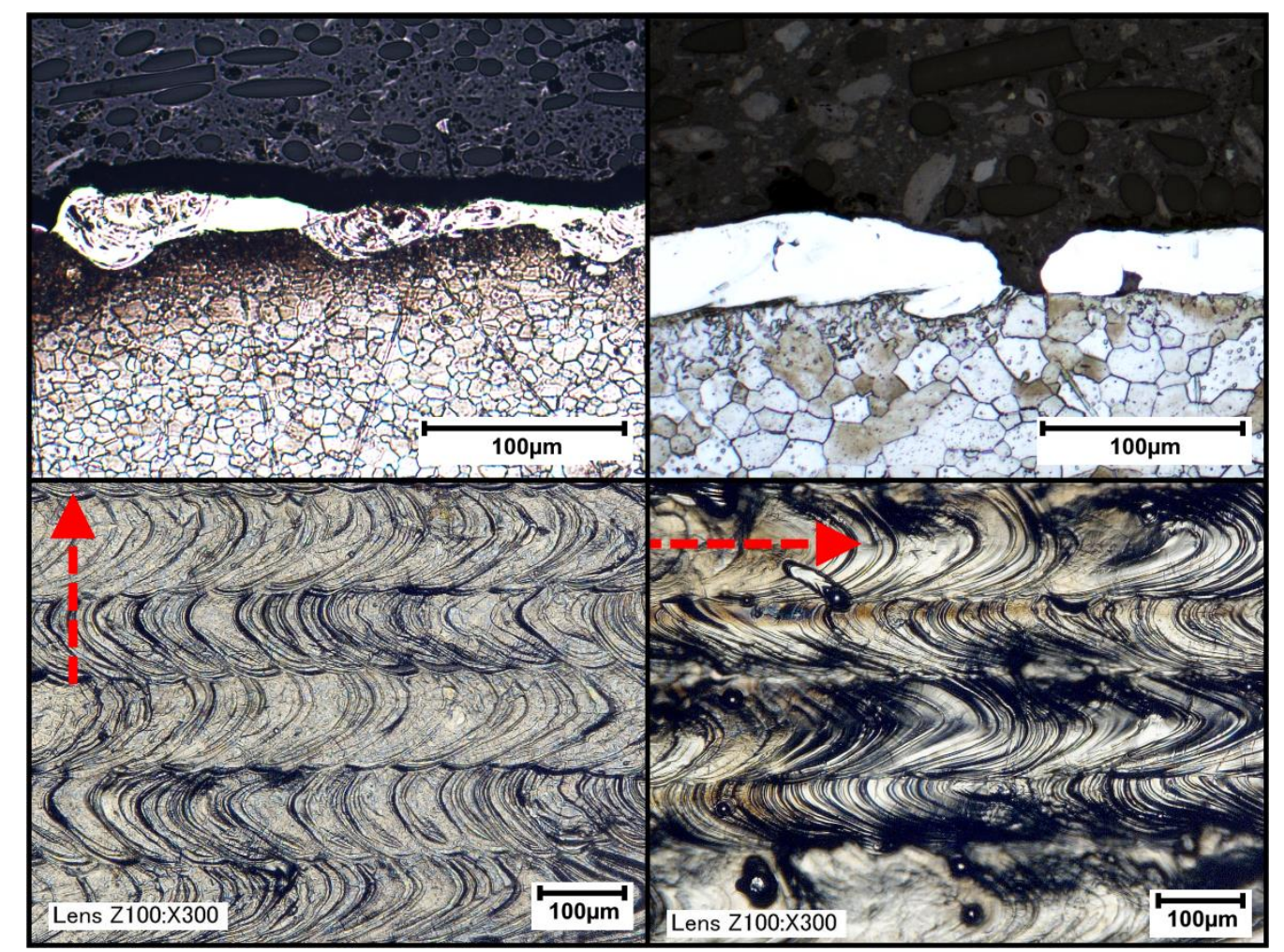

Figure 25. Experiment A (SS316L) - Cross-sectional and topographical results, Sample 6. 
The average length, width and height of the tracks formed during the experiment were plot against the laser power and scan speed used to fabricate them, as seen in Figure 26. Track length, width and height would clearly increase with laser power. It should be noted that the track length value at $200 \mathrm{~W}$ and $150 \mu$ s could not be included as the distinction between separate tracks could not be found due to overlapping of melt pools. Increasing exposure time would also increase these attributes. Since the range in exposure times was limited to two, fairly low values, this increase was not as pronounced as the changes seen between tracks built at different laser powers.

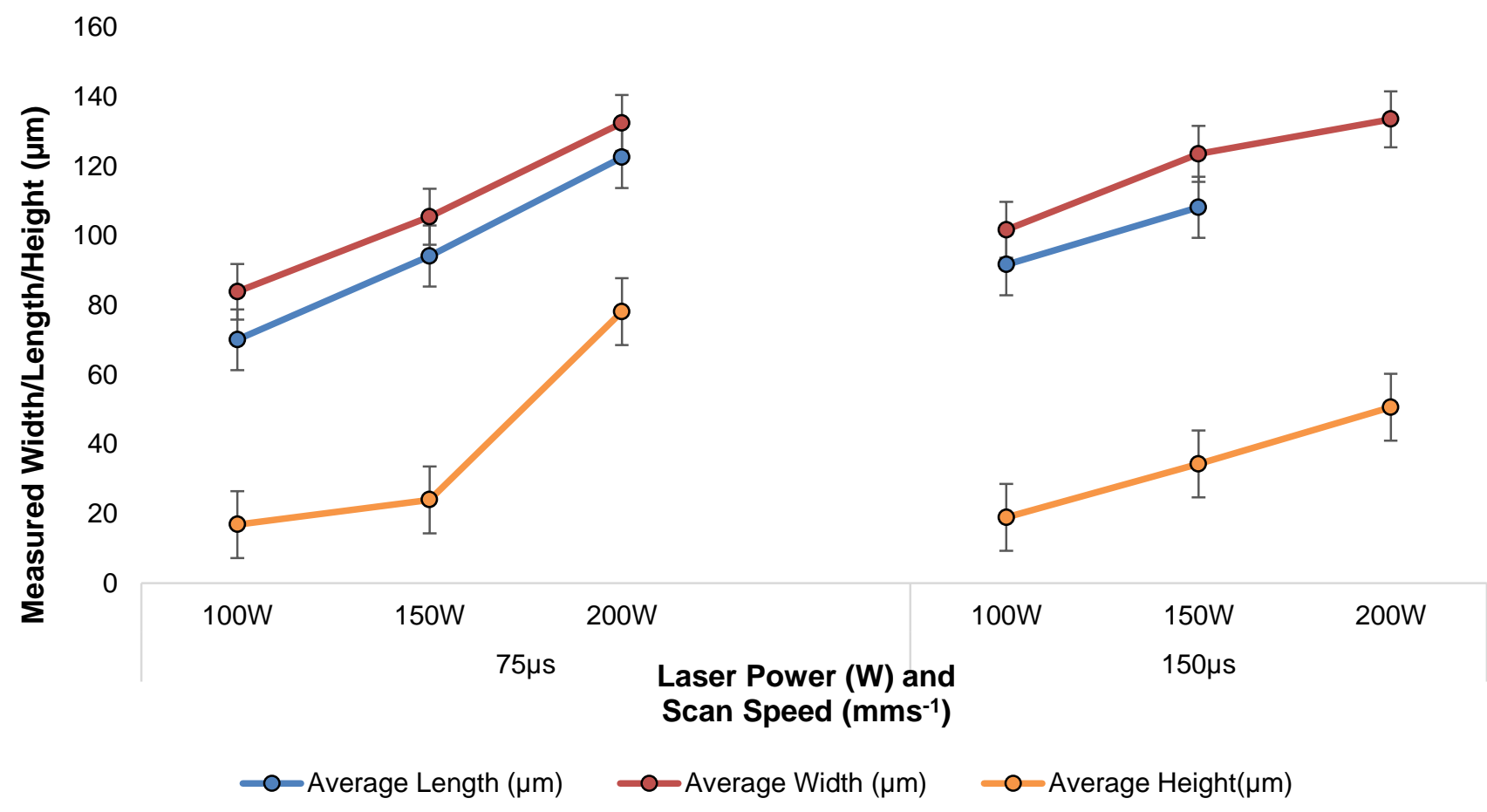

Figure 26. Experiment A (SS316L) - Changes in the track dimensions and gap sizes.

In tracks made using low laser power and low exposure time (samples 1 to 3 ), individual melt beads are easily discernible from one another. A sizeable gap between each successive row of tracks could be observed, as seen in Figure 20, Figure 21 and Figure 22. As laser power was increased, this gap grew smaller until the presence of overlapping tracks prevented gap formation. At high laser power and exposure time (samples 4 to 6 ), it became 
increasingly difficult to isolate individual melt beads, as track overlap had drastically increased, as seen in Figure 23, Figure 24 and Figure 25.

\subsubsection{Discussion}

A sharp demarcation can be seen between the stainless-steel powder structures. The stainless-steel structure is plainly white and without visible grain boundaries, whilst the substrate is clearly stained and has marked grain boundaries. It is possible that the etchant used was not strong enough to affect the grain boundaries in the stainless-steel structure, as it has a higher resistance to chemical attacks.

The height and length values would increase with both laser power and exposure times. The largest structure sizes would be found at 200W. It was shown that increasing the input energy from the laser, whether it is done by increasing the laser power itself or increasing the amount of time the laser spot spent at each point, would cause the volume of melted powder material to increase. The molten material would solidify after the laser had passed to the next point, where heat would be lost through the mechanisms described in Chapter 3. Increasing the volume of molten material would result in increasing the volume of the solidified tracks.

At lower exposure times, particularly for samples 1,3 and 5, made using $75 \mu$ s, the resulting structures solidified with limited wetting and spreading on the substrate. This can be seen in figures 22, 24 and 26, where there was little to no penetration into the substrate. Compared to the other structures formed at the higher exposure time of $150 \mu \mathrm{s}$, as seen in in figure 23 , 25 and 27, the former structures are more spherical, and have a steeper angle coming off of the substrate. Structures made in samples 2, 4 and 6, on the other hand, are flattened and are in complete contact with the substrate. Overall, wetting and spreading of the molten powder is seen to increase with energy input.

\subsubsection{Conclusions}

From the results, it can be concluded that there is systematic variation in track morphology when the process parameters are altered, namely that increasing the input energy tends to 
increase the size of the resultant structures. With suboptimal processing parameters, features such as gaps can form in between tracks, which could cause porosity to form in parts produced with multiple layers. The experimental method used in this chapter made it difficult to quantify certain measurements, as tracks would overlap over each other, making it difficult to isolate individual melt pools and quantify their dimensions. To address this issue, a new technique was developed as described in the next section, allowing a better isolation of single tracks and their formation.

\subsection{Experiment B - Single-Lines on Recessed Plates Method}

\subsubsection{Results}

Topographical images of the single tracks from experiment B were compiled into a process map as a function of scan speed on the horizontal axis, and laser power on the vertical axis, as seen in Figure 27. Each image is comprised of three track formations, made using the same laser power and scan speed process parameters. The regions of the process map without an image signify that track formation was not successful at those corresponding parameters. Using the same axes as Figure 27, a process map was constructed using cross-sectional images obtained using the same parameters, as seen in Figure 28. On both figures, the optimal parameter combination used from the DOE method in chapter 5.1.1 was included as a figure in these process maps as a reference to the parameters used to obtain optimal bulk density for this specific batch of powder material.

An additional process map was created using measurements of the percentage of the length of continuously laid track observed against the total length of the track specified in the design of the experiment. Essentially, this process map documented the percentage of successfully fabricated track, and was called the line-build percentage. This process map can be seen in Table 13. In this table, the cells are identified in five different colours, used to identify the type of track formation that took place at the specified laser power and scan speed combination. 


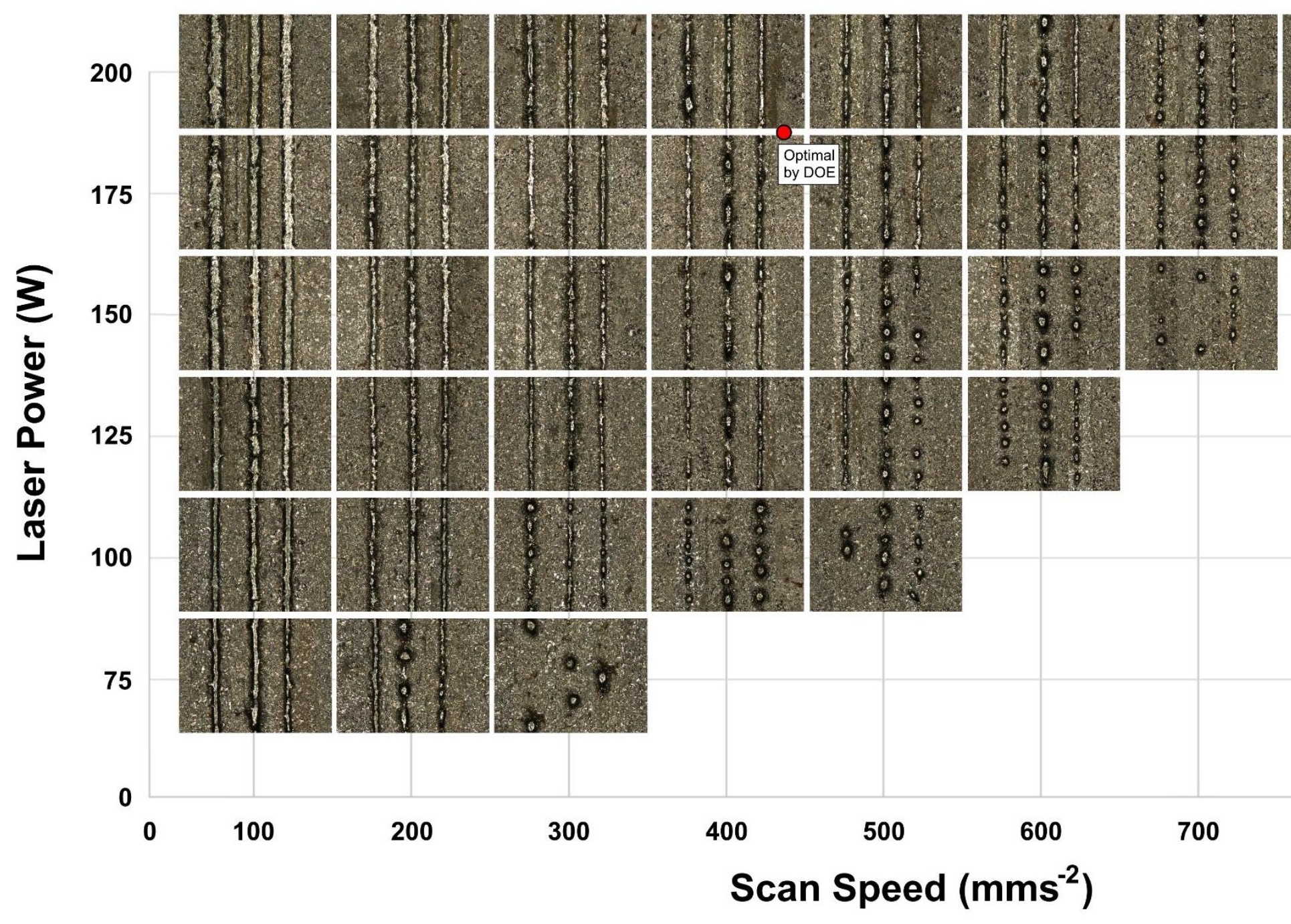

Figure 27. Experiment B (SS316L) - Topographical process map at 50um layer depth. The red dot show Renishaw recommended operating conditions. 


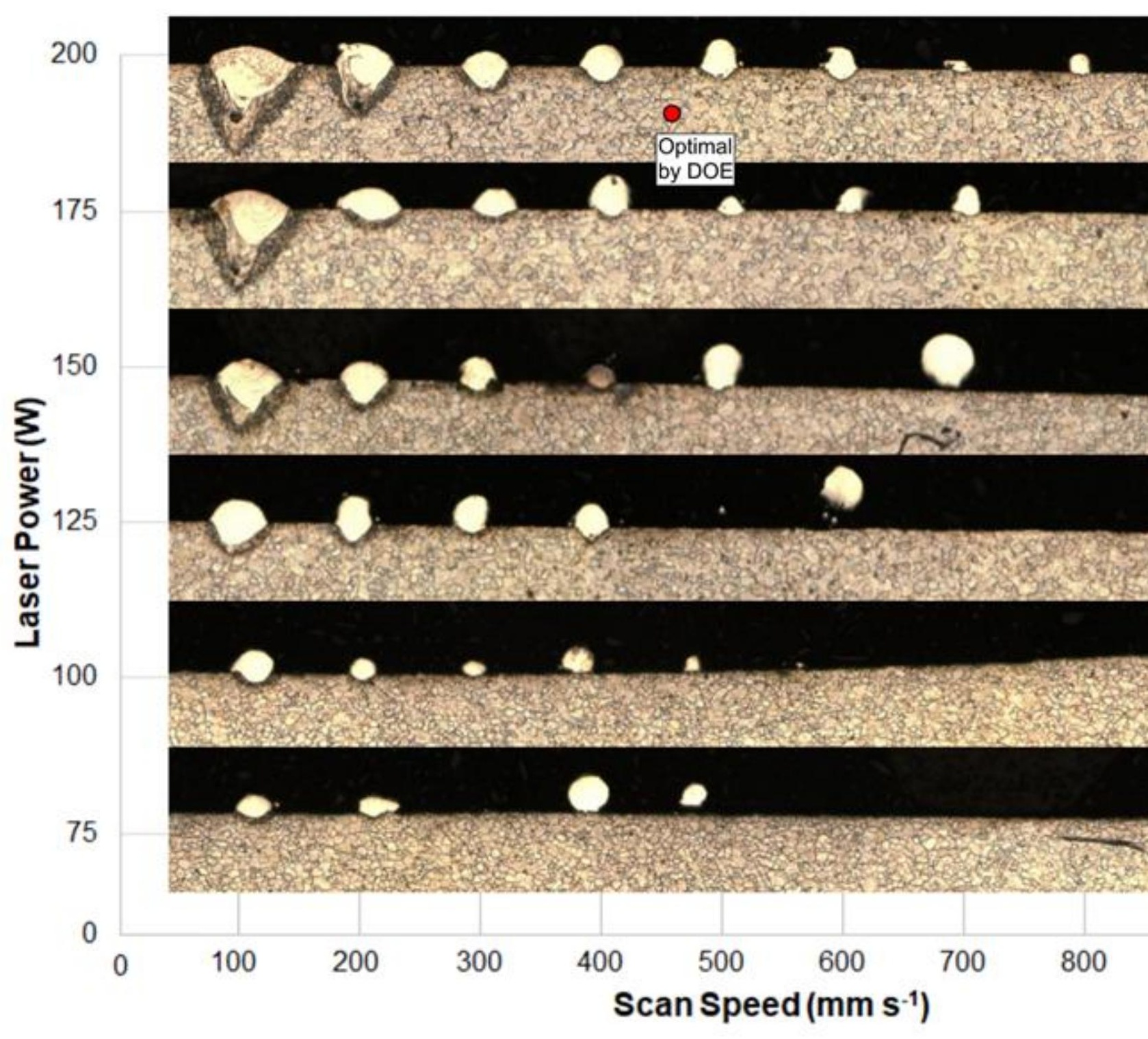

Figure 28. Experiment B (SS316L) - Cross-sectional process map at $50 \mu \mathrm{m} \mathrm{I}$ The red dot shows the shows the parameters used at the Renishaw recommended 0 


\subsubsection{Discussion}

Focusing on single track formations allowed for greater clarity in identifying the way in which tracks would form in relation to the energy input. At the high energy input ranges, where laser power was between $200 \mathrm{~W}$ to $150 \mathrm{~W}$, and very slow scan speeds, $100 \mathrm{mms}^{-1}$, tracks formed as continuous, smooth tracks with negligible variation in the pattern of the tracks formed, as observed in Figure 29i. These track-types have been annotated in Table 13 as the blue section.
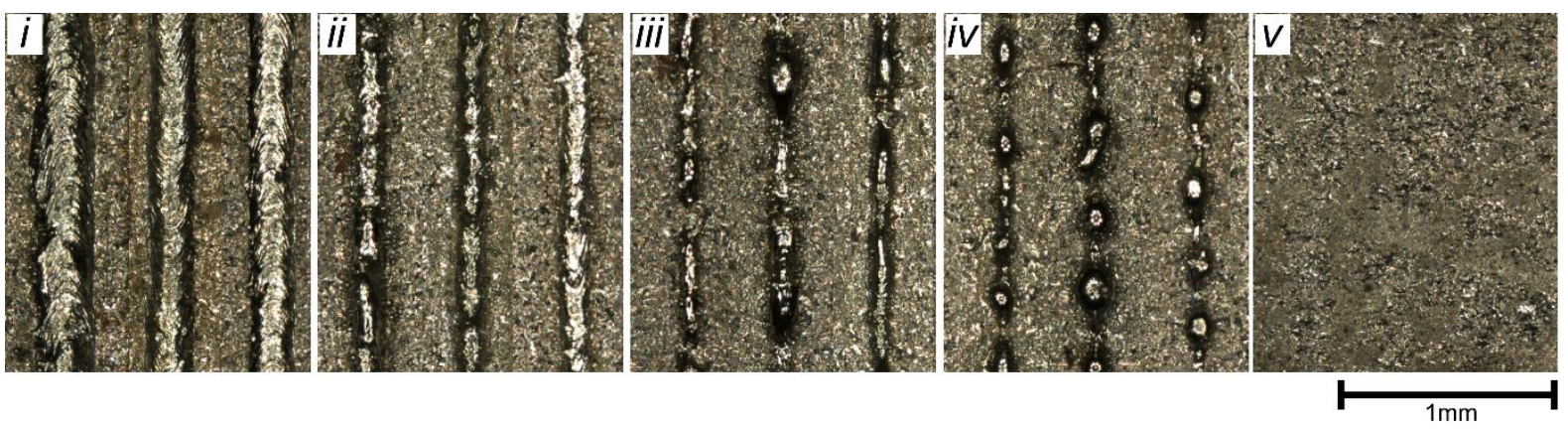

Figure 29. Experiment B (SS316L) -The five types of topographical tracks which formed during experiment. From left to right: i) continuous/regular tracks (200W, $\left.100 \mathrm{mms}^{-1}\right)$, ii) continuous/irregular tracks $\left(150 \mathrm{~W}, 400 \mathrm{mms}^{-1}\right)$, iii) discontinuous/irregular tracks (150W, 400 $\left.\mathrm{mms}^{-1}\right)$, iv) balling $\left.\left(175 \mathrm{~W}, 700 \mathrm{mms}^{-1}\right), \mathrm{v}\right)$ build failure $\left(100 \mathrm{~W}, 1000 \mathrm{mms}^{-1}\right)$.

\begin{tabular}{|c|c|c|c|c|c|c|c|c|c|c|c|}
\hline \multirow{6}{*}{ 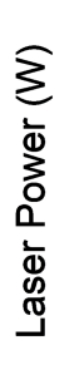 } & 200 & 100 & 100 & 100 & 97.4 & 97.7 & 82.2 & 73.1 & 58.5 & 55.5 & 54.7 \\
\hline & 175 & 100 & 100 & 98.6 & 91.9 & 91.5 & 77.3 & 57.9 & 45.6 & 39 & 29.8 \\
\hline & 150 & 100 & 100 & 98.5 & 89.7 & 82.1 & 53.3 & 12.8 & 0 & 0 & 0 \\
\hline & 125 & 100 & 98.3 & 95.9 & 79.7 & 58.2 & 45.2 & 0 & 0 & 0 & 0 \\
\hline & 100 & 100 & 97.1 & 73.9 & 35.7 & 16.4 & 0 & 0 & 0 & 0 & 0 \\
\hline & 75 & 100 & 68.9 & 13.7 & 0 & 0 & 0 & 0 & 0 & 0 & 0 \\
\hline & & 100 & 200 & 300 & 400 & 500 & 600 & 700 & 800 & 900 & 1000 \\
\hline & & & & & & $\mathrm{SP}$ & $\mathrm{mr}$ & & & & \\
\hline
\end{tabular}

Table 13. Experiment B (SS316L) - Process Map with line build percentages, divided into sections, i) blue - continuous/regular tracks, ii) green continuous/irregular tracks, iii) yellow - discontinuous/irregular tracks, iv) orange balling, v) red - build failure.

\section{Continuous Regular Tracks and Keyhole Mode Melting}

The tracks would build with a consistent width, and had a rounded-front pattern similar to the tracks formed at the high power and high exposure time samples in Experiment A, namely 
samples 4 (Figure 23) and 6 (Figure 25). This seems to suggest that the high energy input suitably melts the powder layer underneath the laser area and the melts wets to the substrate. Additionally, the melt also spreads out and flattens, creating the smooth surfaces observed in Figure 29i. The line-build percentage for each repetition of these build conditions was $100 \%$, indicating that there was complete melting of the material that came in contact with the lasers' path.

These would be regarded as ideal formations for laser-additive manufacturing from the topographical results. However, the cross-sectional results reveal that these tracks have Vshaped penetrations into the substrate, with pores that form near the bottom of such penetrations. Examples of both phenomena can be seen in the same cross-section on the right-hand side of Figure 30. It is assumed that vaporisation of the melt would occur due to the excessively high temperatures, and that keyhole mode melting had occurred in these tracks, as discussed in chapter 3.3.4 Parts made using these parameters could exhibit porosity, although remelting of previous layers may eliminate the effect of porosity caused by keyholing. Spreading of the melt due to good wetting conditions is further evidenced here, as these tracks were found to have the widest cross-sections.
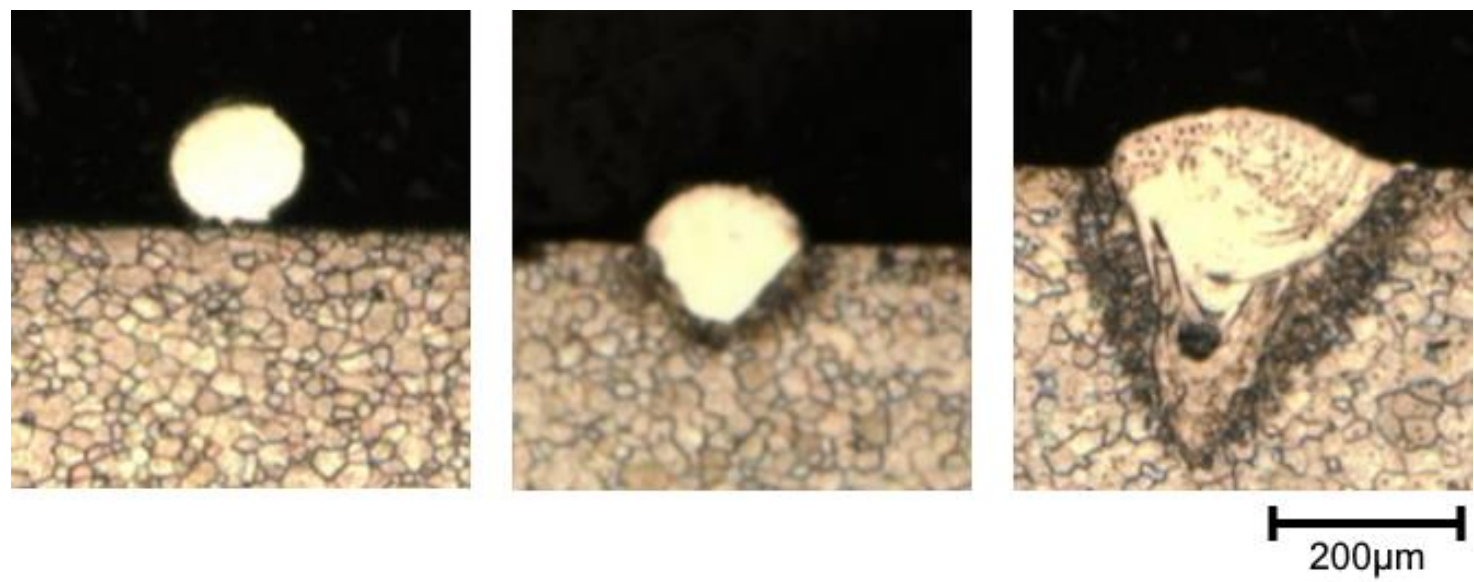

Figure 30. Experiment B (SS316L) - The three types of cross-sectional tracks formed during experiment. Left) $125 \mathrm{~W}, 600 \mathrm{mms}^{-1}$, Middle) 200W, $300 \mathrm{mms}^{-1}$, Right) $150 \mathrm{~W}, 500 \mathrm{mms}^{-1}$

\section{Continuous Irregular Tracks}

Tracks would remain continuous under conditions of high laser power, between $200 \mathrm{~W}$ to $75 \mathrm{~W}$, and relatively low scan speeds, between $500 \mathrm{mms}^{-1}$ and $100 \mathrm{mms}^{-1}$, with the line build 
percentages ranging between $100 \%$ and $97 \%$. However, track formation in this range of the process map would not exhibit the homogenous, smooth patterns seen in the previous track formations, as observed in Figure 29ii. Kinks and slight distortions in the track were observed, unlike the smooth and consistent tracks seen in Figure 29i.

The occurrence of these kinds of distortions were generally observed to increase with scan speed, as seen in Figure 31. These distortions could be attributed to the melt not having enough time to wet to the substrate due to fast scan speeds, or the melt not achieving a sufficient temperature to wet to the substrate, or a combination of both factors.

The cross-sectional results show that tracks made at these conditions have a large degree of penetration into the substrate, often with half or more of the melt bead underneath the substrate. The wetting angle is small, though not as minute as those observed for continuous, regular tracks, which were almost completely flat. This suggests that the melt achieved a considerable degree of wetting and spreading over the substrate. Since the tracks retained a $100 \%$ to near- $100 \%$ line-build percentage, these tracks are referred to as continuous and irregular tracks.

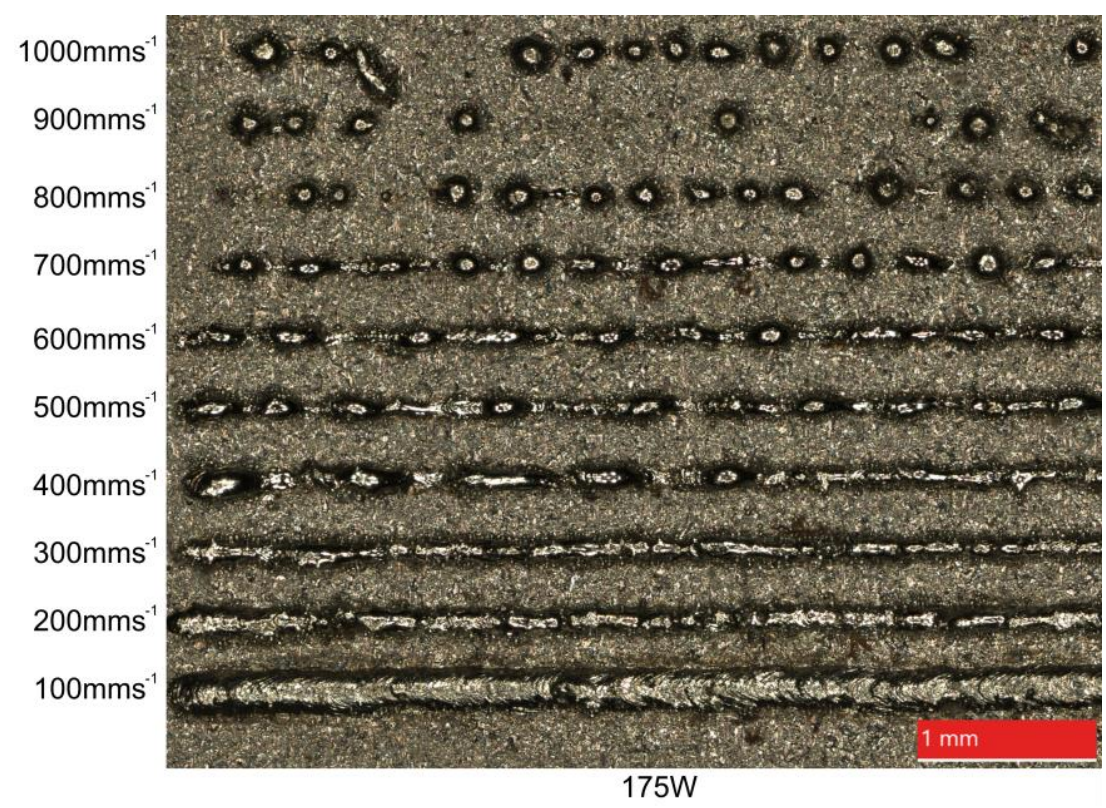

Figure 31. Experiment B (SS316L) - Variation of the melt pool stability with scan speed. 
These conditions are considered the ideal types of track formations conducive to creating ideal, near-fully dense parts. The results from the DOE method correlate with this statement, as is evidenced in Figure 28, where tracks made with similar processing parameters to the

DOE method, indicated by the red dot, were found to produce ellipsoidal, bead-shaped cross-sections. They have been annotated in Table 13 as the green section.

\section{Balling}

At higher scan speeds, tracks would begin to fragment into a mixture of cylindrical bead structures and large spherical droplets or bulges, as observed in Figure 29iii. The line-build percentage would range between $95.9 \%$ and $73.1 \%$, leaving the track full of gaps. The wettability of the melt pool would decrease, resulting in the formation of bulges along the track, as can be seen in the $400 \mathrm{mms}^{-1}$ track in Figure 31 .

The wettability of the melt would decrease with less power or increased scan speed. This formation is referred as being discontinuous and irregular. The range of parameters would form as a narrow band in the process map, and have been annotated in Table 13 as the yellow section.

At even higher scan speeds, the tracks would only form as a series of solidified droplets, with very low line-build percentages, ranging between $68.9 \%$ and $13.7 \%$. These track formations can be seen in Figure 29iv, and have been annotated in Table 13 in the orange section.

The balling phenomenon was repeatedly observed both in the topographical and crosssectional results. With this parameter combinations, powder was still able to melt, although the volume of the melt would be comparatively low when compared to tracks with higher input energy. The lower volume of liquid that forms has reduced contact area with the solid substrate, which leads to poor wetting, flow and spreading conditions. Additionally, since the energy input is decreased, the temperature of the melt is also comparatively low. Surface tension of most liquids generally increases with decreasing temperature. This is due to cohesive forces of liquid metals increasing with reduced molecular thermal activity, leading 
to worsened wettability. [106]. The viscosity of the melt becomes considerably high, severely limiting liquid flow, and this in turn decreases the rheological performance of the melt with solid surfaces [107]. Surface tension at such conditions becomes the dominant force controlling the shape of the melt, and the tracks solidify as a series of spheroidal, broken-up structures. This is further evidenced in the cross-sectional results, where a spherical melt bead barely penetrates into the previous substrate layer, resulting in a large contact angle. At the highest scan speeds and lowest laser power range, tracks would either fail to build at all or very sparingly. This range of processing parameters has been annotated in Table 13 in the red section. At these parameters, only a small volume of molten material would form, with limited contact to the substrate.

\section{Verification of Results}

As part of the verification for the method used in this experiment, the results from the width and depth measurements of the tracks formed were compared to the results from a similar experiment carried out by Bertoli et al, [31]. In the Bertoli et al. experiment, single track formations were fabricated using a custom SLM system with a 1070nm Yb-fiber laser. These tracks were made using stainless steel $316 \mathrm{~L}$ powder, and the laser power and scan speed used to create these tracks were varied to investigate their effect on the formation of the melt pool. The single tracks were constructed Onto a stainless steel 316L substrate.

The average track width and track depth were plot against laser power and scan speed. The track width plots can be seen in Figure 32 and Figure 33, whilst the plots for track depth can be seen in Figure 34 and Figure 35.

A linear or exponential line of best fit was drawn using these results to investigate the relationship between scan speed and the width or depth of the track. The results from the Bertoli el al. experiment were taken from tracks constructed using laser power settings of $100 \mathrm{~W}$ and $200 \mathrm{~W}$, with scan speed ranging between $600 \mathrm{mms}^{-1}$ and $100 \mathrm{mms}^{-1}$. These results were marked on each plot using a dark grey circle and also had a line of best fit drawn, distinguished from the other trendlines as a dashed, grey line. 
It was generally observed that track width and depth would increase as scan speed decreased at all laser power settings. This observation was also true for the Bertoli et al. results. Track width and depth would also increase with laser power, obtaining large width and depth values for the same scan speed at higher power settings.

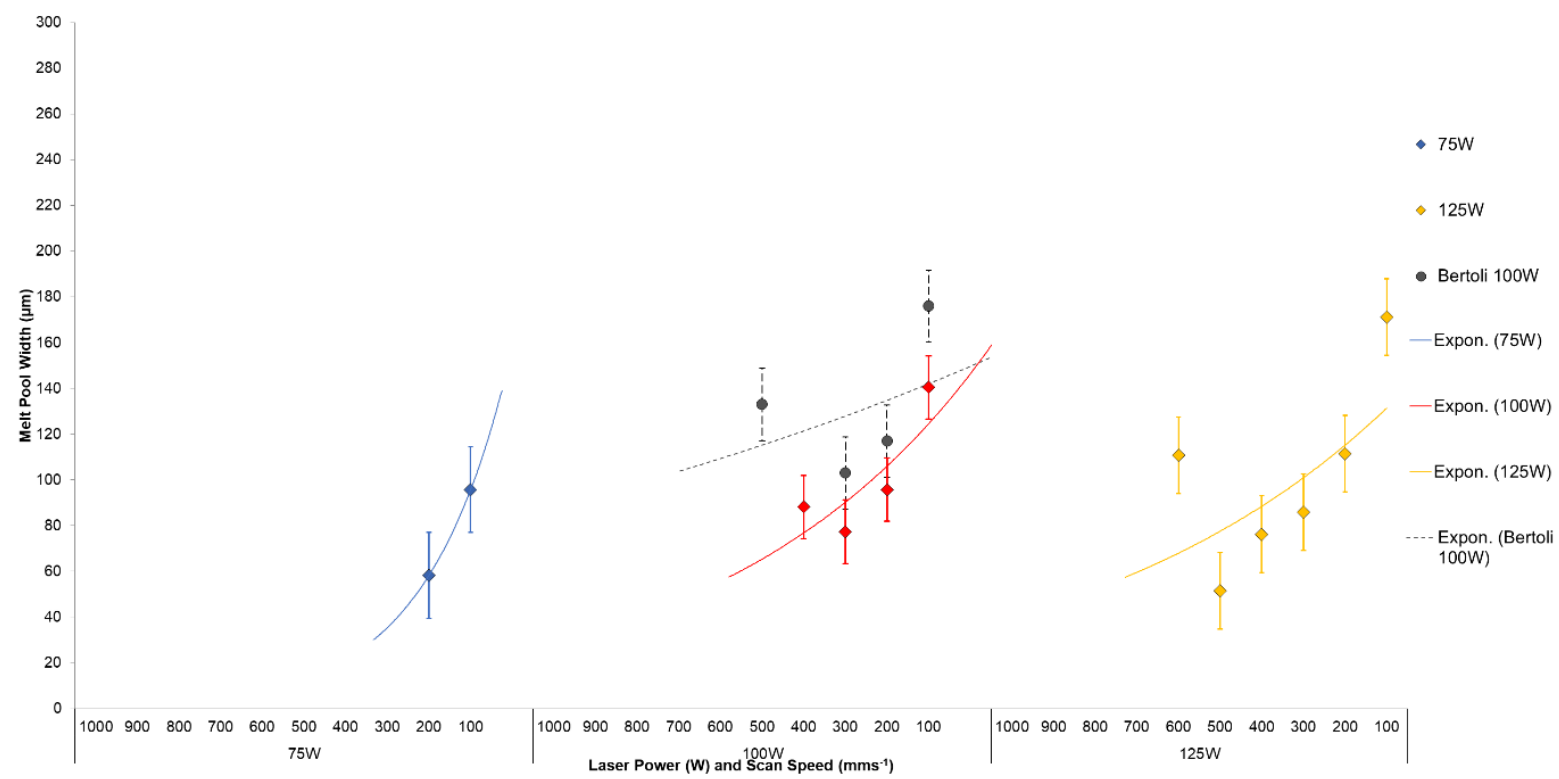

Figure 32. Experiment B (SS316L) - Track width at the 75-125W range compared to results from Bertoli et al, [31].

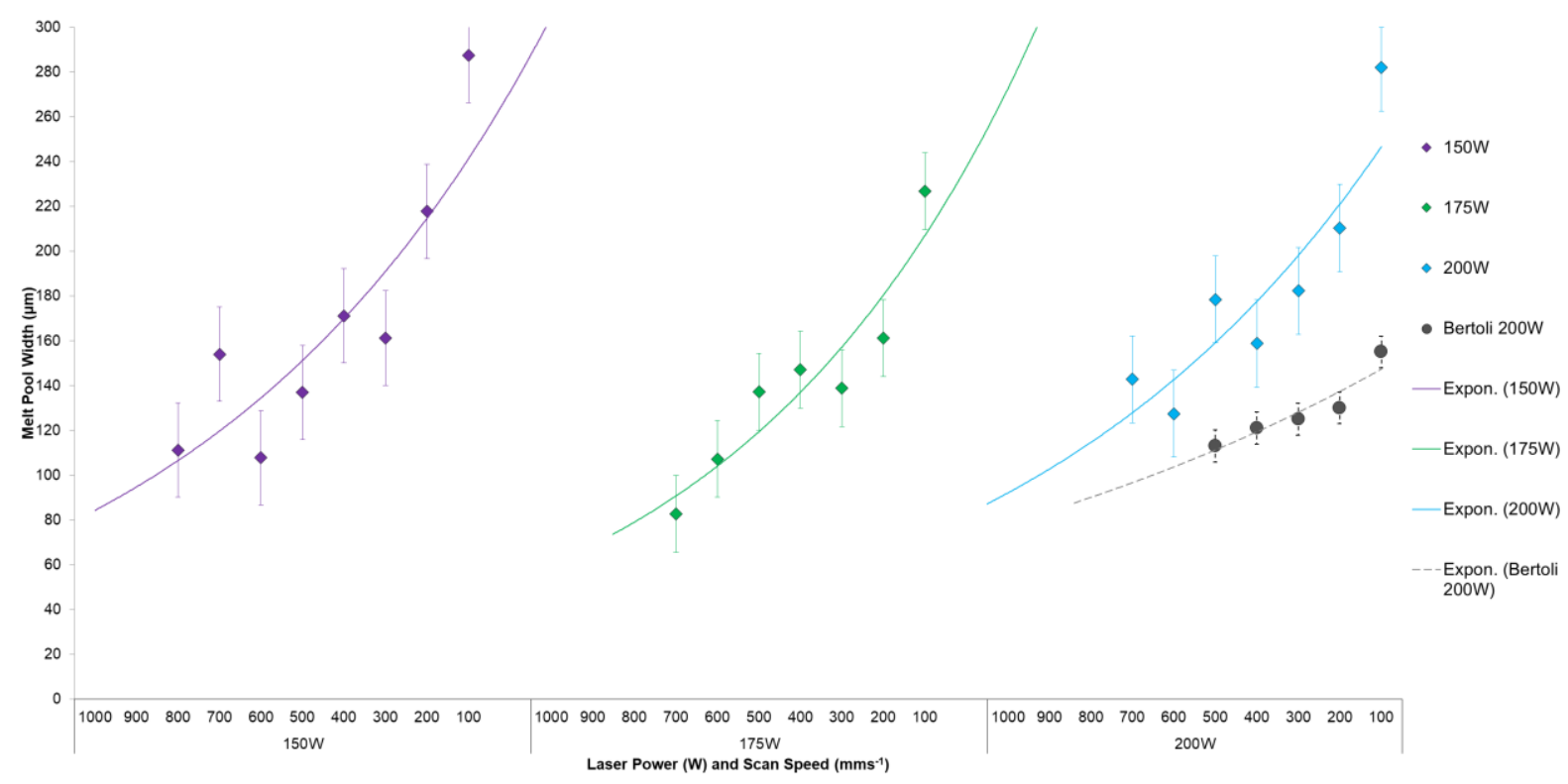

Figure 33. Experiment B (SS316L) - Track width at the 150-200W range compared to results from Bertoli et al, [31]. 


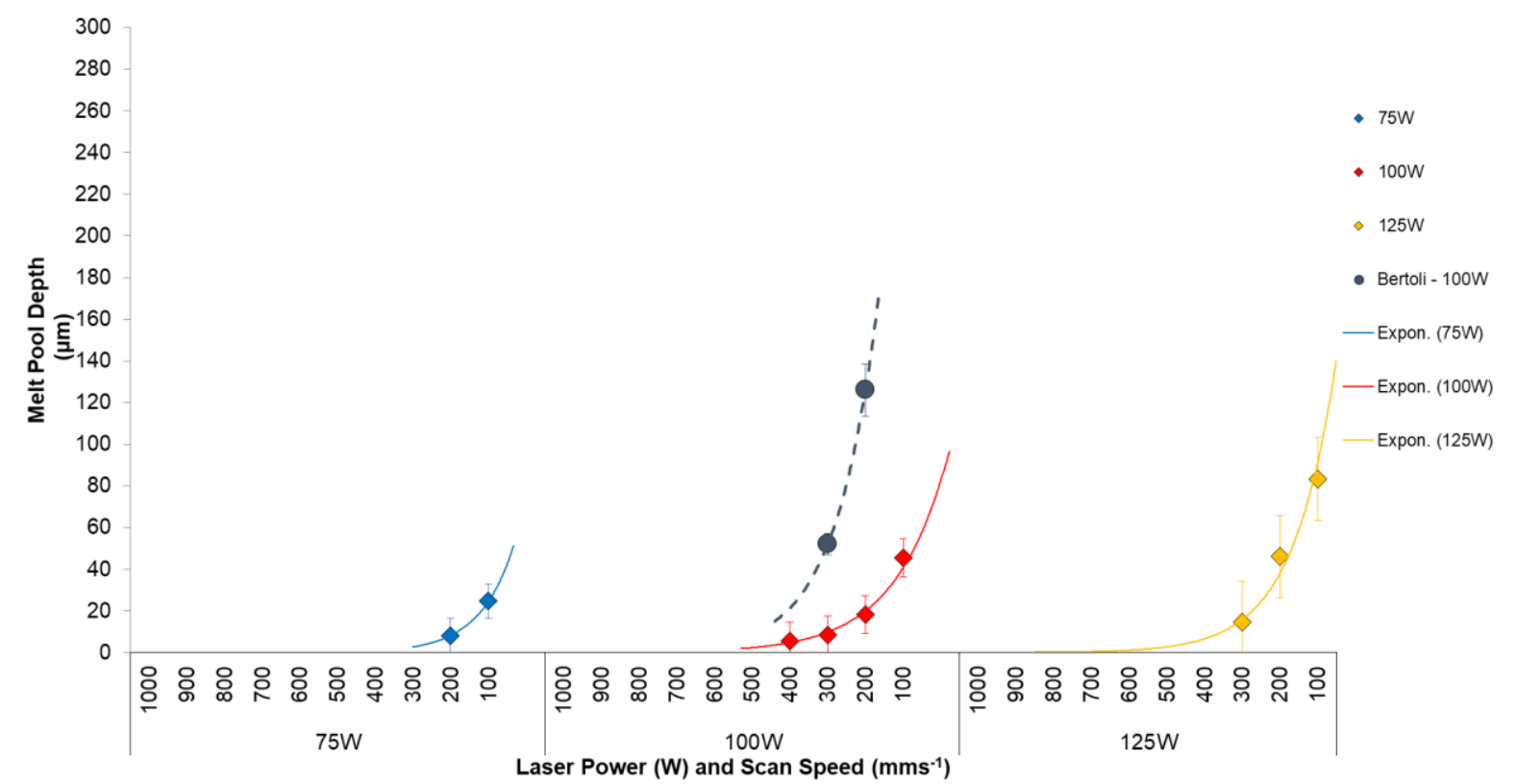

Figure 34. Experiment B (SS316L) - Track depth at the 75-125W range compared to results from Bertoli et al, [31].

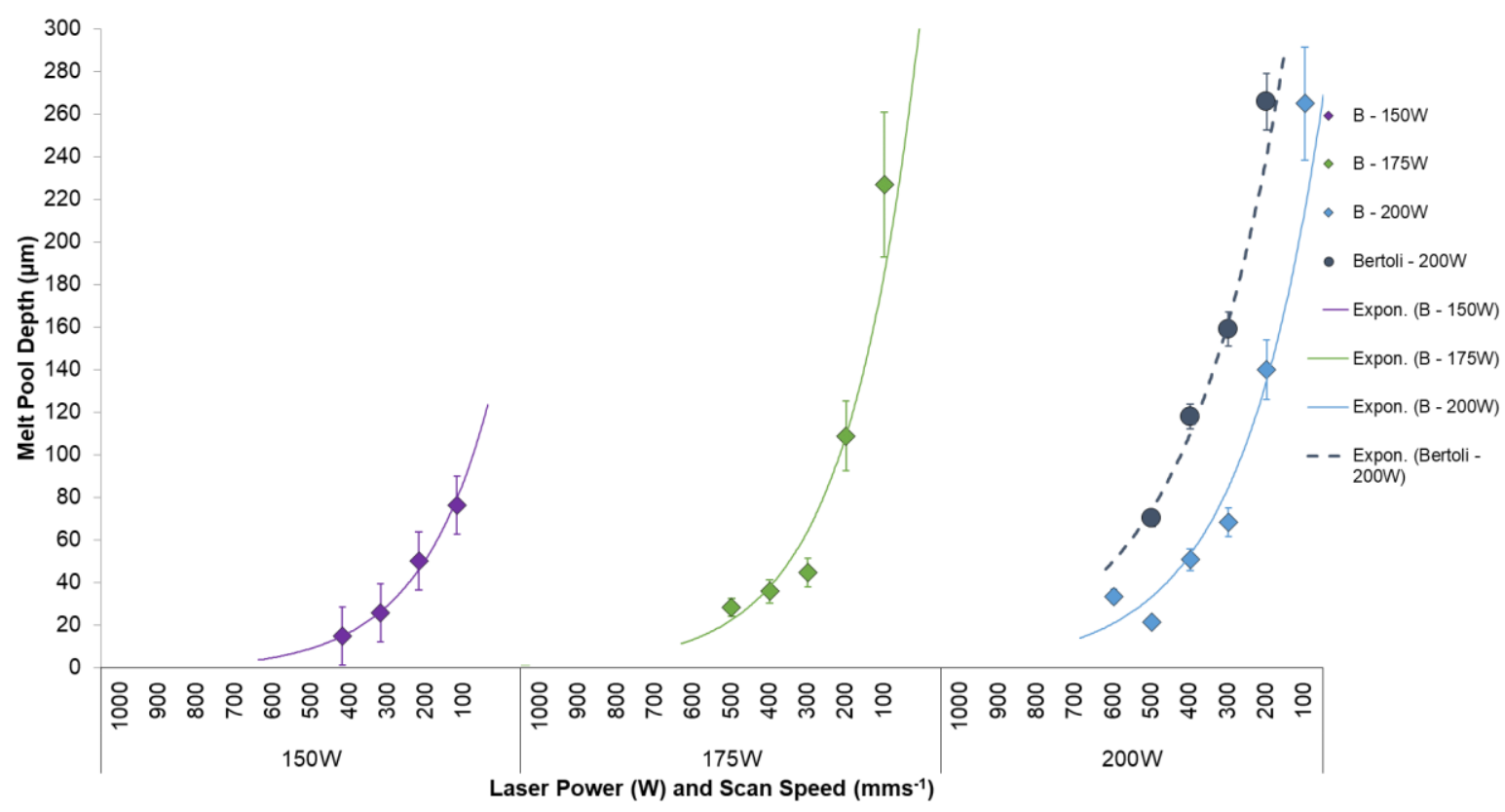

Figure 35. Experiment $B$ (SS316L) - Track depth at the 150-200W range compared to results from Bertoli et al, [31].

At $100 \mathrm{~W}$ and $200 \mathrm{~W}$, the width values from the Bertoli et al. experiment were comparable to the results obtained in experiment $B$, as seen in the middle section of Figure 32 and the right most section in Figure 33. At 100W, the depth measurements obtained from Bertoli et al. 
were considerably larger than those obtained for Experiment B, as seen in the middle section of Figure 34 . At $200 \mathrm{~W}$, the depth results were slightly larger but were more comparable to the depths observed in Experiment B, as seen in the right most section of Figure 35.

The depth values achieved in this experiment were also compared to those generated by a deep penetration melting empirical model, developed by Gladush and Smurov, [108], used for predicting keyhole formation during laser welding. This model was later referenced and slightly modified by King et al, [26], to be used to predict keyhole formation during SLM. The equation included the material property of absorptivity as a modification which more accurately represents the amount of powder absorbed by the powder material. The model is based on a relatively simplistic analytical solution (the Rosenthal welding solution) can give a relationship between depth of penetration to the laser power and scanning speed:

$$
d=\frac{A P}{2 \pi k T_{b}} \ln \left(\frac{\sigma+\frac{D}{v}}{\sigma}\right)
$$

Where $d$ was depth (m), $A$ was absorptivity (dimensionless), $P$ was laser power $(\mathrm{W}), k$ was thermal conductivity $\left(\mathrm{Wm}^{-1} \mathrm{~K}^{-1}\right), T_{B}$ was the boiling point of the material $(\mathrm{K}), \sigma$ was the spot size of the laser beam and $v$ was the scanning speed of the laser $\left(\mathrm{ms}^{-1}\right)$.

The material properties of stainless steel 316L were taken from Gladush and Smurov, [108], and are listed below in Table 14.

\begin{tabular}{|c|c|c|c|}
\hline Symbol & Property & Value & Units \\
\hline$\rho$ & Density & 7980 & $\mathrm{kgm}^{-3}$ \\
\hline$h_{s}$ & Enthalpy at melting & 1200000 & $\mathrm{Jkg}^{-1}$ \\
\hline$A$ & Absorptivity & 0.4 & \\
\hline$D$ & Diffusivity & 0.000006 & $\mathrm{~m}^{2} \mathrm{~s}^{-1}$ \\
\hline$T_{m}$ & Temperature at melt & 1650 & ${ }^{\circ} \mathrm{K}$ \\
\hline$C$ & Specific heat capacity & 700 & $\mathrm{Jkg}^{-1} \mathrm{~K}^{-1}$ \\
\hline$K$ & Thermal conductivity & 31 & $\mathrm{WmK}$ \\
\hline$P$ & Power & $100-400$ & $\mathrm{~W}$ \\
\hline$U$ & Speed & $50-4000$ & $\mathrm{~ms}^{-1}$ \\
\hline$\sigma$ & Laser spot size & 0.000035 & $\mathrm{M}$ \\
\hline$T_{b}$ & Temperature at boil & 3500 & ${ }^{\circ} \mathrm{K}$ \\
\hline
\end{tabular}

Table 14. Values used to calculate values for penetration depth equation from Gladush and Smurov , [108]. 
The values of the measured experimental and predicted melt pool depths were compared in Figure 36 and Figure 37 below. A fairly good correlation can be seen between the predicted and measured depths, except at the higher laser powers of 200W and 150W, as seen in the two right-most plots in Figure 37. At these power settings, the data obtained from the experiments follows the equation data quite well for the scan speeds between $600 \mathrm{mms}^{-1}$ and $300 \mathrm{mms}^{-1}$. At the slower speeds of $200 \mathrm{mms}^{-1}$ and $100 \mathrm{mms}^{-1}$, the measured track depth increased drastically for both these power settings. This was due to the very deep keyhole formations were observed in most of the samples, due to the vaporisation effect discussed in the previous section.

It should be noted that since a mild carbon steel substrate was used, any degree of penetration into the substrate would cause the stainless steel $316 \mathrm{~L}$ powder melt to combine with the mild steel. As keyholing would cause the melt to penetrate further into the substrate, the material properties of the melt would change drastically, and would no longer resemble the input variables used in the empirical equation. However, the trends displayed by the measured and the analytical solution are in relatively good agreement. 


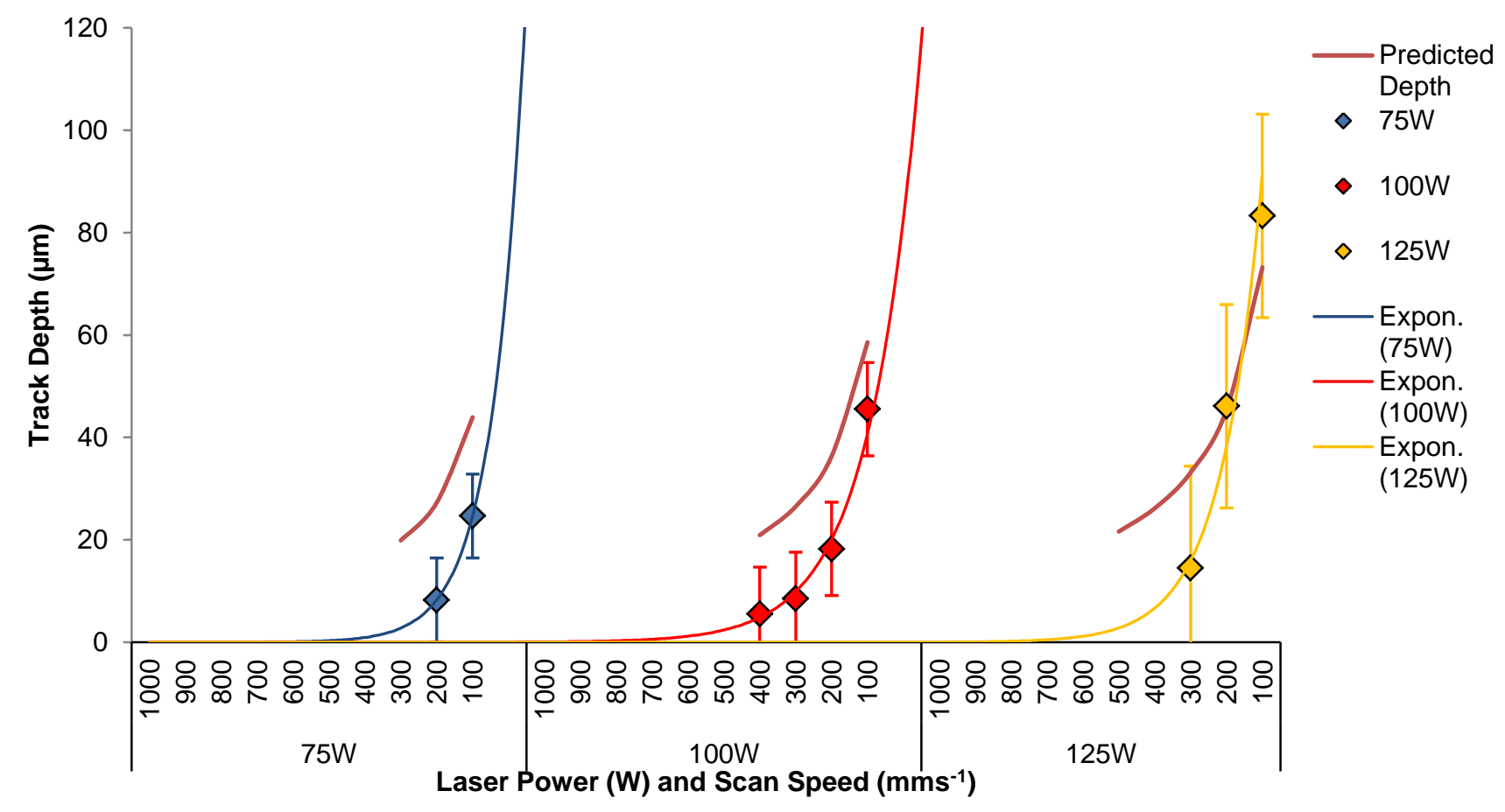

Figure 36. Experiment B (SS316L) - Comparison of measured and predicted melt pool depths at the 75-125W range according to equation from Gladush and Smurov, [108].

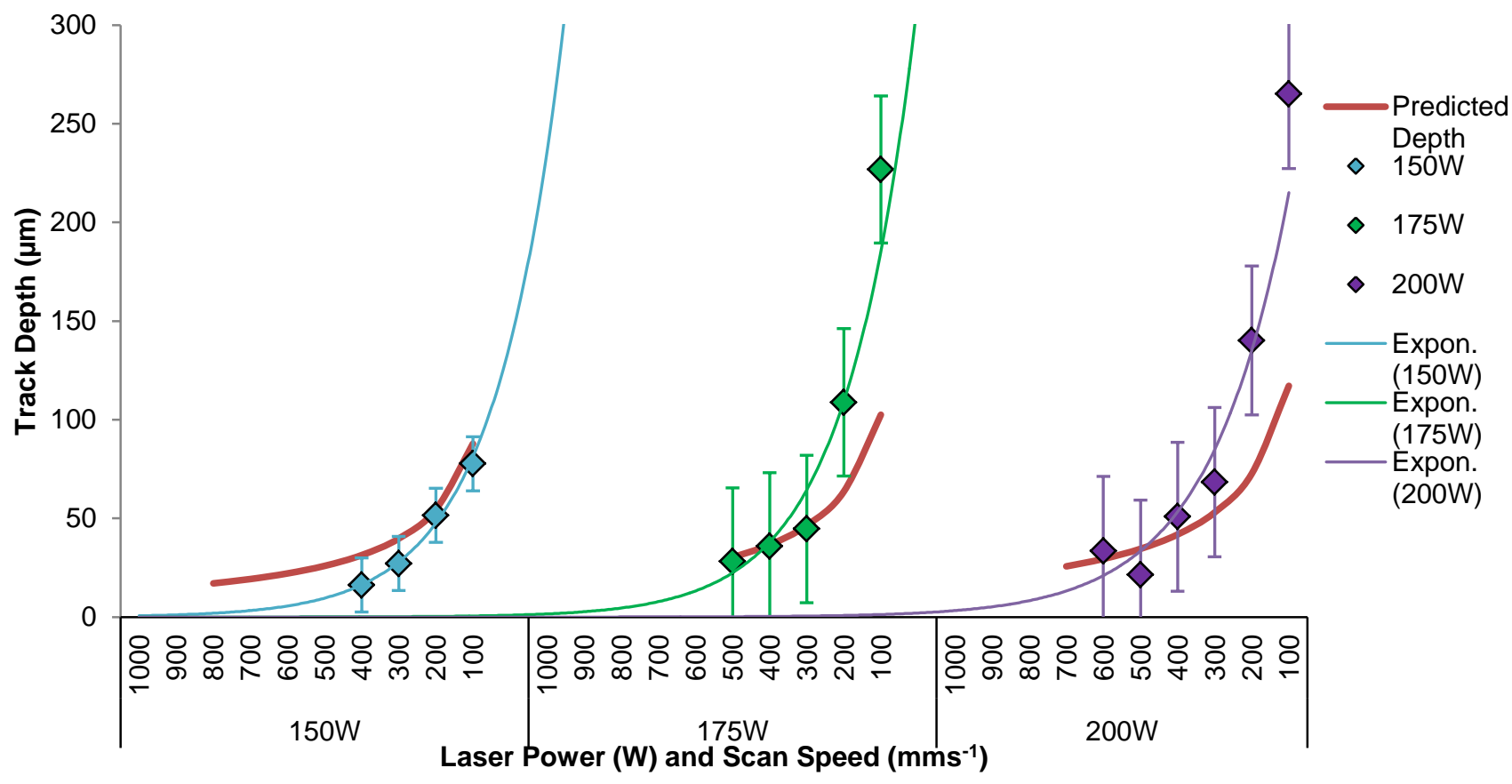

Figure 37. Experiment B (SS316L) - Comparison of measured and predicted track depths at the 150-200W range according to equation from Gladush and Smurov, [108]. 
The line build percentage values and depth-to-width ratios were used to build two contour maps, as seen in Figure 39 and Figure 40, respectively. The depth-to-width ratio, also known as the aspect ratio, is commonly used to distinguish between conduction mode and keyhole mode melting during laser interaction with metals, [109], where keyhole mode melting is expected to be observed at high values. The exact value for the depth-to-width ratio transition point can vary according to the material and equipment used. A generic assumption used for welding and melting of metals is that any track formed with a depth-toweld ratio less than 0.5 has undergone conduction mode melting, [110]. A marker was used in both these contour maps to indicate the laser power and scan speed used to create the optimal density from the DOE performed at the beginning of the chapter.

In Figure 39, optimal line build percentages are seen at the left-most portion of the contour map, where the line-build percentage for the tracks was $100 \%$. At high laser power settings, ranging between $150 \mathrm{~W}$ and $200 \mathrm{~W}$, scan speed can range between $100 \mathrm{mms}^{-1}$ and $300 \mathrm{mms}^{-1}$ to obtain $100 \%$ on line build percentage values. This range is limited to the $100 \mathrm{mms}^{-1} \mathrm{scan}$ speed at lower power settings of $125 \mathrm{~W}$ and $100 \mathrm{~W}$. The parameters used for the optimal DOE fell between a $95 \%$ and $90 \%$ in the same contour plot. Tracks built in this region were continuous but distortions, gaps and bulges were observed to form throughout the track, which could be attributed to incomplete wetting of the track, as seen below in Figure 38. In Figure 40, the depth to width ratios at the left most corner of the contour map are exceptionally high, in the regions where keyhole formation was commonly observed. The parameters used for the optimal DOE fell into a region where the depth-to-width ratio indicated that conduction mode melting would take place. 

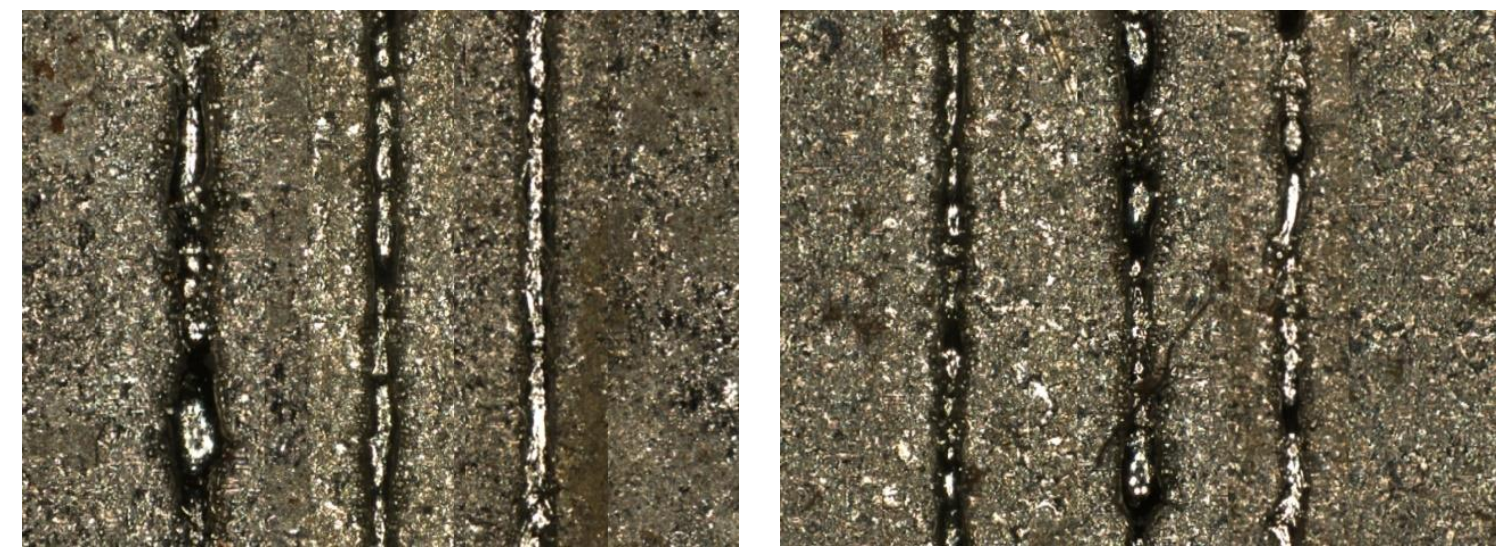

Figure 38. Experiment B (SS316L) - Topographical images of tracks built using parameters similar to the DOE optimal parameters $\left(180 \mathrm{~W}, 433 \mathrm{mms}^{-1}\right)$.

left) $200 \mathrm{~W}, 400 \mathrm{mms}^{-1}$, right) $175 \mathrm{~W}, 500 \mathrm{mms}^{-1}$.

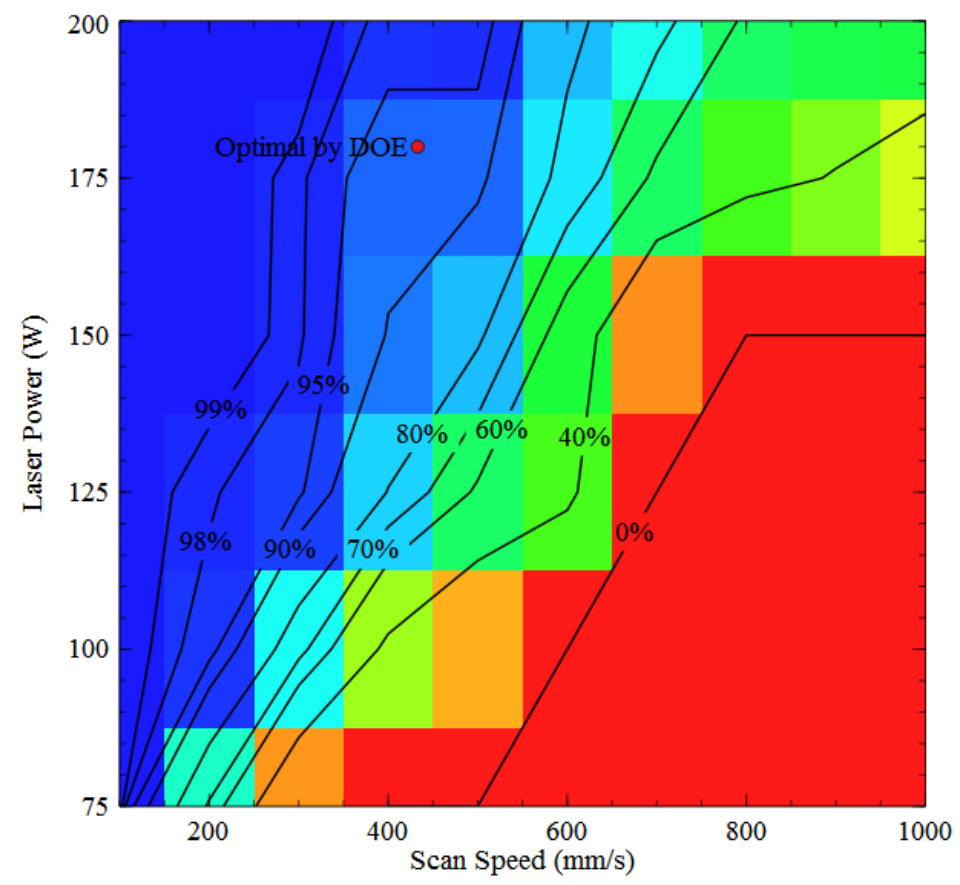

Figure 39. Experiment B (SS316L) - Contours of build ratios of single tracks. 


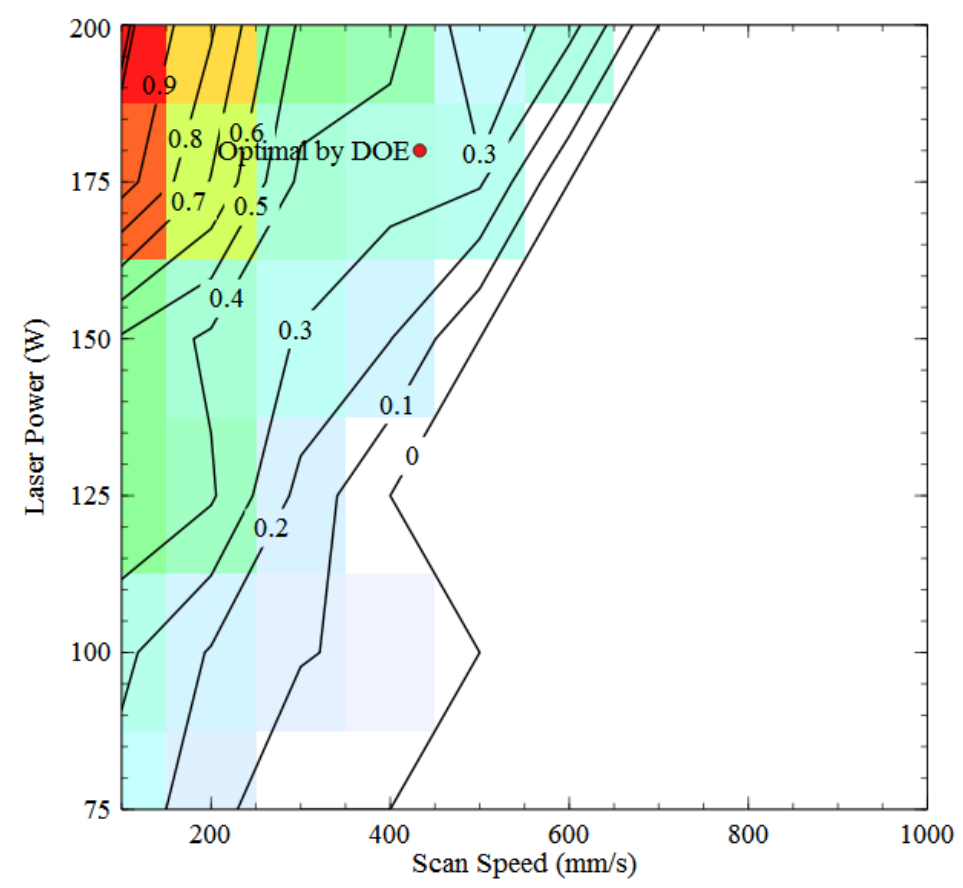

Figure 40. Experiment B (SS316L) - Contours of depth to width ratio of single tracks.

\subsubsection{Conclusions}

As similarly observed in Experiment $A$, a systematic variation in track morphology was observed when the laser power and scan speed process parameters were altered. Tracks built using exceptionally high scanning speeds or low laser power resulted in fragmentation and balling due to insufficient melting or wetting to the previous layer. High laser power and low scanning speed resulted in vaporisation of the material, causing keyhole formation. This can cause porosity to form below the surface of the track. Although keyholing was observed at these regions, it should be noted that the depth-to-width ratios at these regions were slightly above 1 , indicating that the level of keyholing is relatively low.

The results obtained from Experiment B compared well to those from Bertoli et al, [31], as well as following the trends given by empirical/analytical solutions. Similarly, they also compliment the DOE optimal parameters, in that keyholing was avoided at the laser power and scan speed range where the DOE parameters lie. Track fragmentation and balling were also avoided in this range, factors which are detrimental to part density.

From the results in this experiment, within the range of the processing parameters used, the optimal combination of laser power and scan speed would have to be $200 \mathrm{~W}$ and $300 \mathrm{mms}^{-1}$ 
respectively. Although the track produced at these settings had irregularities, keyhole mode melting was avoided using these settings, which would reduce the chance for porosities to form. At faster speeds, track instability would increase, whilst at lower power, line build percentage would decrease. 


\subsection{Experiment C - Verification of Single-Track Crucible Methodology}

\subsubsection{Results}

The topographical images taken of the melt tracks for Experiment $\mathrm{C}$ were compiled into a four process maps, one for each layer thickness used $(50 \mu \mathrm{m}, 100 \mu \mathrm{m}, 150 \mu \mathrm{m}$ and $200 \mu \mathrm{m})$, and can be seen in Figure 41, Figure 43, Figure 45 and Figure 47. Since the scan speeds used for each sample varied so greatly, the process maps were plotted as a function of laser power in the vertical axis, and surface energy density in the horizontal axis. It should be noted that the scan speed used for the tracks increases from left to right in the process maps. Each image comprises of four lines which are a repetition of the same power and scan speed. A red dot was included in Figure 41, referencing the optimal processing parameters used for obtaining bulk density in chapter 5.1.2

Cross-sections of the melt tracks are shown in Figure 42, Figure 44, Figure 46 and Figure 48 , and are presented in an identical manner to the topographical results. The line build percentages for each set of parameters can be found in Table 12. 


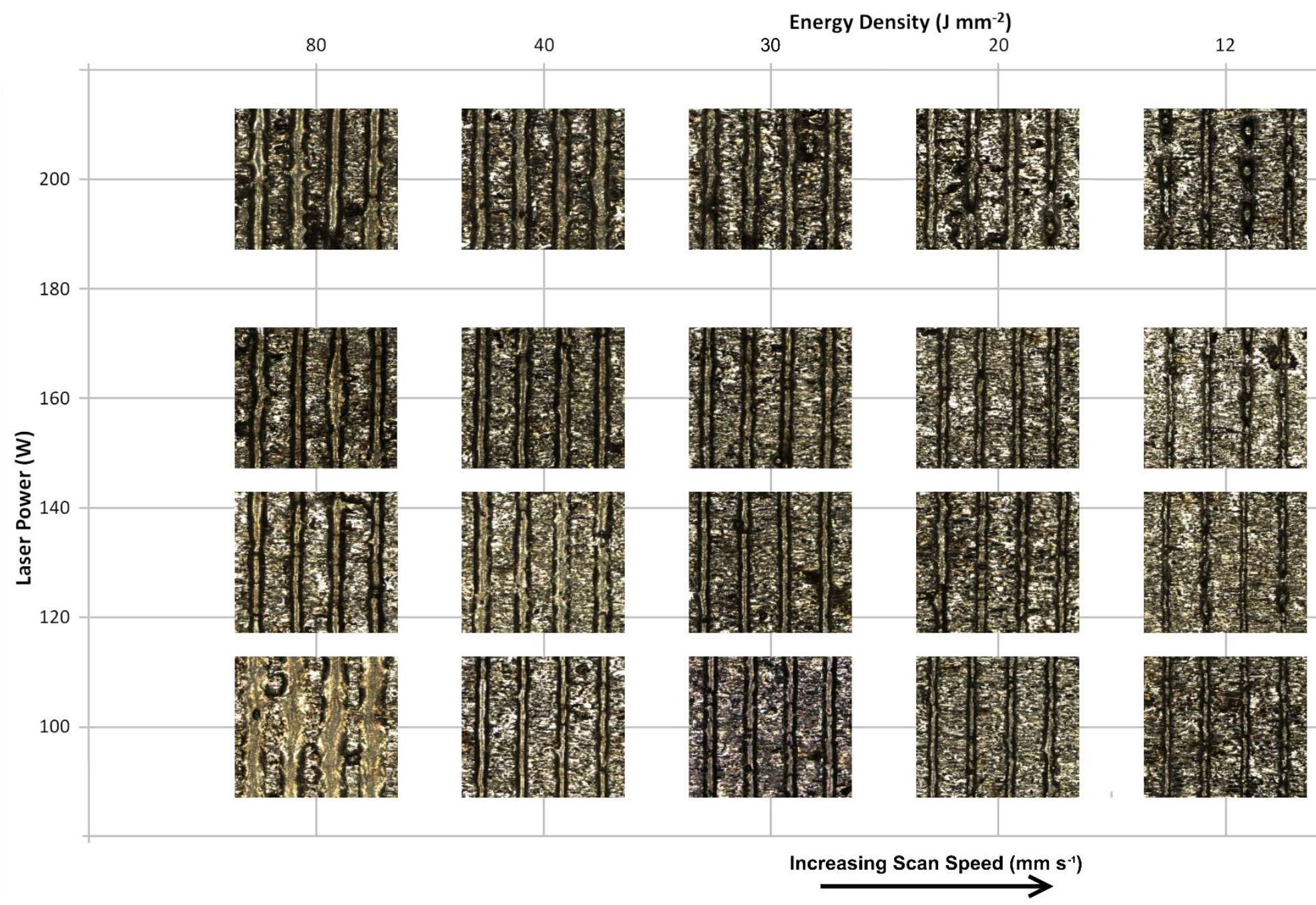

Figure 41. Experiment C (SS316L) - Topographical process map at $50 \mu \mathrm{m}$ 


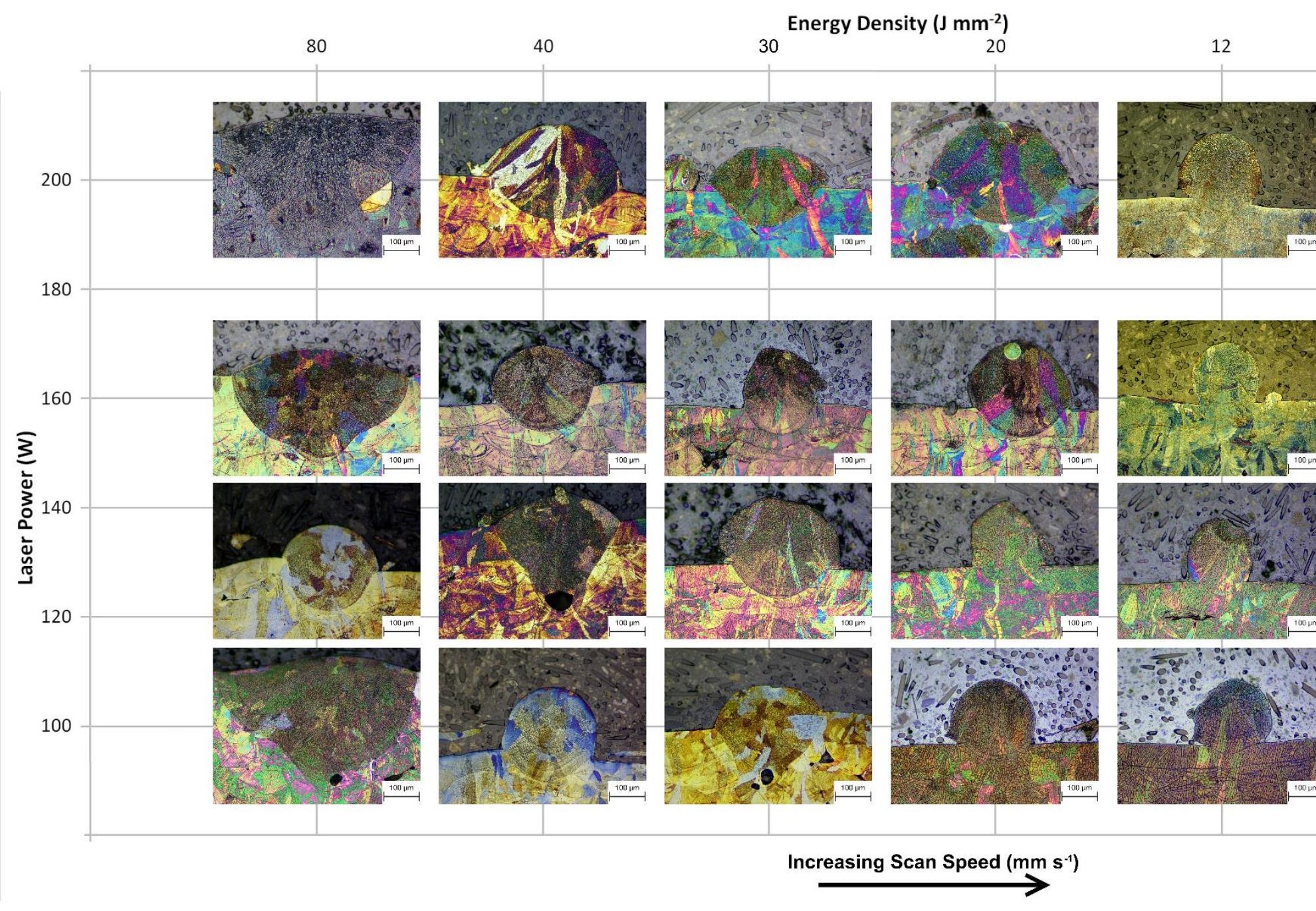

Figure 42. Experiment C (SS316L) - Cross-sectional process map at 50 $\mu \mathrm{m}$ 


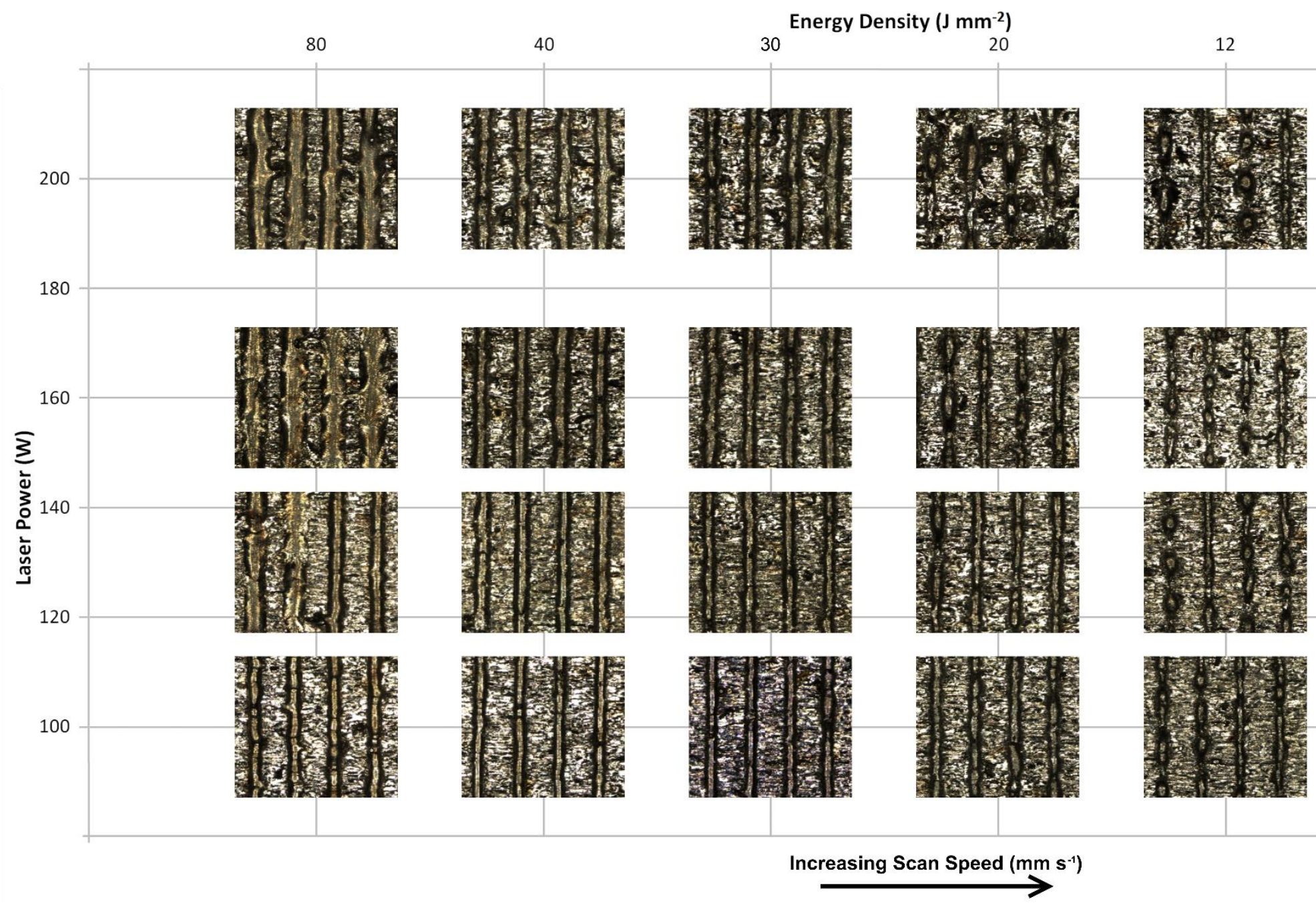

Figure 43. Experiment C (SS316L) - Topographical process map at $100 \mu \mathrm{m}$ 


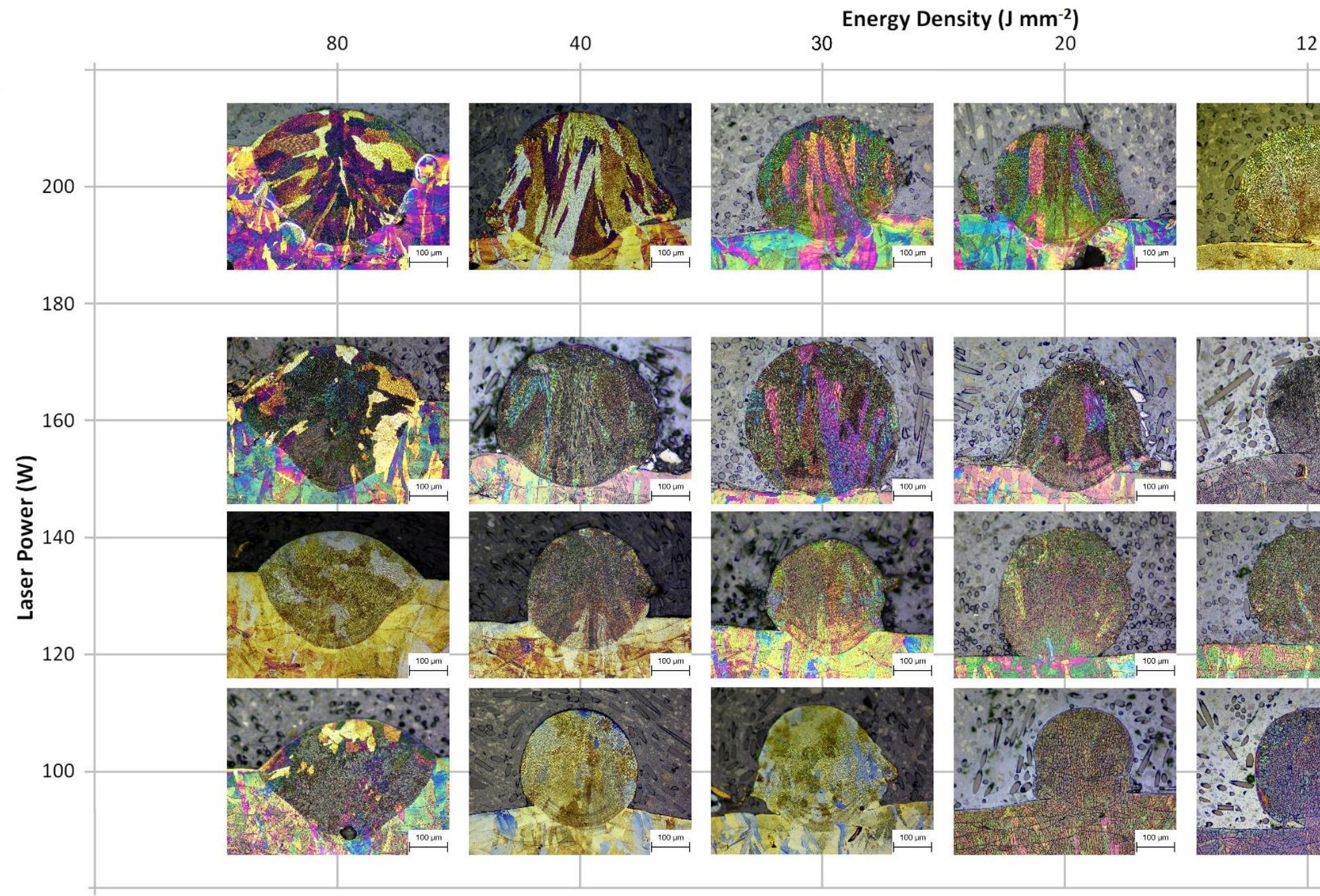

$\stackrel{\text { Increasing Scan Speed }\left(\mathrm{mm} \mathrm{s}^{-1}\right)}{\longrightarrow}$

Figure 44. Experiment C (SS316L) - Cross-sectional process map at $100 \mu \mathrm{m}$ 


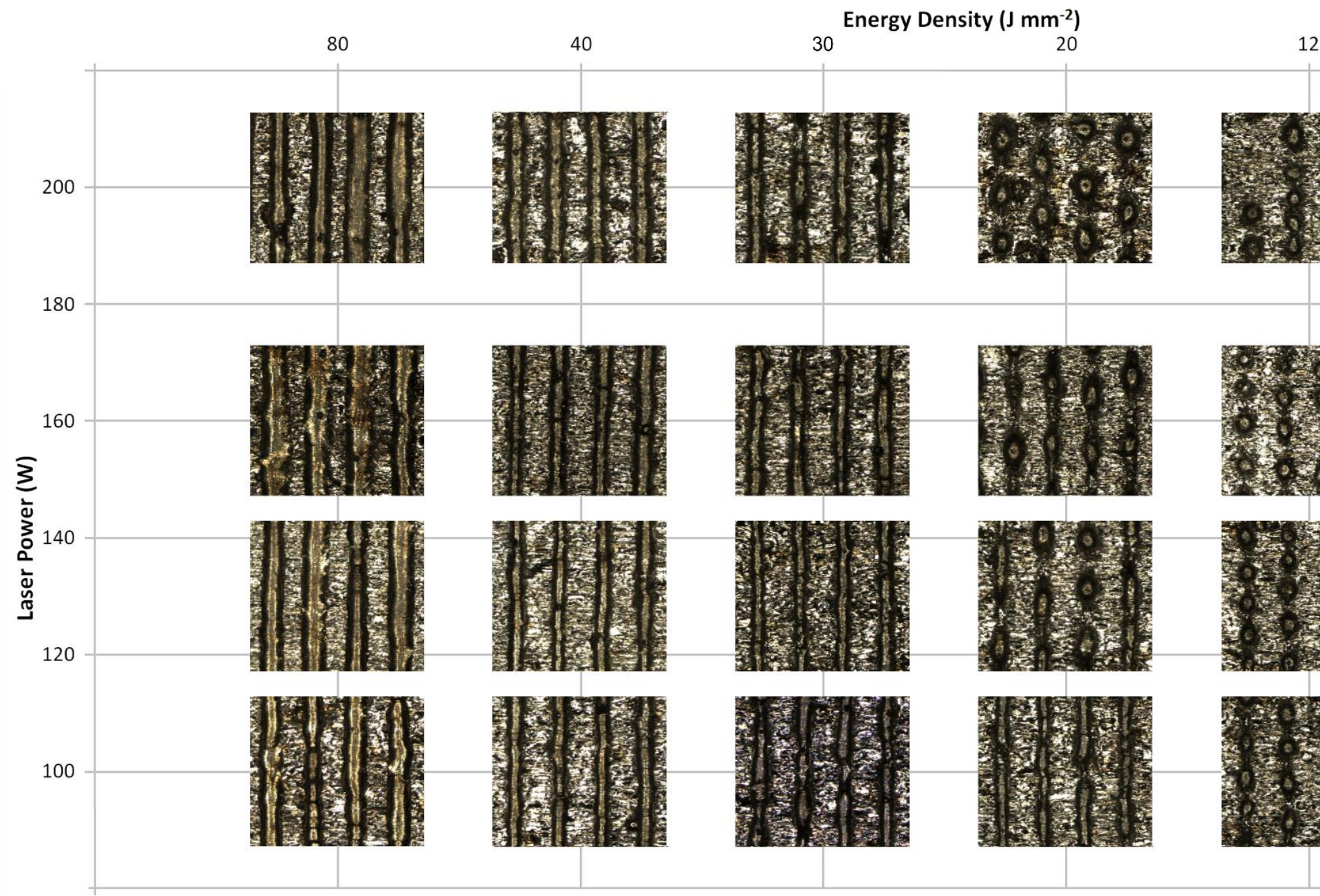

$\stackrel{\text { Increasing Scan Speed }\left(\mathrm{mm} \mathrm{s}^{-1}\right)}{\longrightarrow}$

Figure 45. Experiment C (SS316L) - Topographical process map at $150 \mu \mathrm{m}$ 


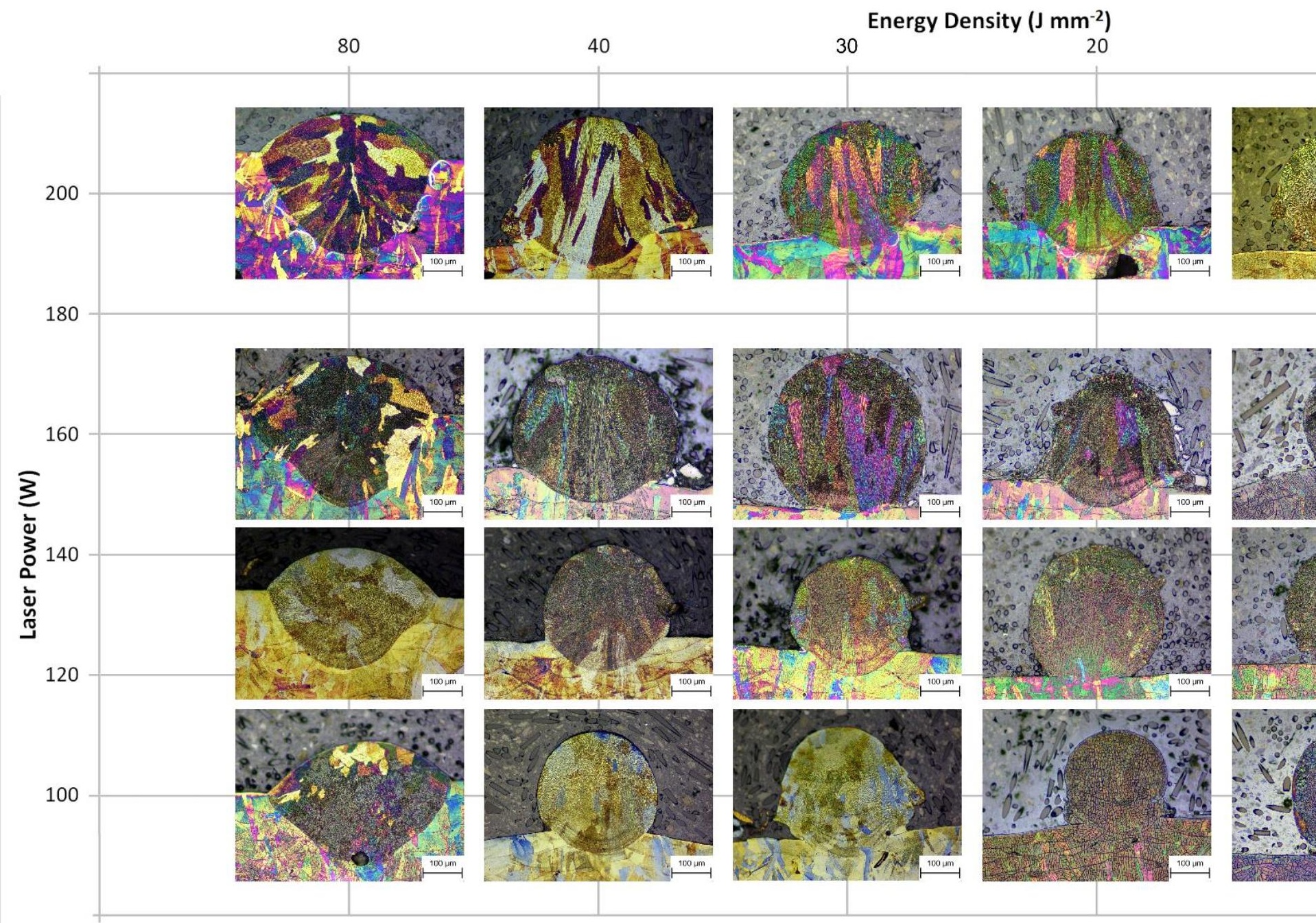

Increasing Scan Speed $\left(\mathrm{mm} \mathrm{s}^{-1}\right)$

Figure 46. Experiment C (SS316L) - Cross-sectional process map at $150 \mu \mathrm{m}$ 


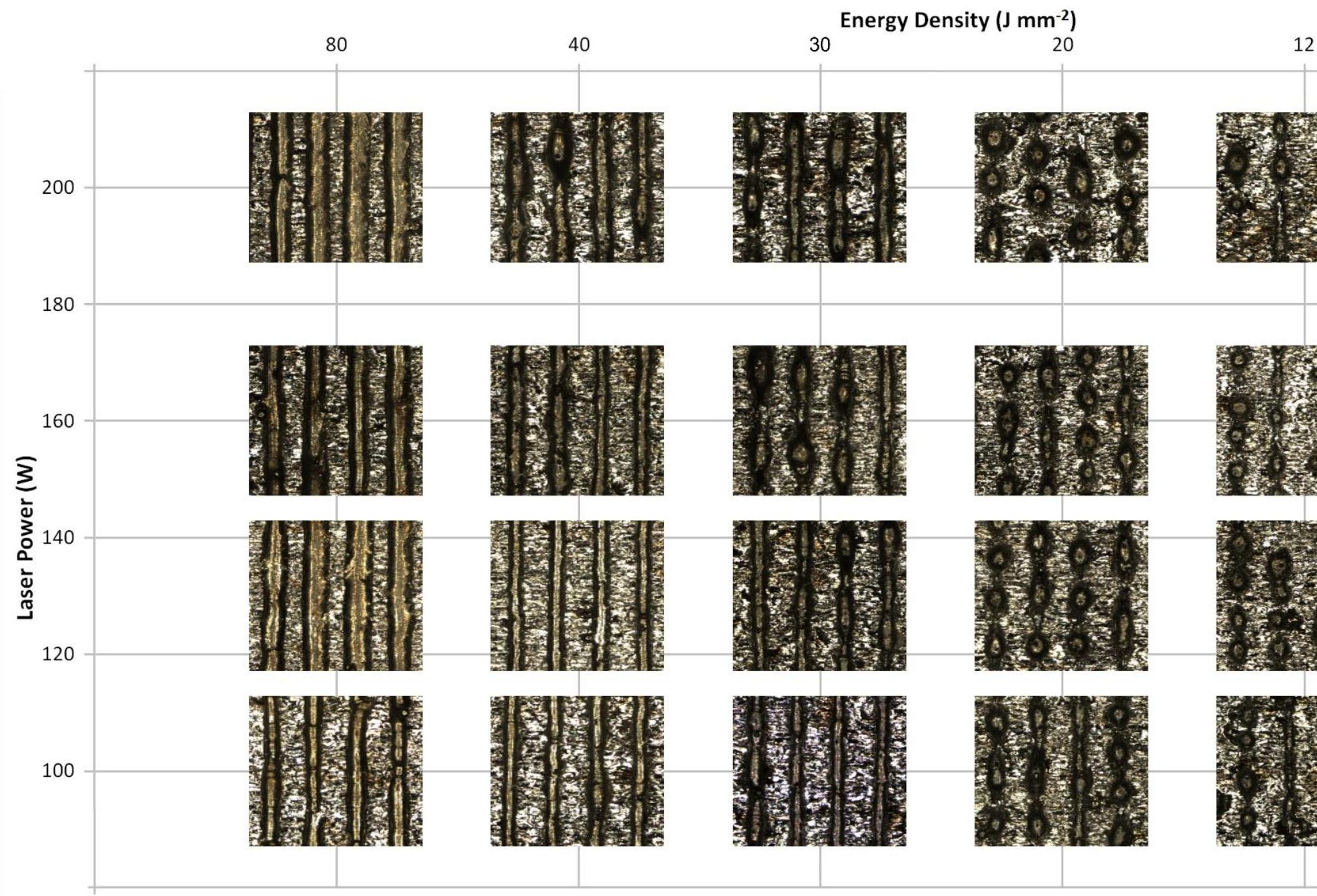

$\stackrel{\text { Increasing Scan Speed }\left(\mathrm{mm} \mathrm{s}^{-1}\right)}{\longrightarrow}$

Figure 47. Experiment C (SS316L) - Topographical process map at $200 \mu \mathrm{m}$ 


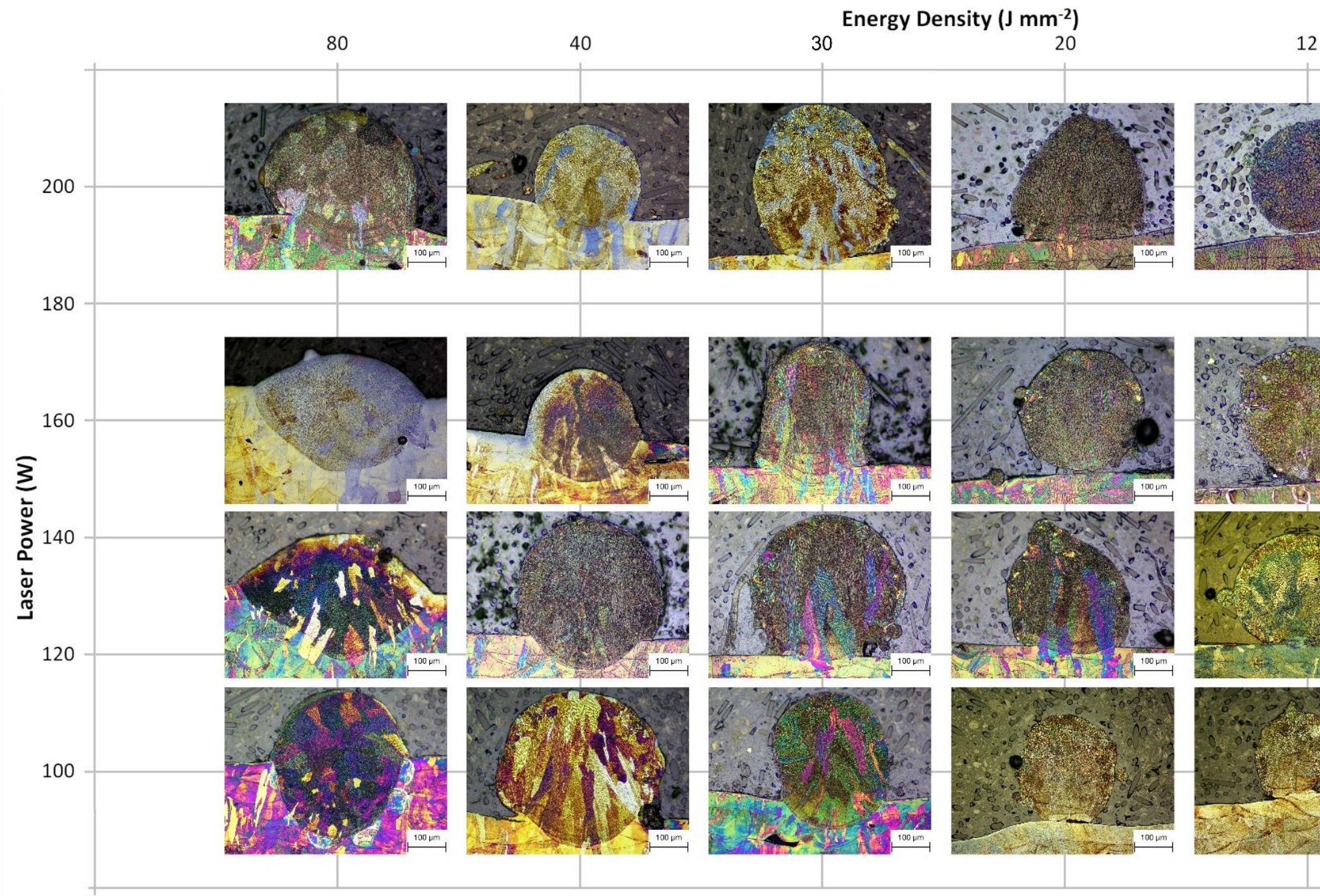

Increasing Scan Speed $\left(\mathrm{mm} \mathrm{s}^{-1}\right)$

Figure 48. Experiment C (SS316L) - Cross-sectional process map at $200 \mu \mathrm{m}$ 


\begin{tabular}{|c|c|c|c|c|c|c|c|c|}
\hline & \multicolumn{8}{|c|}{$50 \mu \mathrm{m}$} \\
\hline \multirow{4}{*}{ 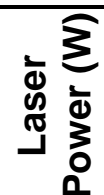 } & $200 W$ & 100 & 100 & 100 & 93.6 & 82.5 & 59.2 & 31.8 \\
\hline & $160 \mathrm{~W}$ & 100 & 100 & 100 & 100 & 87.8 & 74.9 & 20 \\
\hline & $130 \mathrm{~W}$ & 100 & 100 & 100 & 100 & 99 & 89 & 34 \\
\hline & $100 \mathrm{~W}$ & 100 & 99.1 & 96.2 & 96.9 & 92.5 & 67.6 & 51.9 \\
\hline
\end{tabular}

\begin{tabular}{|c|c|c|c|c|c|c|c|c|}
\hline & \multicolumn{8}{|c|}{$100 \mu \mathrm{m}$} \\
\hline \multirow{4}{*}{ 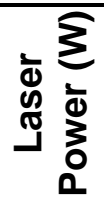 } & $200 W$ & 100 & 100 & 99 & 70.1 & 79.9 & 47.3 & 12.8 \\
\hline & $160 \mathrm{~W}$ & 100 & 100 & 100 & 98 & 66.6 & 48.3 & 0 \\
\hline & $130 \mathrm{~W}$ & 100 & 100 & 100 & 95 & 68.6 & 67 & 0 \\
\hline & $100 \mathrm{~W}$ & 100 & 98.1 & 94.7 & 94 & 72.9 & 50.3 & 15.5 \\
\hline
\end{tabular}

\begin{tabular}{|c|c|c|c|c|c|c|c|c|}
\hline & \multicolumn{8}{|c|}{$150 \mu \mathrm{m}$} \\
\hline \multirow{4}{*}{ 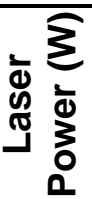 } & $200 W$ & 100 & 100 & 93.2 & 68.4 & 55.9 & 15.4 & 0 \\
\hline & $160 W$ & 100 & 100 & 100 & 89.2 & 58.8 & 23.2 & 0 \\
\hline & $130 \mathrm{~W}$ & 100 & 100 & 100 & 82.8 & 59.4 & 50.1 & 0 \\
\hline & $100 W$ & 98 & 98.5 & 87.6 & 89.3 & 69.3 & 41.7 & 0 \\
\hline
\end{tabular}

\begin{tabular}{|c|c|c|c|c|c|c|c|c|}
\hline & \multicolumn{8}{|c|}{$200 \mu \mathrm{m}$} \\
\hline \multirow{4}{*}{ 㐫 } & $200 W$ & 100 & 89.7 & 92 & 61.2 & 42.9 & 0 & $\overline{0}$ \\
\hline & 160W & 100 & 100 & 97 & 76.3 & 42.1 & 2.4 & 0 \\
\hline & $130 W$ & 100 & 100 & 96 & 72.6 & 51.1 & 23.2 & $\overline{0}$ \\
\hline & $100 \mathrm{~W}$ & 98 & 98.4 & 87 & 65.2 & 55.9 & 17.8 & $\underline{0}$ \\
\hline & & 80 & 40 & 30 & 20 & 12 & 8 & 4 \\
\hline & & \multicolumn{7}{|c|}{ Surface Energy Density $\left(\mathrm{Jmm}^{-2}\right)$} \\
\hline
\end{tabular}

Table 15. Experiment C (SS316L) - Process maps with line build percentages for each layer depth, given in bold over each table.

\subsubsection{Discussion}

Using the DOE parameters mentioned in Chapter 5.1.2 at a layer depth of $50 \mu \mathrm{m}$ resulted in low line build percentages, between $82.5 \%$ and $59.2 \%$. Line fragmentation and balling was prominent, which is usually associated with causing detrimental properties within parts.

Tracks formed with similar processing parameters in Experiment B formed irregularly, but as continuous, unfragmented tracks with a line build percentage above $90 \%$. This difference could be due to the difference in substrates used, and will be further discussed in detail in the results and discussion for Experiment $\mathrm{E}$.

For each layer depth, three distinct types of track formations were identified. Their typical cross-sectional appearance can be seen in Figure 49. 

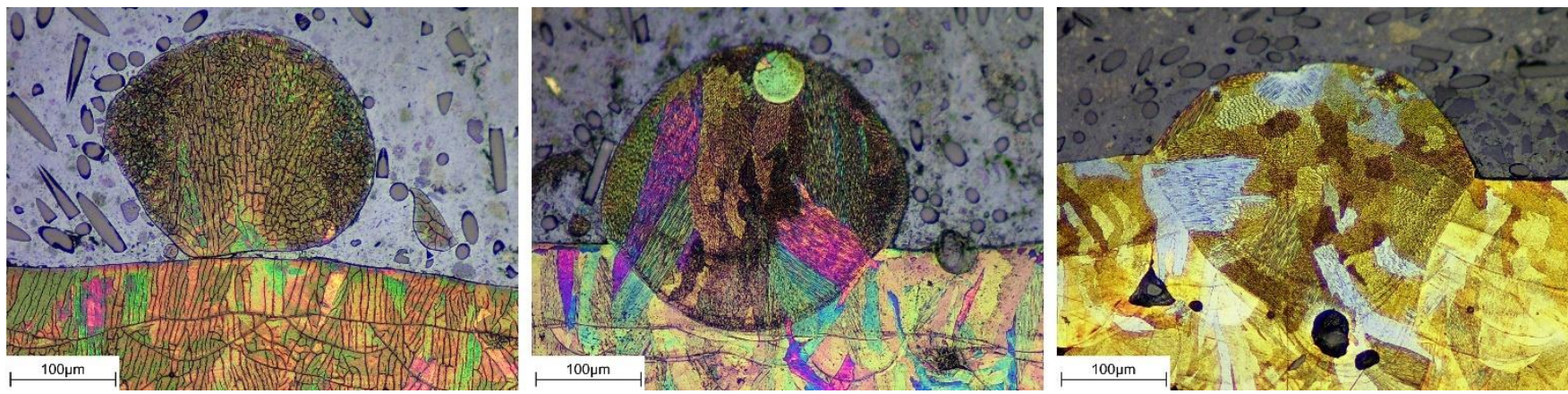

Figure 49. Experiment C (SS316L) - The three types of tracks formed. Left) Balling at $130 \mathrm{~W}, 4 \mathrm{Jmm}^{-2}, 844 \mathrm{mms}^{-1}$ Middle) Rounded top at $160 \mathrm{~W}, 20 \mathrm{Jmm}^{-2}$, $169 \mathrm{mms}^{-1}$ Right) Large track with V-shaped penetration $100 \mathrm{~W}, 30 \mathrm{Jmm}^{-2}, 130 \mathrm{mms}^{-1}$. Each formed at $50 \mu \mathrm{m}$ layer thickness.

\section{Flattened Tracks and Keyhole Mode Melting}

The most distinctive and unique form of track formation were the flattened, continuous

tracks, as observed in Figure 50.The uppermost surface of the melt bead was observed to

be level with the surface of the substrate, and the bead was seen to be completely

submerged within the substrate. The resolidified bead had an ellipsoidal shape, and a

distinct ridge at the bottom of the bead, as seen on the right-hand image of Figure 50 . In

addition, some of these structures would have a clearly visible pore at the bottom of the melt pool, as seen in the right-hand image of Figure 49.
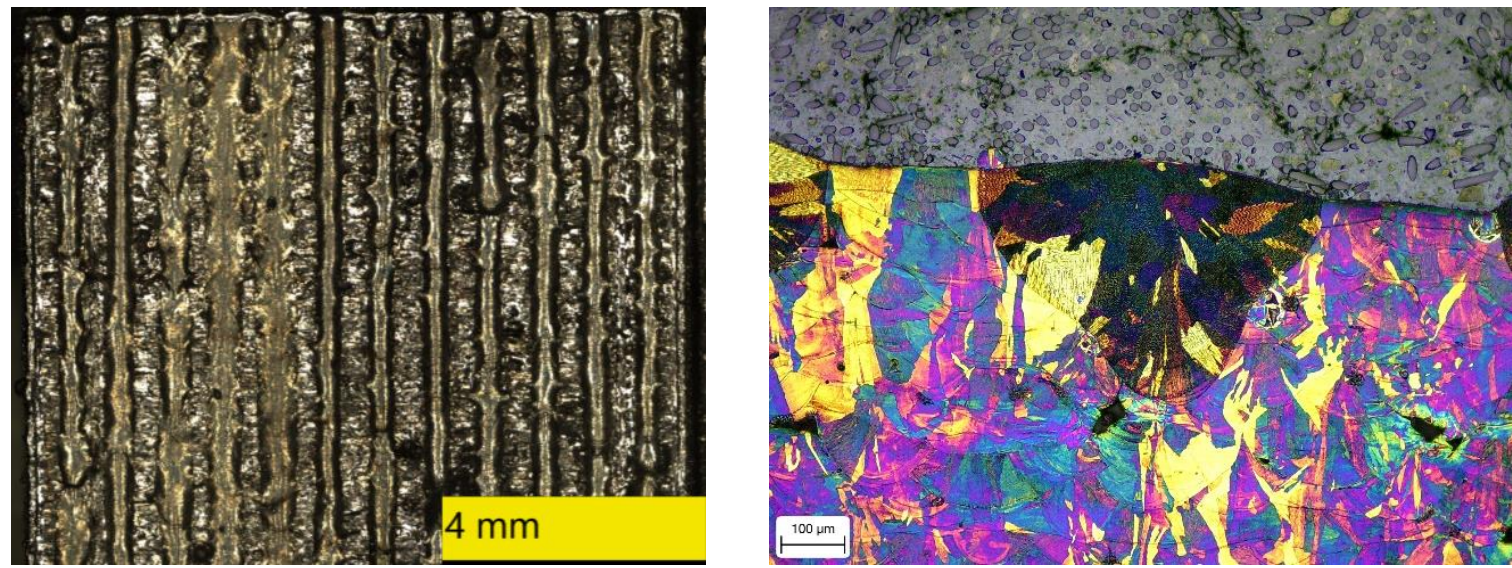

Figure 50. Experiment C (SS316L) - Left) Tracks build on the crucible substrate, with distinctive, flattened tracks appearing on the left-hand side. Right) Crosssectional image taken using the same parameters.

Built at $200 \mathrm{~W}, 80 \mathrm{Jmm}^{-2}, 65 \mathrm{mms}^{-1}$ and at layer depth of $50 \mu \mathrm{m}$.

This distinctive track formation was observed to occur at the regions of highest surface energy density, at $80 \mathrm{Jmm}^{-2}$, at relatively low layer depths of $50 \mu \mathrm{m}$ and $100 \mu \mathrm{m}$. Continuous 
un-flattened tracks would form alongside these flattened tracks, even though the same processing parameters were used to create them, as seen on the left-hand image of Figure 50. The only exception was at one particular combination of processing parameters, at $100 \mathrm{~W}, 32 \mathrm{mms}^{-1}, 80 \mathrm{Jmm}^{-2}$ and a layer depth of $50 \mu \mathrm{m}$, where nearly all the formed tracks exhibited these features.

From the cross-sectional analysis, it was observed that the characteristics of these melt pools resembled those formed by keyhole welding processes, [111], [112]. The scan speeds used during laser welding are considerably low, whilst the laser power is relatively high. A common rule of thumb for welding structural steels is that $1 \mathrm{~kW}$ of power requires a rate of around $17 \mathrm{mms}^{-1}$ to penetrate $1.5 \mathrm{~mm}$ of material, [113]. Whilst the laser power only ranged between $200 \mathrm{~W}$ and $100 \mathrm{~W}$ in these experiments, the thickness of the affected material was comparatively small, at around $50 \mu \mathrm{m}$ to $100 \mu \mathrm{m}$. The speeds at which flattened formations would form ranged from $32 \mathrm{mms}^{-1}$ to $65 \mathrm{mms}^{-1}$. The slow speed of the laser meant that the exposure time was quite high, ranging between 1.5s and 3s. At such conditions, it was assumed that keyhole mode melting takes effect. This was further evidenced by the presence of voids at the bottom edge of the V-shaped penetrations, as explained in Experiment B.

Keyhole mode melting occurs due to the material reaching boiling temperatures rapidly due to the extremely high temperatures. Plasma, ionised vapour, and plume, vapourised material, form within the melt, causing a narrow, deeply penetrating void to form, called a keyhole. The keyhole is maintained through equilibrium of the forces arising from the combined effect of material vapourisation and plasma formation, and hydrostatic pressure and surface tension, the forces of the melt that act to close the void. During keyhole formation, solid material either at the edges of the melt pool or from the remelted layers of the substrate flows around the keyhole cavity and is driven towards the edges of the melt pool by Marangoni flow. Figure 51 displays the mechanisms in affect during keyhole mode melting. As the fluid is spread to the edges, it causes the nearby solid material, whether powder material or previous layers from the substrate, to reach their melting point, and the 
volume of the melt pool increases. This widens the melt pool considerably, and at these processing parameters the width of the melt pools at these parameters exceeded the beam diameter by around 7 to 8 times.

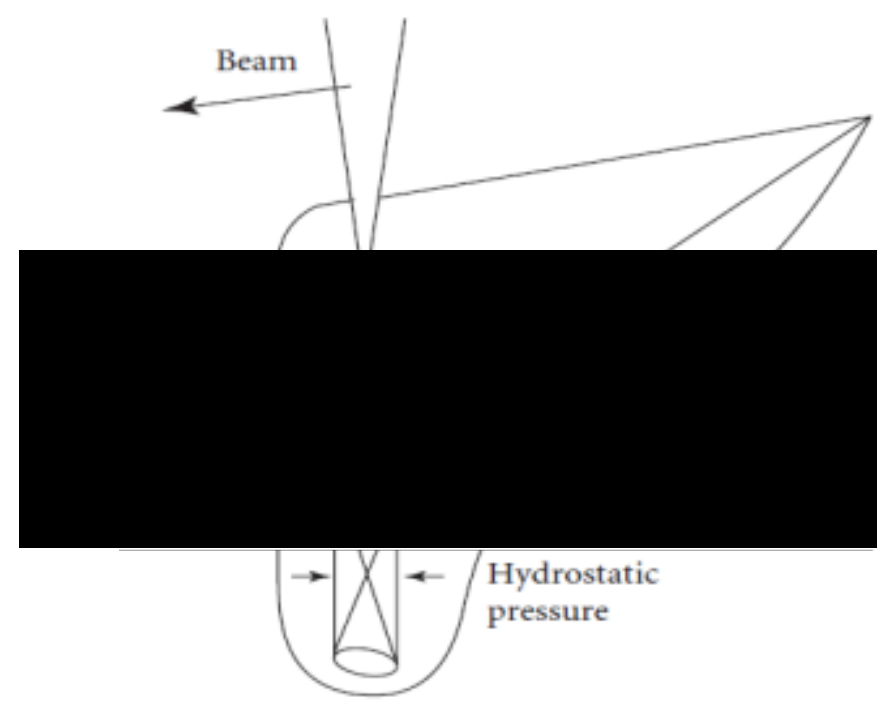

Figure 51. Keyhole formation and fluid flow in the melt pool, taken from Stanciu et al. [113]

When the laser beam moves to the next point, several processes occur. The plasma inside the keyhole is extinguished, the vaporization pressure decays, and the keyhole collapses through the effects of surface tension and gravity. The void forms at the bottom of the melt pool, as described in Chapter 3.

\section{Continuous Regular Tracks}

The second type of track formation were continuous tracks, similar to those seen in Experiment B. The melt profile would have an ellipsoidal shape, with half or more of the bead volume penetrated into the substrate as seen in the middle image of Figure 49. At $50 \mu \mathrm{m}$ layer depth, tracks would build in such a manner between the ranges of $80 \mathrm{Jmm}^{-2}$ and 20 $\mathrm{Jmm}^{-2}$. The range of surface energy densities at which such tracks would be built would narrow slightly with increasing powder depth, ranging between $80 \mathrm{Jmm}^{-2}$ and $30 \mathrm{Jmm}^{-2}$ for layer depths of $100 \mu \mathrm{m}$ and above. Whilst these tracks were continuous, with line build rates 
of around $98 \%$ or higher, as seen in Table 15 , many would exhibit irregularities and distortions. A general observation was that as layer thickness was increased, the height and width of the tracks formed would increase due to the increase in powder volume.

For some of these tracks, whilst the topographical images would show relatively smooth, continuous tracks, keyhole pore formation was observed at every layer thickness used, and would usually occur at higher surface energy density ranges, between $80 \mathrm{Jmm}^{-2}$ and $30 \mathrm{Jmm}$ ${ }^{2}$. Whilst these pores formed in the same manner as described in the previous section, an amount of the melt bead would still be visible over the substrate surface, and tracks would be visibly unflattened. The scan speeds used in these instances was slow enough to cause vapourisation of the melt, but fast enough as not to cause excessive melting of the substrate.

\section{Balling}

Discontinuous, balled tracks would form at regions of low surface energy density. At $50 \mu \mathrm{m}$ layer depth, the tracks that formed between the energy densities of $12 \mathrm{Jmm}^{-2}$ and $4 \mathrm{Jmm}^{-2}$ would break up into a series of spheroidal shapes, as seen in Figure 41. The range of surface energy densities at which balling would occur would increase with layer depth; with $20 \mathrm{Jmm}^{-2}$ and $4 \mathrm{Jmm}^{-2}$ at $100 \mu \mathrm{m}, 20 \mathrm{Jmm}^{-2}$ and $8 \mathrm{Jmm}^{-2}$ at $150 \mu \mathrm{m}$, and $30 \mathrm{Jmm}^{-2}$ and $20 \mathrm{Jmm}^{-2}$ for $200 \mu \mathrm{m}$. Tracks would fail to form at energy densities of $4 \mathrm{Jmm}^{-2}$ for layer depths above $150 \mu \mathrm{m}$, and at $8 \mathrm{Jmm}^{-2}$ or above for $200 \mu \mathrm{m}$. These tracks would form with low line build percentages, as seen in Table 15 , at rates of $82.5 \%$ or lower.

Increasing the layer thickness was seen to increase the balling effect, and lessen the wettability of the melt on the substrate surface. Additionally, the width of the structures was found to increase with layer thickness, due to the increase in powder volume. Increasing the volume of material requires the energy input to increase to compensate for the energy required to melt more material. At these parameter combinations, the energy absorbed by the powder was insufficient, resulting in a relatively low temperature of the melt pool. Due to the low temperature of the melt, the surface tension forces of the melt would be the dominant forming factor in determining its shape, resulting in balling of the melt, poor 
wettability and reduced fluid flow. Additionally, the distance between the melt and the substrate would have increased, leading to reduced contact between them. These two factors result in the formation of isolated droplets instead of continuous tracks, as the reduced wetting area would not be able to support large molten tracks. As volume of the melt would increase with layer depth, balling would become more prominent.

\section{Depth-to-Width Contour Map}

The average width and depth values measured from the tracks created in this experiment were used to make four contour plots of the depth-to-width ratios, as seen in Figure 52 below. It was generally observed that the depth-to-width ratio would increase with surface energy density. The red and orange regions, where depth-to-width ratios were near 0.6 or above, are regions where keyhole mode melting was observed. This was due to either laser power being too high, scan speed being too low, or a combination of both, resulting in vapourisation of the melt. At the green and yellow regions, where depth-to-width ratios ranged between 0.5 and 0.2 , tracks would form as continuous tracks with suitable penetration into the substrate. At light blue or white regions, where depth-to-width ratios ranged between near 0.2 or lower, prominent balling was observed. Penetration depth would be minimal or not even measurable in many cases. 

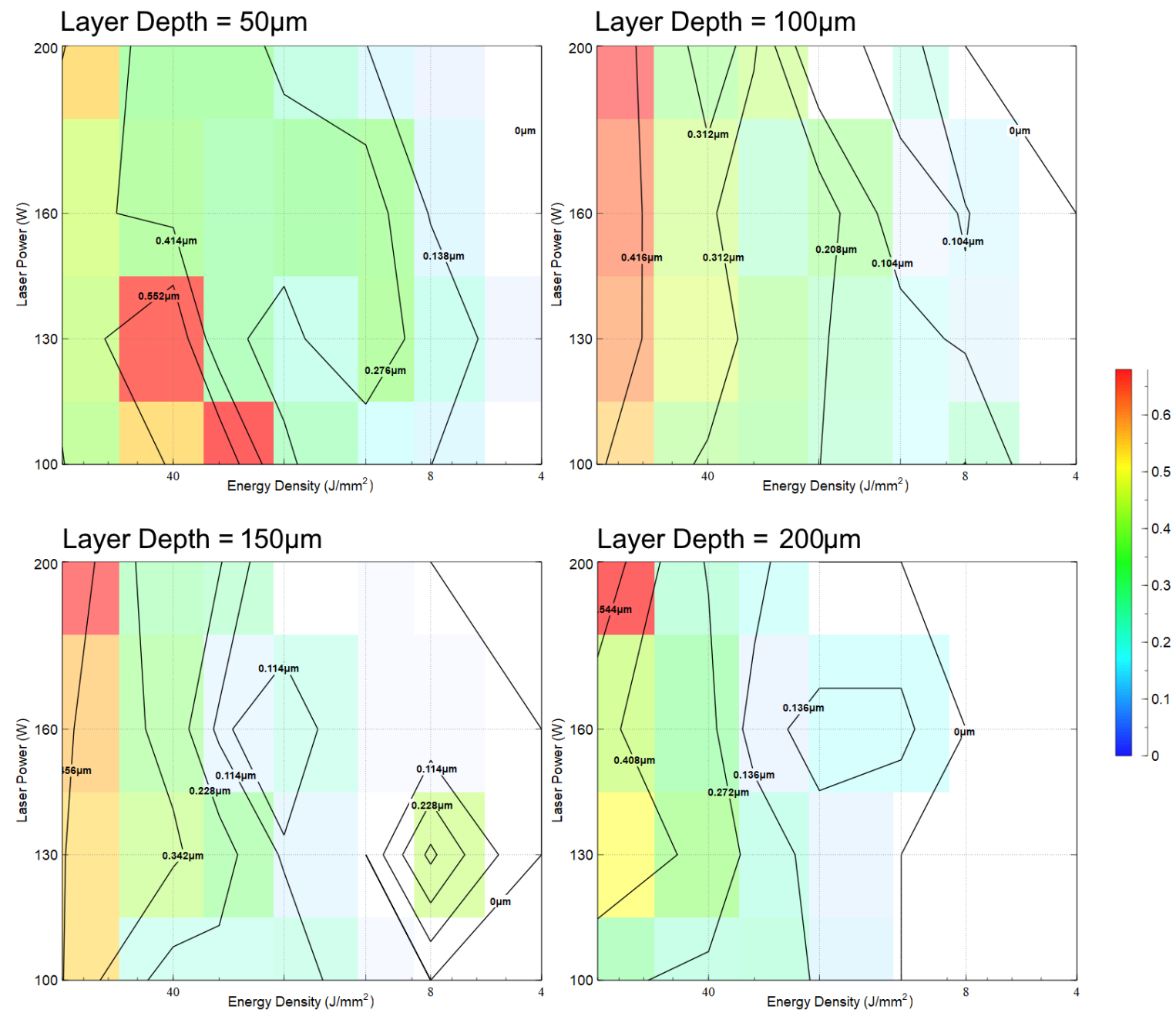

Figure 52. Experiment C (SS316L) - Contours of depth-to-width ratios at the different layer depths.

\subsubsection{Conclusions}

It was found that surface energy density was not a suitable variable for predicting track formation, as tracks built using the same surface energy values with different parameters gave wildly different results to one another.

A distinct form of track formation was observed, which was not observed in any other experiment mentioned in this research. Using large exposure times, ranging between $1.5 \mathrm{~s}$ and 3 s, resulted in excessive heating of the powder and substrate material. This resulted in keyhole mode melting and the elimination of the track height above the substrate as the melt bead would sink below the surface. 
Increasing the layer depth was found to increase the prominence of balling, as increasing the powder volume was found to reduce the wettability between the substrate and the melt. The general trends seen in previous experiments regarding the changes in scan speed and laser power were observed in this experiment as well. The degradation of track stability as scan speed increased was plainly observed in the results.

Tracks were found to build with desirable properties, that is to say, as continuous, regular tracks with no visible pore formation, at many different input parameter combinations for varying layer depth. For each layer depth investigated, the following process parameters were selected for their optimal track formation properties. These parameters were selected as they had formed continuous, smooths tracks with $100 \%$ build rates and low depth-towidth ratios at their specified layer depth:

I. $50 \mu \mathrm{m}$ depth: Laser power of $100 \mathrm{~W}$ and scan speed of $87 \mathrm{mms}^{-1}\left(30 \mathrm{Jmm}^{-2}\right)$.

II. $100 \mu \mathrm{m}$ depth: Laser power of $100 \mathrm{~W}$ and scan speed of $87 \mathrm{mms}^{-1}\left(30 \mathrm{Jmm}^{-2}\right)$.

III. $150 \mu \mathrm{m}$ depth: Laser power of $130 \mathrm{~W}$ and scan speed of $113 \mathrm{mms}^{-1}\left(30 \mathrm{Jmm}^{-2}\right)$

IV. $200 \mu \mathrm{m}$ depth: Laser power of $130 \mathrm{~W}$ and scan speed of $84 \mathrm{mms}^{-1}\left(40 \mathrm{Jmm}^{-2}\right)$. 


\subsection{Experiment D - Single-Tracks on Crucible Substrates}

\subsubsection{Results}

Topographical images of the tracks successfully constructed from experiment $D$ were compiled into a process map as a function of scan speed on the horizontal axis, and laser power on the vertical axis, as seen in Figure 53. Using the same axes as Figure 53, a process map was made using cross-sectional images using the same parameters, as seen in Figure 54. On both figures, the optimal parameter combination used for the DOE in Chapter 5.1.2 was included as a figure in these process maps as a reference to the parameters used to obtain optimal bulk as-built density for this specific batch of powder material.

An additional process map was created using the line build percentages measured from the successfully built tracks, using the same technique mentioned in the experimental design section for experiment B. This process map can be seen in Table 16. Each image comprises three lines, which are a repetition of the tracks using the same power and scan speed. Areas with missing images are regions where the combination of laser power and scan speed caused little or no track formation.

\begin{tabular}{|c|c|c|c|c|c|c|c|c|c|c|c|}
\hline \multirow{6}{*}{ 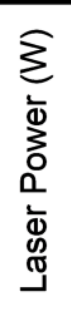 } & 200 & 100.0 & 100.0 & 100.0 & 99.0 & 98.5 & 95.0 & 82.3 & 78.7 & 59.2 & 57.2 \\
\hline & 175 & 100.0 & 100.0 & 100.0 & 98.3 & 92.5 & 77.6 & 68.9 & 61.7 & 41.3 & 40.8 \\
\hline & 150 & 100.0 & 100.0 & 99.0 & 97.9 & 82.9 & 69.9 & 48.7 & 30.7 & 16.5 & 10.7 \\
\hline & 125 & 100.0 & 100.0 & 99.7 & 97.1 & 81.9 & 56.1 & 20.0 & 0.0 & 0.0 & 0.0 \\
\hline & 100 & 100.0 & 99.5 & 98.0 & 73.7 & 54.5 & 25.4 & 0.0 & 0.0 & 0.0 & 0.0 \\
\hline & 75 & 100.0 & 93.5 & 72.8 & 38.7 & 11.0 & 0.0 & 0.0 & 0.0 & 0.0 & 0.0 \\
\hline & & 100 & 200 & 300 & 400 & 500 & 600 & 700 & 800 & 900 & 1000 \\
\hline & & & & & & $\overline{\mathrm{nS}}$ & $\overline{\mathrm{mn}}$ & & & & \\
\hline
\end{tabular}

Table 16. Experiment D (SS316L) - Process map with line build percentages. 


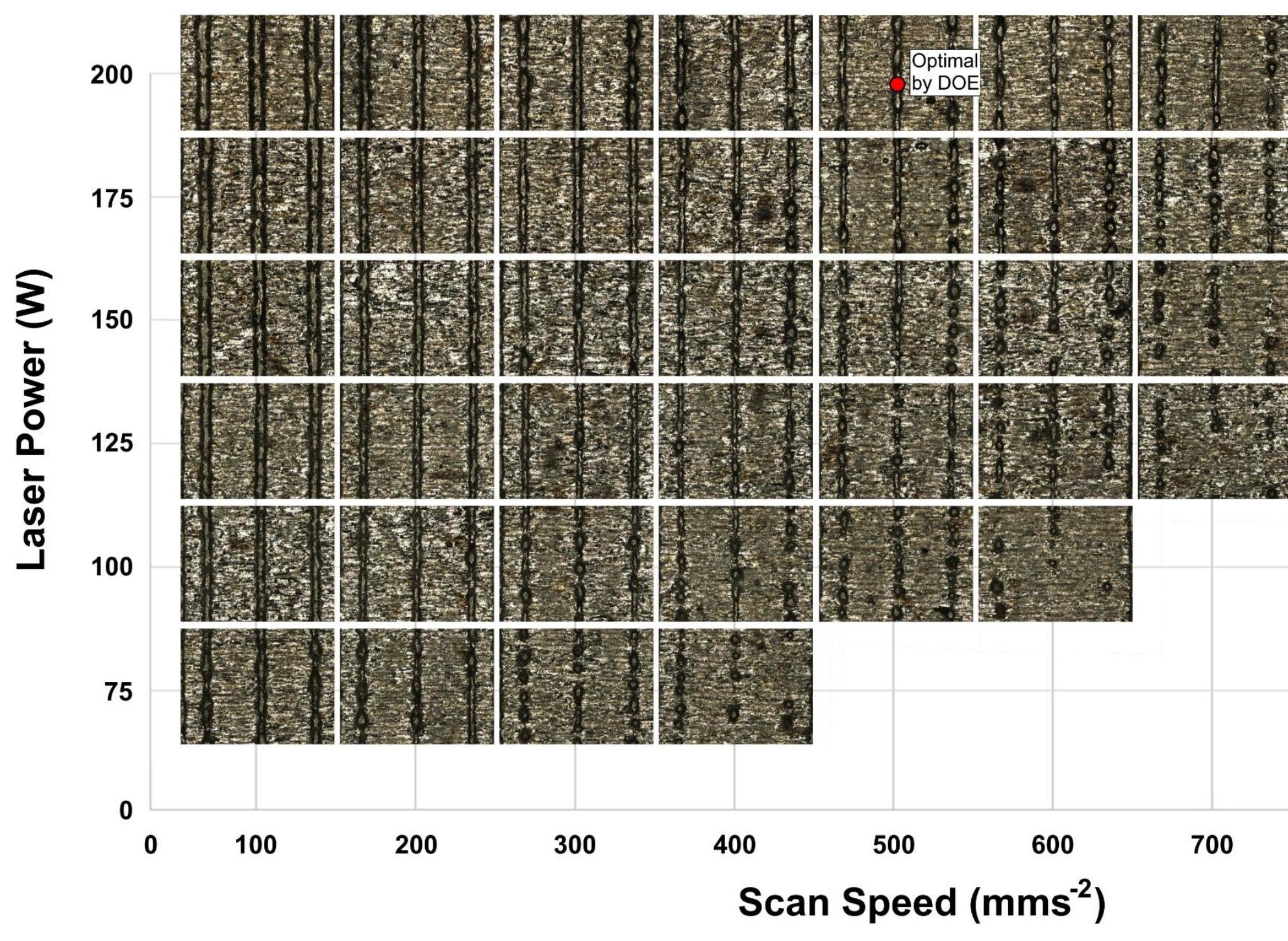

Figure 53. Experiment D (SS316L) - Topographical process map 


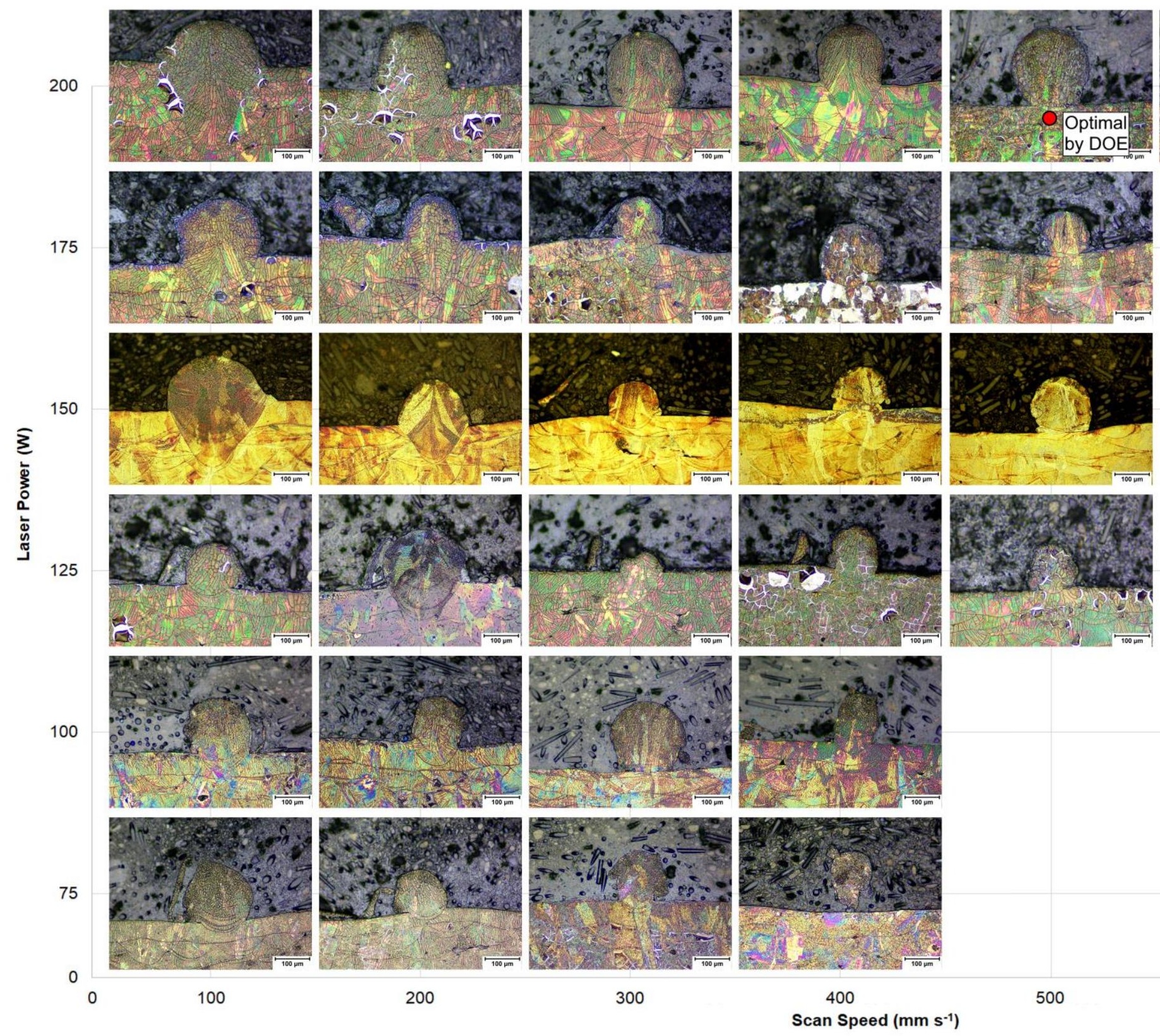

Figure 54. Experiment D (SS316L) - Cross-sectional process map 


\subsubsection{Discussion}

\section{Track Formation and Width Comparison}

In direct comparison to the results from Experiment $B$, the range in which continuous and regular tracks was reduced by one parameter combination, at the lower laser power setting at $150 \mathrm{~W}$ and $100 \mathrm{mms}^{-1}$. Tracks built within this region had a $100 \%-$ line build rate, and are annotated as the blue sections in Table 16. Conversely, the range at which continuous, irregular tracks could form had increased. At laser power values between 175W and 100W, the range by which tracks with near- $100 \%$ could be fabricated had increased by $100 \mathrm{mms}^{-1}$. Additionally, the range in which track could be built with $100 \%$-line build rate within this region had also increased. This region is highlighted in green in Table 16. This trend was observed for the other two types of track formations, including the irregular/discontinuous tracks and balled tracks, highlighted in yellow and orange in Table 16.

The range of process parameters at which track formation could occur had also increased, though the formations were only weakly dispersed balled droplets. In the regions where track failed to build entirely on the insert substrates in Experiment B, such as at 150W and $1000 \mathrm{mms}^{-1}$, track formation had occurred, although it was minimal (10.7\%).

The results from the width measurements from experiment $B$ were compared to the results from the following experiment. The comparison between the width values can be seen in Figure 55 and Figure 56 below.

The average track widths measured from samples built using crucible substrates in experiment $D$ were found to be much larger than those measured from their counterparts in experiment $B$, which were constructed using mild steel inserts. Most of the tracks built using the exact same processing parameters were found to be substantially larger when built on crucibles, except for the values at $175 \mathrm{~W}$, where both sets of measurements gave results similar to one another. 


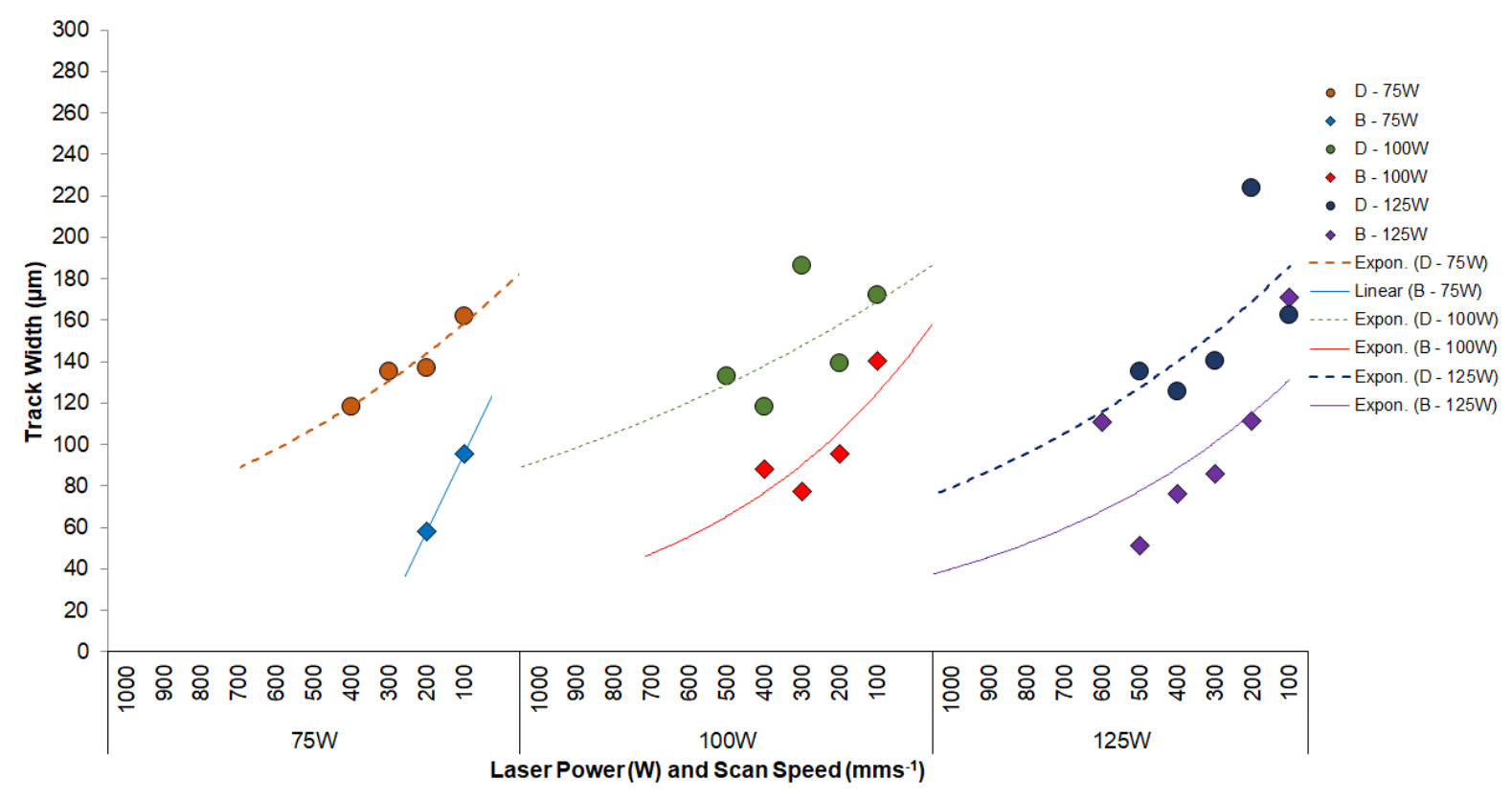

Figure 55. Experiment D (SS316L) - Track widths compared with Experiment B (SS316L) at the 75-125W range.

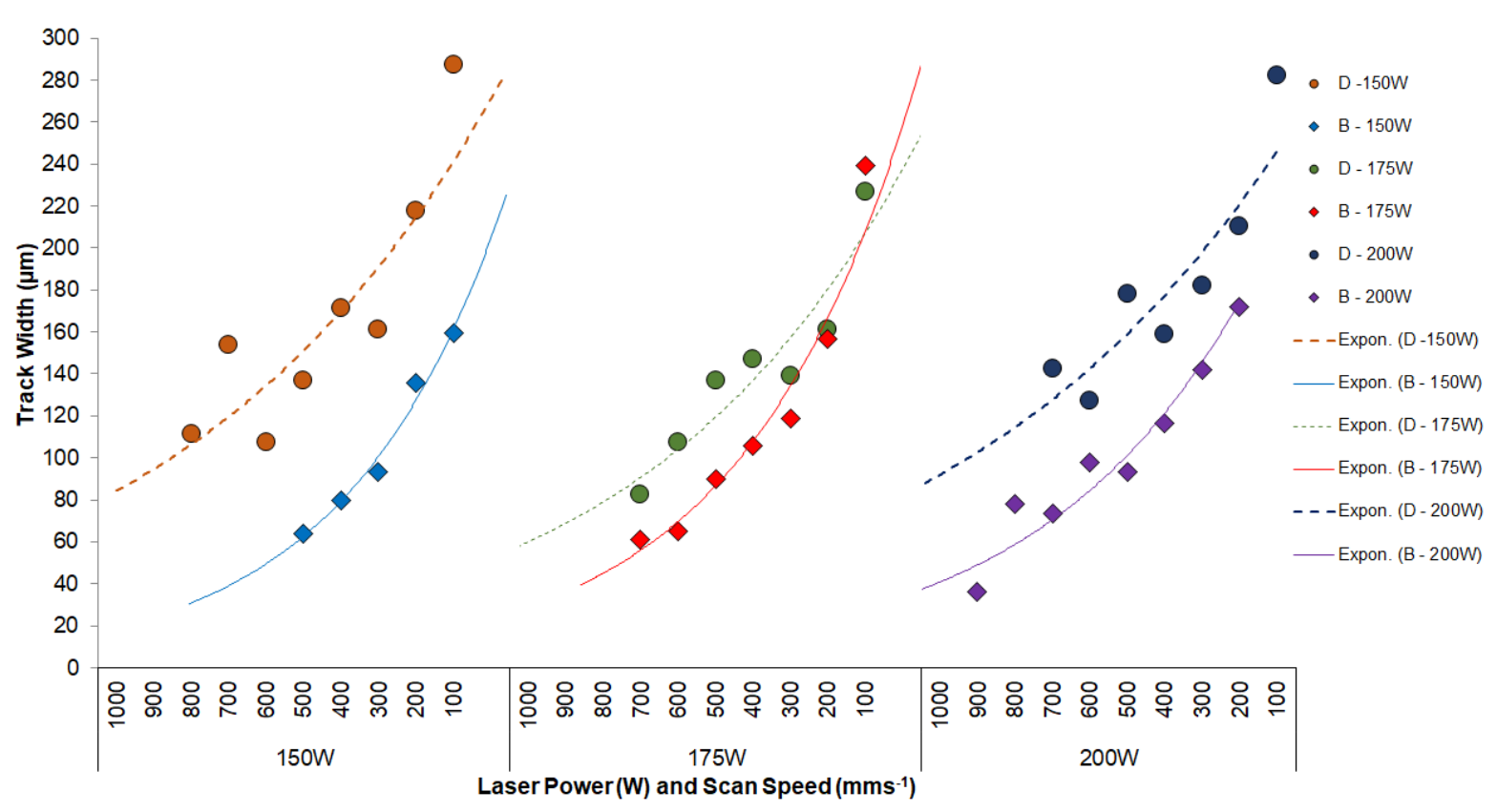

Figure 56. Experiment D (SS316L) - Track widths compared with Experiment $B$ (SS316L) at the 150-200W range. 
The comparison between the results of Experiment $B$ and Experiment $D$ show that track formation was more favoured in Experiment D. Additionally, the width of the structures observed was seen to increase. The only real difference between the two experiments is the type of substrate used. Experiment B used a mild steel plate with a planar surface i.e. flat, smooth surface. Experiment D used the crucible substrate of stainless steel $316 \mathrm{~L}$, made using the laser-fusion additive manufacturing method, using the recommended parameters by the manufacturer.

One of the factors that can influence balling is the presence of oxide contamination. As the steel inserts were prepared in an oxygen environment, a certain degree of oxidation was expected to occur. As a consequence, wetting between the melt and oxide layer on the surface is reduced, [114]. As the crucible substrate was constructed in an environment relatively free of oxygen, the surface could have had a reduced oxide presence, resulting in better wetting properties between the melt and surface and decreased track build failure. The final layer to be printed for the crucible, that is to say, the surface of the substrate that the tracks would build upon, had perpendicular scanning pattern in relation to the track formation. This layer, and the other layers that built the bulk of the crucible, were built parallel to the build plate, with a sloping angle of $0^{\circ}$. A horizontal surface made via laserpowder bed fusion usually has the least surface roughness at this sloping angle, [115]. However, as seen in Experiment A, laser-powder bed fusion still tends to create surfaces with rippled, wave-like patterns, and as a result these surfaces would have a higher surface roughness than machined and polished surfaces. Increasing surface roughness affects the wettability of liquids in contact with the surface. The relationship between roughness and wettability was defined by Wenzel, [116], who stated that adding surface roughness will enhance the wettability of the melt, defined by the following equation:

$$
\cos \theta_{m}=r \cos \theta_{Y}
$$

Where $\theta_{m}$ is the measured contact angle, $\theta_{Y}$ is the Young contact angle, that is, the contact angle at a $0^{\circ}$ horizontal surface and $r$ is the roughness ratio, the ratio between the actual and projected solid surface area, with $r=1$ for a completely smooth surface, and $>1$ for a rough 
one. This equation is based on the assumption that liquid penetrates into surface roughness grooves.

Improving the wettability between the liquid and solid interface improves the ability of the tracks to form, as previously discussed. This is evidenced at particularly low settings, where tracks would fail to form on a smooth substrate but managed to form on the rougher crucible substrate. Improving the wettability across the entire range of process parameter combinations would result in an increase in average width for all the structures observed. This is due to the melt being able to spread more easily on its surface, thus coming into contact with more surrounding material and causing it to reach melting temperatures, increasing the volume of the melt. It has been shown in literature that for laser-powder bed fusion the wettability, and by consequence, the width of formed tracks, can increase as the surface roughness of the substrate increases due to these mechanisms, [117], [118].

\section{Cross-Sectional Comparison}

From examination of the cross-sectional images, three forms of solidified track were observed, as seen in Figure 57. In regions of low laser power, $75 \mathrm{~W}$ to $100 \mathrm{~W}$, or high scan speeds, $400 \mathrm{mms}^{-1}$ to $800 \mathrm{mms}^{-1}$, the track solidified into a spherical structure which was loosely attached to the base plate, such as at laser power $200 \mathrm{~W}$, scan speed $700 \mathrm{mms}^{-1}$. This formation corresponded with the balling defect, as discussed previously in Experiments $\mathrm{B}$ and $\mathrm{C}$. The combination of these processing parameters would not achieve the time of temperature required in the melt pool to cause sufficient wetting with the substrate surface. In regions of middle to high laser power, between $125 \mathrm{~W}$ and $200 \mathrm{~W}$, and at low to moderate scan speeds, between $100 \mathrm{mms}^{-1}$ and $400 \mathrm{mms}^{-1}$, tracks would form with a rounded top, with a small or medium elliptical formed penetration depth. The temperature achieved at these setting was suitable for wetting and spreading of the melt, resulting in continuous track formation. At very high laser powers, between $175 \mathrm{~W}$ and $200 \mathrm{~W}$, with low scan speeds, between $100 \mathrm{mms}^{-1}$ and $200 \mathrm{mms}^{-1}$, the track would form with a deeper V-shaped penetration 
below the substrate, as seen in the right-most image in Figure 57. At this range of settings, the energy input was high enough to cause keyhole mode melting.
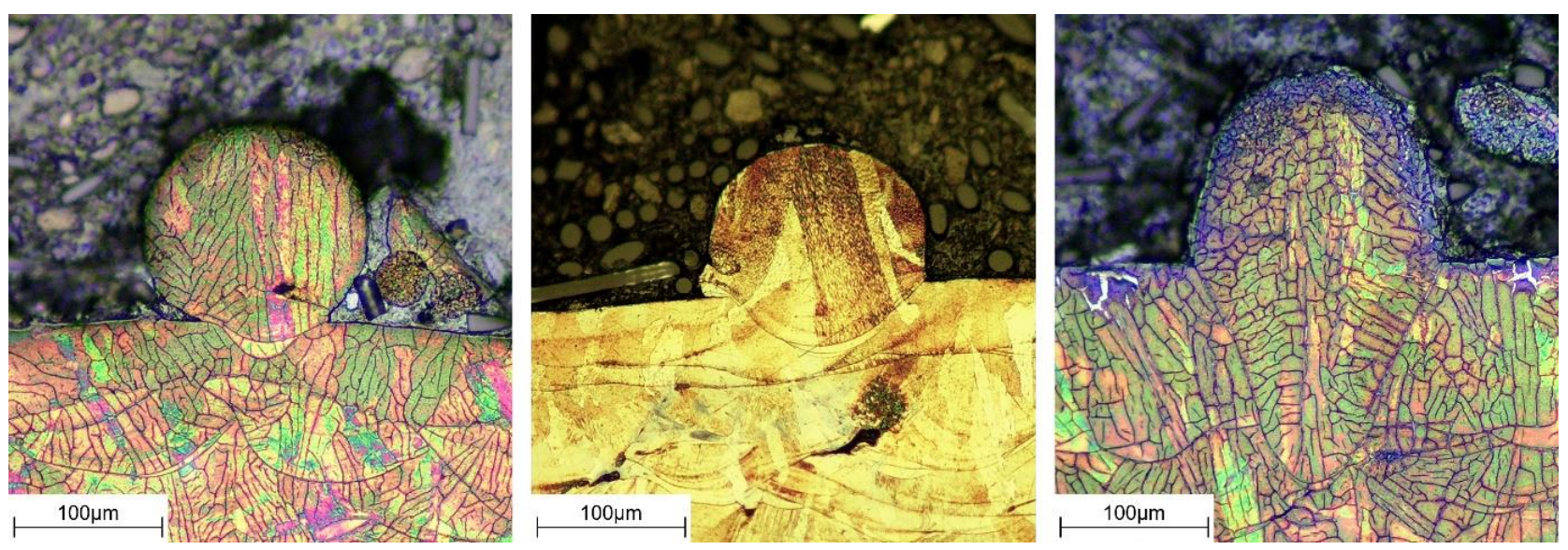

Figure 57. Experiment D (SS316L) - The three types of tracks formed. Left) $200 \mathrm{~W}, 700 \mathrm{mms}^{-1}$, Middle) 150W, $300 \mathrm{mms}^{-1}$, Right) 175W, 200 $\mathrm{mms}^{-1}$.

Keyhole porosity was observed in three instances, and are displayed in Figure 58.In the leftmost and bottom images in Figure 58, the melt pool that formed during the experiment in each instance formed a track with a deep penetration, nearly twice as deep as the track was wide, and a large pore formed at the very tip of the penetration. Conversely, the image on the right of Figure 58 had a track form with a large, cylindrical cross-section, with the track width being larger than the track depth. A small pore was present at the very tip of the penetration. Columnar grain growth within the penetration of the track, i.e. below the substrate surface, can be observed plainly in each cross-section. Smaller, equiaxed grain growth can be observed at the top of the track bead. 

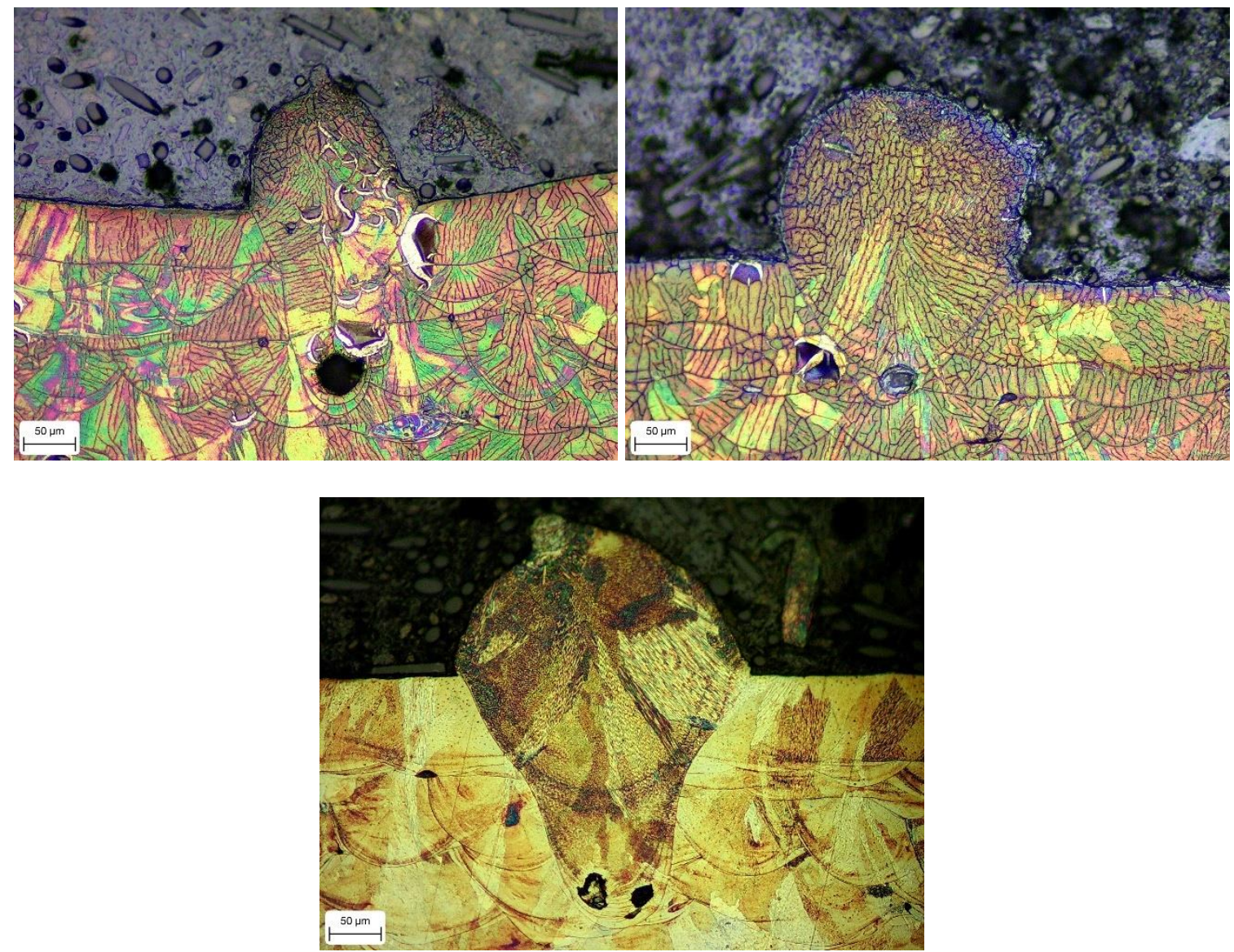

Figure 58. Experiment D (SS316L) - Keyhole porosity observed during experiment. Top left) $200 \mathrm{~W}, 300 \mathrm{mms}^{-1}$, top right) $175 \mathrm{~W}, 100 \mathrm{mms}^{-1}$, bottom) $150 \mathrm{~W}, 100 \mathrm{mms}^{-1}$

\section{Depth Comparison and Verification}

A comparison between the average penetration depths of Experiment $B$ and $D$ can be seen

below in Figure 59 and Figure 60. Measured depths from the crucible samples were usually deeper, although the difference was not as drastic as observed in the changes in average width. This could be attributed to the increase in wetting due to the increase in surface roughness allowing the melt to reach the substrate in a shorter amount of time, and thereby allowing increased melting of the substrate. The depths measured from the tracks made using the insert samples were slightly higher than their crucible counterparts at $175 \mathrm{~W}$. 


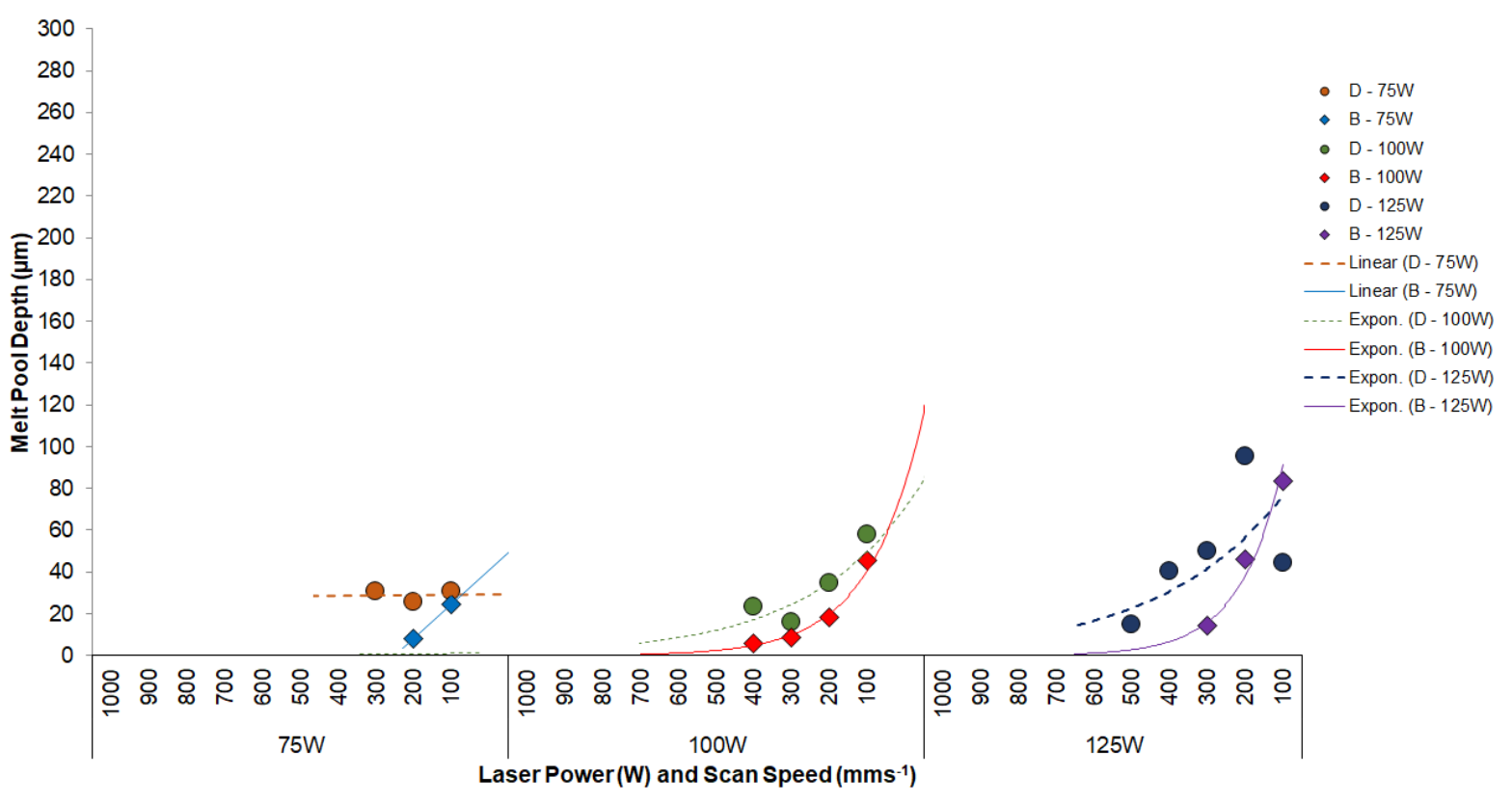

Figure 59. Experiment D (SS316L) - Track depths compared with Experiment $B$ (SS316L) at the 75-125W range.

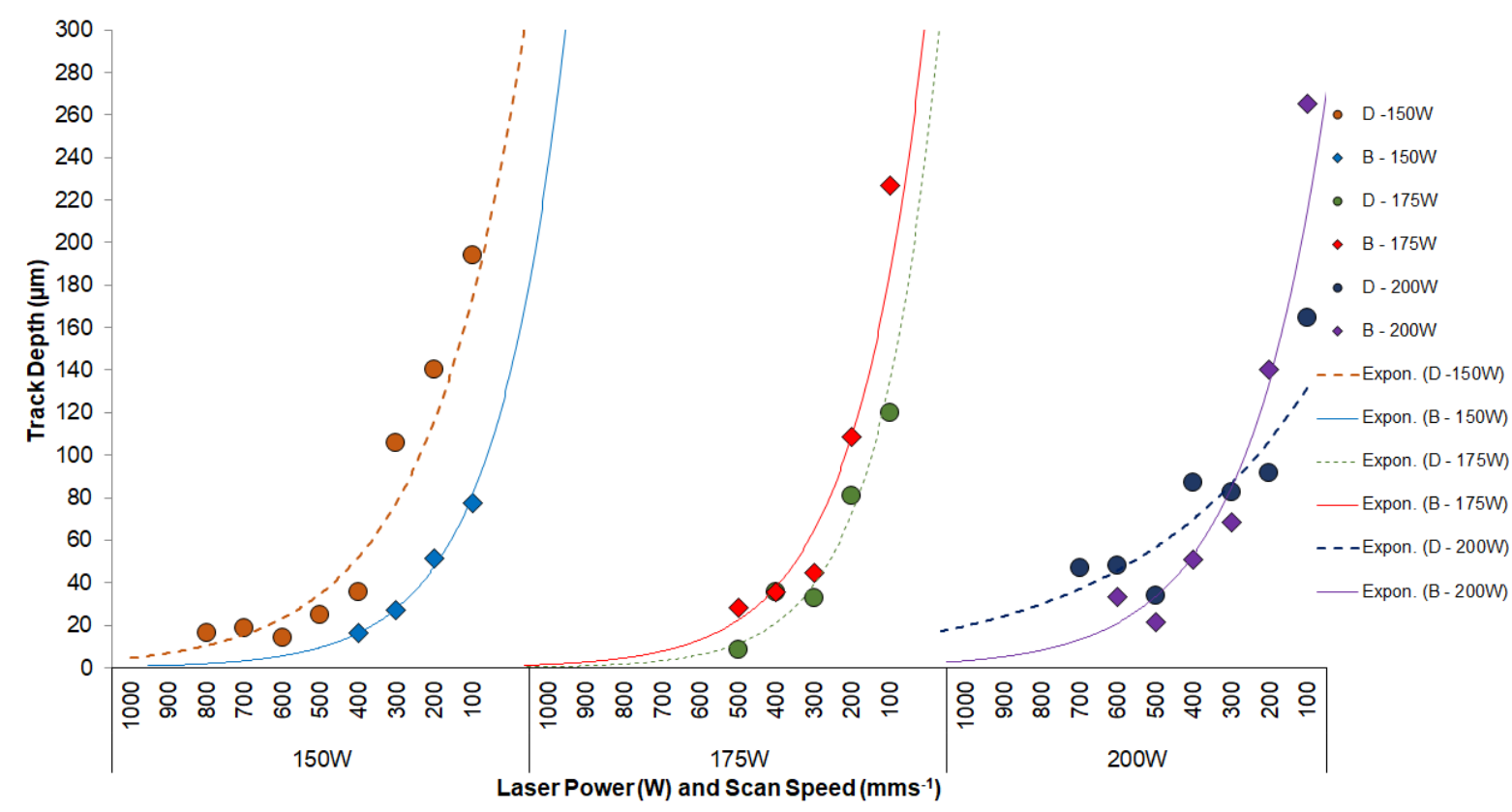

Figure 60. Experiment D (SS316L) - Track depths compared with Experiment B (SS316L) at the 150-200W range.

The depth measurements were also plot against values calculated by the deep penetration melting model used in Experiment B, developed by Gladush and Smurov, [108]. These plots are shown in Figure 61 and Figure 62. 
A good correlation between the predicted values and measured values was observed for most laser power and scan speed combinations, particularly at the lower energy densities. However, at the highest range of laser power settings used, measured values at the lowest scan speeds were found to be exceptionally higher than predicted. At these parameter combinations, keyhole mode melting was observed in many samples.

This is in line with the findings of King et al [119], , who pointed out the limitations of this empirical model at high energy density melting using 400W lasers and small beam diameters, in which there was a higher propensity for keyhole mode melting to occur.

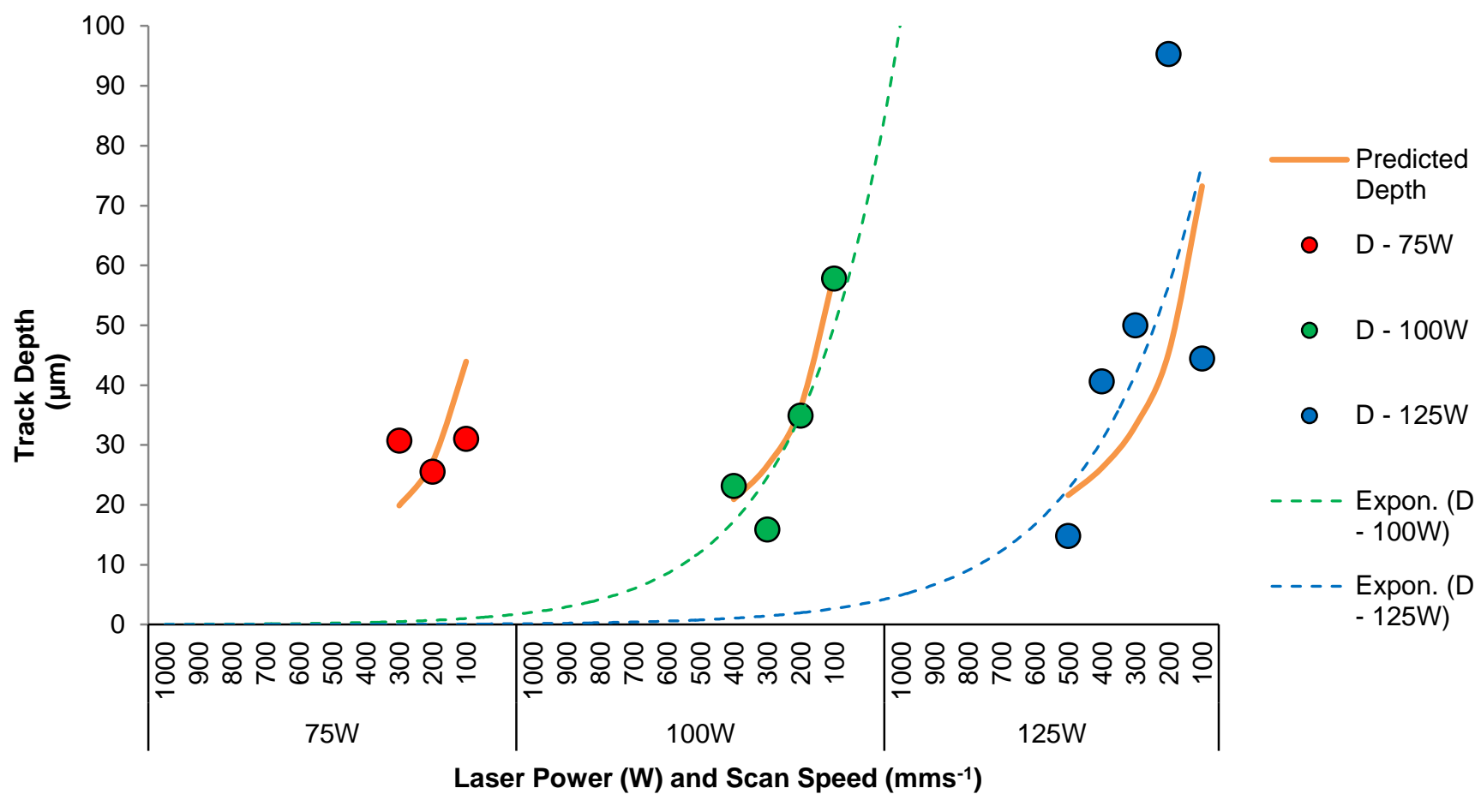

Figure 61. Experiment D (SS316L) - Comparison of measured and predicted penetration depths according to equation from Gladush and Smurov, [108]. 


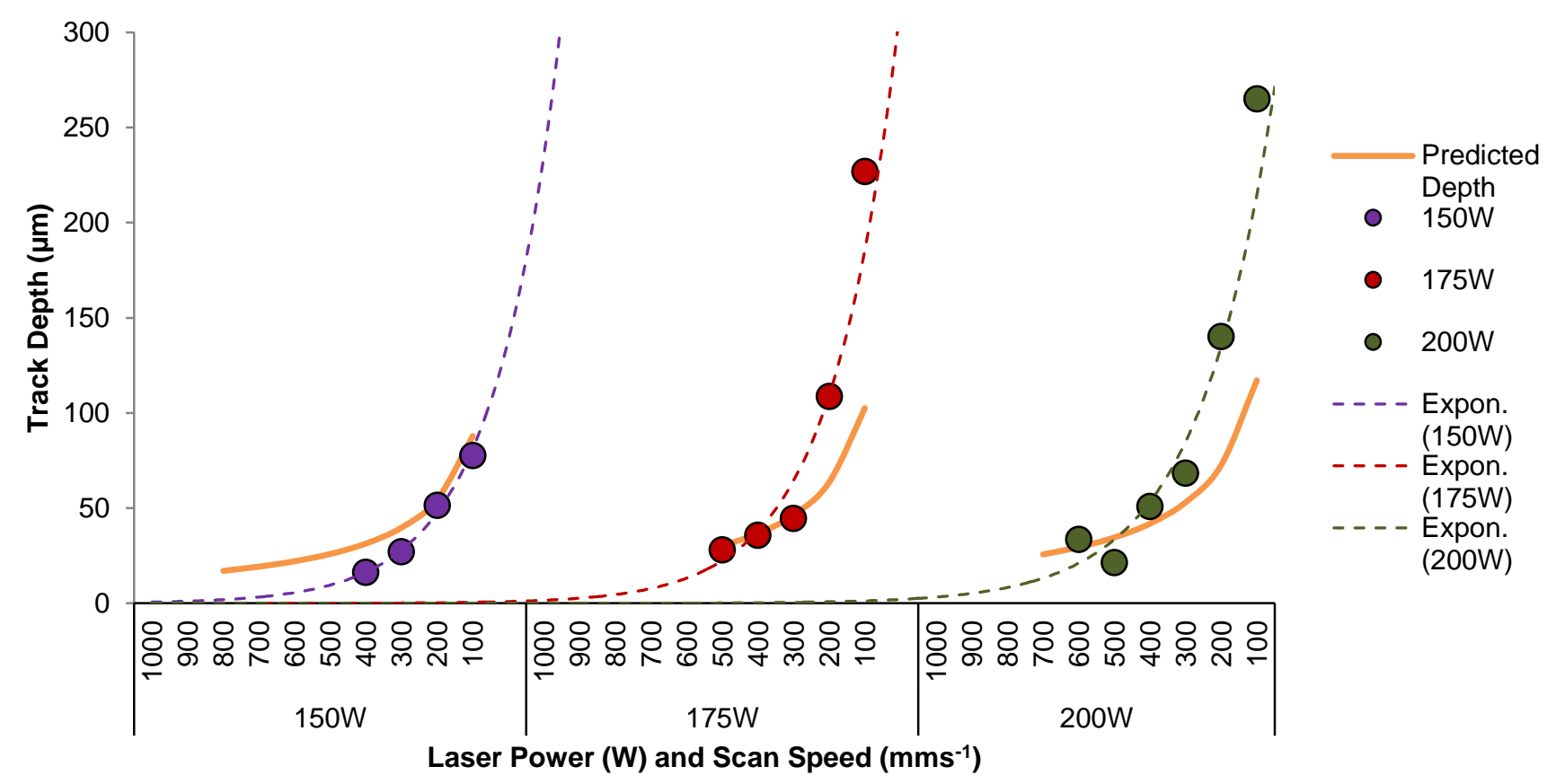

Figure 62. Experiment D (SS316L) - Comparison of measured and predicted penetration depths according to equation from Gladush and Smurov, [108].

\section{Depth-to-Width Contour Map}

Similar to the discussion section for experiment $B$, the line build percentage values and depth-to-width ratios for this experiment were used to build two contour maps, shown below in Figure 64 and Figure 65, respectively. A marker was used to indicate the laser power and scan speed used to create the optimal density from the DOE performed at the beginning of the chapter.

As can be expected, the line build percentages in Figure 64 are at their highest in regions of low scan speed and at high laser power. At most laser power settings, ranging between $100 \mathrm{~W}$ and $200 \mathrm{~W}$, scan speed can range between $100 \mathrm{mms}^{-1}$ and $300 \mathrm{mms}^{-1}$ to obtain $100 \%$ on line build percentage values. This range is limited to $100 \mathrm{mms}^{-}$at the lowest power settings of $75 \mathrm{~W}$, however a line build percentage of $90 \%$ could still be achieved at $200 \mathrm{mms}^{-1}$.

When compared to the same contour map made for experiment $B$, as seen in Figure 39 , the threshold for achieving $100 \%$ track completion at moderate scan speeds of $200 \mathrm{mms}^{-1}$ or $300 \mathrm{mms}^{-1}$ was achieved using lower values of laser power, a range of $125 \mathrm{~W}$ to $200 \mathrm{~W}$, as 
compared to a range of $150 \mathrm{~W}$ to $200 \mathrm{~W}$, during the crucible experiments. This is a similar observation to what was observed from the topographical maps, indicating that the threshold for achieving fully built tracks is lowered when building on substrates made using the laserpowder bed fusion process.

The parameters used for the optimal DOE would fall on the $98 \%$ line in the crucible contour plot. Tracks built in this region were continuous but displayed certain irregularities, as seen below in the left-most image of Figure 63. On the right of this image is a cross-section taken from the same track, displaying a track bead with a spherical shape with minimal penetration into the substrate. This cross-section may have been taken at a portion of the track where "beading up" of the melt may have occurred, as observed in the centre of the topographical image on the left.

In Figure 65, the depth-to-width ratios throughout the contour map remain relatively low, with the peak ratio of around 0.6 being achieved at the highest laser power and lowest scan speed combination in the top-right corner. From the cross-sectional analysis, tracks were observed with fairly deep penetrations into the track, however the depths achieved would not exceed the widths of the track bead.

In comparison to the depth-to-width contour map for Experiment B, seen in Figure 40, tracks built using the same parameters on the crucible substrates had much lower depth-to-width ratios for every parameter combination used for Experiment $B$. 

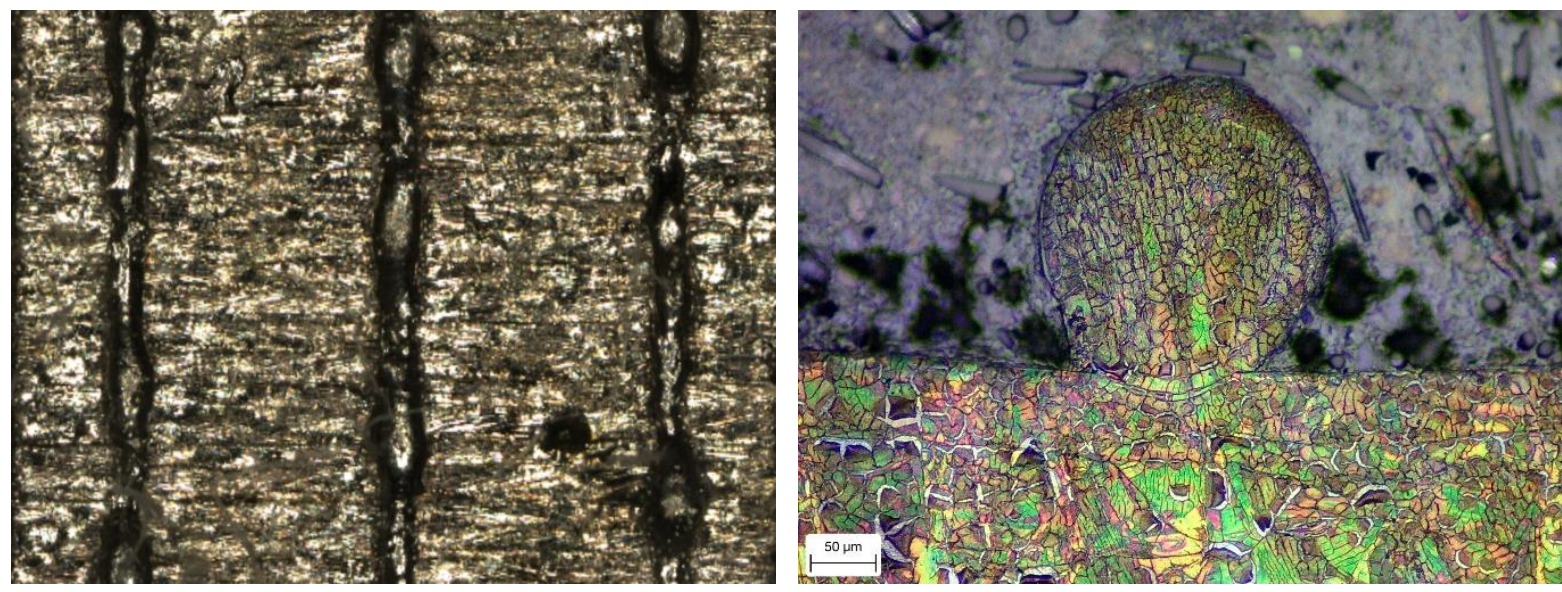

Figure 63. Experiment D (SS316L) - Left) Topographical image of track built using parameters similar to DOE optimal parameters, Right) cross-section taken at the same track.

(Track is 200W, $500 \mathrm{mms}^{-1}$, DOE is $190 \mathrm{~W}, 500 \mathrm{mms}^{-1}$ )

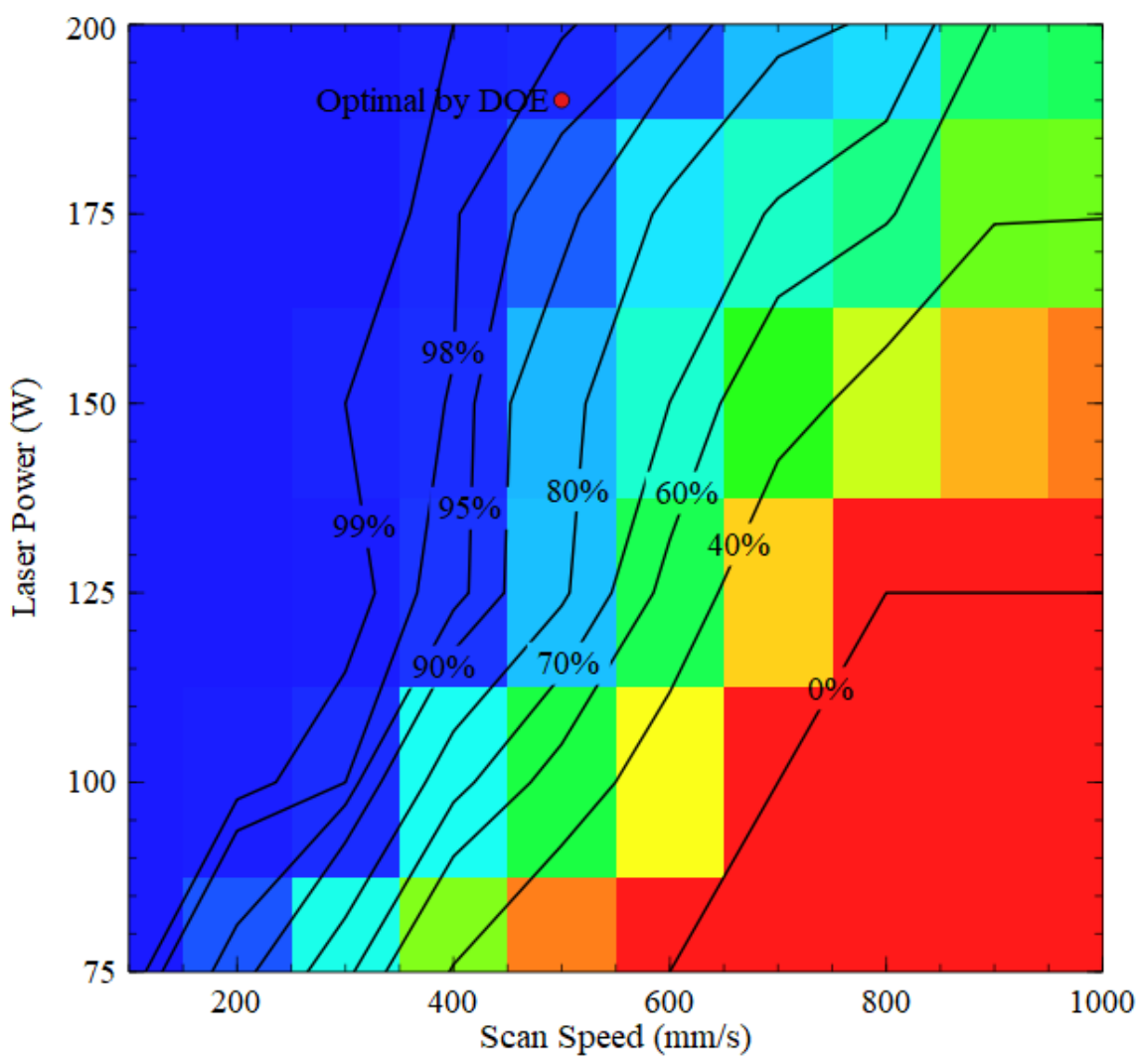

Figure 64. Experiment D (SS316L) - Contours of line build percentage of single tracks. 


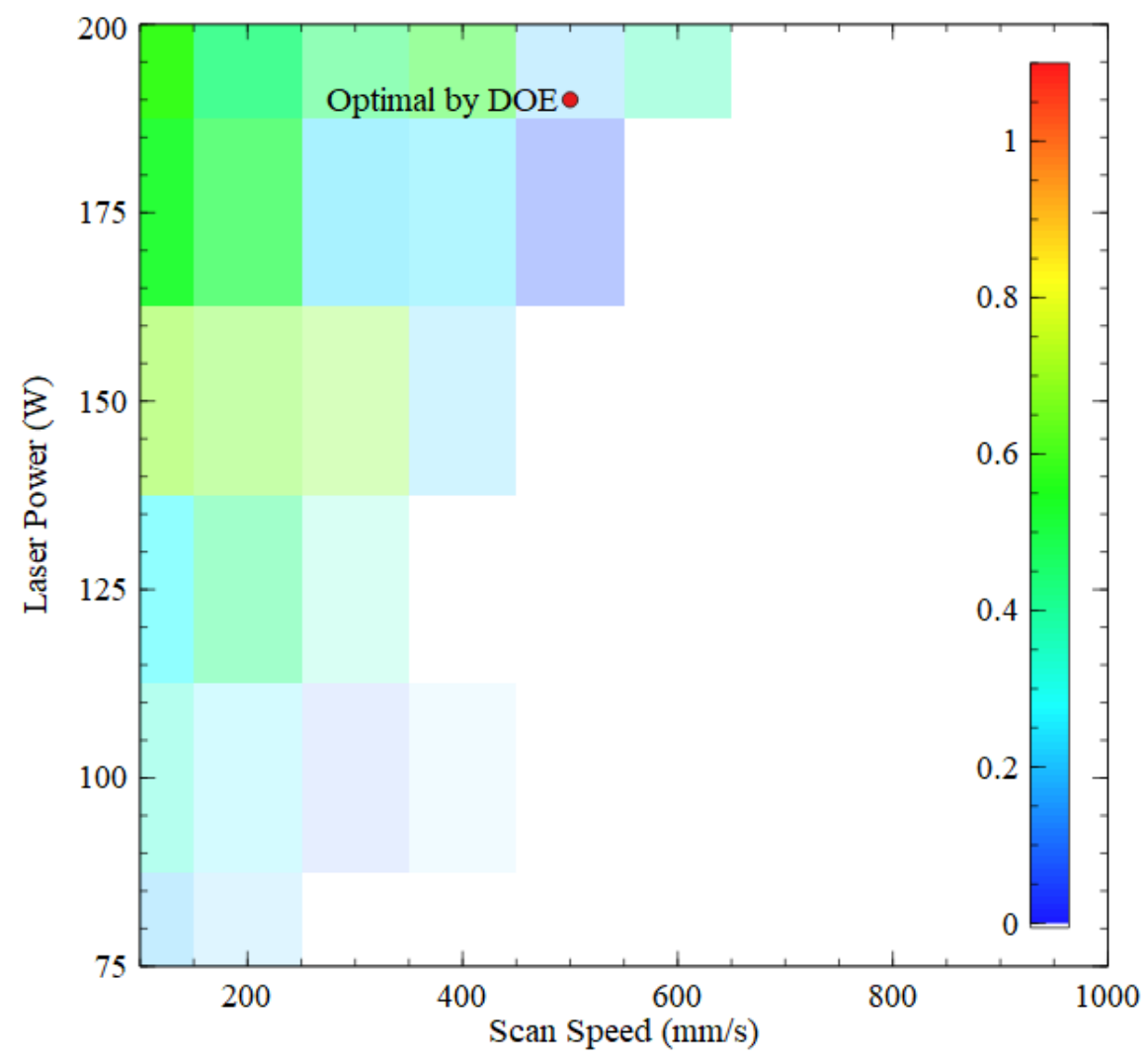

Figure 65. Experiment D (SS316L) - Contours of depth-to-width ratio.

\section{Microstructure of the Single Track}

Beraha II proved to be more effective at revealing the microstructure of stainless steel $316 \mathrm{~L}$ than the previously used Kalling's reagent. The substrate showed a semi-elliptical morphology, which overlap one another, representing the several multi-layered tracks that were used to make the bulk structure. For most of the single tracks, two distinct types of morphologies were observed. Columnar growth was observed at the lower ends of the bead, that is to say, towards the crucible and below the surface. At the edges of the bead which were not in contact with the substrate, smaller, cellular grains were observed. This observation is demonstrated in Figure 66 , which is a cross section of a track built at $175 \mathrm{~W}$ at $100 \mathrm{mms}^{-1}$. 


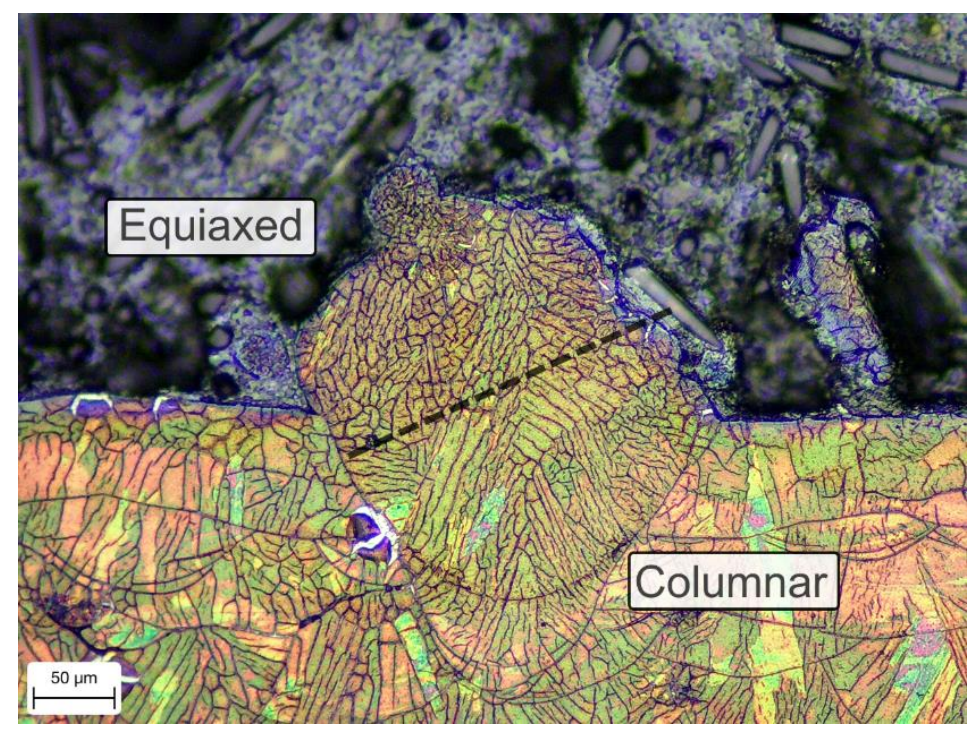

Figure 66. Experiment D (SS316L) - Cross section of a track, highlighting the type of grain formation.

The high energy input of the laser during laser-powder bed fusion occurs over a short period of time, leading to the superfast heating and melting the melt. The heating and cooling rate can be very high $\left(10^{3}-10^{8} \mathrm{~K} \mathrm{~s}^{-1}\right),[120],[121]$, at the boundaries of the small melt pool. This causes rapid solidification at the solid/liquid and gas/liquid interfaces, leading to the nucleation and formation of very fine, cellular grains. These can be seen at the very edges of the substrate/track boundary, and prominently on the upper exposed surface of the track. The thermal gradient decreases at the inner regions of the track, particularly at the lower end, resulting in columnar growth towards the direction of the last place to cool, [122], [123]. This region would be the centre of the melt pool, which has the highest temperature due to the previously discuss Gaussian distribution of the laser interaction. This is further evidenced by the observation that columnar growth was usually orientated towards the centre of the melt bead.

\subsubsection{Conclusions}

The single tracks manufactured from Experiment B from Chapter 4 were repeated in Experiment $\mathrm{D}$. The main difference was the use of a substrate made using the same material powder and within the same build process, referred to as the crucible substrate. In comparison, a mild steel insert was used for Experiment B. Otherwise, the same parameters 
to make the single-track formations were used, with approximately the same powder thickness.

It was found that track formation was significantly affected. Whilst the range at which smooth, continuous track could form had decreased, track build rate had increased throughout the process map. The former is attributed to the improved wettability of the melt on the rougher crucible surface. Tracks with significant fragmentation were found to build more successfully on the crucible, and would transition from highly fragmented tracks to mostly intact ones, such as at $100 \mathrm{~W}$ and $300 \mathrm{mms}^{-1}$, where the line build percentage increased from $73.9 \%$ to $98 \%$ between the two experiments. Regions within the process map where track failed to build on inserts were found to have increased track formation on crucibles, though this improvement would be limited to droplet formation. Keyhole mode melting was still observed at regions of high laser power and low scan speed, and the peak depth-to-width ratio was found to be 0.6 . However, the effect was less pronounced when compared to Experiment B, where the peak depth-to-width ratio was over 1.0. Keyhole pore formation was also reduced, with two only instances observed in Experiment D compared to the several instances in Experiment $B$.

The DOE optimal parameters used for Chapter 5 complimented the results obtained from this experiment. Single-tracks fabricated using near-similar process parameters produced continuous tracks with no sign of keyhole porosity, which is beneficial to optimising bulk density.

From the results in this experiment, within the range of the processing parameters used, the optimal combination of laser power and scan speed would be $200 \mathrm{~W}$ and $400 \mathrm{mms}^{-1}$ respectively. Tracks formed at these settings had been fabricated without evidence of keyhole mode melting and had a reasonably high line build percentage (99\%). 


\subsection{Experiment E-Crucible Single-Track experiments using Ti-}

\section{$6 A l-4 V$}

\subsubsection{Results}

For each layer thickness, two sets of process maps were produced. The first was assembled from the topographical images taken using the Alicona Infinite Focus microscope. The second was assembled from the images captured from the mounted cross sections taken from the crucible samples.

\section{0um Crucible Depth}

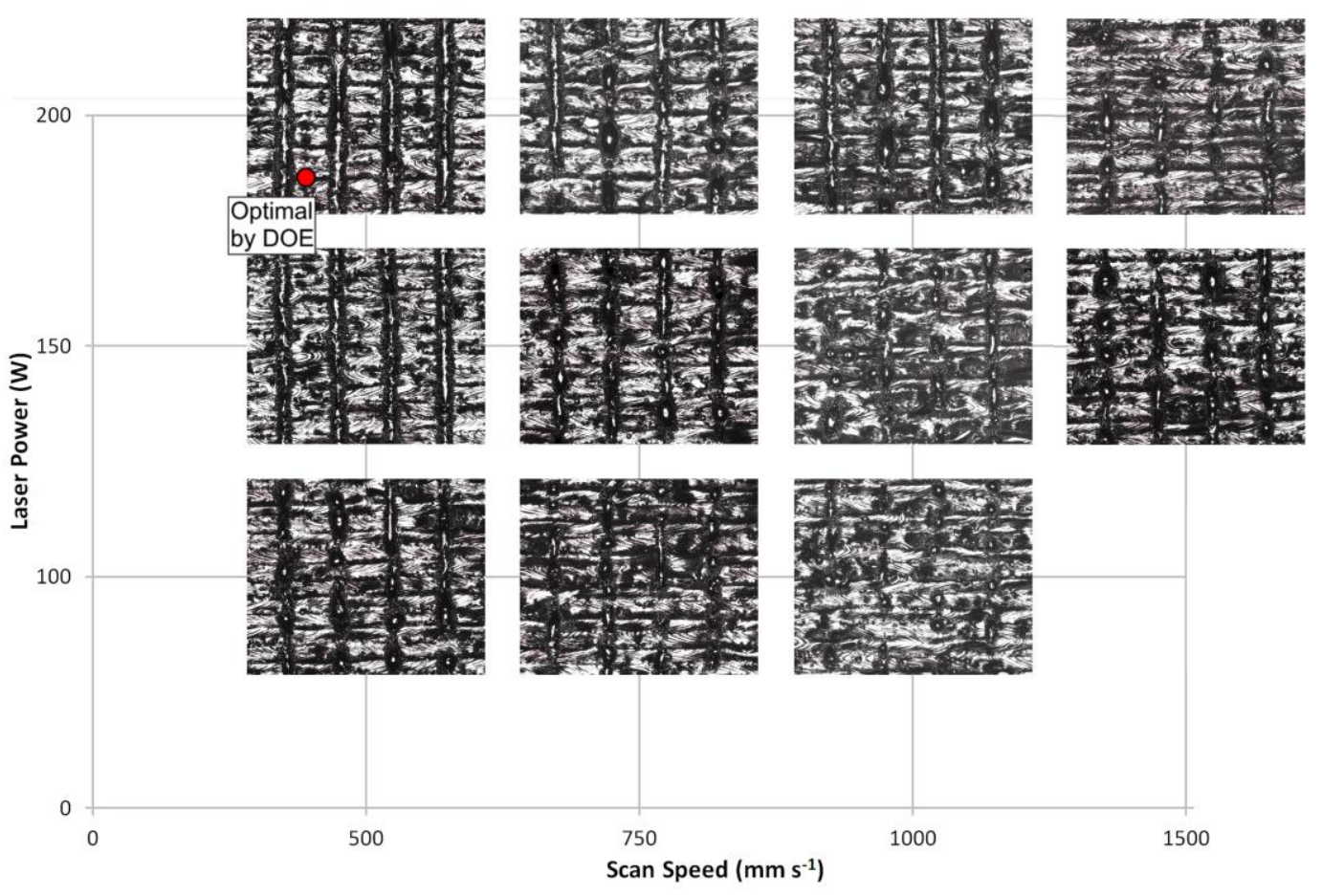

Figure 67. Experiment E (Ti-6Al-4V) - Topographical process map at 50 $\mu \mathrm{m}$ layer depth. The red dot shows the shows the parameters used at the Renishaw recommended operating conditions. 


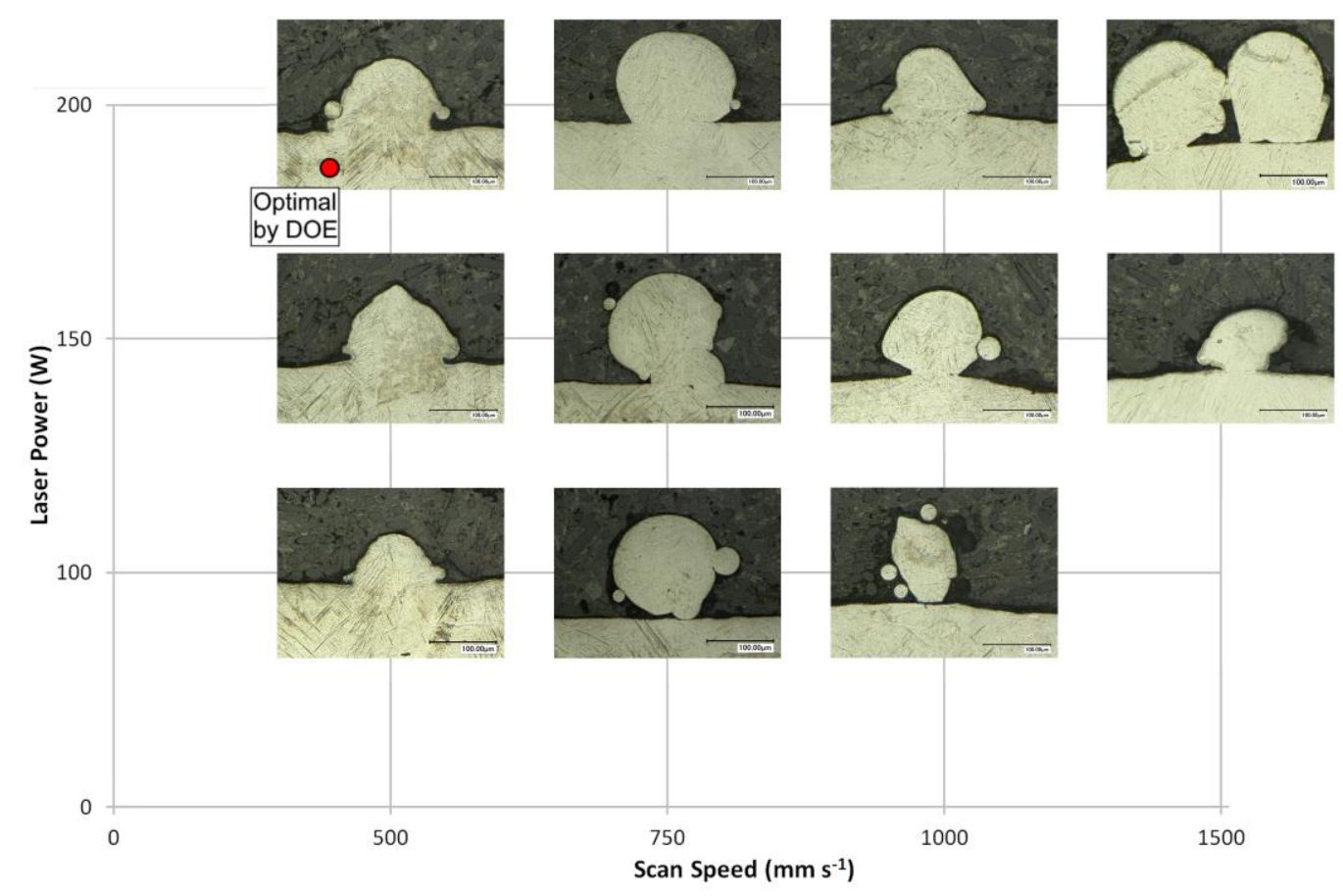

Figure 68. Experiment E (Ti-6Al-4V) - Cross-sectional process map at $50 \mu \mathrm{m}$ layer depth. The red dot shows the shows the parameters used at the Renishaw recommended operating conditions.

Tracks failed to form at speeds of $2000 \mathrm{mms}^{-1}$ and $3000 \mathrm{mms}^{-1}$, even at the highest laser power of 200W. Stable and continuous tracks formed at laser powers of $200 \mathrm{~W}$ and $150 \mathrm{~W}$ and scan speeds of $500 \mathrm{mms}^{-1}$. These parameters managed to build tracks successfully, at $99.9 \%$ and $86.3 \%$ line build percentages for $200 \mathrm{~W}$ and $150 \mathrm{~W}$, respectively. However, the tracks built had minor irregularities and did not take the smooth, even shapes of the tracks seen in Experiment A (Figure 25), which used 316L stainless steel powder. A direct comparison can be seen in Figure 69 below. The parameters obtained from the DOE at the beginning of the experiment, $185 \mathrm{~W}$ and $433 \mathrm{mms}^{-1}$ for a layer thickness of $50 \mu \mathrm{m}$, matched closely to the results obtained in this range, suggesting that single tracks of the DOE parameters would be fabricated in a similar manner. 

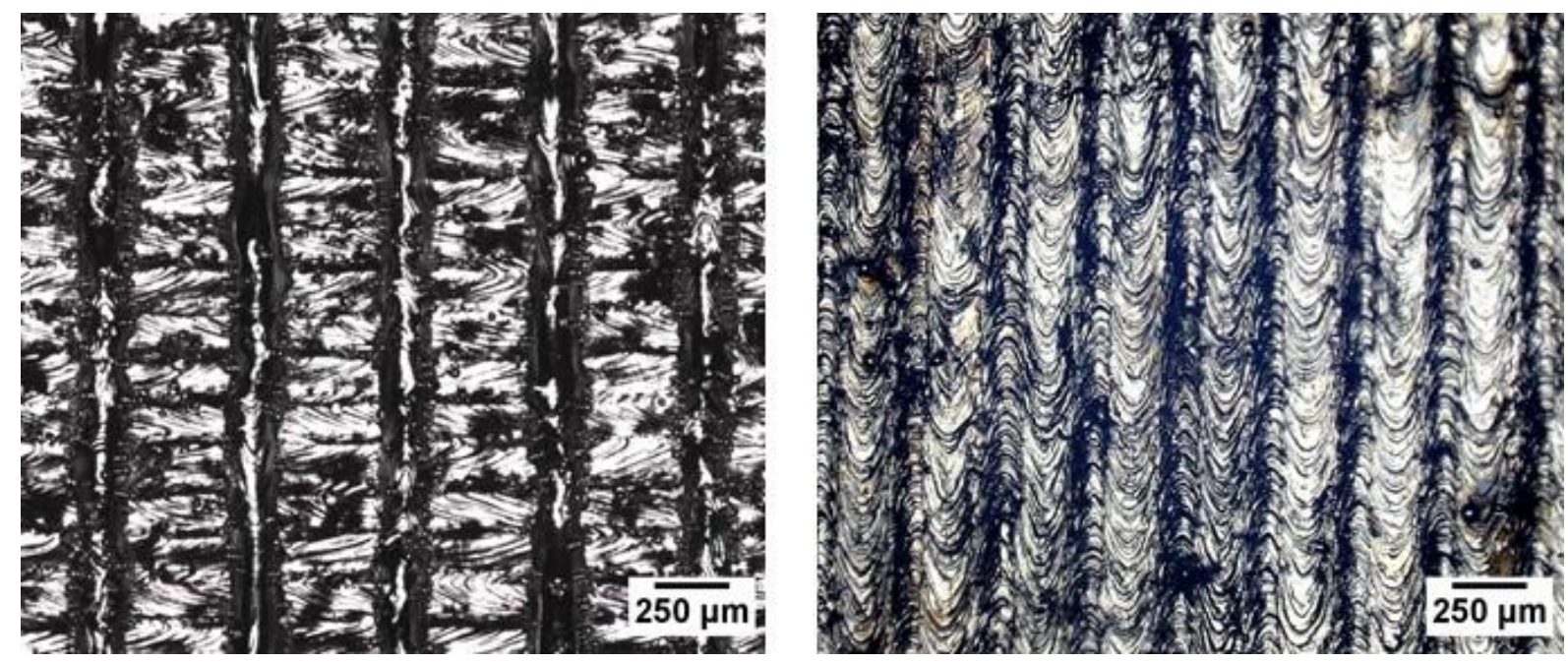

Figure 69. Left) Experiment E (Ti-6Al-4V) -Single Tracks, produced at 200W, $500 \mathrm{mms}^{-1}$.

Right) Experiment A (SS316L) - Single Tracks, produced at 200W, 433mms ${ }^{-1}$.

Cross sections taken at these settings showed spherical melt beads with good penetration into the crucible surface. At a laser power of $200 \mathrm{~W}$ and scan speed of $750 \mathrm{mms}^{-1}$, the tracks started to become more irregularly shaped and large droplets began to form. Kinks would appear in the track and variations in height would become more apparent. Gaps would form in some sections of the tracks. Large droplets would also begin to form, with peak heights of $200 \mu \mathrm{m}$ that were much larger than the average track height of $132 \mu \mathrm{m}$. The differences between the two types of lines formed at higher speeds are highlighted in Figure 70 below, with the dark blue/purple coloured regions signifying large droplet formations. 

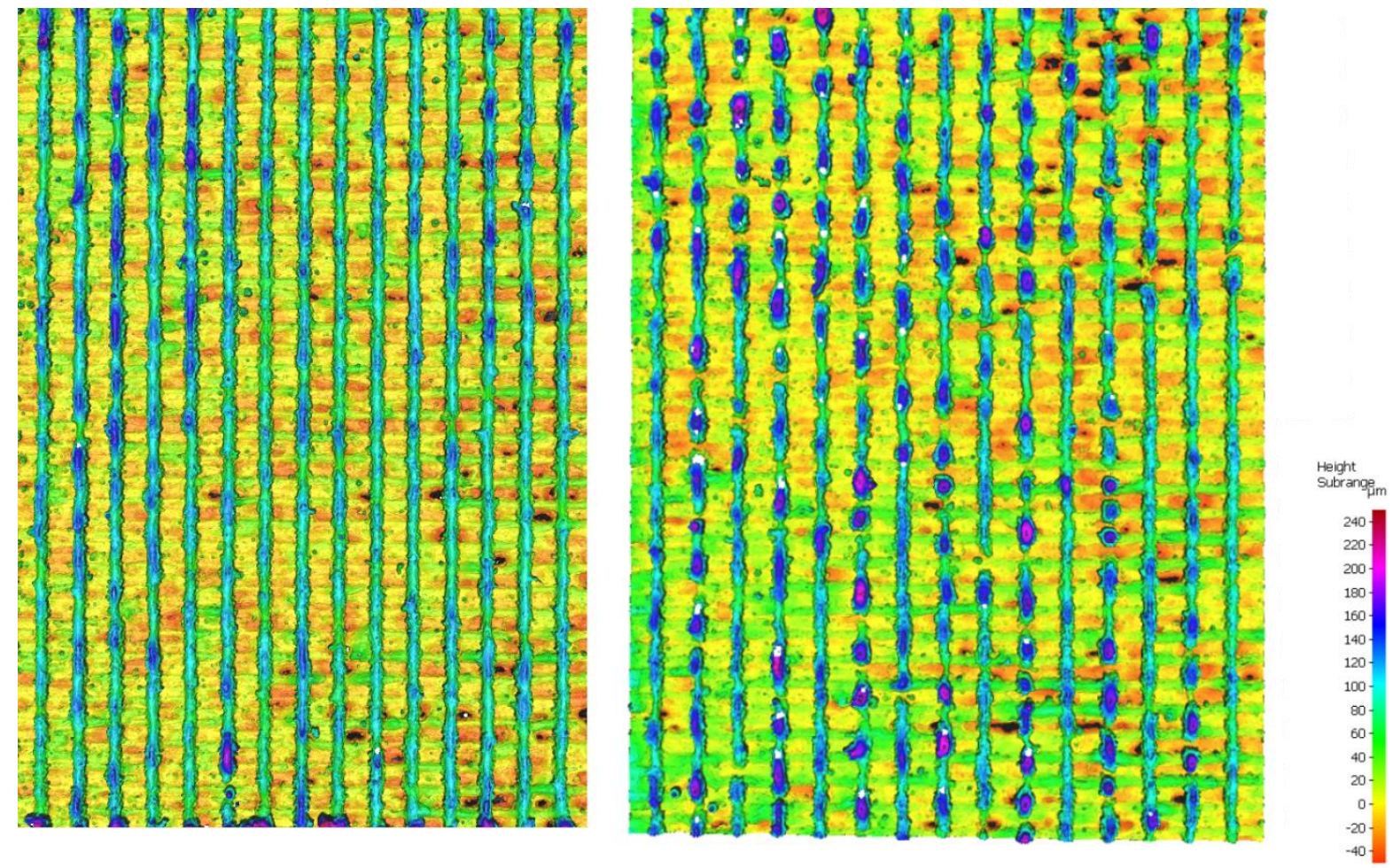

Figure 70. Experiment E (Ti-6Al-4V) - Left) Continuous tracks built at 200W, $500 \mathrm{mms}^{-1}$, Right) continuous tracks and droplet formation, built at $200 \mathrm{~W}, 750 \mathrm{mms}^{-1}$, crucible depth of $50 \mu \mathrm{m}$.

Tracks formed at $150 \mathrm{~W}$ laser power and with higher scan speeds of $750 \mathrm{mms}^{-1}$ and $1000 \mathrm{mms}^{-1}$ began to break up into droplets. These droplets would also reach peak heights much larger than that of continuous tracks, ranging between $200 \mu \mathrm{m}$ and $220 \mu \mathrm{m}$. Droplet formation would increase with the increase of scan speed.

In the cross sections taken from these tracks, the depth of laser penetration would be limited or not present at all. In such cases, the bead formed would appear to be completely detached and unsupported from the surface of the crucible. Most likely the bead is supported at a point out of the plane from where that particular track was sectioned. The presence of necking at the base of the bead would also be observed. It is described as a narrowing of the beads profile just above the surface of the substrate, and is clearly distinguished in Figure 71 below. 


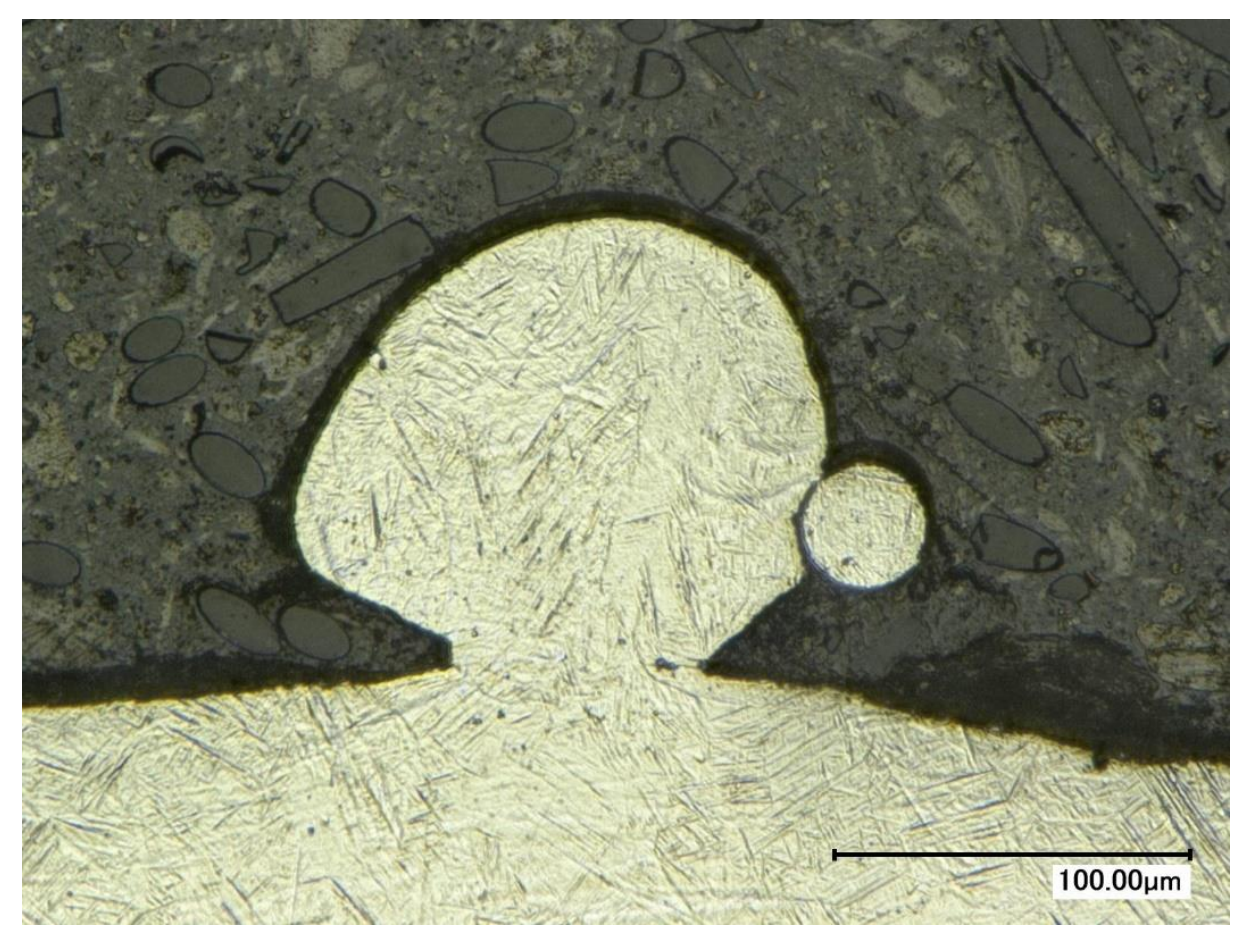

Figure 71.Experiment E (Ti-6Al-4V) - Track cross-section, taken at 150W, $1000 \mathrm{mms}^{-1}$. Necking occurs between the melt bead and substrate.

Tracks with similar geometries were observed at $200 \mathrm{~W}$ and higher scan speeds of $1000 \mathrm{mms}^{-1}$ and $1500 \mathrm{mms}^{-1}$. Track fragmentation would increase with scan speed, and the build percentage would decrease to $77.3 \%$ and $58.7 \%$ respectively.

At a laser power of $100 \mathrm{~W}$ and a scan speed of $500 \mathrm{mms}^{-1}$, tracks formed into a mix of small portions of continuous, irregular tracks and small droplets. Large sections of track would go unbuilt. Spherical melt beads would form with limited or no penetration into the underlying powder layer. At higher scan speeds of $500 \mathrm{mms}^{-1}$ and $750 \mathrm{mms}^{-1}$, droplet formation would increase. Cross sections taken at $750 \mathrm{mms}^{-1}$ showed irregularly shaped, non-rounded melt beads. 
100um Crucible Depth

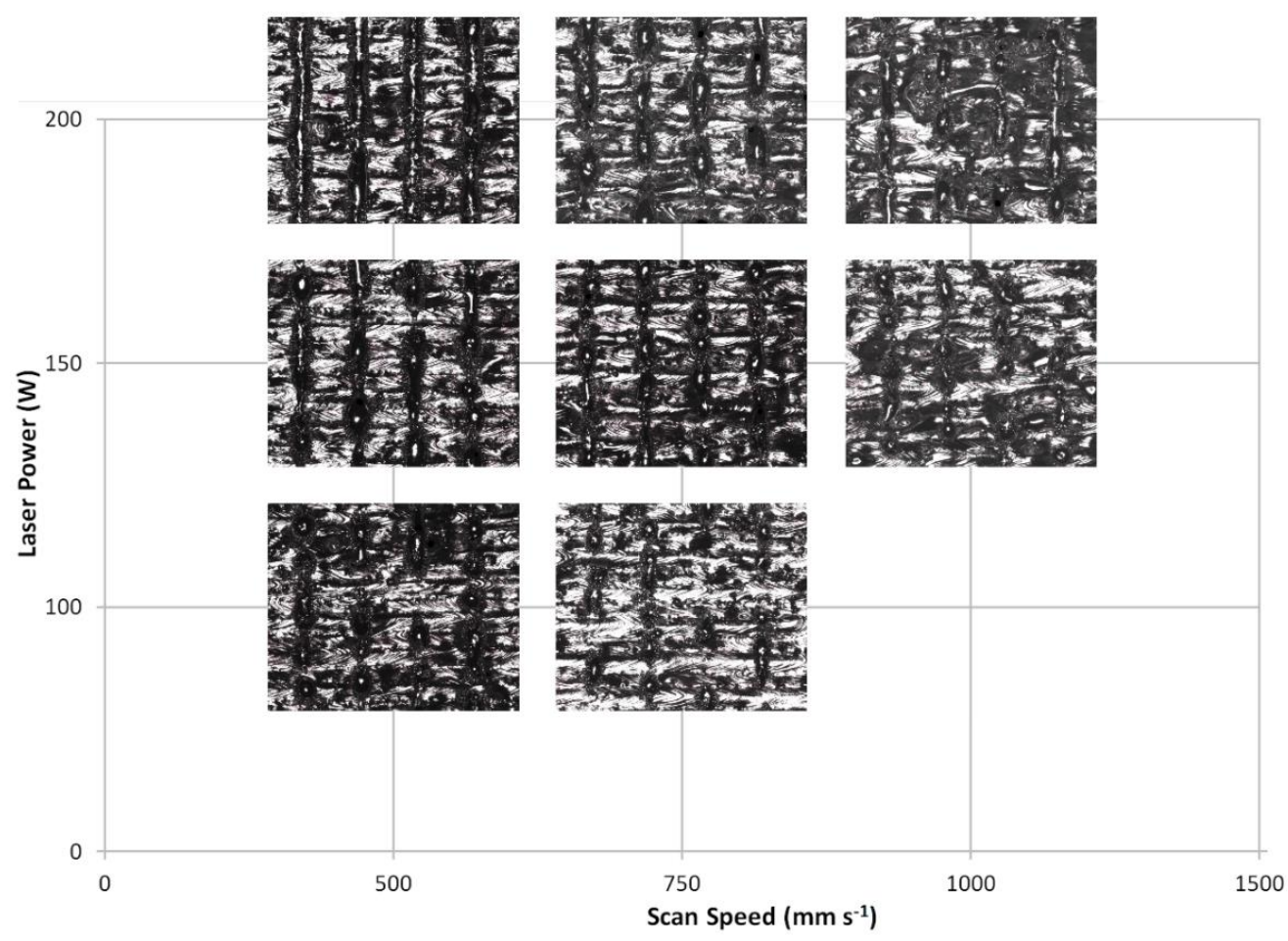

Figure 72. Experiment $\mathrm{E}$ (Ti-6Al-4V) - Topographical process map at $100 \mu \mathrm{m}$ layer depth.

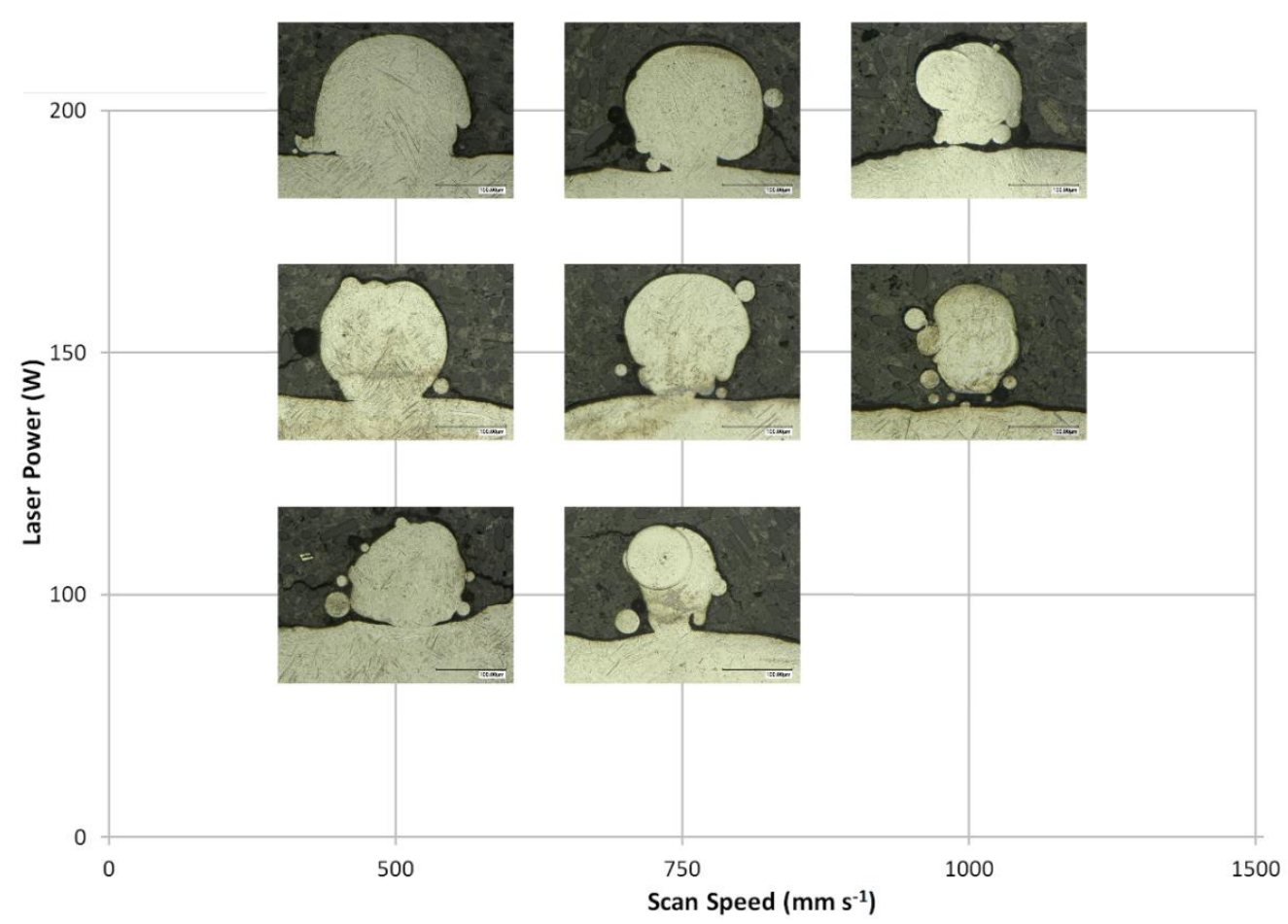

Figure 73. Experiment E (Ti-6Al-4V) - Cross-sectional process map at $100 \mu \mathrm{m}$ layer depth. 
Tracks failed to form at speeds ranging between $1500 \mathrm{mms}^{-1}$ and $3000 \mathrm{mms}^{-1}$ at this layer thickness. At a laser power of $200 \mathrm{~W}$ and a scan speed of $500 \mathrm{mms}^{-1}$, the tracks formed were mostly continuous, with $93.6 \%$ of the lines successfully being built. Cross sections of these tracks showed large circular melt beads with good penetration into the crucible substrate. However, colour mapping of the 3D data showed that the tracks built were highly irregular and uneven. Whilst the tracks remained largely unbroken, the tracks would solidify into large beads along the track, with height peaking at around $220 \mu \mathrm{m}$, well above the average of $129 \mu \mathrm{m}$. This can be observed in Figure 74.
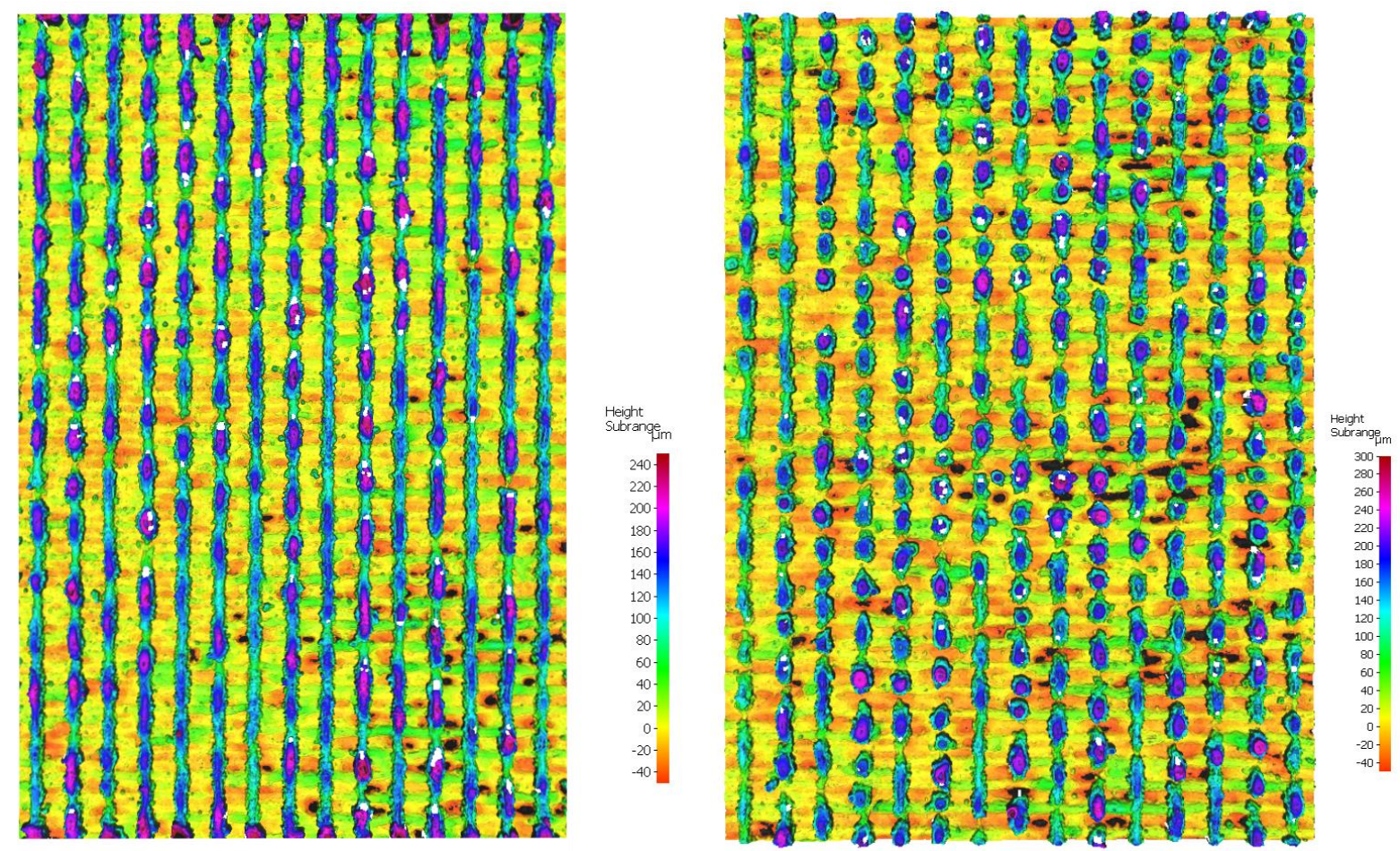

Figure 74. Experiment E (Ti-6Al-4V) - Left) Continuous tracks built at 200W, $500 \mathrm{mms}^{-1}$, Right) continuous tracks and droplet formation, built at $200 \mathrm{~W}, 750 \mathrm{mms}^{-1}$, crucible depth of $100 \mu \mathrm{m}$.

At higher scan speeds, the tracks would mostly form as large droplets, and the few sections of continuous tracks that did form were visibly distorted. Track build percentage would drop to $74.5 \%$ and $64.7 \%$ for $750 \mathrm{mms}^{-1}$ and $1000 \mathrm{mms}^{-1}$ respectively. The tracks formed at this speed would reach peak heights of around $260-280 \mu \mathrm{m}$, with the average heights of tracks formed being $170.0 \mu \mathrm{m}$ and $147.3 \mu \mathrm{m}$ respectively. The cross-sectional images showed that 
penetration into the substrate was limited, and that necking at the base of the bead was widespread.

At a laser power of $150 \mathrm{~W}$ and scan speed of $500 \mathrm{mms}^{-1}$, the tracks would mostly form as a series of large droplets. As scan speed increased, penetration into the crucible would decrease, with many cases of detachment from the previous layers being seen.

At a laser power of $100 \mathrm{~W}$, continuous track formation would very rarely be observed. Tracks would form into small droplets. The shape of the melt beads formed at $100 \mathrm{~W}$ laser power were all irregularly shaped, with the degree of this irregularity increasing with scan speed.
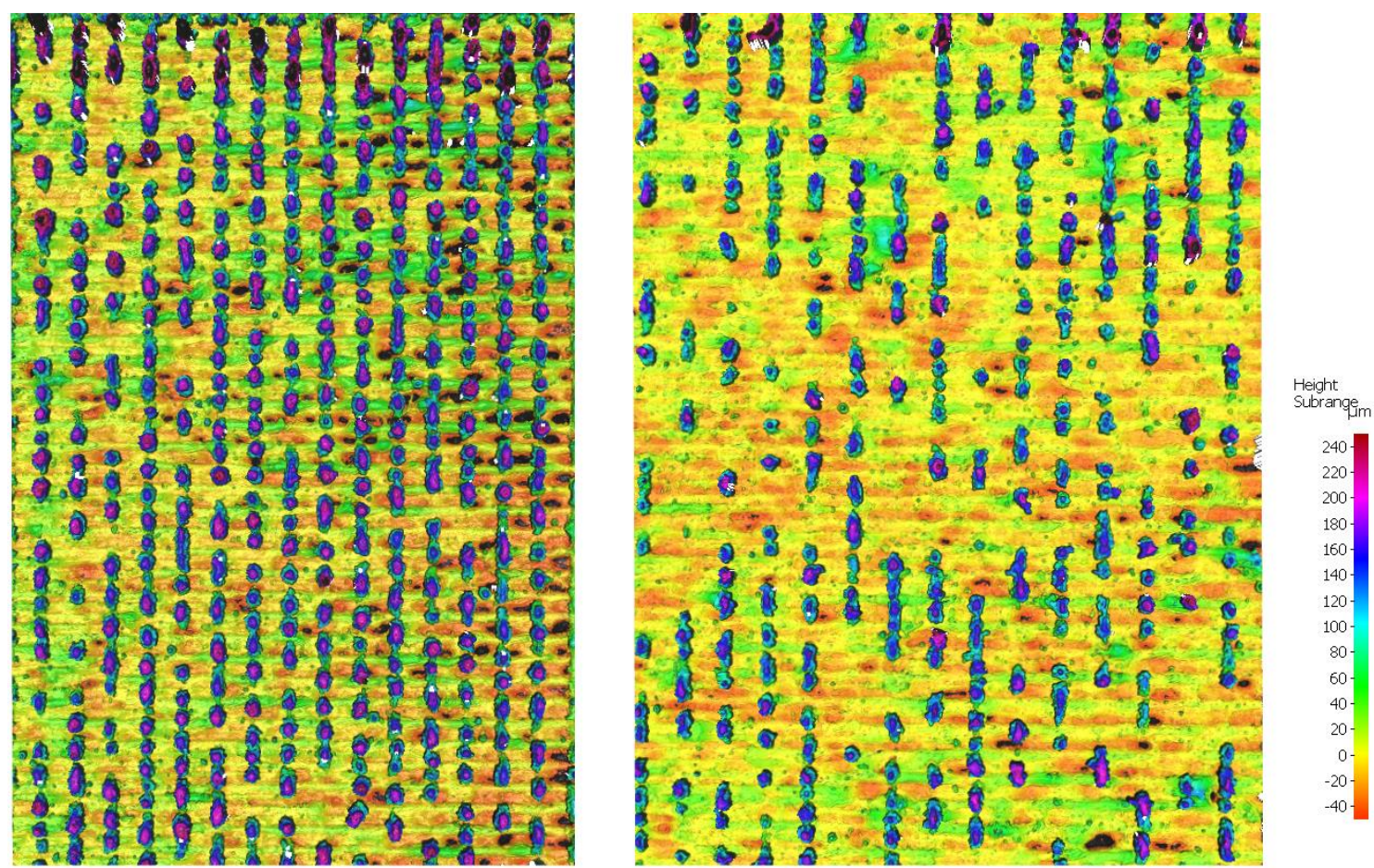

Figure 75. Experiment E (Ti-6Al-4V) - Left) Continuous tracks built at 100W, $500 \mathrm{mms}^{-1}$, Right) continuous tracks and droplet formation, built at $100 \mathrm{~W}, 750 \mathrm{mms}^{-1}$, crucible depth of $100 \mu \mathrm{m}$. 


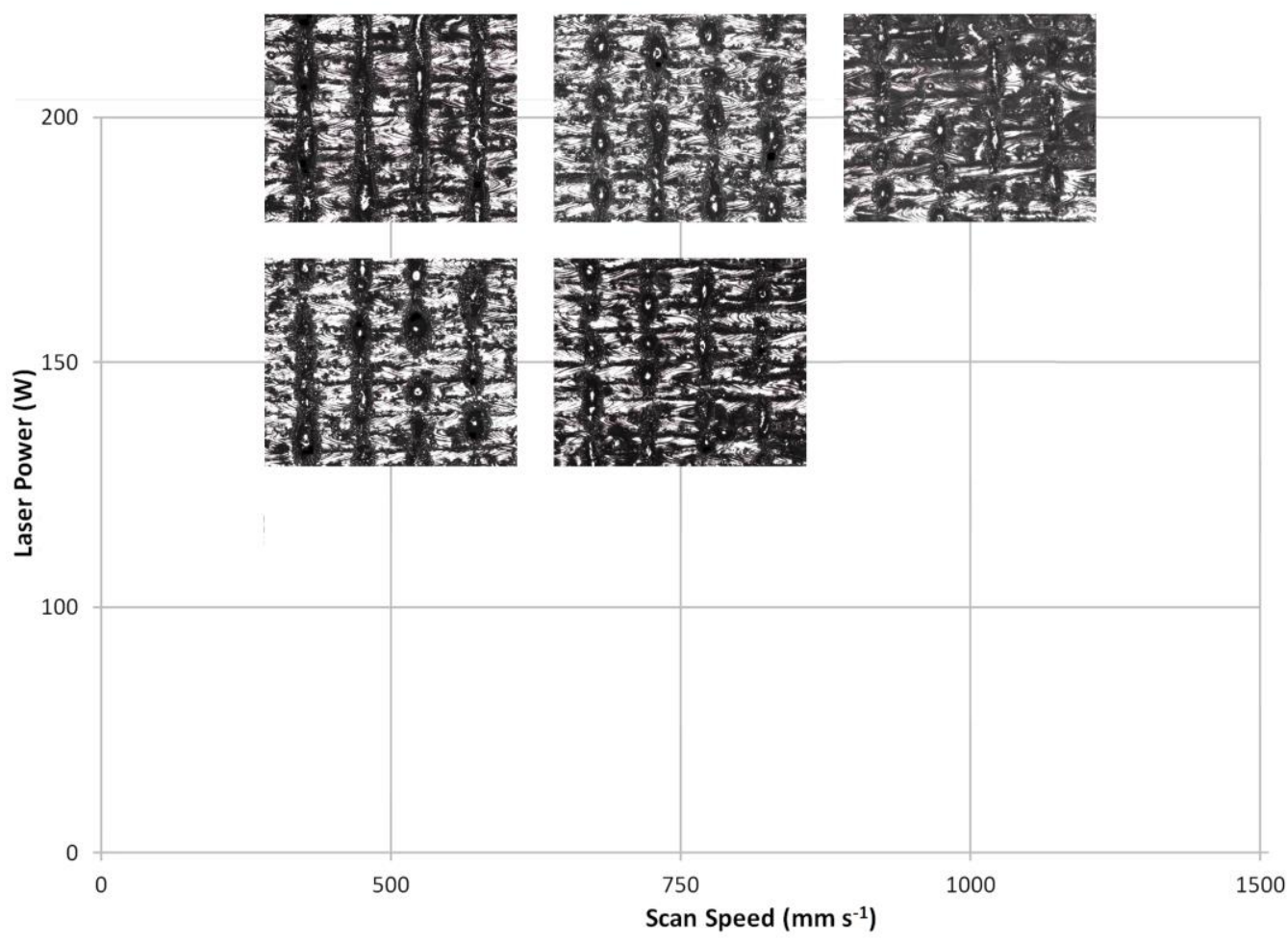

Figure 76. Experiment E (Ti-6Al-4V) - Topographical process map at $150 \mu \mathrm{m}$ layer depth.

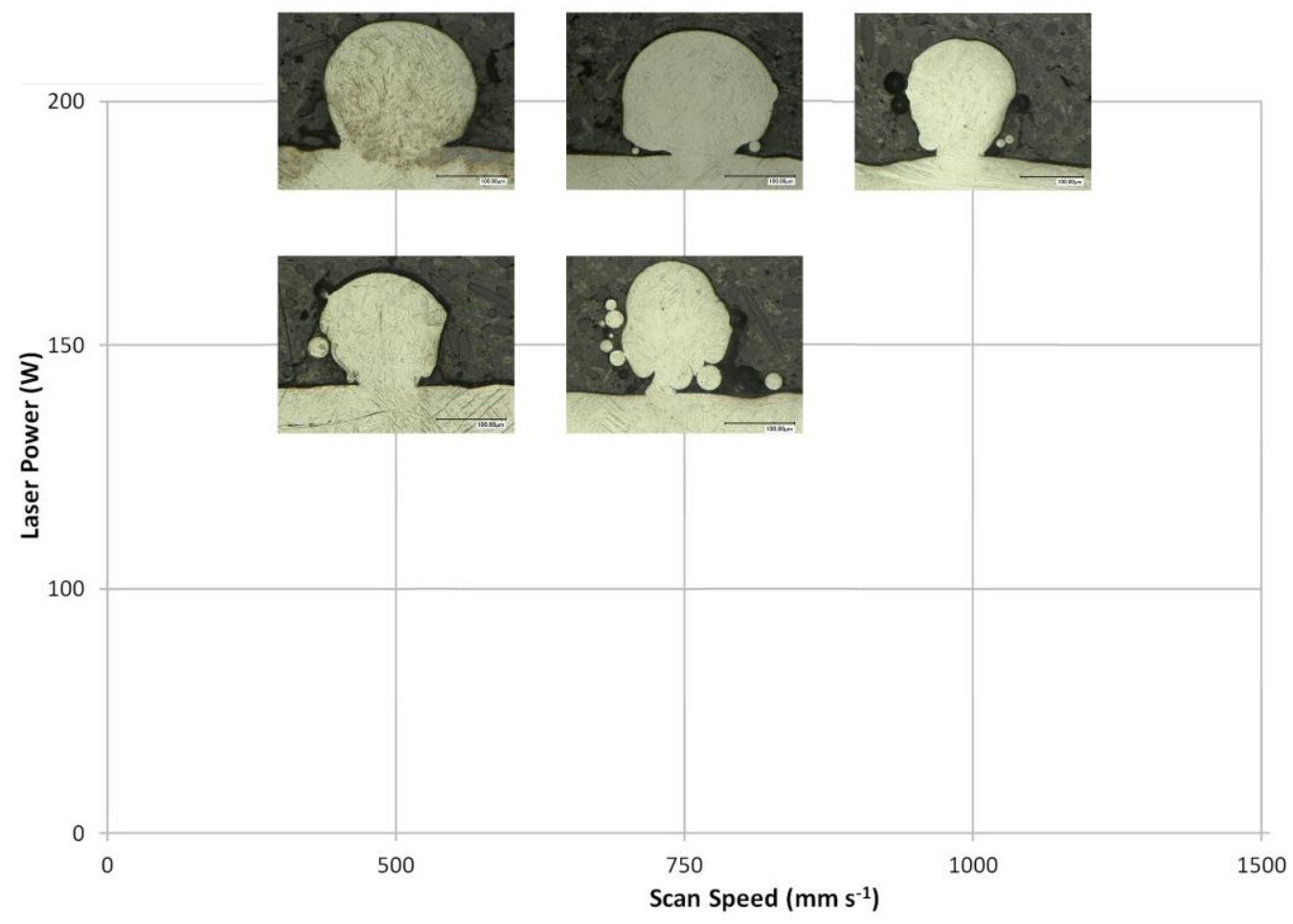

Figure 77. Experiment E (Ti-6Al-4V) - Cross-sectional process map at $150 \mu \mathrm{m}$ layer depth. 
Tracks had failed to form at scan speeds higher than $1000 \mathrm{mms}^{-1}$ at this layer thickness.

Tracks would fail to form with $100 \mathrm{~W}$ laser power.

At a laser power of $200 \mathrm{~W}$ and a scan speed of $500 \mathrm{mms}^{-1}$, tracks would build as continuous, irregular tracks and large droplets with peak height of around 220-280 $\mu \mathrm{m}$. Cross-sectional images of these tracks showed that penetration would range greatly. At higher scan speeds, large sections of the track would go unbuilt. Track build percentage would drop from $79.4 \%$ at $500 \mathrm{mms}^{-1}$ to $57.0 \%$ and $55.2 \%$ for $750 \mathrm{mms}^{-1}$ and $1000 \mathrm{mms}^{-1}$, respectively. Crosssectional images taken at higher scan speeds showed melt beads that remained mostly regular and spherically shaped. Necking and detachment from the crucible surface would become more prevalent at the highest scan speed of $1000 \mathrm{mms}^{-1}$.
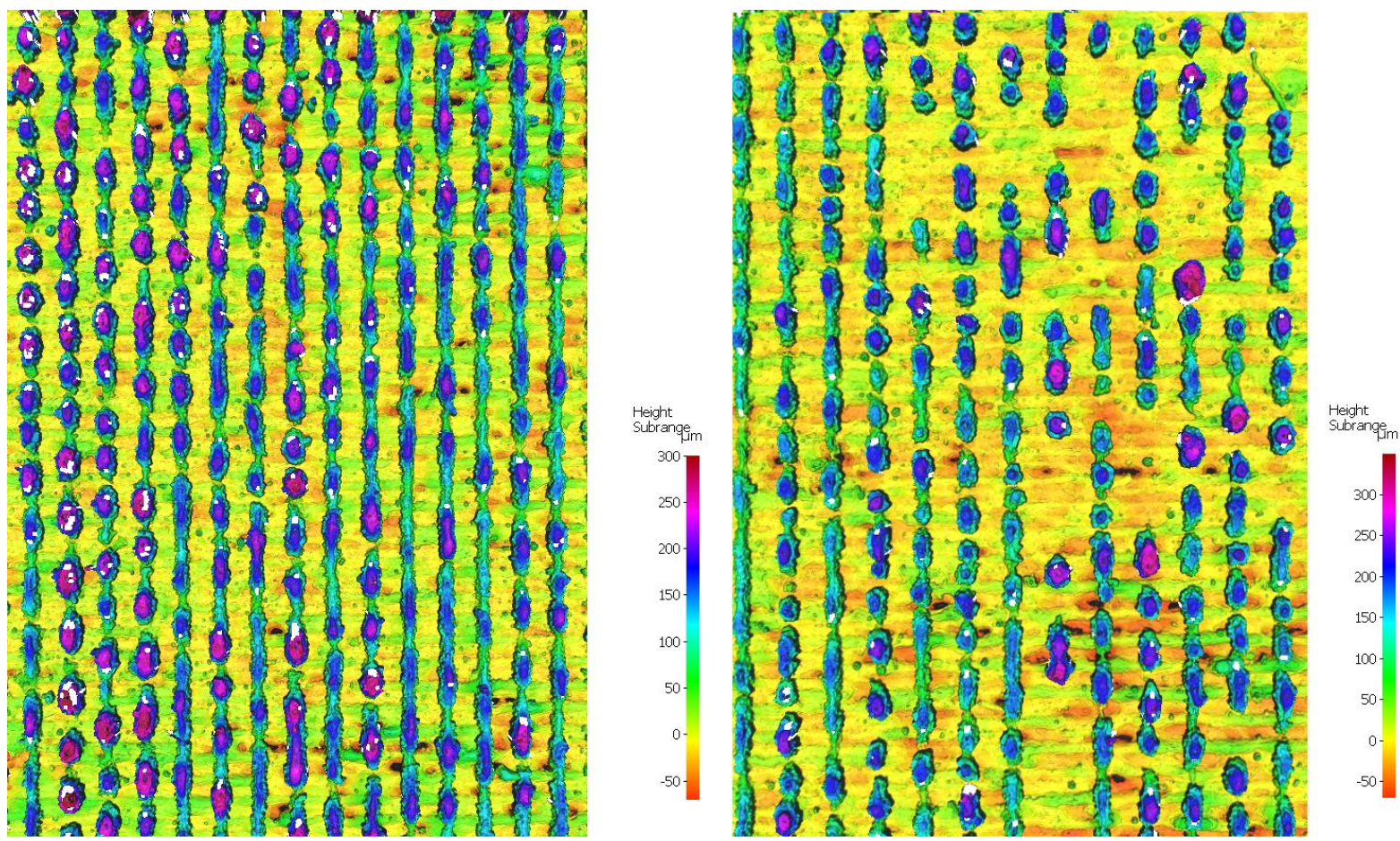

Figure 78. Experiment E (Ti-6Al-4V) - Left) Continuous tracks built at 200W, $500 \mathrm{mms}^{-1}$, Right) continuous tracks and droplet formation, built at $200 \mathrm{~W}, 750 \mathrm{mms}^{-1}$, crucible depth of $150 \mu \mathrm{m}$.

At a laser power of $150 \mathrm{~W}$, tracks would mostly form as a series of large droplets, with peak heights of around $260-280 \mu \mathrm{m}$. Large sections of the track would go unbuilt, with the average track build percentages being $62.3 \%$ and $59.0 \%$ for $500 \mathrm{mms}^{-1}$ and $750 \mathrm{mms}^{-1}$, respectively. A mix of large and small droplets would form at the higher scan speed of $750 \mathrm{mms}^{-1}$. Cross- 
sectional images revealed spherical track bead geometries with limited penetration and necking forming at $500 \mathrm{mms}^{-1}$. More irregularly shaped beads would form at $750 \mathrm{mms}^{-1}$, most often detached from the crucible.
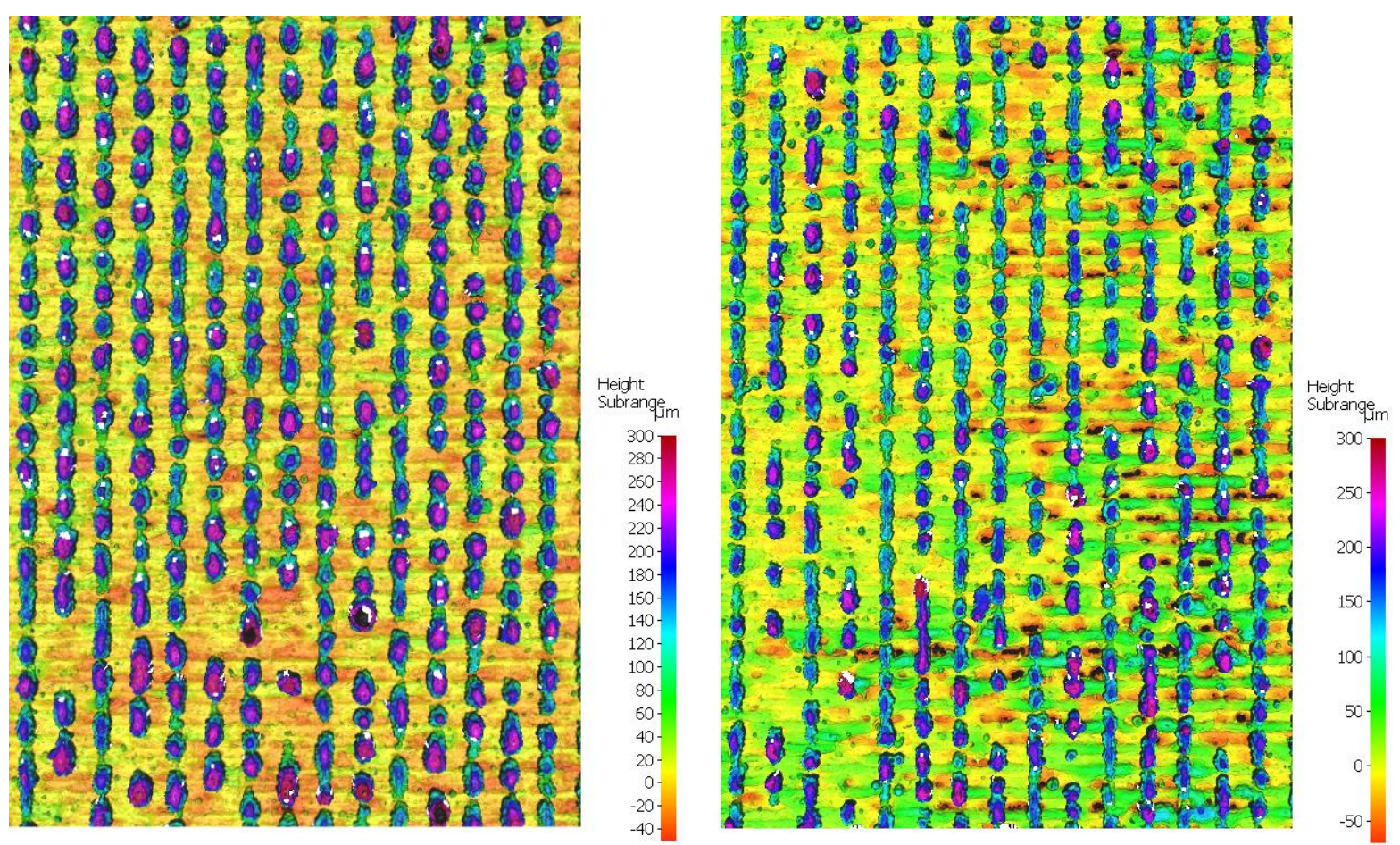

Figure 79. Experiment E (Ti-6Al-4V) - Left) Continuous tracks built at 150W, $500 \mathrm{mms}^{-1}$, Right) continuous tracks and droplet formation, built at $150 \mathrm{~W}, 750 \mathrm{mms}^{-1}$, crucible depth of $150 \mu \mathrm{m}$. 


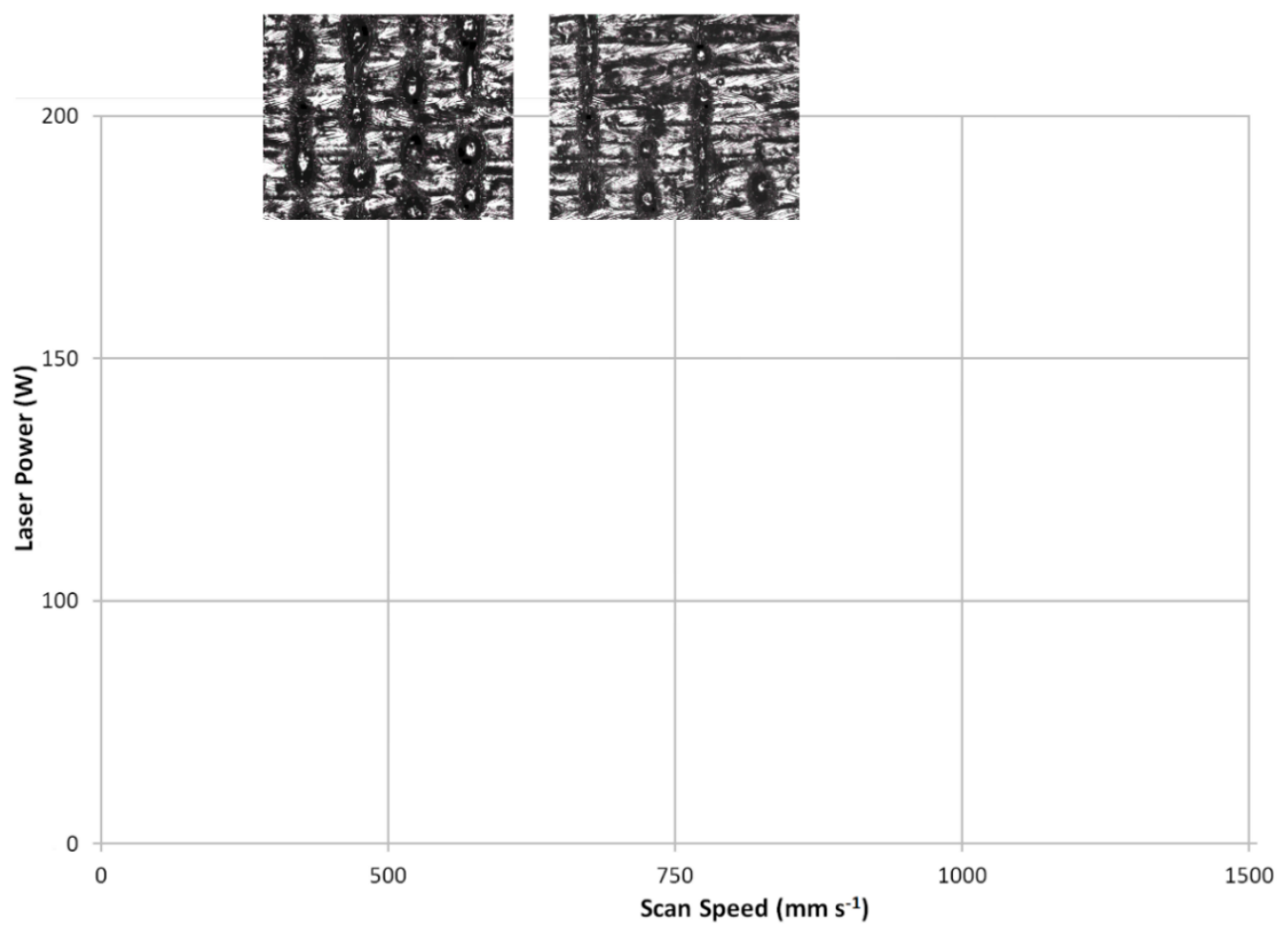

Figure 80. Experiment E (Ti-6Al-4V) - Topographical process map at $200 \mu \mathrm{m}$ layer depth.

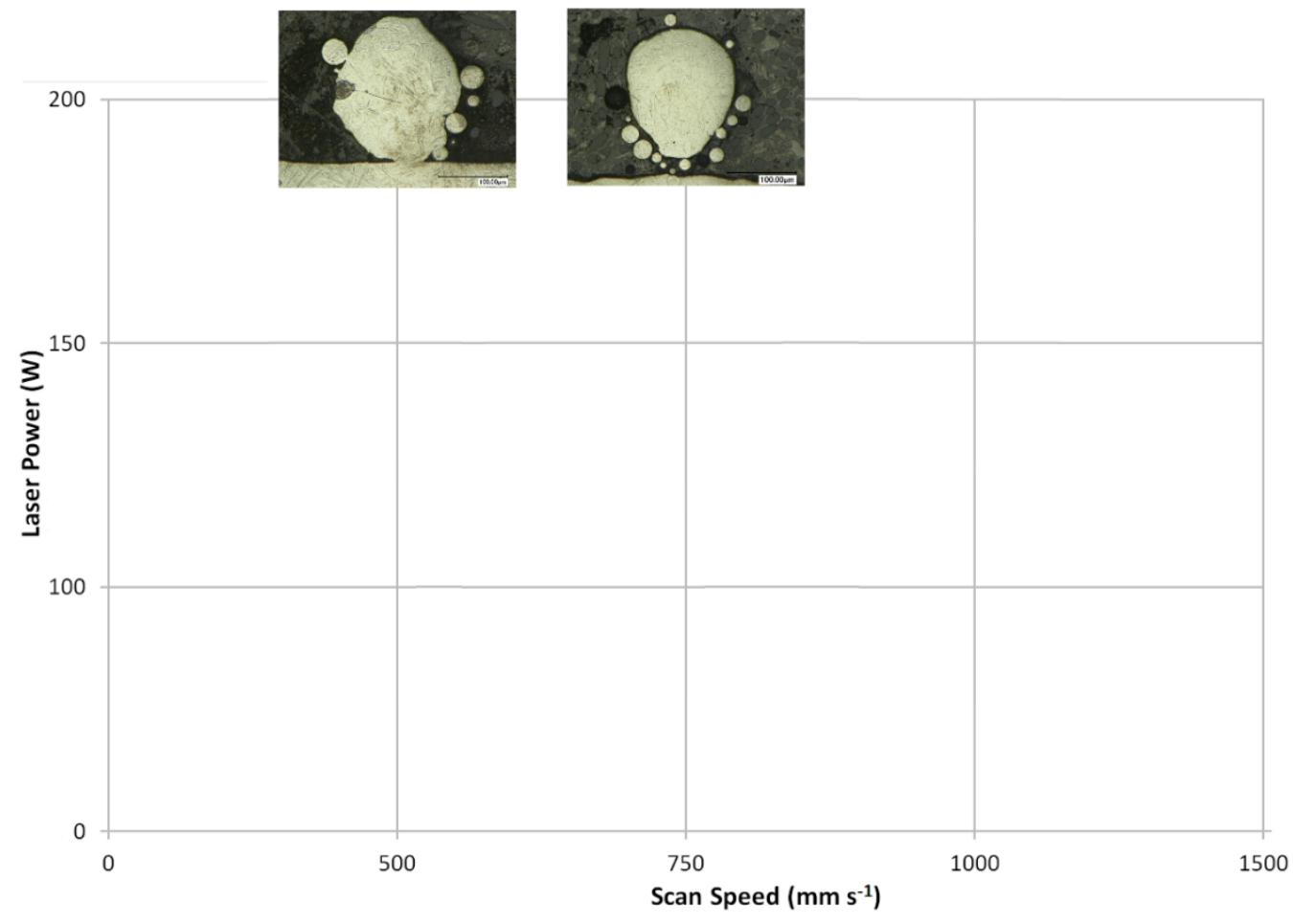

Figure 81. Experiment E (Ti-6Al-4V) - Cross-sectional process map at 200um layer depth. 
Tracks would only form at a laser power of $200 \mathrm{~W}$ at this layer thickness, at scan speeds of $500 \mathrm{mms}^{-1}$ and $750 \mathrm{mms}^{-1}$. The presence of satellite powder particles sintered to the melt bead was most frequently observed at this layer thickness.

At $500 \mathrm{mms}^{-1}$, large sections of track would fail to form, with only $57.3 \%$ of tracks being built. Tracks would form as large droplets, with peak heights of around $450 \mu \mathrm{m}$. Cross sections of the tracks showed melt beads with minimal or no penetration to the crucible. As scan speed increased, larger sections of the track would fail to build, decreasing to $33.9 \%$ for $750 \mathrm{mms}^{-1}$. However, droplet formation would decrease, and short segments of irregular, continuous tracks would appear. The peak height of droplets would also lower, reaching only 300 $400 \mu \mathrm{m}$. There was also a noted drop in the average height, from $317.6 \mu \mathrm{m}$ at $500 \mathrm{mms}^{-1}$, to $218.7 \mu \mathrm{m}$ in $750 \mathrm{mms}^{-1}$. The melt bead would become more irregularly shaped with increasing scan speed, with instances of extremely long necking, reaching half of the height of the bead, becoming more prevalent.
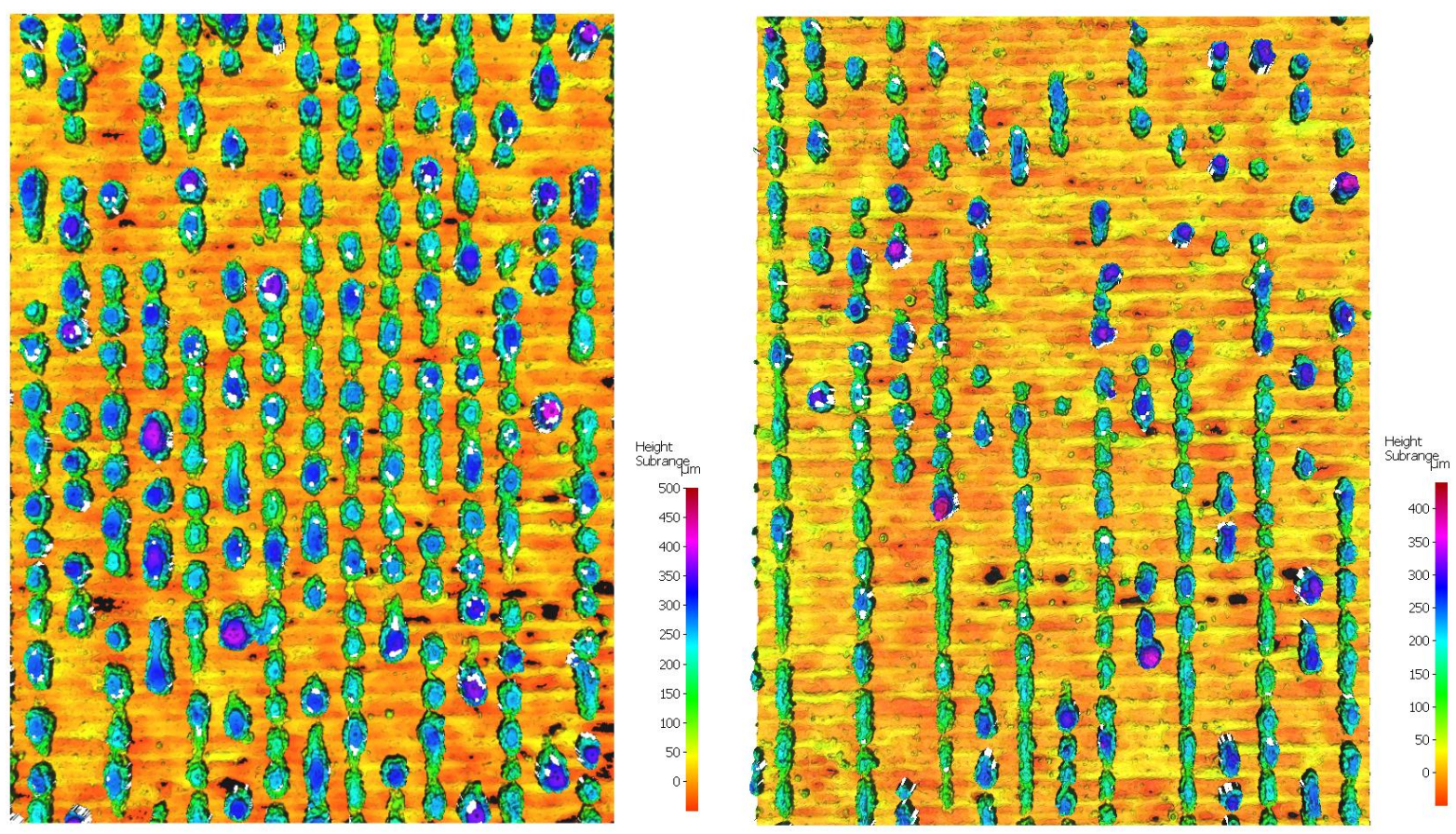

Figure 82. Experiment E (Ti-6Al-4V) - Left) Continuous tracks built at 200W, $500 \mathrm{mms}^{-1}$, Right) continuous tracks and droplet formation, built at $200 \mathrm{~W}, 750 \mathrm{mms}^{-1}$, crucible depth of $200 \mu \mathrm{m}$. 


\begin{tabular}{|c|c|c|c|c|c|c|c|}
\hline & \multicolumn{7}{|c|}{$50 \mu \mathrm{m}$} \\
\hline \multirow{3}{*}{ 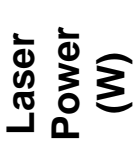 } & 200 & 99.9 & 77.2 & 63.3 & 58.7 & 0 & 0 \\
\hline & 175 & 86.3 & 75.7 & 74.7 & 50 & 0 & 0 \\
\hline & 150 & 64.7 & 66.4 & 49.3 & 0 & 0 & 0 \\
\hline
\end{tabular}

\begin{tabular}{|c|c|c|c|c|c|c|c|}
\hline & \multicolumn{7}{|c|}{$100 \mu \mathrm{m}$} \\
\hline \multirow{3}{*}{ 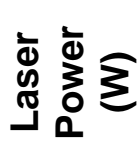 } & 200 & 93.6 & 74.5 & 64.7 & 0 & 0 & 0 \\
\hline & 175 & 74.8 & 65.3 & 61.1 & 0 & 0 & 0 \\
\hline & 150 & 64.7 & 38.1 & 0 & 0 & 0 & 0 \\
\hline
\end{tabular}

\begin{tabular}{|c|c|c|c|c|c|c|c|}
\hline & \multicolumn{7}{|c|}{$150 \mu \mathrm{m}$} \\
\hline \multirow{3}{*}{ 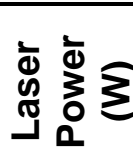 } & 200 & 79.4 & 57 & 55.2 & 0 & 0 & 0 \\
\hline & 175 & 62.3 & 59 & 0 & 0 & 0 & 0 \\
\hline & 150 & 0 & 0 & 0 & 0 & 0 & 0 \\
\hline
\end{tabular}

\begin{tabular}{|c|c|c|c|c|c|c|c|}
\hline \multirow{4}{*}{ 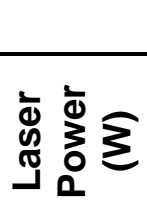 } & \multicolumn{7}{|c|}{$200 \mu \mathrm{m}$} \\
\hline & 200 & 57.3 & 33.9 & 0 & 0 & 0 & 0 \\
\hline & 175 & 0 & 0 & 0 & 0 & 0 & 0 \\
\hline & 150 & 0 & 0 & 0 & 0 & 0 & 0 \\
\hline & & 500 & 750 & 1000 & 1500 & 2000 & 3000 \\
\hline & & \multicolumn{6}{|c|}{ Scan Speed $\left(\mathrm{mms}^{-1}\right)$} \\
\hline
\end{tabular}

Table 17. Experiment E (Ti-6Al-4V) - Process map with line build percentages for each layer depth, given in bold over each table.

\subsubsection{Discussion}

The causes of track instability due to low laser power and high scan speed have been discussed in the previous chapters extensively. In this section, track instability, melt wettability and heat transfer into the substrate are discussed as a factor of layer thickness will be discussed.

At the top-most row of in Figure 68 , at a laser power of $200 \mathrm{~W}$, and with scan speed ranging between $500 \mathrm{mms}^{-1}$ and $1000 \mathrm{mms}^{-1}$, the track beads formed clearly show penetration into the previous substrate layer. This implies that the energy supplied by the laser was sufficient enough to heat the melt enough to cause wetting and spreading over the previous layer, causing melting of both the powder bed and the previous layer. With a larger layer thickness, a larger volume of powder would need to be melted in order for the resulting melt pool to 
come into contact with the substrate. Additionally, the melt bead that does form has much greater contact with surrounding powder material due to increased distance from the substrate and reduced wettability and fluid flow, as discussed in Experiment $\mathrm{C}$. If these particles bind to the molten metal but fail to reach melting temperatures, they remain as solids and the resulting mixture increases the viscosity of the melt pool due to the solids' effect on impeding fluid flow [71], [124].

These factors impede the ability for the melt pool to achieve a suitable wetting angle to the previous layer, and the bulk of the melt bead forms above the substrate. Using a high laser power, low scan speed, low layer thickness or any suitable combination of these process parameters may result in increased penetration into the substrate, as partly observed the second image in Figure 83. This could increase the temperature of the melt, thereby increasing the melt volume and improve the contact between the melt and the substrate, preventing instability in the track and allow for the formation of continuous tracks, made with a layer thickness larger than $50 \mu \mathrm{m}$. However, for all the parameter combinations used in this experiment, all failed to form continuous tracks, that is to say, $100 \%$ or near $-100 \%$ line build percentage, at layer thicknesses larger than $50 \mu \mathrm{m}$. Increasing the layer thickness resulted in a decrease in track stability and an increase in the balling phenomenon, as seen in Figure 83 below.
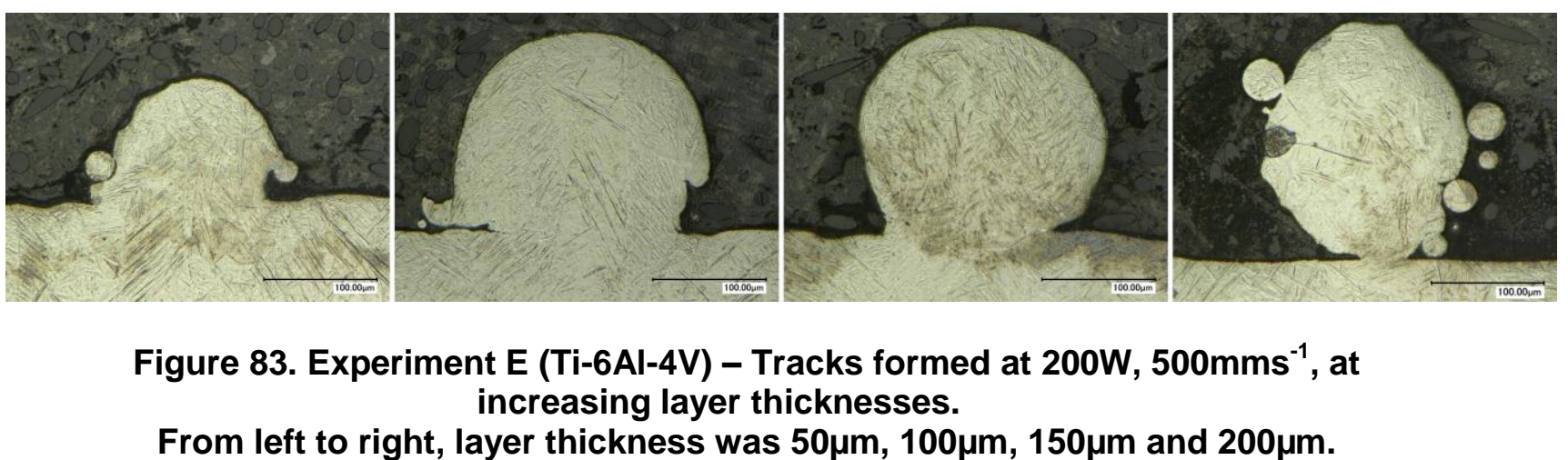

Additionally, powder particles sintered to the track were more commonly observed in crosssections taken at high layer thickness, such as the right-most cross-section in Figure 83, which was taken from a track made from a $200 \mu \mathrm{m}$ layer thickness. 
The average widths were recorded by measuring the widest possible diameter from the cross section of the track. The results were plot against scan speed for each laser power setting used, as seen in Figure 84, Figure 85 and Figure 86. The different lines on each plot show the average width values at each layer thickness.

At the 200W plot in Figure 84, the lowest track widths were observed at the $50 \mu \mathrm{m}$ layer thickness, and did not tend to vary greatly with increasing scan speed. As layer thickness increased, the width of the observed structures increased slightly as well. This trend was also noted at lower laser power settings, as seen in Figure 85 and Figure 86 . The increase in average track width at increasing layer thicknesses could be attributed to the increased balling formations.

Average Track Widths - 200W

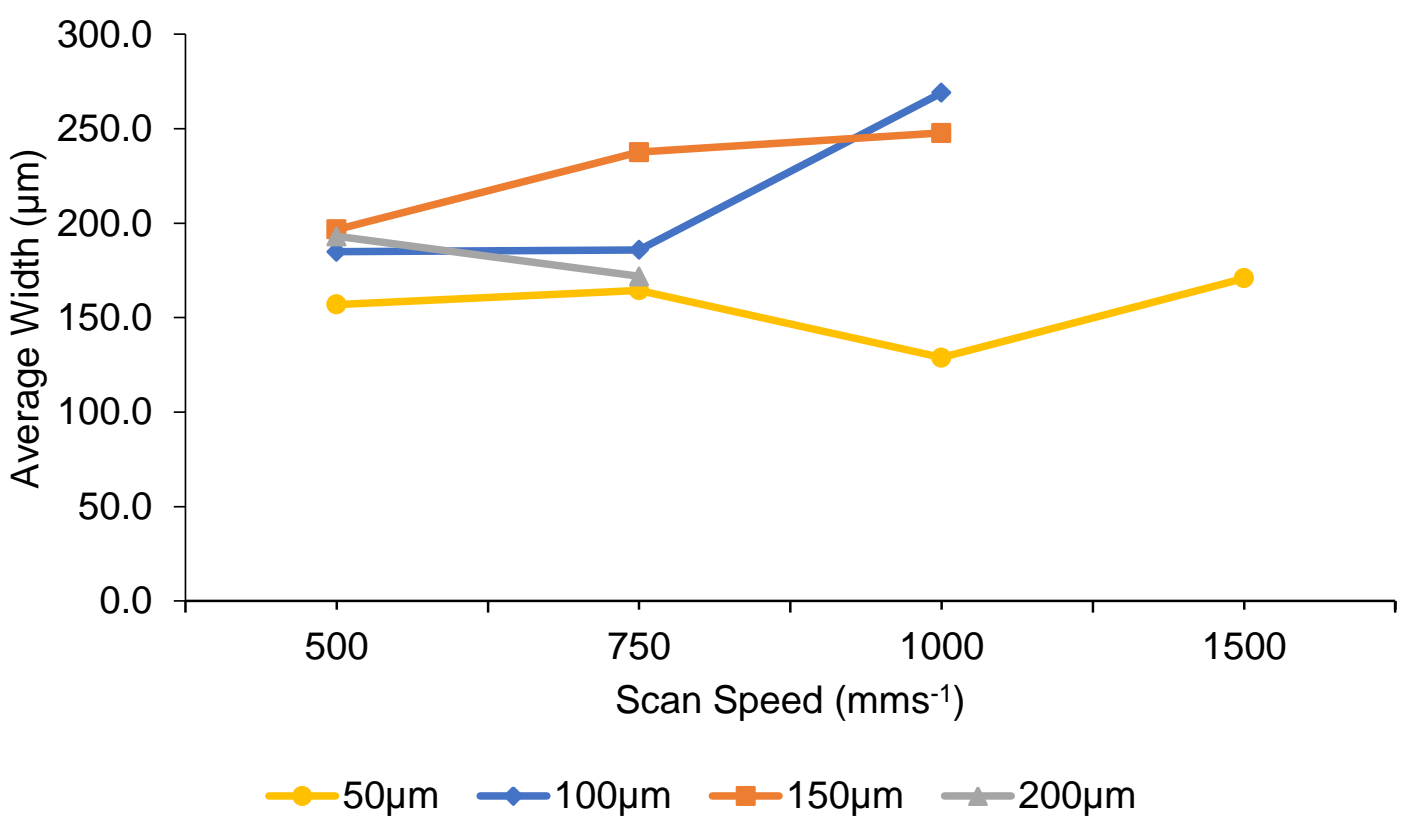

Figure 84. Experiment E (Ti-6Al-4V) - Average Track Width at 200W. 


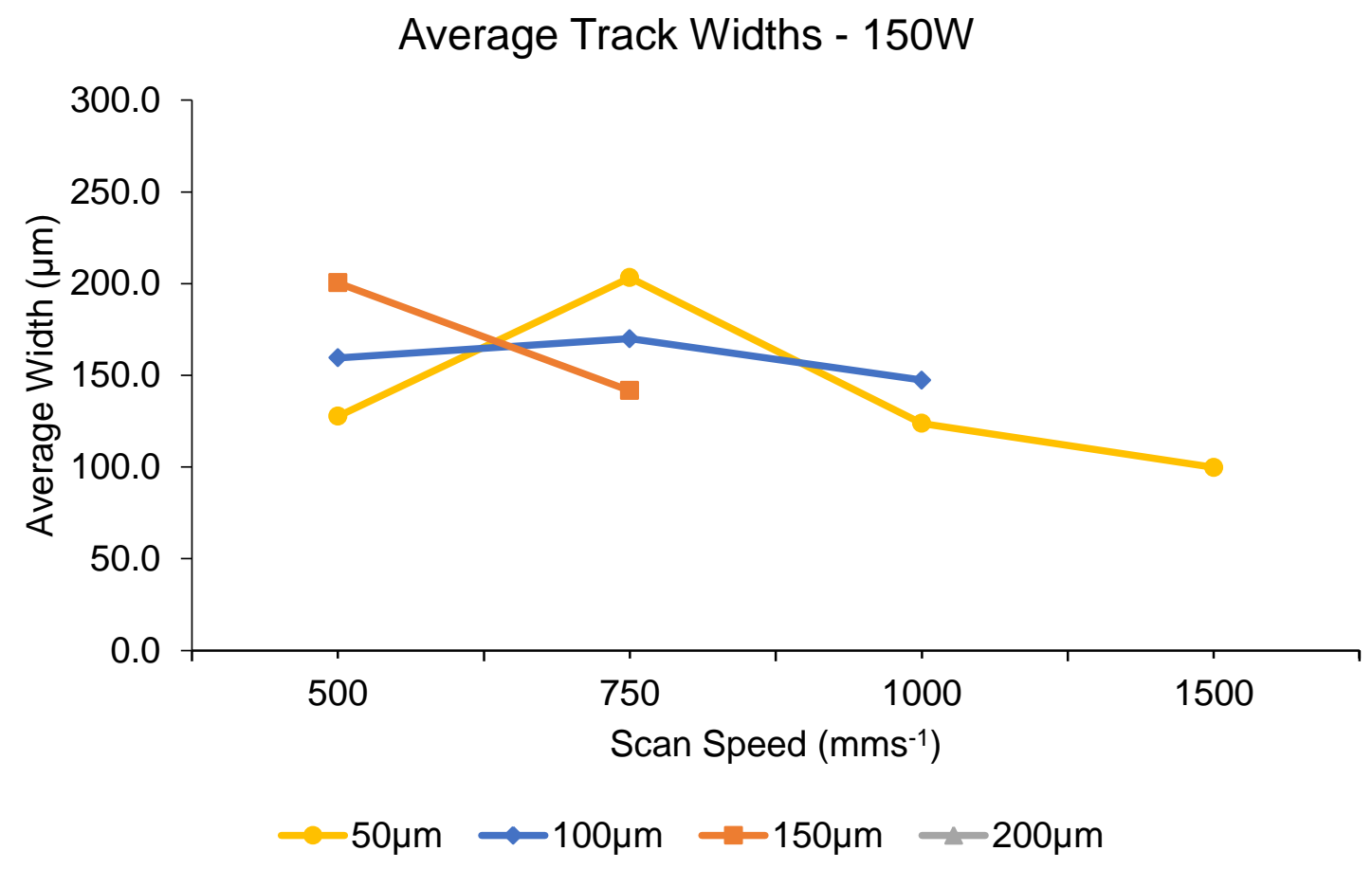

Figure 85. Experiment E (Ti-6Al-4V) - Average Track Width at $150 \mathrm{~W}$.

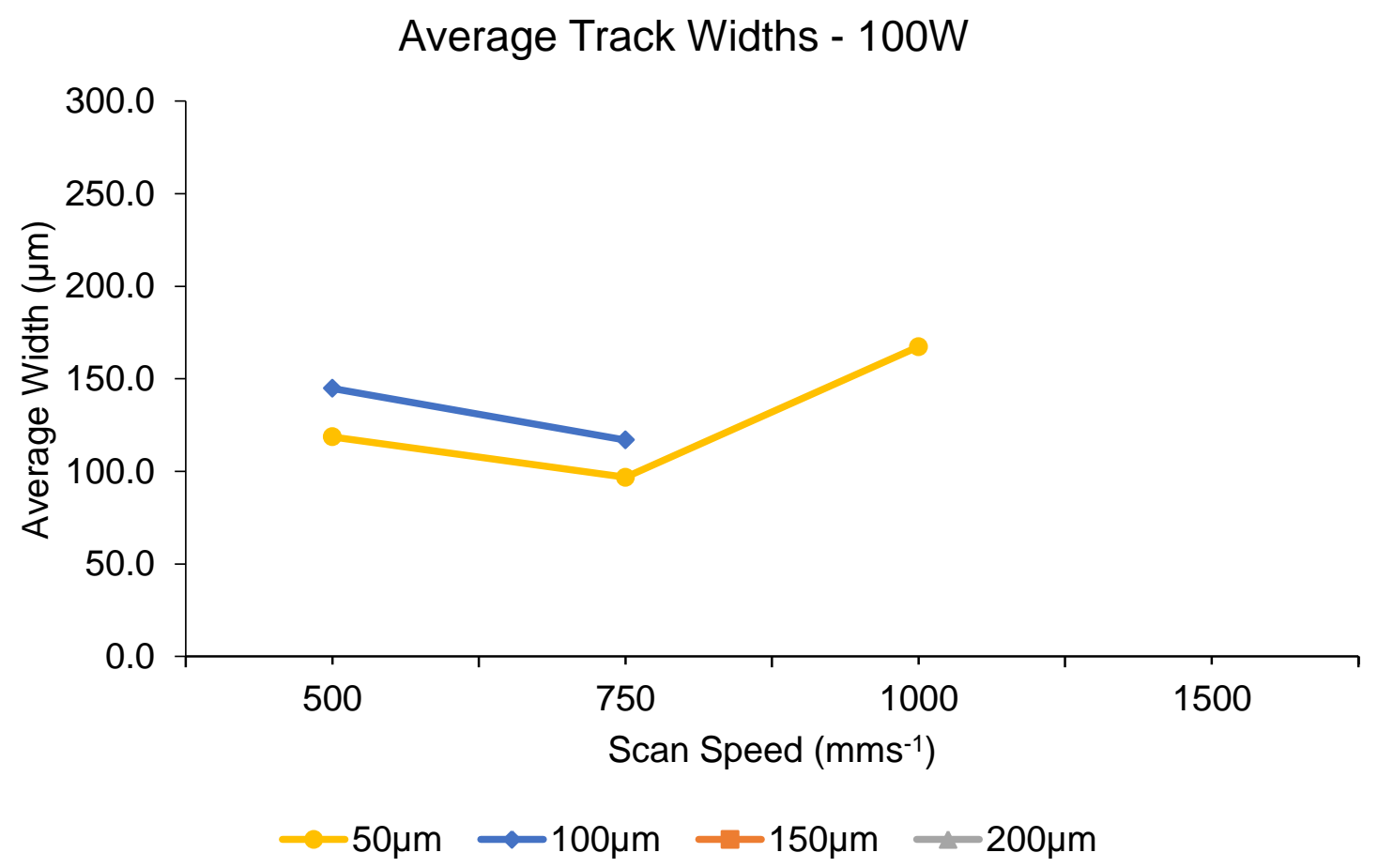

Figure 86. Experiment E (Ti-6Al-4V) - Average Track Width at $100 \mathrm{~W}$. 


\subsubsection{Conclusions}

In this chapter, the crucible method was used to successfully create single track process maps for a new material, Ti-6Al-4V titanium alloy powder. Three process parameters were investigated, the laser power, scan speed and layer thickness. The changes in the crosssectional track geometry and line stability in relation to these parameters was investigated. It was found that track stability and geometry was greatly affected by the layer thickness used during the experiment. Increasing layer thickness would increase track instability, and was seen as generally detrimental to track formation. At the highest laser power, 200W, and lowest scan speed, $100 \mathrm{mms}^{-1}$, the line build percentage at $100 \mu \mathrm{m}$ layer depth reached 93.6\%. The track was irregularly shaped but continuous, suggesting that with further optimisation of the process parameters, track stability in this region could be achieved. The DOE optimal parameters obtained from the DOE at the beginning of the chapter complimented the results obtained from this experiment. Single-tracks fabricated using nearsimilar process parameters produced continuous tracks with no sign of keyhole porosity, which is beneficial to optimising bulk density. 


\section{Chapter 6 Discussion}

Several experiments have been performed in which the process parameters were varied in order to investigate the response in the formation of either single track or single layer builds. Three principle parameters were investigated, which were laser power (W), scan speed

$\left(\mathrm{mms}^{-1}\right)$ and layer thickness $(\mu \mathrm{m})$. Two different material powders were used, stainless steel 316 $\mathrm{L}$ and titanium alloy $\mathrm{Ti}-6 \mathrm{Al}-4 \mathrm{~V}$, which reacted differently during their respective experiments, however the behavioural trends observed between experiments and materials remained consistent.

\section{Process Maps}

The cross-sectional analysis of the single tracks built in the experiments would fall under three different and distinct geometries, as seen in Figure 87, Figure 89 and Figure 90 below. During topographical examination of the tracks, the transition between stable to unstable track formation was divided into five regions, as seen in Figure 88.

The left-most image in each cross-sectional figure shows the geometry of a typical balled track. This geometry would be observed at regions of low laser power, high scan speed, or high layer depth, where the track would break down into a series of isolated droplets. This was attributed to the low temperatures achieved in the melt causing insufficient melting of the surrounding material and insufficient wetting, which causes the melt to take the shape of a sphere to reduce surface energy. Plateau-Rayleigh forces and balling would force the molten pool to from spherical droplets as a way to reduce overall surface energy. The topographical representation of such geometries can be seen in Figure 88iii-iv.

The middle image in each cross-sectional figure show what is considered the ideal geometry for fabricating structures in the laser-powder bed fusion process, often associated with conduction-mode melting. The track forms with a rounded top, with a small or medium sized penetration into the previous layers. Tracks found with this geometry would form as continuous single lines with no gaps in between solidified material, as seen in Figure 88i-ii. 
The temperatures achieved at the melt in these tracks would have been sufficient to cause melting of the powder and remelting of the previous layers, and the melt itself would have suitable wetting and spreading properties. The sufficient wetting of the melt plays a crucial role in forming an adhesive bond between the liquid and solids, as well as cohesive bonds between successive melt pools which form the track. This type of geometry and track formation is ideal for fabricating parts with optimal physical properties and reduced porosity. The right-most image in each cross-sectional figure shows a track that forms from keyholemode melting. This geometry was observed at regions of exceptionally high laser powder or low scan speed. These tracks would form as continuous single lines, similar to Figure 88 i-ii. High energy density would cause the material to reach boiling point, causing vaporisation. The recoil pressure from the vapour exerts a force onto the molten pool, causing a cavity to form within the melt. The laser achieves deeper penetration, causing the melt pool to penetrate into previous layer and form a deep, V-shaped penetration. Keyhole-mode melting can be detrimental to the laser-powder bed fusion process, as gas can become trapped within during the melt, which can cause pores to form throughout the track, [119], [125]. Two clear examples of such void formation can be seen on the right-hand images of Figure 87 and Figure 90.
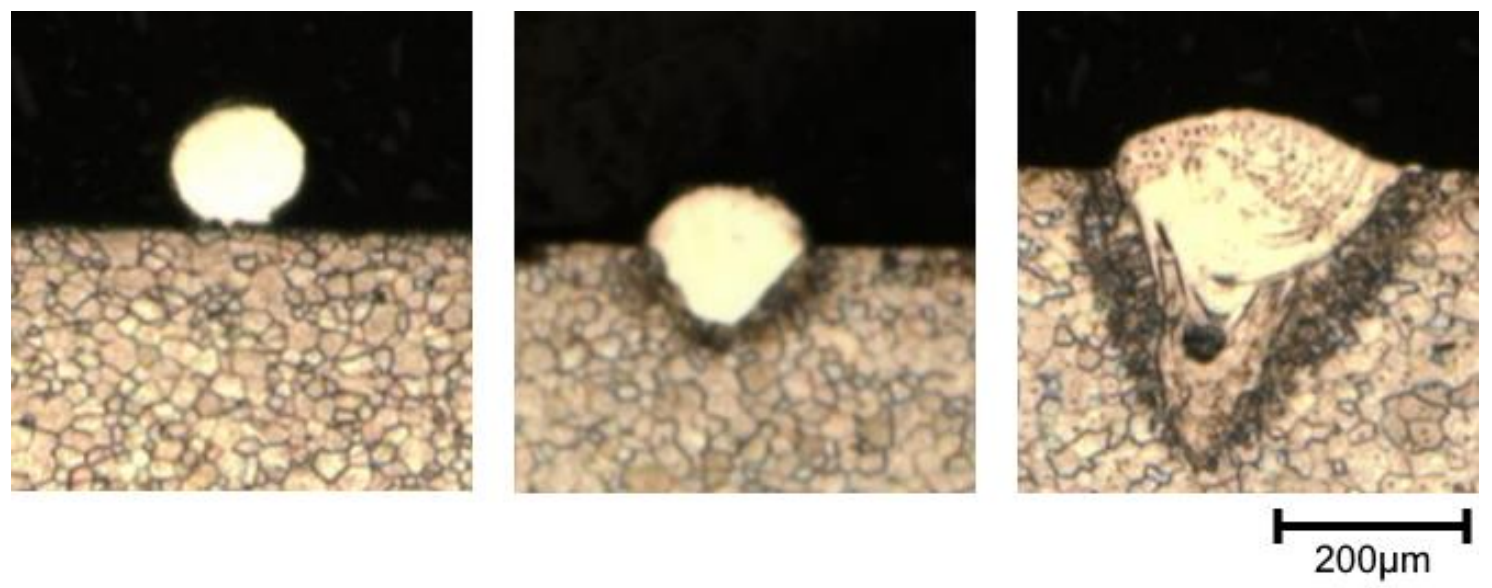

Figure 87. Transition of the track geometry in Experiment B (SS316L). Left) $125 \mathrm{~W}, 600 \mathrm{mms}^{-1}$, Middle) 200W, $300 \mathrm{mms}^{-1}$, Right) $150 \mathrm{~W}, 500 \mathrm{mms}^{-1}$ 

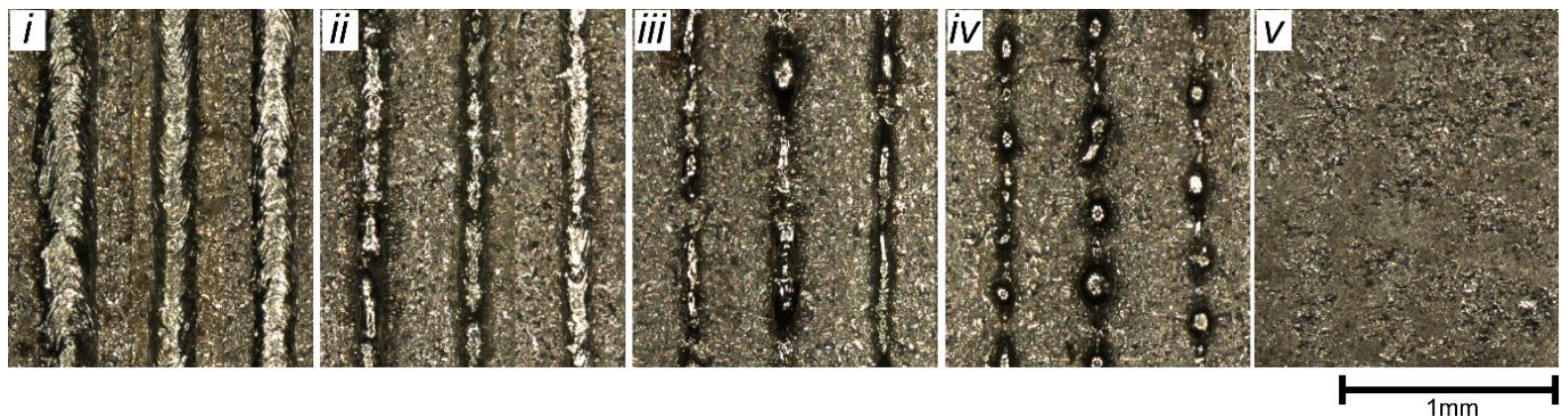

Figure 88. The five types of tracks that formed during Experiment B (SS316L).

From left to right: i) continuous/regular tracks (200W, $\left.100 \mathrm{mms}^{-1}\right)$, ii)

continuous/irregular tracks $\left(150 \mathrm{~W}, 400 \mathrm{mms}^{-1}\right)$, iii) discontinuous/irregular tracks $\left(150 \mathrm{~W}, 400 \mathrm{mms}^{-1}\right)$, iv) balling $\left.\left(175 \mathrm{~W}, 700 \mathrm{mms}^{-1}\right), \mathrm{v}\right)$ build failure $\left(100 \mathrm{~W}, 1000 \mathrm{mms}^{-1}\right)$.

The images from both the topographical and cross-sectional analysis from each single-track experiment have been compiled into several process maps, as a means of visually describing the forms of transitions that take place during melt pool formation and solidification. Additionally, the physical measurements taken from both sets of analyses have been used to show the relationships between the processing parameters and track geometry.
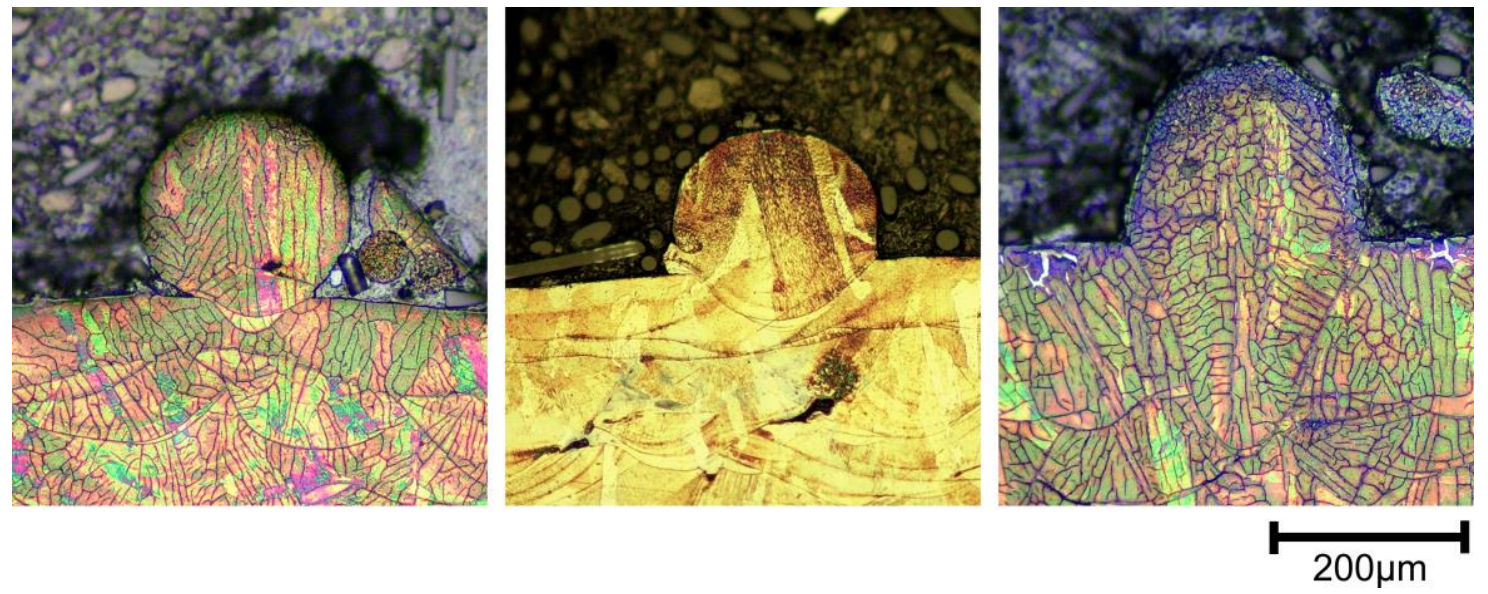

Figure 89. Transition of the track geometry in Experiment D (SS316L). Left) $200 \mathrm{~W}, 700 \mathrm{mms}^{-1}$, Middle) $150 \mathrm{~W}, 300 \mathrm{mms}^{-1}$, Right) $175 \mathrm{~W}, 200 \mathrm{mms}^{-1}$. 

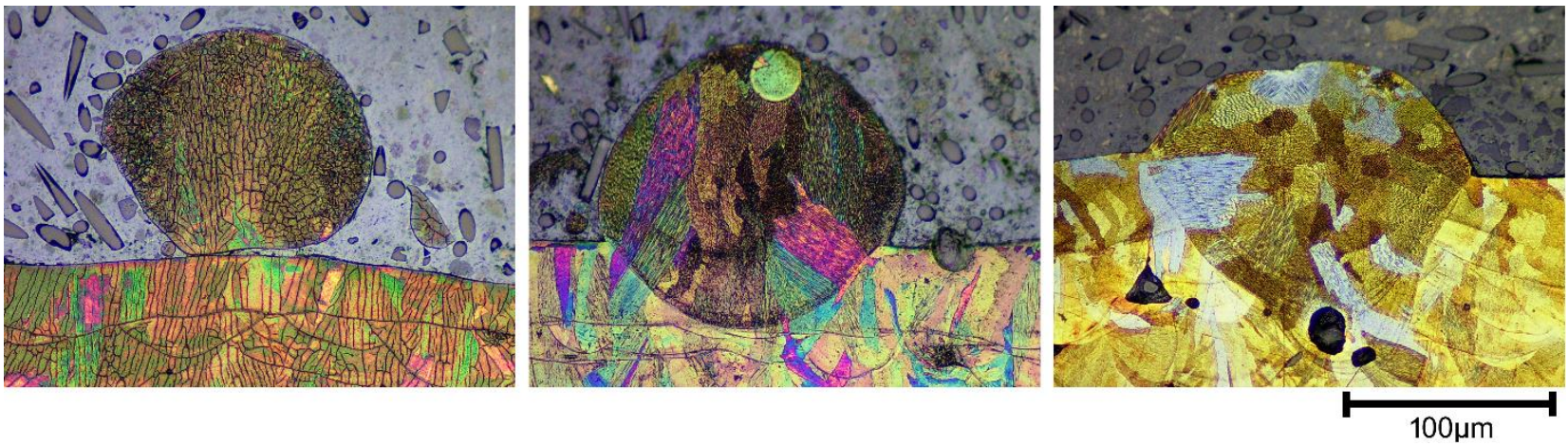

Figure 90. Transition of the track geometry in Experiment C (SS316L). Left) $200 \mathrm{~W}, 700 \mathrm{mms}^{-1}$, Middle) $150 \mathrm{~W}, 300 \mathrm{mms}^{-1}$, Right) $175 \mathrm{~W}, 200 \mathrm{mms}^{-1}$.

\section{Microstructures of the Single Track}

During track formation, a distinct pattern of solidification of the grains was routinely observed. Cellular, fine grains were observed at regions of fast cooling, that is to say, the outer edges or boundaries of the melt pool. The grain growth around these finer structures was usually columnar, growing towards the direction of the last place to cool in the melt, that is to say, the centre of the bead. These mechanisms can be observed in most of the melt beads presented in Figure 89 and Figure 90.

\section{Keyholing}

The degree of keyholing observed during each experiment was relatively minimal, due to the limitations in equipment, as laser power was restricted to 200W. Even with exceptionally low scan speeds, such as the parameters used in Experiment $\mathrm{C}$, the amount of keyhole porosity observed was relatively low and uncommon. Many contemporary laser-powder bed fusion machines are utilising laser systems that exceed the power used in this experiment, ranging from $400 \mathrm{~W}$ to several $\mathrm{kW}$. If the transition into keyhole-mode melting is achieved faster at high laser powers it can become more difficult to control. The vapourisation of the melt pool not only has detrimental effects on the physical properties of the part, but the resulting plasma/metal vapour plume can cause a number of issues. It can interact directly with the laser, causing damping and scattering of the beam, [126]-[128]. The nanoscale condensate that forms after the plume cools down is mostly removed from the powder bed by high velocity shielding gas. However, there are still chances that it can contaminate the powder 
bed, which promotes metallurgical defects to form in the part, [129], [130], and due to its extremely small size, typically less than $1 \mu \mathrm{m}$ in size, it can coat crucial components such as the lens and makes the cleaning of the build chamber and filtration systems more frequently necessary.

\section{The Crucible Design}

As an attempt to emulate the true conditions of melt pool formation and solidification during the laser-powder bed fusion process, experiments $C, D$ and $E$ utilised a new substrate design which could be constructed during the same build cycle as the single-track experiment. Most research involving single track formations use a plane surface, such as commercially available, hot rolled metal plates, as a substrate for generating single tracks, [161]-[163].

At in-situ conditions during the laser-powder bed fusion process, single track lines were constructed onto substrates made during the same build cycle, which were called crucibles. The crucible design was small and very easy to manufacture, resembling a $10 \mathrm{~mm} \times 15 \mathrm{~mm} \times$ $5 \mathrm{~mm}$ rectangular shape. They could be built very quickly, and could be used to populate an entire build plate, allowing for dozens of different combinations to be used during a single build. The crucibles could easily be removed from the plate. Additionally, the crucible design included a cavity which could have the layer depth effectively varied. This meant that the layer depth used to fabricate tracks could be included as a process parameter very easily. Two experiments were performed to determine the differences incurred by using the crucible design, Experiment B and D. In experiment D, single tracks were constructed onto mild steel inserts and were subject to topographical and metallographic investigation. The same exact parameters from Experiment B were used to create single tracks on crucible substrates in Experiment D.

It was found that tracks constructed on crucible substrates would form with less track fragmentation and balling than tracks constructed on the mild steel inserts. Regions within the process map where track failed to build on inserts were found to have increased track 
formation on crucibles, though this improvement could be very limited. This has been attributed to the presence of oxides on the mild steel substrates reducing wettability of the melt, and the increase in surface roughness by comparison to the insert surfaces. Additive manufacturing tends to produce uneven, wave-like surfaces, which can improve the wetting ability of melts that form on such surfaces, [117], [118].

Keyhole mode melting was observed less frequently, and the depth-to-width ratios for tracks made using crucibles were far lower than for those made using the inserts. However, smooth track formation was discouraged when using the crucible substrate instead of the insert substrate.

Two other experiments, experiment $\mathrm{C}$ and $\mathrm{E}$, were performed to investigate the addition of layer depth as a process parameter. It was found that increasing layer depth encouraged instability and break-up of the track. Whilst most of the experiments in this research were performed using stainless steel 316L powder, Experiment E used Ti-6Al-4V titanium alloy powder, showing that the crucible design could be successfully used with different materials. 


\section{Chapter 7 Conclusions and Further Work}

The effects of three processing parameters on the formation of single tracks during the laserpowder bed fusion process were investigated in this work. The parameters were laser power $(W)$, scan speed $\left(\mathrm{mms}^{-1}\right)$ and layer depth $(\mu \mathrm{m})$. The single tracks were analysed through topographical imaging and metallographic examination.

\subsection{Specific Conclusions}

1. A traditional single-track experiment (Experiment B), in which single-tracks are deposited directly onto a base-plate, was carried out with laser power ranging between $75 \mathrm{~W}$ and $200 \mathrm{~W}$, and with scan speed ranging between $100 \mathrm{mms}^{-1}$ and $1000 \mathrm{mms}^{-1}$, based on a point distance of $60 \mu \mathrm{m}$ and the layer depth approximately measuring $50 \mu \mathrm{m}$. Stainless steel $316 \mathrm{~L}$ metal powder was used as the building material, whilst mild steel plate was used as a substrate. It was found that:

I. Optimal track production was achieved at a laser power and scan speed of $200 \mathrm{~W}$ and $300 \mathrm{mms}^{-1}$, respectively, which should be compared to optimal settings chosen through a design of experiments based on measurements of density which gave a power of $180 \mathrm{~W}$ and a scan speed of $433 \mathrm{mms}^{-1}$. Tracks formed at this combination were continuous, remaining unbroken throughout their length, but had formed irregularities within the track.

II. Slower scan speeds yielded smoother, regular tracks, however keyhole mode melting was observed, with additional induced porosity.

III. Faster scan speeds would induce instability in the melt pool, causing track fragmentation.

2. A new experiment nominated the crucible experiment (Experiment $C$ ) was carried out with stainless steel 316 L metal powder and laser powers ranging between $100 \mathrm{~W}$ and 200W. Crucible substrate layer depths were varied between $50 \mu \mathrm{m}$ and $200 \mu \mathrm{m}$. The 
optimal parameters for smooth, continuous tracks at each layer thickness were found:

I. $50 \mu \mathrm{m}$ depth: Laser power of $100 \mathrm{~W}$ and scan speed of $87 \mathrm{mms}^{-1}$.

II. $100 \mu \mathrm{m}$ depth: Laser power of $100 \mathrm{~W}$ and scan speed of $87 \mathrm{mms}^{-1}$.

III. $150 \mu \mathrm{m}$ depth: Laser power of $130 \mathrm{~W}$ and scan speed of $113 \mathrm{mms}^{-1}$.

IV. $\quad 200 \mu \mathrm{m}$ depth: Laser power of $130 \mathrm{~W}$ and scan speed of $84 \mathrm{mms}^{-1}$.

3. A crucible experiment (Experiment D) was carried out with stainless steel 316L metal powder using the same machine parameters as used in the traditional single-track experiment $\mathrm{B}$. For this experiment, it was observed that

I. Optimal track production was achieved using a laser power and scan speed of $200 \mathrm{~W}$ and $400 \mathrm{mms}^{-1}$, respectively, which should be compared to optimal settings chosen through a design of experiments based on measurements of density which gave a power of $190 \mathrm{~W}$ and a scan speed of $500 \mathrm{mms}^{-1}$.

II. Keyhole mode melting was observed at regions of high laser power and low scan speed, with a maximum peak depth-to-width ratio of 0.6

4. A crucible experiment (Experiment E) was carried out with titanium alloy Ti-6Al-4V as the building material with laser powers ranging between $150 \mathrm{~W}$ and $200 \mathrm{~W}$, and with scan speed ranging between $500 \mathrm{mms}^{-1}$ and $3000 \mathrm{mms}^{-1}$. Crucible substrates layer depths were varied between $50 \mu \mathrm{m}$ and $200 \mu \mathrm{m}$. Using a laser power and scan speed of $200 \mathrm{~W}$ and $500 \mathrm{mms}^{-1}$, respectively, produced fairly stable, but irregular tracks at the $50 \mu \mathrm{m}$ and $100 \mu \mathrm{m}$ layer depths. At higher layer depths, tracks would fail to build or form as droplets, even at high power and low scan speeds. A larger range of processing parameters would provide more conclusive results.

5. The crucible substrate design was used successfully for three experiments. A direct comparison between single track structures made on metal-plate based substrates and the crucible substrates was carried out, and in the findings, differences were seen between the traditional single-track experiments possibly due to the surface roughness difference between the two types of substrates. 


\subsection{General Conclusions}

1. A new experiment methodology has been developed nominated the crucible experiment, in which a custom in-situ substrate is built during the same build cycle out of the same material powder. The crucible design includes an internal cavity which replicates a miniature powder bed with a variable powder layer depth. In the crucible experiment, single-tracks are fabricated within this cavity.

2. The crucible methodology has been tested on two materials (stainless steel $316 \mathrm{~L}$ and titanium alloy Ti-6Al-4V) and validated against a more traditional experiment in which single-tracks are deposited directly onto a baseplate.

3. Generally, when comparing the crucible experiments to the traditional single-track experiments, tracks were formed more readily on the crucible substrate than the traditional substrate when using the same laser processing parameters. The range in which track formation would occur would increase to include more low power and/or high scan speed combinations. The recorded line build percentage would also increase across the process map.

4. Keyhole-mode melting and pore formation was less frequently observed in the crucible method during the comparison.

5. Optimal track production was achieved using a laser power $200 \mathrm{~W}$ and higher scan speeds of $400 \mathrm{mms}^{-1}$, and were closer to the machine parameters used in practise and determined from density-based design of experiments. Tracks formed at these settings were fabricated without evidence of keyhole mode melting (less induced porosity) and had a reasonably high line build percentage (99\%).

6. The development of the crucible method has met the main objective of this work, which was to develop and standardise a single-track experimental method which would capture in a high throughput manner the instabilities and weld-profiles as a function of machine parameters. 
7. Additionally, the crucible method is more representative of the tracks laid during the process and can work for different powder alloys.

8. The capability of precisely varying the powder layer thickness onto which the single lines are build is an example of the usefulness of the method, but other parameters such as hatch spacing could also be explored.

\subsection{Further Work}

1. The baseline traditional experimentation needs to be repeated with stainless steel 316L and titanium alloy Ti-6Al-4V baseplates.

2. Crucible Experiment $D$ needs to be repeated in with a variety of layer thicknesses and with the higher power lasers now available on the REN400 and RENAM500

3. To this extent, the use of the crucible experiment on the Reduced Build Volume available on the REN400 will allow a greater exploration and optimisation of alloy composition and powder morphologies from the perspective of single tracks.

4. Crucible Experiment $E$ could also be repeated with a wider range of processing parameters (e.g. include hatch spacing and higher laser powers) to provide more conclusive results.

5. The entire set of data is now ready for comparison to computational thermal models.

6. Further analysis of transition to keyholing, particularly as a function of beam width, powder depth and using powder alloys with different powder size distributions. 


\section{References}

[1] K. Jensen, 'State-of-the-art of different available and coming RP-systems', in Proceedings of the 2nd Scandinavian Rapid Prototyping Conference, Exhibition and Course, Danish Technological Institute, Aarhus, 1993.

[2] J. P. Kruth, 'Material Incress Manufacturing by Rapid Protyping Techniques', CIRP Ann. - Manuf. Technol., vol. 40, pp. 603-614, Dec. 1991.

[3] T. T. Wohlers, W. Associates, and T. Caffrey, Wohlers Report 2014: 3D Printing and Additive Manufacturing State of the Industry Annual Worldwide Progress Report. Wohlers Associates, 2014.

[4] T. T. Wohlers and T. Caffrey, Wohlers report 2015: 3D printing and additive manufacturing state of the industry annual worldwide progress report. Fort Collins, Colo.: Wohlers Associates, 2015.

[5] G. N. Levy, R. Schindel, P. Schleiss, F. Micari, and L. Fratini, 'On the use of SLS Tools in Sheet Metal Stamping', CIRP Ann., vol. 52, no. 1, pp. 249-252, Jan. 2003.

[6] P. Stoll, A. Spierings, K. Wegener, and et al, 'SLM processing of $14 \mathrm{Ni}$ (200 Grade) maraging steel', in 3rd Fraunhofer Direct Digital Manufacturing Conference, DDMC 2016. Proceedings, 2016, p. 6.

[7] M. Gebauer, B. Müller, A. Spierings, and et al, 'High performance sheet metal forming tooling by additive manufacturing', in 6th International Conference on Additive Technologies, iCAT 2016. Proceedings, 2016, pp. 354-361.

[8] D. D. Gu, W. Meiners, K. Wissenbach, and R. Poprawe, 'Laser additive manufacturing of metallic components: materials, processes and mechanisms', Int. Mater. Rev., vol. 57, no. 3, pp. 133-164, 2012.

[9] R. H. Morgan, A. J. Papworth, C. Sutcliffe, P. Fox, and W. O'neill, 'High density net shape components by direct laser re-melting of single-phase powders', J. Mater. Sci., vol. 37, no. 15, pp. 3093-3100, Aug. 2002.

[10] K.-U. Bletzinger and E. Ramm, 'Structural optimization and form finding of light weight structures', Comput. Struct., vol. 79, no. 22, pp. 2053-2062, Sep. 2001.

[11] 'Siemens successfully tests 3D printed gas turbine blades', The Engineer, 06-Feb2017. .

[12] O. L. A. Harrysson and D. R. Cormier, 'Direct Fabrication of Custom Orthopedic Implants Using Electron Beam Melting Technology', in Advanced Manufacturing Technology for Medical Applications, I. G. Associateessor, Ed. John Wiley \& Sons, Ltd, 2005, pp. 191-206.

[13] E. Santos, K. Osakada, M. Shiomi, M. Morita, and F. Abe, 'Fabrication of titanium dental implants by selective laser melting', presented at the Fifth International Symposium on Laser Precision Microfabrication, 2004, vol. 5662, pp. 268-274.

[14] C. M. Haslauer, J. C. Springer, O. L. A. Harrysson, E. G. Loboa, N. A. Monteiro-Riviere, and D. J. Marcellin-Little, 'In vitro biocompatibility of titanium alloy discs made using direct metal fabrication', Med. Eng. Phys., vol. 32, no. 6, pp. 645-652, Jul. 2010.

[15] L. E. Murr et al., 'Microstructures and mechanical properties of electron beam-rapid manufactured Ti-6Al-4V biomedical prototypes compared to wrought Ti-6Al-4V', Mater. Charact., vol. 60, no. 2, pp. 96-105, Feb. 2009.

[16] V. Bhavar, P. Kattire, V. Patil, S. Khot, K. Gujar, and R. Singh, 'A review on powder bed fusion technology of metal additive manufacturing', in 4th International Conference and Exhibition on Additive Manufacturing Technologies-AM-2014, September, 2014, pp. 12.

[17] P. Lott, H. Schleifenbaum, W. Meiners, K. Wissenbach, C. Hinke, and J. Bültmann, 'Design of an Optical system for the In Situ Process Monitoring of Selective Laser Melting (SLM)', Phys. Procedia, vol. 12, no. Part A, pp. 683-690, Jan. 2011. 
[18] O. Rehme and C. Emmelmann, 'Reproducibility for properties of selective laser melting products', in Proceedings of the Third International WLT-Conference on Lasers in Manufacturing, Munich, 2005, pp. 1-6.

[19] H. Attar, M. Calin, L. C. Zhang, S. Scudino, and J. Eckert, 'Manufacture by selective laser melting and mechanical behavior of commercially pure titanium', Mater. Sci. Eng. A, vol. 593, pp. 170-177, Jan. 2014.

[20] X. Zhou, X. Liu, D. Zhang, Z. Shen, and W. Liu, 'Balling phenomena in selective laser melted tungsten', J. Mater. Process. Technol., vol. 222, no. Supplement C, pp. 33-42, Aug. 2015.

[21] J. P. Kruth, L. Froyen, J. Van Vaerenbergh, P. Mercelis, M. Rombouts, and B. Lauwers, 'Selective laser melting of iron-based powder', J. Mater. Process. Technol., vol. 149, no. 1, pp. 616-622, Jun. 2004.

[22] Y. Liu, Y. Yang, and D. Wang, 'A study on the residual stress during selective laser melting (SLM) of metallic powder', Int. J. Adv. Manuf. Technol., vol. 87, no. 1-4, pp. 647-656, Oct. 2016.

[23] W. Di, Y. Yongqiang, S. Xubin, and C. Yonghua, 'Study on energy input and its influences on single-track,multi-track, and multi-layer in SLM', Int. J. Adv. Manuf. Technol., vol. 58, no. 9-12, pp. 1189-1199, Feb. 2012.

[24] S. M. Gaytan, L. E. Murr, F. Medina, E. Martinez, M. I. Lopez, and R. B. Wicker, 'Advanced metal powder based manufacturing of complex components by electron beam melting', Mater. Technol., vol. 24, no. 3, pp. 180-190, Sep. 2009.

[25] M. Van Elsen, 'Complexity of selective laser melting : a new optimisation approach', Jan. 2007.

[26] W. E. King et al., 'Observation of keyhole-mode laser melting in laser powder-bed fusion additive manufacturing', J. Mater. Process. Technol., vol. 214, no. 12, pp. 29152925, Dec. 2014.

[27] M. Zhang, G. Chen, Y. Zhou, and S. Li, 'Direct observation of keyhole characteristics in deep penetration laser welding with a 10 kW fiber laser', Opt. Express, vol. 21, no. 17, pp. 19997-20004, Aug. 2013.

[28] E. Assuncao, S. Williams, and D. Yapp, 'Interaction time and beam diameter effects on the conduction mode limit', Opt. Lasers Eng., vol. 50, no. 6, pp. 823-828, Jun. 2012.

[29] I. Yadroitsev, A. Gusarov, I. Yadroitsava, and I. Smurov, 'Single track formation in selective laser melting of metal powders', J. Mater. Process. Technol., vol. 210, no. 12, pp. 1624-1631, 2010.

[30] I. Yadroitsev and I. Smurov, 'Selective laser melting technology: From the single laser melted track stability to 3D parts of complex shape', Phys. Procedia, vol. 5, pp. 551560, Jan. 2010.

[31] U. Scipioni Bertoli, A. J. Wolfer, M. J. Matthews, J.-P. R. Delplanque, and J. M. Schoenung, 'On the limitations of Volumetric Energy Density as a design parameter for Selective Laser Melting', Mater. Des., vol. 113, pp. 331-340, Jan. 2017.

[32] H. Gong et al., 'Melt pool characterization for selective laser melting of Ti-6Al-4V prealloyed powder', in Solid freeform fabrication symposium, 2014, pp. 256-267.

[33] ASTM International, Standard terminology for additive manufacturing technologies: designation F2792-12a. West Conshohocken, PA: ASTM International, 2012.

[34] D. Cormier, O. Harrysson, T. Mahale, and H. West, 'Freeform Fabrication of Titanium Aluminide via Electron Beam Melting Using Prealloyed and Blended Powders', Advances in Materials Science and Engineering, 2007. [Online]. Available: https://www.hindawi.com/journals/amse/2007/034737/abs/. [Accessed: 28-Nov-2017].

[35] M. Koike et al., 'Evaluation of Titanium Alloys Fabricated Using Rapid Prototyping Technologies-Electron Beam Melting and Laser Beam Melting', Materials, vol. 4, no. 10, pp. 1776-1792, Oct. 2011.

[36] K. Taminger and R. A. Hafley, 'Characterization of 2219 aluminum produced by electron beam freeform fabrication', 2002. 
[37] S. M. Gaytan et al., 'Comparison of Microstructures and Mechanical Properties for Solid and Mesh Cobalt-Base Alloy Prototypes Fabricated by Electron Beam Melting', Metall. Mater. Trans. A, vol. 41, no. 12, pp. 3216-3227, Dec. 2010.

[38] D. Cormier, O. Harrysson, and $\mathrm{H}$. West, 'Characterization of $\mathrm{H} 13$ steel produced via electron beam melting', Rapid Prototyp. J., vol. 10, no. 1, pp. 35-41, Feb. 2004.

[39] P. Frigola et al., 'Fabricating copper components with electron beam melting', Adv. Mater. Process., vol. 172, no. 7, pp. 20-24, Jan. 2014.

[40] L. E. Murr et al., 'Metal fabrication by additive manufacturing using laser and electron beam melting technologies', J. Mater. Sci. Technol., vol. 28, no. 1, pp. 1-14, 2012.

[41] S. M. Gaytan et al., 'Comparison of microstructures and mechanical properties for solid cobalt-base alloy components and biomedical implant prototypes fabricated by electron beam melting', in Proceedings of Solid Freeform Fabrication (SFF) Symposium, Austin, TX, USA, 2010.

[42] D. Cormier, H. West, O. Harrysson, and K. Knowlson, 'Characterization of thin walled Ti-6Al-4V components produced via electron beam melting', in Solid freeform fabrication symposium, 2004, pp. 2-4.

[43] S. Biamino et al., 'Electron beam melting of Ti-48Al-2Cr-2Nb alloy: Microstructure and mechanical properties investigation', Intermetallics, vol. 19, no. 6, pp. 776-781, Jun. 2011.

[44] G. Baudana et al., 'Titanium aluminides for aerospace and automotive applications processed by Electron Beam Melting: Contribution of Politecnico di Torino', Met. Powder Rep., vol. 71, no. 3, pp. 193-199, May 2016.

[45] P. Heinl, L. Müller, C. Körner, R. F. Singer, and F. A. Müller, 'Cellular Ti-6Al-4V structures with interconnected macro porosity for bone implants fabricated by selective electron beam melting', Acta Biomater., vol. 4, no. 5, pp. 1536-1544, Sep. 2008.

[46] X. Li, C. Wang, W. Zhang, and Y. Li, 'Fabrication and characterization of porous Ti6Al4V parts for biomedical applications using electron beam melting process', Mater. Lett., vol. 63, no. 3, pp. 403-405, Feb. 2009.

[47] M. Koike, K. Martinez, L. Guo, G. Chahine, R. Kovacevic, and T. Okabe, 'Evaluation of titanium alloy fabricated using electron beam melting system for dental applications', $J$. Mater. Process. Technol., vol. 211, no. 8, pp. 1400-1408, Aug. 2011.

[48] C. Körner, 'Additive manufacturing of metallic components by selective electron beam melting - a review', Int. Mater. Rev., vol. 61, no. 5, pp. 361-377, Jul. 2016.

[49] J. Milberg and M. Sigl, 'Electron beam sintering of metal powder', Prod. Eng., vol. 2, no. 2, pp. 117-122, Jun. 2008.

[50] K. Taminger and R. A. Hafley, 'Electron beam freeform fabrication: a rapid metal deposition process', Proc. 3rd Annu. Automot. Compos. Conf., 2003.

[51] M. L. Griffith et al., 'Free form fabrication of metallic components using laser engineered net shaping (LENS)', in Solid Freeform Fabrication Proceedings, 1996, vol. 9 , pp. $125-131$.

[52] J. Mazumder, D. Dutta, N. Kikuchi, and A. Ghosh, 'Closed loop direct metal deposition: Art to Part', Opt. Lasers Eng., vol. 34, no. 4-6, pp. 397-414, 2000.

[53] Y. Li, X. Huang, Y. Liu, H. Peng, and M. Azer, 'Laser net shape manufacturing of metallic materials with $\mathrm{CO} 2$ and fiber laser', 24th Int. Congr. Appl. Lasers Electro-Opt. ICALEO 2005 - Congr. Proc., pp. 320-325, Jan. 2005.

[54] S. Nowotny, S. Scharek, F. Kempe, and et al, 'COAXn: Modular system of powder nozzles for laser beam build-up welding', in ICALEO 2003, 22nd International Congress on Applications of Lasers and Electro Optics. Congress proceedings. CD-ROM, 2003, p. Paper P519.

[55] M. S. Domack and J. M. Baughman, 'Development of nickel-titanium graded composition components', Rapid Prototyp. J., vol. 11, no. 1, pp. 41-51, 2005.

[56] L. Bian, S. M. Thompson, and N. Shamsaei, 'Mechanical Properties and Microstructural Features of Direct Laser-Deposited Ti-6Al-4V', JOM, vol. 67, no. 3, pp. 629-638, Mar. 2015. 
[57] B. Dutta, V. Singh, H. Natu, J. Choi, and J. Mazumder, 'Direct metal deposition', Adv Mater Process, vol. 167, pp. 29-31, 2009.

[58] C. C. Ng, M. M. Savalani, M. L. Lau, and H. C. Man, 'Microstructure and mechanical properties of selective laser melted magnesium', Appl. Surf. Sci., vol. 257, no. 17, pp. 7447-7454, Jun. 2011.

[59] K. G. Prashanth et al., 'Microstructure and mechanical properties of Al-12Si produced by selective laser melting: Effect of heat treatment', Mater. Sci. Eng. A, vol. 590, pp. 153-160, Jan. 2014.

[60] D. Kaminski, 'LASER MARKING: How to choose the best laser for your marking application'. [Online]. Available: http://www.laserfocusworld.com/articles/2011/04/lasermarking-how-to-choose-the-best-laser-for-your-marking-application.html. [Accessed: 10-Mar-2017].

[61] J. C. Maxwell, 'VIII. A dynamical theory of the electromagnetic field', Philos. Trans. $R$. Soc. Lond., vol. 155, pp. 459-512, Jan. 1865.

[62] A. Einstein, 'On the electrodynamics of moving bodies', 1905.

[63] 'How a Laser Works', Environmental Health and Safety, 09-Oct-2009. [Online]. Available: http://ehs.oregonstate.edu/laser/training/how-laser-works. [Accessed: 04Oct-2017].

[64] J.-P. Kruth, P. Mercelis, J. Van Vaerenbergh, L. Froyen, and M. Rombouts, 'Binding mechanisms in selective laser sintering and selective laser melting', Rapid Prototyp. J., vol. 11, no. 1, pp. 26-36, 2005.

[65] H. J. Niu and I. T. H. Chang, 'Instability of scan tracks of selective laser sintering of high speed steel powder', Scr. Mater., vol. 41, no. 11, pp. 1229-1234, 1999.

[66] P. Mercelis and J. Kruth, 'Residual stresses in selective laser sintering and selective laser melting', Rapid Prototyp. J., vol. 12, no. 5, pp. 254-265, Oct. 2006.

[67] P. Mercelis, Control of Selective Laser Sintering an Selective Laser Melting Processes. Katholieke Universiteit te Leuven (1970- ), 2007.

[68] T. Furumoto, T. Ueda, A. Aziz, M. Sanusi, A. Hosokawa, and R. Tanaka, 'Study on reduction of residual stress induced during rapid tooling process: Influence of heating conditions on residual stress', in Key Engineering Materials, 2010, vol. 447, pp. 785789.

[69] H. Gong, K. Rafi, H. Gu, T. Starr, and B. Stucker, 'Analysis of defect generation in Ti$6 \mathrm{Al}-4 \mathrm{~V}$ parts made using powder bed fusion additive manufacturing processes', Addit. Manuf., vol. 1-4, pp. 87-98, Oct. 2014.

[70] M. Cloots, P. J. Uggowitzer, and K. Wegener, 'Investigations on the microstructure and crack formation of IN738LC samples processed by selective laser melting using Gaussian and doughnut profiles', Mater. Des., vol. 89, pp. 770-784, Jan. 2016.

[71] N. K. Tolochko et al., 'Balling processes during selective laser treatment of powders', Rapid Prototyp. J., vol. 10, no. 2, pp. 78-87, Apr. 2004.

[72] M. Khan and P. Dickens, 'Selective Laser Melting (SLM) of pure gold', Gold Bull., vol. 43, no. 2, pp. 114-121, 2010.

[73] R. Li, J. Liu, Y. Shi, L. Wang, and W. Jiang, 'Balling behavior of stainless steel and nickel powder during selective laser melting process', Int. J. Adv. Manuf. Technol., vol. 59, no. 9-12, pp. 1025-1035, Apr. 2012.

[74] I. Yadroitsev, P. Krakhmalev, I. Yadroitsava, S. Johansson, and I. Smurov, 'Energy input effect on morphology and microstructure of selective laser melting single track from metallic powder', J. Mater. Process. Technol., vol. 213, no. 4, pp. 606-613, Apr. 2013.

[75] W. J. Sames, F. A. List, S. Pannala, R. R. Dehoff, and S. S. Babu, 'The metallurgy and processing science of metal additive manufacturing', Int. Mater. Rev., vol. 61, no. 5, pp. 315-360, Jul. 2016.

[76] R. Li, Y. Shi, Z. Wang, L. Wang, J. Liu, and W. Jiang, 'Densification behavior of gas and water atomized $316 \mathrm{~L}$ stainless steel powder during selective laser melting', Appl. Surf. Sci., vol. 256, no. 13, pp. 4350-4356, Apr. 2010.

[77] P. Karapatis, 'A sub-process approach of selective laser sintering', 2002. 
[78] 'Introduction to Additive Manufacturing Technology: A Guide for Designers and Engineers'. European Powder Metallurgy Association, 2015.

[79] A. Lawley, 'Preparation of Metal Powders', Annu. Rev. Mater. Sci., vol. 8, no. 1, pp. 49$71,1978$.

[80] K. G. Prashanth, 'Selective laser melting of Al-12Si', Nov. 2013.

[81] D. Bergstrom, J. Powell, and A. F. H. Kaplan, 'The absorptance of steels to Nd:YLF and Nd:YAG laser light at room temperature', Appl. Surf. Sci., vol. 253, pp. 5017-5028, Mar. 2007.

[82] N. K. Tolochko, Y. V. Khlopkov, S. E. Mozzharov, M. B. Ignatiev, T. Laoui, and V. I. Titov, 'Absorptance of powder materials suitable for laser sintering', Rapid Prototyp. J., vol. 6, no. 3, pp. 155-161, Sep. 2000.

[83] D. Bergström, 'The absorption of laser light by rough metal surfaces', Lulela a tekniska universitet, 2008.

[84] C. M. Taylor, T. H. C. Childs, and C. Hauser, 'Morphology of direct SLS-processed stainless steel layers', in Proceedings of the 13th Solid Solid Freeform Fabrication Symposium, Austin, 2002, pp. 530-537.

[85] P. Fischer, V. Romano, H. P. Weber, N. P. Karapatis, E. Boillat, and R. Glardon, 'Sintering of commercially pure titanium powder with a Nd:YAG laser source', Acta Mater., vol. 51, no. 6, pp. 1651-1662, Apr. 2003.

[86] J. P. Kruth, X. Wang, T. Laoui, and L. Froyen, 'Lasers and materials in selective laser sintering', Assem. Autom., vol. 23, no. 4, pp. 357-371, Dec. 2003.

[87] C. D. Boley, S. A. Khairallah, and A. M. Rubenchik, 'Calculation of laser absorption by metal powders in additive manufacturing', Appl. Opt., vol. 54, no. 9, p. 2477, Mar. 2015.

[88] I. Grattan-Guinness, 'Chapter 26 - Joseph Fourier, Théorie analytique de la chaleur (1822)', in Landmark Writings in Western Mathematics 1640-1940, Amsterdam: Elsevier Science, 2005, pp. 354-365.

[89] H. J. Niu and I. T. H. Chang, 'Liquid phase sintering of $M 3 / 2$ high speed steel by selective laser sintering', Scr. Mater., vol. 39, no. 1, pp. 67-72, 1998.

[90] J. P. Kruth, L. Froyen, M. Rombouts, J. Van Vaerenbergh, and P. Mercells, 'New Ferro Powder for Selective Laser Sintering of Dense Parts', CIRP Ann. - Manuf. Technol., vol. 52, no. 1, pp. 139-142, Jan. 2003.

[91] T. Young, 'III. An essay on the cohesion of fluids', Philos. Trans. R. Soc. Lond., vol. 95, pp. 65-87, Jan. 1805.

[92] E. O. t Olakanmi, R. F. Cochrane, and K. W. Dalgarno, 'A review on selective laser sintering/melting (SLS/SLM) of aluminium alloy powders: Processing, microstructure, and properties', Prog. Mater. Sci., vol. 74, pp. 401-477, 2015.

[93] A. Simchi, 'Direct laser sintering of metal powders: Mechanism, kinetics and microstructural features', Mater. Sci. Eng. A, vol. 428, no. 1-2, pp. 148-158, Jul. 2006.

[94] F. Klocke and C. Wagner, 'Coalescence behaviour of two metallic particles as base mechanism of selective laser sintering', CIRP Ann.-Manuf. Technol., vol. 52, no. 1, pp. 177-180, 2003.

[95] C. Hauser, T. H. C. Childs, and M. Badrossamay, 'Further developments in process mapping and modelling in direct metal selective laser melting', 15th Solid Free Form Fabr. Proc. Eds Bourell RH Al Austin Tex. August, pp. 2-4, 2004.

[96] Joseph Antoine Ferdinand Plateau, Statique expérimentale et théorique des liquides soumis aux seules forces moléculaires. Gauthier-Villars, 1873.

[97] Lord Rayleigh, 'On The Instability Of Jets', Proc. Lond. Math. Soc., vol. s1-10, no. 1, pp. 4-13, Nov. 1878.

[98] 'Plateau-Rayleigh instability', Wikipedia. 02-Aug-2017.

[99] K. C. Mills and B. J. Keene, 'Factors affecting variable weld penetration', Int. Mater. Rev., vol. 35, no. 1, pp. 185-216, 1990.

[100]R. Rai, J. W. Elmer, T. A. Palmer, and T. DebRoy, 'Heat transfer and fluid flow during keyhole mode laser welding of tantalum, Ti-6Al-4V, 304L stainless steel and vanadium', J. Phys. Appl. Phys., vol. 40, no. 18, p. 5753, 2007. 
[101]S. Pang, W. Chen, and W. Wang, A Quantitative Model of Keyhole Instability Induced Porosity in Laser Welding of Titanium Alloy, vol. 45. 2014.

[102]A. Spierings, N. Herres, and G. Levy, 'Influence of the particle size distribution on surface quality and mechanical properties in AM steel parts', Rapid Prototyp. J. RAPID Prototyp. J, vol. 17, pp. 195-202, Apr. 2011.

[103]L. Thijs, F. Verhaeghe, T. Craeghs, J. V. Humbeeck, and J.-P. Kruth, 'A study of the microstructural evolution during selective laser melting of Ti-6Al-4V', Acta Mater., vol. 58, no. 9, pp. 3303-3312, May 2010.

[104]N. P. Lavery et al., 'Effects of hot isostatic pressing on the elastic modulus and tensile properties of 316L parts made by powder bed laser fusion', Mater. Sci. Eng. A, vol. 693, no. Supplement C, pp. 186-213, May 2017.

[105]J. Schindelin, Fiji: an open-source platform for biological-image analysis, vol. 9(7). 2012.

[106]R. Asthana and N. Sobczak, 'Wettability, Spreading, and Interfacial Phenomena in High-Temperature Coatings', JOM J. Miner. Met. Mater. Soc., vol. 52, Jan. 2000.

[107]M. Agarwala, D. Bourell, J. Beaman, H. Marcus, and J. Barlow, 'Direct selective laser sintering of metals', Rapid Prototyp. J., vol. 1, no. 1, pp. 26-36, Mar. 1995.

[108]G. G. Gladush and I. Smurov, Physics of laser materials processing: theory and experiment, vol. 146. Springer Science \& Business Media, 2011.

[109]D. C. Weckman, H. W. Kerr, and J. T. Liu, 'The effects of process variables on pulsed $\mathrm{Nd}$ :YAG laser spot welds: Part II. AA 1100 aluminum and comparison to AISI 409 stainless steel', Metall. Mater. Trans. B, vol. 28, no. 4, pp. 687-700, Aug. 1997.

[110]S. Nakamura, M. Sakurai, K. Kamimuki, T. Inoue, and Y. Ito, 'Detection technique for transition between deep penetration mode and shallow penetration mode in CO 2 laser welding of metals', J. Phys. Appl. Phys., vol. 33, no. 22, p. 2941, 2000.

[111]Y. Feng, Z. Luo, Z. Liu, Y. Li, Y. Luo, and Y. Huang, 'Keyhole gas tungsten arc welding of AISI 316L stainless steel', Mater. Des., vol. 85, pp. 24-31, Nov. 2015.

[112]K.-M. Hong and Y. C. Shin, 'Analysis of microstructure and mechanical properties change in laser welding of Ti6Al4V with a multiphysics prediction model', J. Mater. Process. Technol., vol. 237, pp. 420-429, Nov. 2016.

[113]E. M. Stanciu, A. C. Păvălache, G. M. Dumitru, O. G. Dontu, D. Besnea, and I. M. Vasile, 'MECHANISM OF KEYHOLE FORMATION IN LASER WELDING', no. 38, p. 7, 2010.

[114]S. Das, 'Physical Aspects of Process Control in Selective Laser Sintering of Metals', Adv. Eng. Mater., vol. 5, no. 10, pp. 701-711, Oct. 2003.

[115]G. Strano, L. Hao, R. M. Everson, and K. E. Evans, 'Surface roughness analysis, modelling and prediction in selective laser melting', J. Mater. Process. Technol., vol. 213, no. 4, pp. 589-597, Apr. 2013.

[116]R. N. Wenzel, 'RESISTANCE OF SOLID SURFACES TO WETTING BY WATER', Ind. Eng. Chem., vol. 28, no. 8, pp. 988-994, Aug. 1936.

[117]T. Furumoto, T. Ueda, A. Hosokawa, A. Yassin, and S. Abe, 'Study on the Sintering Characteristics of the Mixed Metal Powder with Yb Fiber Laser- Evaluation of the Adhesion Force of the Sintered Material on the Different Surface Plate', J. Jpn. Soc. Precis. Eng., vol. 74, no. 8, pp. 836-840, 2008.

[118]M. R. Alkahari, T. Furumoto, T. Ueda, and A. Hosokawa, 'Consolidation characteristics of ferrous-based metal powder in additive manufacturing', J. Adv. Mech. Des. Syst. Manuf., vol. 8, no. 1, pp. JAMDSM0009-JAMDSM0009, 2014.

[119]W. E. King et al., 'Observation of keyhole-mode laser melting in laser powder-bed fusion additive manufacturing', J. Mater. Process. Technol., vol. 214, no. 12, pp. 29152925, Dec. 2014.

[120]M. Das, V. K. Balla, D. Basu, S. Bose, and A. Bandyopadhyay, 'Laser processing of SiC-particle-reinforced coating on titanium', Scr. Mater., vol. 63, no. 4, pp. 438-441, Aug. 2010. 
[121]N. E. Hodge, R. M. Ferencz, and J. M. Solberg, 'Implementation of a thermomechanical model for the simulation of selective laser melting', Comput. Mech., vol. 54, no. 1, pp. 33-51, Jul. 2014.

[122]M. S. F. de Lima and S. Sankaré, 'Microstructure and mechanical behavior of laser additive manufactured AISI 316 stainless steel stringers', Mater. Des., vol. 55, pp. 526532, Mar. 2014.

[123]C. Qiu, M. A. Kindi, A. S. Aladawi, and I. A. Hatmi, 'A comprehensive study on microstructure and tensile behaviour of a selectively laser melted stainless steel', Sci. Rep., vol. 8, no. 1, p. 7785, May 2018.

[124]D. Gu and Y. Shen, 'Balling phenomena in direct laser sintering of stainless steel powder: Metallurgical mechanisms and control methods', Mater. Des., vol. 30, no. 8, pp. 2903-2910, Sep. 2009.

[125]J. D. Madison and L. K. Aagesen, 'Quantitative characterization of porosity in laser welds of stainless steel', Scr. Mater., vol. 67, no. 9, pp. 783-786, Nov. 2012.

[126]J. Hoffman, T. Mościcki, and Z. Szymański, 'Modelling of time dependent plasma plume induced during laser welding', Czechoslov. J. Phys., vol. 56, no. 2, pp. B938B943, Oct. 2006.

[127]J. Svenungsson, I. Choquet, and A. F. H. Kaplan, 'Laser Welding Process - A Review of Keyhole Welding Modelling', Phys. Procedia, vol. 78, no. Supplement C, pp. 182191, Jan. 2015.

[128]B. Ferrar, L. Mullen, E. Jones, R. Stamp, and C. J. Sutcliffe, 'Gas flow effects on selective laser melting (SLM) manufacturing performance', J. Mater. Process. Technol., vol. 212, no. 2, pp. 355-364, Feb. 2012.

[129]C. Qiu, C. Panwisawas, M. Ward, H. C. Basoalto, J. W. Brooks, and M. M. Attallah, 'On the role of melt flow into the surface structure and porosity development during selective laser melting', Acta Mater., vol. 96, no. Supplement C, pp. 72-79, Sep. 2015.

[130]Y. Liu, Y. Yang, S. Mai, D. Wang, and C. Song, 'Investigation into spatter behavior during selective laser melting of AISI 316L stainless steel powder', Mater. Des., vol. 87, pp. 797-806, Dec. 2015.

[131]X. Shi, S. Ma, C. Liu, and Q. Wu, 'Parameter optimization for Ti-47Al-2Cr-2Nb in selective laser melting based on geometric characteristics of single scan tracks', Opt. Laser Technol., vol. 90, pp. 71-79, May 2017.

[132]Y. Guo, L. Jia, B. Kong, N. Wang, and H. Zhang, 'Single track and single layer formation in selective laser melting of niobium solid solution alloy', Chin. J. Aeronaut, Sep. 2017. 


\section{Appendix 1 - TMS Paper 3026}

\section{Verification of Numerically Calculated Cooling Rates of Powder Bed Additive Manufacturing}

H.-W. Mindt ${ }^{1}$, M. Megahed ${ }^{1}$, N.P. Lavery ${ }^{2}$, A. Giordimaina ${ }^{2}$, S.G.R. Brown ${ }^{2}$

'ESI Group, Kruppstr. 90, 45145 Essen, Germany

${ }^{2}$ Swansea University, Bay Campus, College of Engineering, Fabian Way, Crymlyn Burrows, Swansea, SA1 8EN United Kingdom

Keywords: Additive Manufacturing, Powder Bed, Direct Metal Laser Melting, Modelling, Verification

preliminary validation effort was performed by comparing the measured track widths with those predicted numerically [5] and by comparing the level of porosity obtained for different 
processing conditions [9]. In an attempt to approach code further verification the authors purse the specification of a reference benchmark for DMLM/SLM.

\section{Modelling Algorithms}

Micro-models are pursued to resolve the melt pool physics including laser radiative interaction with the powder, heat transfer, phase change and surface tension forces and Marangoni forces. They are based on computational fluid dynamics algorithms to solve the Navier-Stokes equations $[10,11,12,13]$. The momentum equations are extended using source terms to account for gravitational forces, recoil pressure and surface tension. The energy equation is complemented with source terms accounting for latent heat of fusion and evaporation as well as radiation. The laser is modelled as a Gaussian heat source. Two codes are used to solve the conservation equations, one based on the Finite Volume $[14,15,16]$ and the other on Lattice Boltzmann [17,
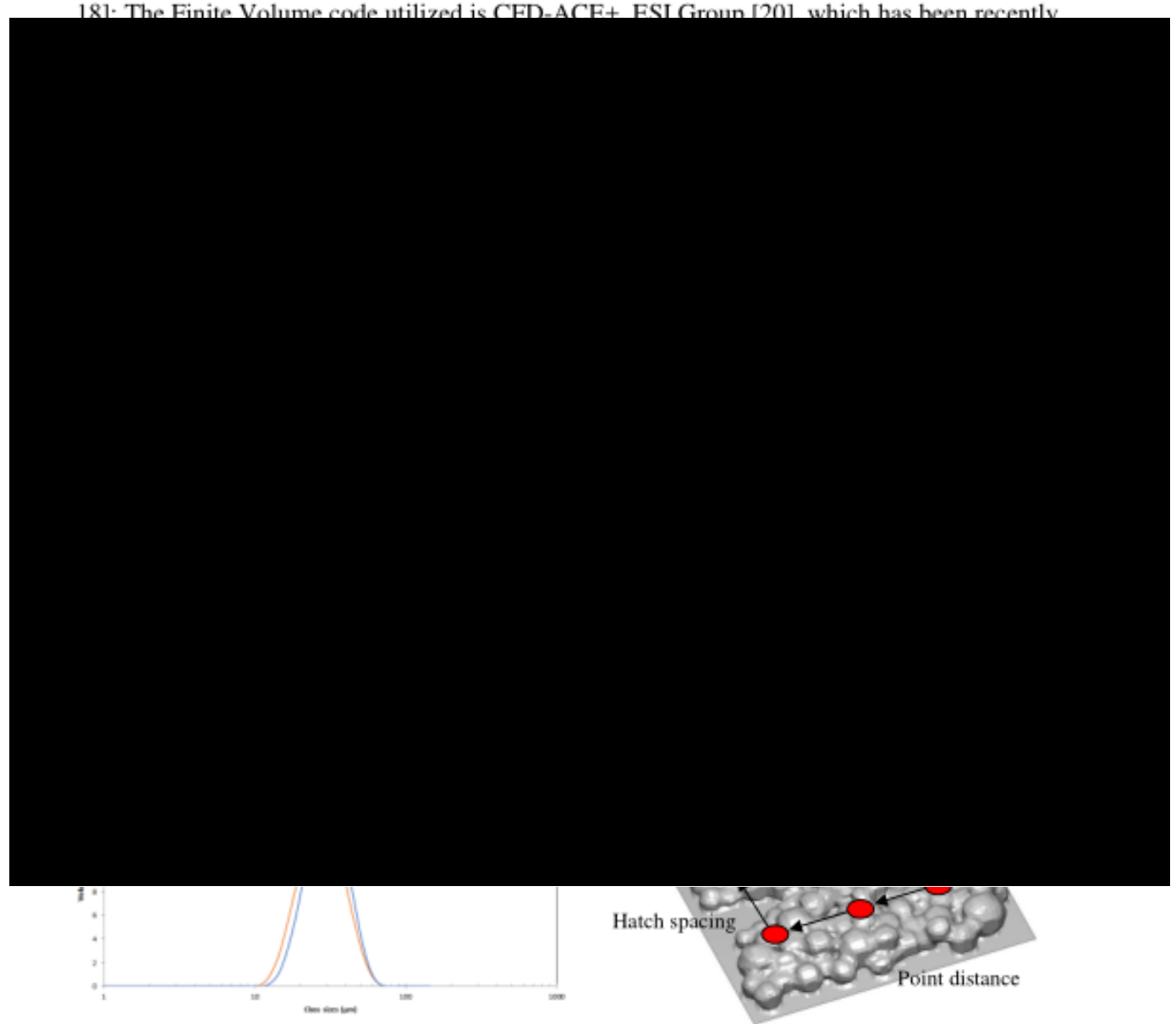

Figure 1: (a) LPW $316 \mathrm{~L}$ and Ti-6Al-4V powder size distributions and (b) the meandre laser track build strategy 
Experiment 1 (Single layer $316 \mathrm{~L}$ )

In experiment (E1) a single layer of $316 \mathrm{~L}$ was melted onto the base plate in a meander pattern using the point distance $(\mathrm{PD}=65 \mu \mathrm{m})$ and hatch spacing $(\mathrm{HS}=124 \mu \mathrm{m})$. Various exposure times and laser powers were used, and micrographs were taken from the top and at cross-sections through the laser tracks (Figure 2) for a laser power of $200 \mathrm{~W}$ and an exposure time of $150 \mu \mathrm{s}$. The measurements of the $316 \mathrm{~L}$ melt bead onto the base plate give typical widths of about $100 \mu \mathrm{m}$ ( $30 \mu \mathrm{m}$ wider than the laser beam) and heights of about $40-50 \mu \mathrm{m}$. There is typically a shallow (elliptical shaped) heat affected zone below the bead into the base plate (Figure 2 (b)). The bead cross section is etched and there is a clear difference between the larger grain structures in the underlying base plate, also made from $316 \mathrm{~L}$ steel and there is evidence of pitting in the heat

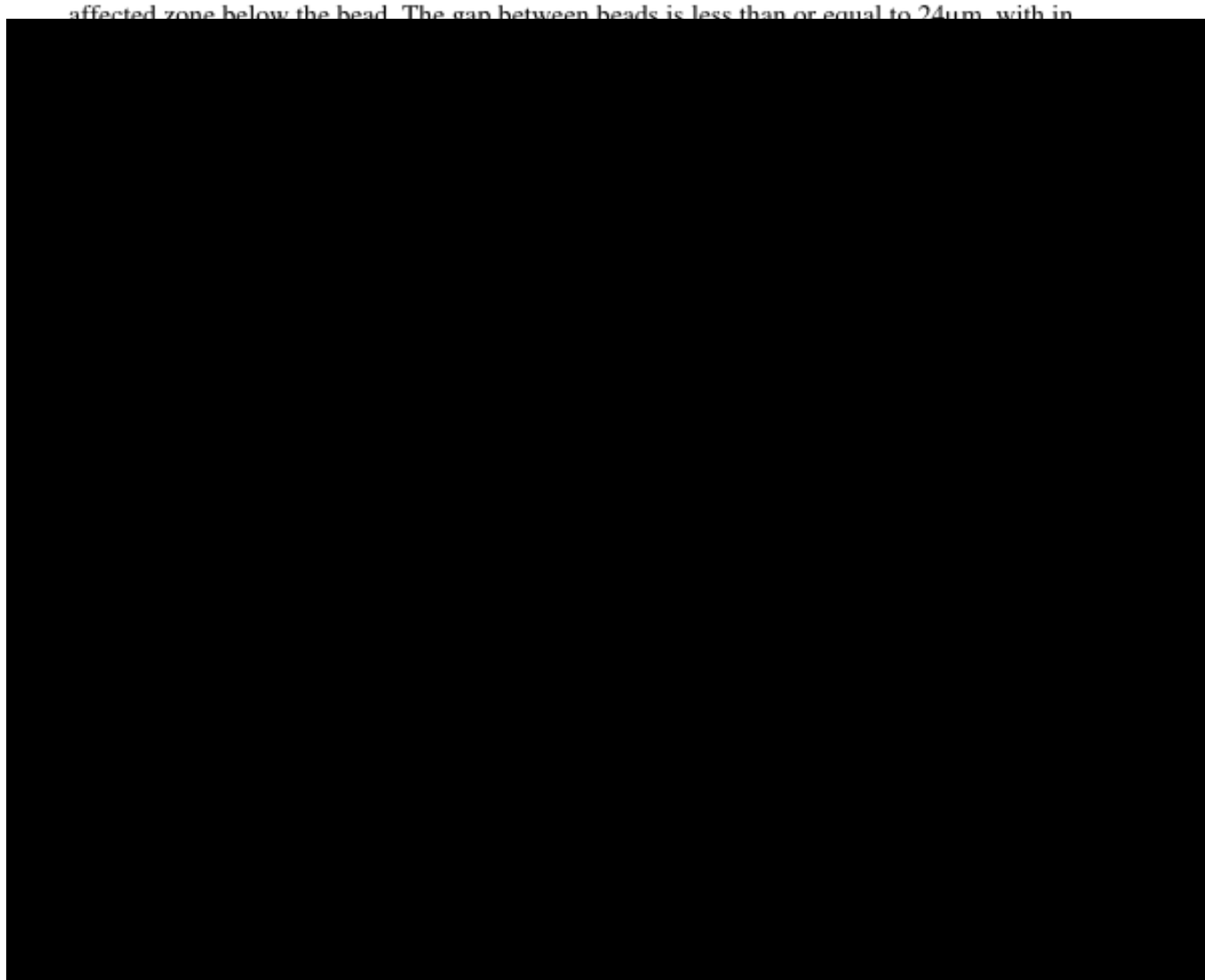

Rayleigh-Plateau instabilities. Measurements would indicate a laser track width of about $82 \mu \mathrm{m}$ which is only marginally higher than the laser diameter, however, it should be pointed out that this was done directly onto the powder, with less heat transmitted vertically it would be expected that the bead would have a higher dome than if melted onto the base plate, where it would run off closing the gaps between tracks slightly more. In normal builds using the same laser settings, 
relative densities have been measured in the $97-99 \%$ range with low porosity identified by micrographs. This would suggest that the re-melting during a multi-layer deposition works in much the same way as seen in experiment 2 , and any holes in the underlying layer are filled by subsequent melt liquid and smoothed by re-melting.

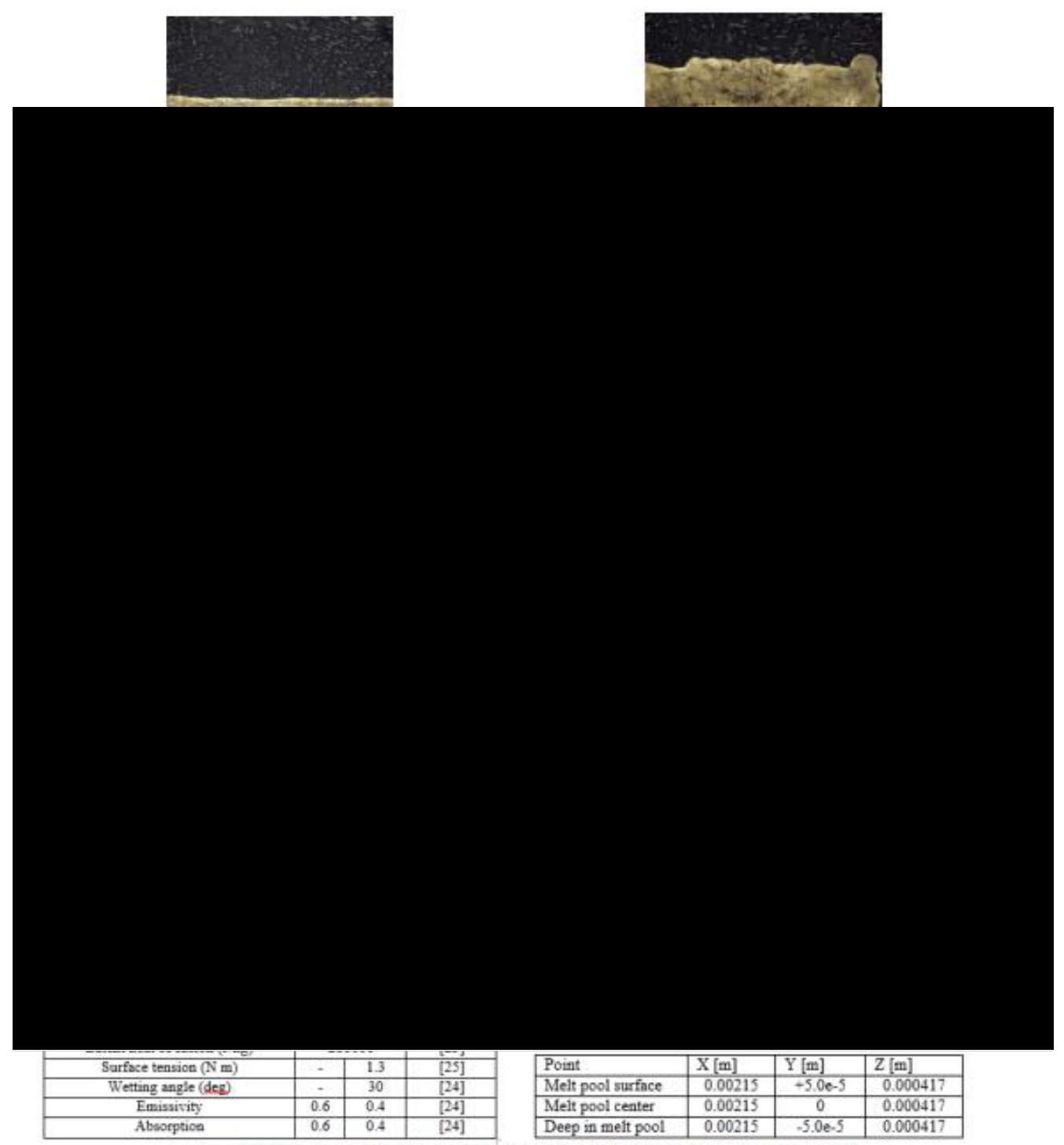

Table 2: T-Al4-V4 Properties Operation Condition and Probe co-ordinotes used for benchmark 


\section{Results \& Discussion}

Results presented here are those obtained for Ti-Al6-V4. Figure 1(b) shows the reference powder bed utilized for all calculations and the corresponding laser track. The particles representation is shown as resolved by the computational model. This particular specimen of the powder bed was chosen because it offers an interesting combination of smaller particles packed densely close to one another as well as some large particles that were pushed ahead of the coater causing some areas to be free of powder. Figure 5 shows a comparison of the melt pool shape as predicted by

comparing two codes that were developed separately at ESI Group and University of Swansea. The qualitative results are comparable showing similar melt pools sizes and capturing solidified surface irregularities. The cooling rate is in the order of $1 \mathrm{e} 6^{\circ} \mathrm{C} / \mathrm{s}$. 

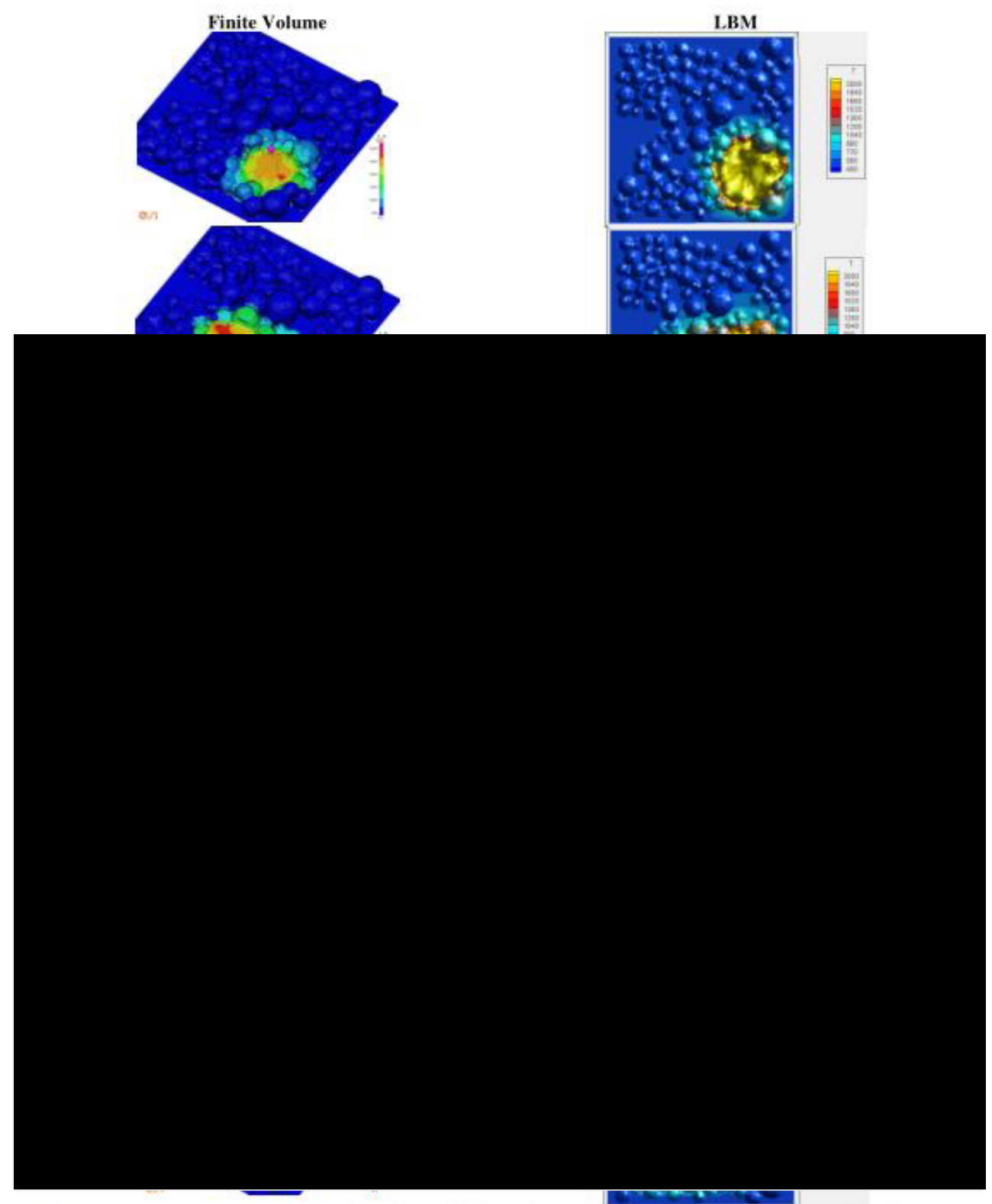

Figure 5: Top view comparison of melt pool evolution: Left finite volume results with full range temperature scale. Right LBM results with limited legend range showing more details of the particle meiting 
$1651-1662$.

3. Thermal and Mechanical Finite Element Modeling of Laser Forming from Metal and Ceramic Powders. K. Dai, L. Shaw. [ed.] Elsevier Ltd. 2004, Acta Materialia, Vols. 52: 69-80.

4. A Three Dimensional Finite Element Analysis of the Temperature Field During Laser Melting of Metal Powders in Additive Layer Manufacturing. I.A. Robert, C.J. Wang, R. Esterlein, M. Stanford, D.J. Mynors. s.1. : Elsevier Ltd., 2009, International Journal f Machine Tools \& Manufacture, Vols. 49: pp. 916-923. 


\section{Appendix 2 - Metallographic Preparation for Experiment A}

Each horizontal and vertical sample was mounted in a conductive thermosetting mounting resin Konductomet, supplied by Buehler. Each sample mount was mounted using the Buehler SimpliMet XPS1 compression mounting machine.

The grinding and polishing of the samples were performed using an AutoMet 300 Buehler grinder-polisher. The parameters used in the table below were used to obtain the optimum polishing results with the least amount of scratches, and were used to prepare each sample:

\begin{tabular}{|c|c|c|c|c|c|}
\hline $\begin{array}{c}\text { Abrasive Disk/ } \\
\text { Polishing cloth } \\
\text { used }\end{array}$ & $\begin{array}{l}\text { Lubricant/ Polishing } \\
\text { Suspension used }\end{array}$ & $\begin{array}{l}\text { Applied } \\
\text { Force }(\mathrm{N})\end{array}$ & $\begin{array}{l}\text { Disk } \\
\text { Speed } \\
\text { (RPM) }\end{array}$ & $\begin{array}{l}\text { Head } \\
\text { Speed } \\
\text { (RPM) }\end{array}$ & $\begin{array}{c}\text { Time } \\
\text { (minutes) }\end{array}$ \\
\hline $\begin{array}{c}\text { Silicon Carbide } \\
\text { Grinding Paper, } \\
600 \text { Grit }\end{array}$ & Water & 27 & 150 & 30 & 4 \\
\hline $\begin{array}{l}\text { Buehler Hercules } \\
\text { H Grinding Disk }\end{array}$ & $\begin{array}{c}\text { Buehler MetaDi } \\
\text { Supreme } 9 \mu \mathrm{m} \\
\text { Diamond Suspension }\end{array}$ & 27 & 150 & 30 & 2 \\
\hline Buehler Ultrapad & $\begin{array}{c}\text { Buehler MetaDi } \\
\text { Supreme } 9 \mu \mathrm{m} \\
\text { Diamond Suspension }\end{array}$ & 27 & 150 & 30 & 6 \\
\hline $\begin{array}{l}\text { Buehler TriDent } \\
\text { 3um Polishing } \\
\text { Cloth }\end{array}$ & $\begin{array}{c}\text { Buehler MetaDi } \\
\text { Supreme } 3 \mu \mathrm{m} \\
\text { Diamond Suspension }\end{array}$ & 13 & 150 & 30 & 6 \\
\hline
\end{tabular}

Between each grinding and polishing phase, the samples were washed first with water followed by ethanol. The samples were quickly dried under hot air after being washed with ethanol.

Each sample was etched with a solution of $100 \mathrm{~mL}$ ethanol, $100 \mathrm{~mL}$ hydrochloric acid at $48 \%$ concentration and $5 \mathrm{~g}$ of Copper(II) Chloride, commonly referred to as Kalling's Reagent No.2. The samples were immersed in the etchant for 20-90 seconds, washed clean with water and ethanol and dried under hot air. 


\section{Appendix 3 - Metallographic Preparation for Experiment $C$}

Each horizontal and vertical sample was mounted in a conductive thermosetting mounting resin Konductomet, supplied by Buehler. Each sample mount was mounted using the Buehler SimpliMet XPS1 compression mounting machine.

The grinding and polishing of the samples were performed using an AutoMet 300 Buehler grinder-polisher. The parameters used in the table below were used to obtain the optimum polishing results with the least amount of scratches, and were used to prepare each sample:

\begin{tabular}{|c|c|c|c|c|c|}
\hline $\begin{array}{l}\text { Abrasive Disk/ } \\
\text { Polishing cloth } \\
\text { used }\end{array}$ & $\begin{array}{l}\text { Lubricant/ Polishing } \\
\text { Suspension used }\end{array}$ & $\begin{array}{l}\text { Applied } \\
\text { Force }(\mathrm{N})\end{array}$ & $\begin{array}{l}\text { Disk Speed } \\
\text { (RPM) }\end{array}$ & $\begin{array}{l}\text { Head } \\
\text { Speed } \\
(\mathrm{RPM})\end{array}$ & Time (minutes) \\
\hline $\begin{array}{l}\text { Buehler Hercules } \\
\text { H Grinding Disk }\end{array}$ & $\begin{array}{c}\text { Buehler MetaDi Supreme } \\
6 \mu \mathrm{m} \text { Diamond } \\
\text { Suspension }\end{array}$ & 13 & 300 & 40 & $\begin{array}{l}\text { Until plane (usually } \\
20-30 \text { seconds) }\end{array}$ \\
\hline Buehler Ultrapad & $\begin{array}{c}\text { Buehler MetaDi Supreme } \\
9 \mu \mathrm{m} \text { Diamond } \\
\text { Suspension }\end{array}$ & 13 & 200 & 40 & 5 \\
\hline Buehler Ultrapad & $\begin{array}{c}\text { Buehler MetaDi Supreme } \\
6 \mu \mathrm{m} \text { Diamond } \\
\text { Suspension }\end{array}$ & 13 & 200 & 40 & 5 \\
\hline Buehler TriDent & $\begin{array}{c}\text { Buehler MetaDi Supreme } \\
3 \mu \mathrm{m} \text { Diamond } \\
\text { Suspension }\end{array}$ & 13 & 200 & 40 & 10 \\
\hline Buehler TriDent & $\begin{array}{c}\text { Buehler MetaDi Supreme } \\
1 \mu \mathrm{m} \text { Diamond } \\
\text { Suspension }\end{array}$ & 13 & 250 & 40 & 15 \\
\hline
\end{tabular}

Between each grinding and polishing phase, the samples were washed first with water followed by ethanol. The samples were quickly dried under hot air after being washed with ethanol.

Each mounted was etched using Beraha II colour etchant. The preparation and appropriate precautions for using Beraha II is listed in Appendix 4. The samples were immersed in the etchant for 60-90 seconds, washed clean with water and propanol and dried under hot air. 


\section{Appendix 4 - Beraha II Etchant Preperation}

\section{Materials}

Beraha II stock solution:

- $800 \mathrm{~mL}$ distilled water

- $400 \mathrm{~mL}$ hydrochloric acid $32 \%$

- $48 \mathrm{~g}$ ammonium hydrogen fluoride

Final etchant:

- $100 \mathrm{~mL}$ Beraha II stock solution

- $1 \mathrm{~g}$ potassium disulfite

Precautions: Wear goggles, gloves, vapour and dust respirator and synthetic apron on top of lab coat before handling materials. Prepare all solutions in plastic containers, such as polypropylene, as ammonium bifluoride can attack glass and metals. The apparatus used for mixing the solution should also be made of plastic. Handle all materials under an active fume hood.

Storage of stock solution: Store only in original receptacle, keep container tightly sealed. Storage class 8B, non-combustible corrosive liquid.

Disposal of stock solution: Must not be disposed together with regular waste. Do not allow product to reach sewage system.

Storage of final prepared etchant: Store only in original receptacle, keep container tightly sealed. Storage class 8B, non-combustible corrosive liquid. Keep in a cool, well-ventilated area.

Disposal of final prepared etchant: Exactly the same as that of the stock solution.

\section{Method}

To prepare the stock solution:

1. Add $400 \mathrm{~mL}$ of $32 \%$ hydrochloric acid to $800 \mathrm{~mL}$ of distilled water, slowly inside a fume cupboard.

2. Add $48 \mathrm{~g}$ of ammonium hydrogen fluoride. Allow to dissolve before moving on.

To prepare final etchant: 
1. Add $1 \mathrm{~g}$ of potassium disulfite to $100 \mathrm{~mL}$ of the stock solution, allowing it to dissolve before starting the etch. Solution can be stored and used within 1 to 2 hours. 


\section{Appendix 5 - Metallographic Preparation for Experiment E}

Any sample with successfully built lines was sawed in half using a Buehler IsoMet 4000

Precision Cutter and subsequently mounted in Metprep Conducto-Mount conductive mounting compound. This was done using a Buehler SimpliMet XP51 mounting system. The crucibles of the same set of parameters were mounted together for convenience. The mounted samples were then ground and polished according to the procedures listed in the table below.

\begin{tabular}{|c|c|c|c|c|c|}
\hline $\begin{array}{l}\text { Abrasive Disk/ } \\
\text { Polishing cloth } \\
\text { used }\end{array}$ & $\begin{array}{l}\text { Lubricant/ Polishing } \\
\text { Suspension used }\end{array}$ & $\begin{array}{l}\text { Applied } \\
\text { Force (N) }\end{array}$ & $\begin{array}{l}\text { Disk } \\
\text { Speed } \\
\text { (RPM) }\end{array}$ & $\begin{array}{l}\text { Head } \\
\text { Speed } \\
\text { (RPM) }\end{array}$ & $\begin{array}{c}\text { Time } \\
\text { (minutes) }\end{array}$ \\
\hline $\begin{array}{c}\text { Silicon Carbide } \\
\text { Grinding Paper, } \\
600 \text { Grit }\end{array}$ & Water & 27 & 300 & 40 & 5 \\
\hline $\begin{array}{c}\text { Silicon Carbide } \\
\text { Grinding Paper, } \\
1200 \text { Grit } \\
\end{array}$ & Water & 27 & 150 & 40 & 5 \\
\hline Buehler Ultrapad & $\begin{array}{c}\text { Buehler MetaDi } \\
\text { Supreme } 9 \mu \mathrm{m} \\
\text { Diamond Suspension }\end{array}$ & 27 & 150 & 30 & 10 \\
\hline $\begin{array}{l}\text { Buehler } \\
\text { ChemoMet }\end{array}$ & $\begin{array}{c}\text { Buehler MasterMet } \\
0.05 \mu \mathrm{m} \text { Colloidal } \\
\text { Silica }\end{array}$ & 22 & 150 & 30 & 10 \\
\hline
\end{tabular}

The polished samples were etched inside a fume cupboard using a batch of Kroll's reagent. The batch was made from the following $5 \mathrm{ml} \mathrm{HNO}^{3}, 10 \mathrm{ml} \mathrm{HF}$ at $48 \%$ concentration, and $85 \mathrm{ml}$ distilled water. Gloves and protective eyewear were worn whilst etching. Each specimen was swabbed with the reagent for $15-20$ seconds. 